\title{
EASTERN CARIBBEAN CURRENCY UNION: 2016 DISCUSSION ON COMMON POLICIES OF MEMBER COUNTRIES—PRESS RELEASE AND STAFF REPORT
}




\title{
INTERNATIONAL MONETARY FUND
}

IMF Country Report No. 16/333

\section{EASTERN CARIBBEAN CURRENCY UNION}

\section{DISCUSSION ON COMMON POLICIES OF MEMBER COUNTRIES-PRESS RELEASE AND STAFF REPORT}

October 2016

Under Article IV of the IMF's Articles of Agreement, the IMF holds bilateral discussions with members, usually every year. In the context of the 2016 Discussion on Common Policies of Member Countries, the following documents have been released and are included in this package:

- A Press Release summarizing the views of the Executive Board as expressed during its July 13, 2016 consideration of the staff report that concluded the 2016 Discussion on Common Policies of Member Countries.

- The Staff Report prepared by a staff team of the IMF for the Executive Board's consideration on July 13, 2016, following discussions that ended on April 29, 2016, with the officials of Member Countries of the Eastern Caribbean Currency Union on economic developments and policies. Based on information available at the time of these discussions, the staff report was completed on June 23, 2016.

- An Informational Annex prepared by the IMF staff.

The IMF's transparency policy allows for the deletion of market-sensitive information and premature disclosure of the authorities' policy intentions in published staff reports and other documents.

\author{
Copies of this report are available to the public from \\ International Monetary Fund • Publication Services \\ PO Box 92780 • Washington, D.C. 20090 \\ Telephone: (202) 623-7430 • Fax: (202) 623-7201 \\ E-mail: publications@imf.org Web: http://www.imf.org \\ Price: $\$ 18.00$ per printed copy
}

\section{International Monetary Fund Washington, D.C.}


Press Release No. 16/376

FOR IMMEDIATE RELEASE

August 11, 2016
International Monetary Fund

$70019^{\text {th }}$ Street, NW

Washington, D. C. 20431 USA

\section{IMF Executive Board Concludes 2016 Discussion on Common Policies of Member Countries of the Eastern Caribbean Currency Union}

On July 13, 2016, the Executive Board of the International Monetary Fund concluded the consideration of the 2016 discussion on the common policies of member countries of the Eastern Caribbean Currency Union.

The regional recovery is gaining ground, supported by continued low oil prices, strong tourism arrivals, and robust citizenship-by-investment receipts. Three failed banks have been resolved with no spillovers to the rest of the region and fiscal management has improved.

\section{Executive Board Assessment ${ }^{1}$}

Executive Directors welcomed the authorities' progress in addressing key challenges and the regional economic recovery, which is gradually gaining ground supported by strong external demand, low oil prices, and buoyant citizenship by investment receipts. Risks to the near term outlook are balanced, but growth in the ECCU continues to be hindered by weak competitiveness, banking sector fragilities, susceptibility to natural disasters, and large public debt. In this context, Directors encouraged the authorities to press ahead with sound macroeconomic policies and structural reforms to decisively address these issues and strengthen the conditions for robust long term growth.

Directors commended the decisive actions taken to strengthen the resilience of the banking system. In particular, they welcomed the passing of key banking legislation, and the successful resolution of three insolvent banks. With non-performing loans still elevated and credit continuing to decline, Directors stressed the importance of swift action to help banks clean up their balance sheets and resume lending. Specifically, they recommended quickly operationalizing the regional asset management company and moving ahead with regional foreclosure legislation. While Directors generally supported eliminating the minimum saving deposit rate, a few stressed the importance of accompanying this reform with measures to enhance the financial safety net and to avoid discouraging saving and reducing financial inclusion. Directors encouraged the authorities to promote consolidation within the regional indigenous banking system to strengthen its long run viability and help lower the risk of further

\footnotetext{
${ }^{1}$ At the conclusion of the discussion, the Managing Director, as Chairman of the Board, summarizes the views of Executive Directors, and this summary is transmitted to the country's authorities. An explanation of any qualifiers used in summings up can be found here: http://www.imf.org/external/np/sec/misc/qualifiers.htm.
}

Washington, D.C. 20431 • Telephone 202-623-7100 • Fax 202-623-6772 • www.imf.org 
withdrawal of correspondent banking relationships (CBRs) from the region. They agreed that efforts to strengthen frameworks for international tax cooperation and for anti-money laundering and combating the financing of terrorism should be complemented by continued engagement with international partners in order to mitigate the risk of further loss of CBRs. Directors supported further work in this area by the Fund to assist its members, and encouraged increased global cooperation to address this issue.

Directors welcomed the improvements in fiscal discipline, and emphasized that stronger action is needed to achieve the regional debt target of 60 percent of GDP by 2030. In particular, they recommended formulating detailed medium term fiscal adjustment plans and underpinning them with fiscal rules as well as continued enhancements to public finance management frameworks. Given the region's vulnerability to natural disasters, Directors generally underscored the importance of internalizing those costs in the fiscal policy frameworks. They also encouraged the authorities to develop a strong, regionally accepted set of principles and guidelines for citizenship by investment programs in order to enhance their sustainability.

Directors noted that the quasi currency board arrangement continues to serve the ECCU well by fostering price stability, and that international reserve levels exceed most of the Fund's reserve adequacy benchmarks. To improve international competitiveness, they encouraged the authorities to pursue structural reforms that reduce the costs of doing business. In this context, Directors commended the initiatives to reduce energy costs - particularly investments in renewable energy sources - and recommended policies to moderate labor costs, improve resilience to natural disasters, and deepen regional collaboration.

Directors recommended improving statistics and data provision in order to enhance economic analysis and better support policy making.

Directors agreed that the views they expressed today will form part of their discussions in the context of the Article IV consultations with individual ECCU members that will take place until the next Board discussion of ECCU common policies. 
ECCU: Selected Economic and Financial Indicators, 2013-21 1/

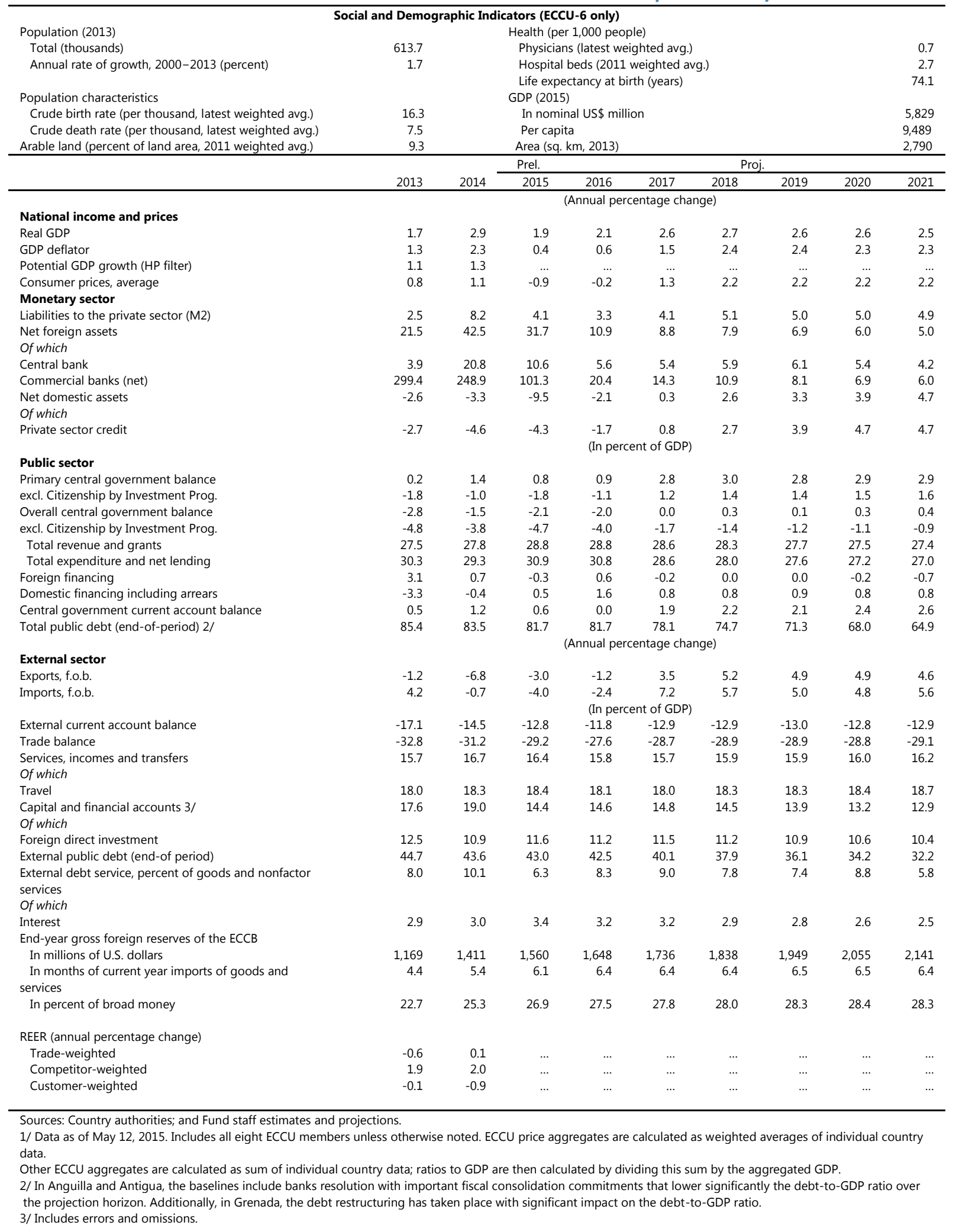

CInternational Monetary Fund. Not for Redistribution 


\section{INTERNATIONAL MONETARY FUND}

\section{EASTERN CARIBBEAN CURRENCY UNION}

June 23, 2016

\section{STAFF REPORT FOR THE 2016 DISCUSSION ON COMMON} POLICIES OF MEMBER COUNTRIES

Under Article IV of the IMF's Articles of Agreement, the IMF holds bilateral discussions with members, usually every year. In the context of the 2016 ECCU Common Policies Consultation, the mission held policy discussions with the Governor of the ECCB, national authorities in all eight member jurisdictions, financial system regulators, representatives of commercial banks, and senior officials of CDB.

\section{KEY ISSUES}

The regional recovery is gaining ground, supported by continued low oil prices, the return to pre-2007 levels of tourism arrivals, and buoyant citizenship-by-investment receipts. Three failed banks have been resolved with no spillovers to the rest of the region and authorities have demonstrated improved fiscal management. Risks in the short run appear to be balanced but the region still faces many vulnerabilities that jeopardize the medium-term outlook. This year's discussions took stock of the progress made and the policies needed to address key vulnerabilities related to the weak banking system, high debt, susceptibility to natural disasters, and competitiveness.

\section{Main Policy Recommendations:}

Banking system: Operationalize the regional asset management company; increase capital of undercapitalized indigenous banks; strengthen supervision by implementing IMF TA recommendations; and promote consolidation of the system.

Monetary policy: Eliminate the minimum saving deposit rate.

Fiscal policy: Implement medium-term fiscal adjustment programs consistent with achieving the debt target; incorporate expected costs of natural disasters in the design of fiscal policy; strengthen the fiscal framework, including with fiscal rules; improve management of citizenship programs.

Structural reforms: Increase competitiveness and potential growth by: lowering the cost of energy; improving resilience to natural disasters; reducing unit labor costs; and broadening regional collaboration.

Statistics: Improve BOP, Government Finance and Public Sector Debt statistics, and labor market statistics to allow adequate surveillance and policy analysis. 
Approved By

Krishna Srinivasan and Bob Traa
Mission Team: T. Alleyne (Head), A. Myrvoda, and G. Salinas (all WHD), J. Reynaud (LWOP), and M. Moore (MCM). The mission held policy discussions with authorities in all eight ECCU jurisdictions, including Prime Ministers Anthony (St. Lucia), Browne (Antigua and Barbuda), Gonsalves (St. Vincent and The Grenadines), Skerrit (Dominica), and Governor Antoine (ECCB). The mission also met with President Smith (CDB), financial system regulators, representatives of commercial banks, and senior officials of the OECS Commission, Contributors: ECCU team (T. Alleyne, A. Myrvoda, J. Reynaud, G. Salinas); S. Acevedo, K. Beaton, D. Cortez, A. El Ashram, A. Guerson, R. James, M. Li, J. Lafeuillee, M. Vargas, and H. Yun (all Caribbean I Division); M. Moore and M. Souto (both MCM); M. Smith (CARTAC); and S. Thompson (ECCB). J. Villacorte and R. Fonseca prepared the document and provided administrative assistance.

Mission Dates: March 9-18 and April 25-28, 2016

\section{CONTENTS}

A. ECCU Banking Sector: From Resolution to Restructuring

B. The Task of Monetary Policy: Unwinding the Interest Rate Floor, Excess Liquidity, and the

Credit Crunch

C. Global Withdrawal of Correspondent Banking Relationships (CBRs): A Need for Close Monitoring

D. Strengthening Fiscal Policy in the context of high debt, low fiscal buffers, and increasing natural disasters

E. Structural Reforms: A Menu of Options to Increase Competitiveness

STAFF APPRAISAL

\section{BOXES}

1. Risk Assessment Matrix ___ $\underline{7}$

2. Explaining High Unemployment in the ECCU__ 20

\section{FIGURES}

1. Real Sector Developments 
2. Tourism Developments __ $\underline{24}$

3. Monetary Developments___ $\underline{25}$

4. Financial Soundness Indicators__ $\underline{26}$

5. Doing Business Indicators___ 27

\section{TABLES}

1. Selected Economic and Financial Indicators, 2009-21_ 28

2. Selected Economic Indicators by Country, 2009-21__ 29

3. Selected Central Government Fiscal Indicators by Country, 2009-21_

4. Selected Public Sector Debt indicators by Country, 2009-21_

5. Monetary Survey, 2009-18__ $\underline{32}$

6. Summary Balance of Payments, 2009-21__

7. Recent Fund Staff Regional Consultation Recommendations___ 34

\section{ANNEXES}

I. Citizenship-by-Investment Programs in ECCU

II. ECCU Risks to Financial Stability $\underline{40}$

III. Spillovers from Trinidad and Tobago to ECCU ___ 43

IV. Safeguarding Financial Stability in ECCU___ $\underline{46}$

V. Non-Performing Loans in the ECCU: Determinants and Macroeconomic Impact __ $\underline{50}$

VI. Impact of the Withdrawal of Correspondent Banking Relationships (CBRs) on the ECCU __ $\underline{63}$

VII. Public Financial Management in the ECCU: An Examination of PEFA Results ___ $\underline{69}$

VIII. Assessing Government Self-Insurance Needs against Natural Disasters: An Application to the ECCU — $\underline{71}$

IX. Explaining High Unemployment in the ECCU ___ $\underline{86}$

X. ECCU Energy: Macro-Related Challenges___ 91

XI. United Kingdom Overseas Territories-Anguilla and Montserrat___ $\underline{96}$

XII. External Assessment in the ECCU___ 101 


\section{RECENT ECONOMIC DEVELOPMENTS}

1. After a robust pick up in 2014, regional growth moderated in 2015 , largely reflecting slower growth in tourist arrivals. ECCU regional growth decelerated from 2.9 percent $y / y$ in 2014 to an estimated 1.9 percent in 2015. Stay-over tourist arrivals decelerated from 6 percent y/y growth in 2014 to about 2.4 percent in 2015, mainly because of a sharp decline in arrivals from Canada, which experienced decelerated growth and a depreciation of its currency against the EC dollar. Inflation in the region turned negative due to declining commodity and subdued food prices.
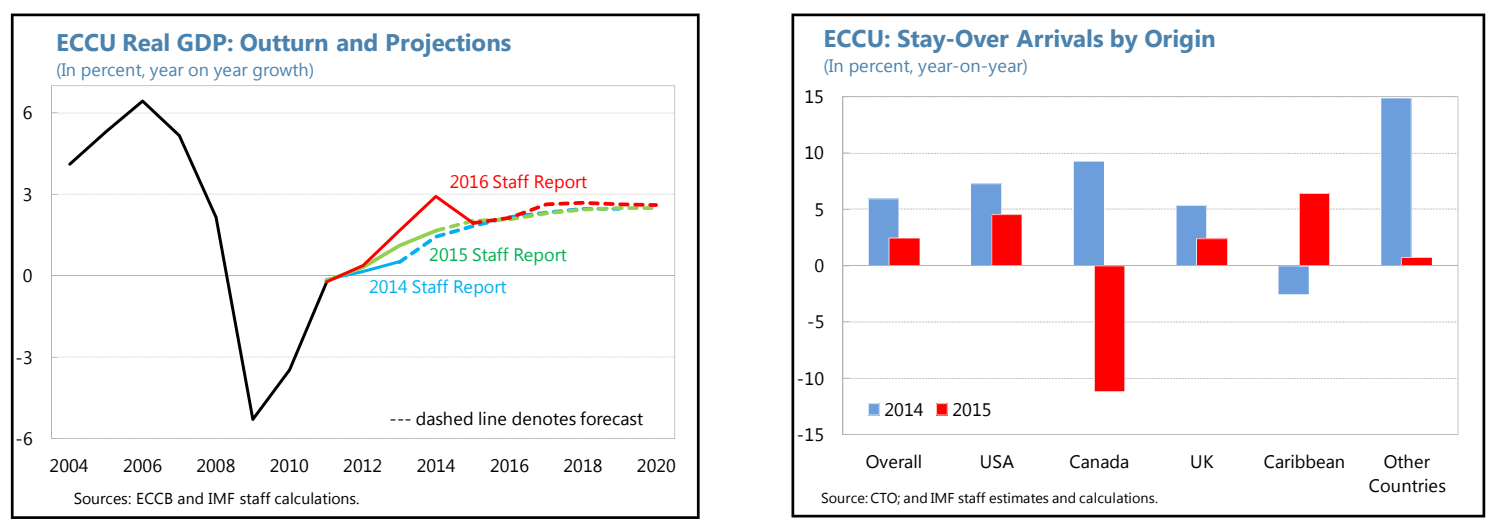

2. Overall fiscal performance improved in 2015. The regional public debt continued to decline in 2015, reaching 81.7 percent of GDP, down from the peak of 86 percent in 2012. The headline primary surplus narrowed from 1.4 percent of GDP in 2014 to an estimated 0.8 percent, but this was largely on account of bank resolution costs in Antigua and Barbuda. All other jurisdictions, except St. Kitts and Nevis and Anguilla, recorded improvements in their primary

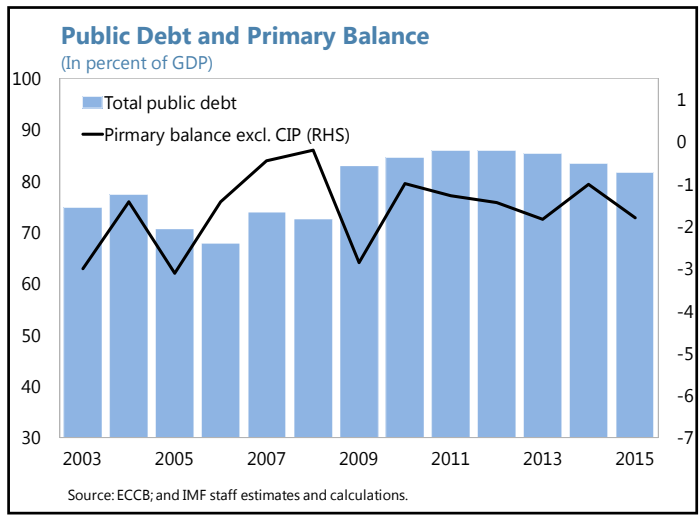
balances.

\section{Most governments continued to rely on Citizenship-by-Investment Programs (CIPs)} inflows to fund their budgets in 2015. Nevertheless, the underlying primary balance (i.e., excluding one-off items and CIP) improved from negative 1.5 percent of GDP in 2014 to an estimated negative 0.2 percent in $2015^{1}$. CIP inflows were highest in St. Kitts and Nevis, the region's longest standing program, with revenues to the public sector at 17.4 percent of GDP. Inflows reached 7.9 percent of GDP in 2015 in Antigua and Barbuda and 3.6 percent in Dominica.

${ }^{1}$ Excludes Anguilla and Montserrat. 
St. Lucia launched its program in early 2016, leaving only St. Vincent and the Grenadines without a CIP among the six independent ECCU countries (Annex 1).

\section{External sector performance} improved in 2015, owing mainly to expanding tourism and falling commodity prices. The regional current account deficit is estimated to have narrowed to 12.8 percent of GDP from 14.5 percent in 2014. ${ }^{2}$ Over 80 percent of the current account deficit was financed through FDI inflows, which continue to recover but, at 11.6 percent of GDP, remain well below the peak of 22 percent of GDP in 2007. Meanwhile, gross international reserves rose to an estimated 6 months of imports of goods and services in 2015, supported by strong CIP inflows.

5. Reforms to external sector statistics, using BPM6 and updated surveys, point to much smaller current account deficits for ECCU members. Early this year, the ECCB released preliminary external sector statistics for 2014. These data, which are in the process of verification and hence are not yet reflected in Fund tables, show current account deficits about 10 percentage points of GDP lower than BPM5 data. In addition to updated estimates of visitor expenditure, the new estimates covered a substantially larger sample of respondents, including offshore universities.
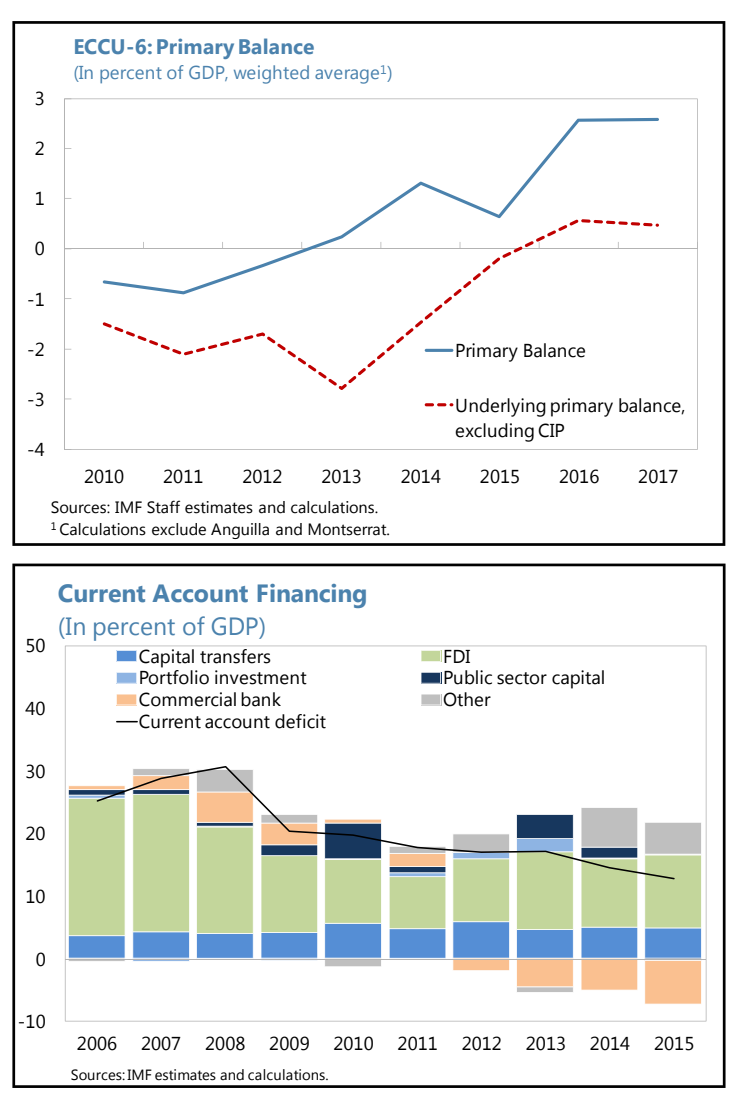

\begin{tabular}{|c|c|c|c|}
\hline \multicolumn{4}{|c|}{$\begin{array}{l}\text { Preliminary Current Account Data } \text { Revisions }^{1} \\
\text { (In percent of GDP, 2014) }\end{array}$} \\
\hline & BPM5 & BPM6 & Change \\
\hline \multicolumn{4}{|l|}{ Current Account } \\
\hline ECCU & -14.2 & -4.5 & 10 \\
\hline Anguila & -17.3 & -9.0 & 8 \\
\hline Antigua and Barbuda & -14.5 & -1.7 & 13 \\
\hline Dominica & -13.1 & -6.8 & 6 \\
\hline Grenada & -18.4 & -7.8 & 11 \\
\hline Montserrat & -44.4 & -18.2 & 26 \\
\hline St. Kitts and Nevis & -6.3 & 3.0 & 9 \\
\hline St. Lucia & -6.7 & 7.2 & 14 \\
\hline St. Vincent & -29.6 & -24.6 & 5 \\
\hline \multicolumn{4}{|c|}{ Sources: ECCB; and IMF staff estimates and calculations. } \\
\hline
\end{tabular}

\section{The authorities took important steps to enhance the resilience of the banking} sector, strengthening the bank resolution framework and resolving three insolvent banks (Paragraph 9). However, weak asset quality, low profitability, and insufficient capital in some banks continue to put a strain on financial stability (Annex 2). Despite some progress, the nonperforming loans ratio remains elevated, at 17 percent of total loans. Efforts by banks to clean up

\footnotetext{
2 Estimates based on BPM5 methodology.
} 
their balance sheets are often constrained by debtor-friendly foreclosure regulations, while low profitability restricts banks' ability to raise NPL provisioning from an inadequate 44 percent of non-performing loans. In this environment, banks have been reluctant to expand credit to the private sector, which fell by 4.2 percent $y / y$ in February 2016-the third consecutive year of decline. In contrast, bank deposits continue to expand. Banks have built up record amounts of excess liquidity, most of

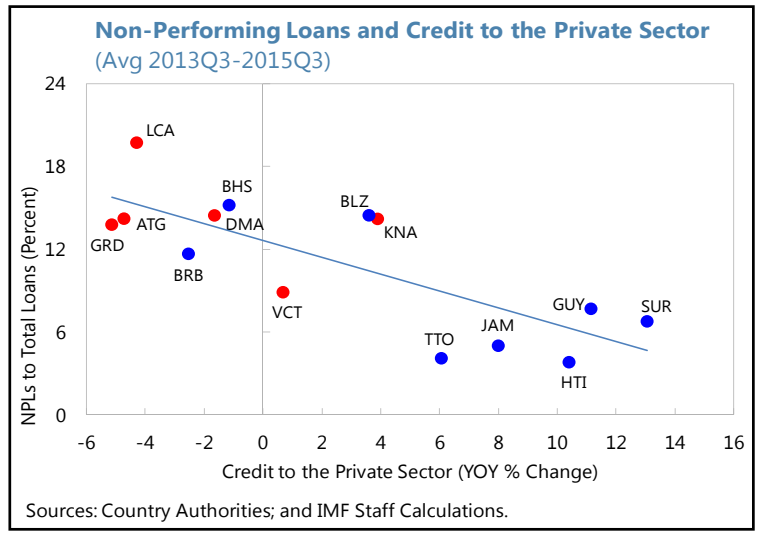
which is being held as reserves at the central bank. Many banks are also actively discouraging savings deposits, including turning away new such deposits.

\section{OUTLOOK AND RISKS}

7. ECCU growth is projected to converge to its long-term average of about $\mathbf{2 . 5}$ percent over the medium-term. Favorable economic conditions in the United States and the United Kingdom will continue to support the recovery in tourism, and growth in Canada is expected to regain momentum by 2017 as commodity prices are set to recover gradually. Greater passthrough of lower international oil prices to consumers in 2016 is expected to stimulate retail sales and boost domestic demand without generating any inflation pressures. The external current account deficit is projected to narrow to about 11.8 percent of GDP (using the BPM5 series), its smallest value since the late 1990s, mainly reflecting a lower oil import bill and further increases in tourist arrivals. In this context, import coverage of gross international reserves is projected to increase from 5.4 months in 2014 to 6.4 months in 2017.

8. Overall, risks to the baseline are balanced. (Box 1) An important downside risk relates to the withdrawal of correspondent banking relationships by international banks which could have serious negative implications for the business of indigenous banks and for the economy. Governments could face significant cash flow challenges given their large debts and debt servicing costs, especially if CIP inflows were to dry up. On the other hand, CIP flows could turn out to be much larger than projected, contributing to an easing of fiscal financing constraints and stronger growth via contributions to the construction and tourism sectors. Similarly, the decrease in commodity prices could support more income growth than assumed in the baseline, although the negative impact on key trading partners Trinidad \& Tobago and Canada could dampen some of the benefit (Annex 3). Finally, large infrastructure (international airport in St. Vincent and the Grenadines) and foreign direct investment projects (large hotel projects are under consideration in Antigua and Barbuda, Grenada, and St. Vincent and the Grenadines) could significantly boost economic growth beyond current estimates. 


\begin{tabular}{|c|c|c|c|}
\hline \multicolumn{4}{|c|}{ Box 1. ECCU: Risk Assessment Matrix (RAM) ${ }^{1}$} \\
\hline Nature/Source of Main Risks & $\begin{array}{l}\text { Relative } \\
\text { Likelihood }\end{array}$ & Expected Impact & Recommended Policy Response \\
\hline \multirow{3}{*}{$\begin{array}{l}\text { Persistent banking sector } \\
\text { weaknesses. ECCU banks } \\
\text { continue to report low } \\
\text { profitability, limited } \\
\text { capitalization, and high levels } \\
\text { of NPLs. }\end{array}$} & \multirow[t]{3}{*}{ High } & Medium & \multirow{3}{*}{$\begin{array}{l}\text { Require banks to increase capital; } \\
\text { operationalize the ECAMC; promote } \\
\text { bank consolidation. }\end{array}$} \\
\hline & & $\begin{array}{l}\text { Continued negative credit growth } \\
\text { and renascent risk of banking } \\
\text { system instability. }\end{array}$ & \\
\hline & & & \\
\hline \multirow[b]{2}{*}{$\begin{array}{l}\text { Reduced financial services } \\
\text { by global/regional banks } \\
\text { ("de-risking"). Further loss of } \\
\text { correspondent banking } \\
\text { services significantly curtails } \\
\text { cross-border payments, trade } \\
\text { finance, and remittances in } \\
\text { small economies. }\end{array}$} & \multirow[t]{2}{*}{ Medium } & Medium to High & \multirow[b]{2}{*}{$\begin{array}{l}\text { Continue to strengthen AML/CFT and } \\
\text { international tax cooperation } \\
\text { frameworks; promote bank } \\
\text { consolidation. Affected banks should } \\
\text { explore possibility of paying larger } \\
\text { unaffected banks a fee to carry out } \\
\text { cross border transactions on their } \\
\text { behalf. }\end{array}$} \\
\hline & & $\begin{array}{l}\text { A loss of correspondent banking } \\
\text { relationships would disrupt the } \\
\text { cross-border payments system, } \\
\text { impeding proper execution of } \\
\text { cross-border transactions over } \\
\text { short-term. }\end{array}$ & \\
\hline \multirow{2}{*}{$\begin{array}{l}\text { Persistently lower energy } \\
\text { prices, triggered by supply } \\
\text { factors reversing only } \\
\text { gradually. }\end{array}$} & \multirow[t]{2}{*}{ High } & Low & \multirow[t]{2}{*}{ Diversify export and tourism markets. } \\
\hline & & $\begin{array}{l}\text { Overall positive effect for the } \\
\text { region but strong impact could be } \\
\text { dampened given financial sector, } \\
\text { tourism, and trade linkages to } \\
\text { Canada and Trinidad and Tobago. }\end{array}$ & \\
\hline \multirow{2}{*}{$\begin{array}{l}\text { Natural disasters. Hurricanes } \\
\text { and floods causing } \\
\text { widespread damage, } \\
\text { destruction of physical capital, } \\
\text { and reduction in tourist } \\
\text { arrivals. }\end{array}$} & \multirow[t]{2}{*}{ Medium } & Medium to High & \multirow{2}{*}{$\begin{array}{l}\text { Explicitly account for natural disaster in } \\
\text { macroeconomic policy programming. } \\
\text { Build fiscal buffers, including using CIP } \\
\text { revenues. }\end{array}$} \\
\hline & & $\begin{array}{l}\text { Negative impact on growth; } \\
\text { increased debt. }\end{array}$ & \\
\hline \multirow{2}{*}{$\begin{array}{l}\text { Opening of U.S.-Cuba } \\
\text { tourism, following the } \\
\text { regularization of diplomatic } \\
\text { relations between the two } \\
\text { countries. }\end{array}$} & \multirow[t]{2}{*}{ High } & Low & \multirow{2}{*}{$\begin{array}{l}\text { Adopt a comprehensive approach to } \\
\text { raise competitiveness; build regional } \\
\text { synergy against potential negative } \\
\text { shocks. }\end{array}$} \\
\hline & & $\begin{array}{l}\text { The effect on the ECCU is unclear. } \\
\text { Research so far suggests ECCU } \\
\text { niche could be preserved and } \\
\text { downside risks are expected to be } \\
\text { manageable. }\end{array}$ & \\
\hline \multirow{2}{*}{$\begin{array}{l}\text { Fall/Increase in citizenship- } \\
\text { by-investment (CIP) } \\
\text { receipts. }\end{array}$} & \multirow[t]{2}{*}{ Medium } & High & \multirow{2}{*}{$\begin{array}{l}\text { Use CBI revenues to reduce debt, build } \\
\text { buffers, and finance large } \\
\text { infrastructure projects. Strengthen due } \\
\text { diligence and oversight to reduce } \\
\text { financial integrity and international } \\
\text { security risks. }\end{array}$} \\
\hline & & $\begin{array}{l}\text { A drop in CBI revenues would add } \\
\text { to government financing pressures } \\
\text { for some ECCU members. } \\
\text { Conversely, an increase in CBI } \\
\text { revenues would ease financing } \\
\text { pressures. }\end{array}$ & \\
\hline \multirow[t]{2}{*}{ Spread of Zika virus. } & \multirow[t]{2}{*}{ Medium } & Medium & \multirow{2}{*}{$\begin{array}{l}\text { Enhance eradication and protection } \\
\text { actions; implement public education } \\
\text { campaign. }\end{array}$} \\
\hline & & $\begin{array}{l}\text { Very few cases detected thus far. } \\
\text { Further spread of Zika virus could } \\
\text { adversely affect growth, including } \\
\text { by reducing tourist arrivals }\end{array}$ & \\
\hline \multicolumn{4}{|c|}{$\begin{array}{l}\text { 1/ The RAM shows events that could materially alter the baseline path (the scenario most likely to materialize in the view of IMF staff). The } \\
\text { relative likelihood is the staff's subjective assessment of the risks surrounding the baseline ("low" is meant to indicate a probability below } 10 \\
\text { percent, "medium" a probability between } 10 \text { and } 30 \text { percent, and "high" a probability between } 30 \text { and } 50 \text { percent). The RAM reflects staff views } \\
\text { on the source of risks and overall level of concern as of the time of discussions with the authorities. Non-mutually exclusive risks may interact } \\
\text { and materialize jointly. }\end{array}$} \\
\hline
\end{tabular}




\section{POLICY DISCUSSIONS}

Macro-financial issues as well as the conduct of monetary policy under the ECCU's quasi currency board arrangement featured prominently in discussions with the authorities. Topics included the necessary steps-beyond resolving the intervened banks - to further strengthen the banking system to facilitate a resumption of credit; the efficacy of eliminating the minimum saving deposit rate; and the growing threat of the withdrawal of correspondent banking relationships (CBRs) by international banks. Another key topic was how to optimize the use of citizenship program inflows and better manage risks associated with a sudden stop. Discussions also focused on policies to improve resilience to natural disasters and regional approaches to increasing competitiveness and potential growth.

\section{A. ECCU Banking Sector: From Resolution to Restructuring}

Recent legislative reforms and the successful resolution of insolvent banks need to be followed quickly by additional actions to further improve the health and stability of the banking system, including operationalizing the regional asset management company, strengthening supervisory processes, increasing capital of the indigenous banks and promoting consolidation of the system.

\section{Background}

\section{The authorities have taken decisive action in the past year to address banking system fragilities (Annex 4).}

- All jurisdictions have approved the regional legislative reforms, which are now in effect. The new Banking Act significantly improves the bank resolution framework; increases ECCB's regulatory powers, including to license commercial banks and approve and remove bank management and board members; raises minimum bank capitalization; and prioritizes small depositors' interests in the event of a liquidation. The Eastern Caribbean Asset Management Company (ECAMC) Act provides the legal authority for the ECAMC, which will have comprehensive powers to expedite the collection of NPLs or their collateral.

- The resolution of the insolvent bank in Antigua and Barbuda was completed in November 2015. The good assets of the former ABI Bank along with deposits up to $E C \$ 500,000$ were acquired by the Eastern Caribbean Amalgamated Bank via a purchase and assumption (P\&A) agreement. Deposits above EC $\$ 500,000$ were transferred to a Depositor Trust Fund backed by a EC $\$ 157$ million (4.5 percent of GDP) government bond. These depositors will be paid off over 10 years as the bond is amortized. The bad assets and remaining liabilities were transferred to a receivership.

- After many delays, the two insolvent banks in Anguilla were resolved in late April 2016. The U.K. authorities required a number of clarifications on the proposed resolution strategy and reassurances on the government's medium-term economic and fiscal plans. The resolution 
structure was similar that used in Antigua and Barbuda, except that in Anguilla, deposits up to a EC $\$ 2.8$ million threshold were transferred to a new government-owned bridge bank along with the good assets of the intervened banks and some non-performing loans. Deposits above EC $\$ 2.8$ million, including those of the Social Security system, amounting to EC $\$ 265$ million (29.9 percent of GDP), were transferred to Deposit Protection Trusts backed by the issue of new bonds, which will be amortized over 10-25 years. Non-performing assets as well as placements by offshore subsidiaries in the parent banks were put in receivership.

10. However, more reforms are needed to durably strengthen the banking system. The ECAMC remains to be operationalized. Asset quality reviews (AQRs) and viability assessments (dynamic modeling, DM) have been completed that identified capital shortfalls in some indigenous banks and an overbanked system. Also, Fund TA has identified weaknesses in the bank supervision process.

11. The $\mathbf{2 0 1 6}$ Safeguards Assessment for the ECCB concluded earlier this year. The assessment concluded that the ECCB has maintained sound external audit arrangements and transparent financial reporting practices. It also recognized that the governance arrangements at the ECCB provide for independent oversight, while recommending that its Board Audit Committee maintain access to an external financial expert to enhance its oversight. On the other hand, the assessment concluded that the internal audit function falls short of international standards, as the extensive involvement of the Internal Audit Department (IAD) in the bank's operations compromises its independence.

\section{Staff Policy Recommendations}

12. The authorities should move quickly to operationalize the ECAMC, which is expected to catalyze the recovery of bad assets transferred to receiverships. The ECCB is targeting June 2016 for the opening of the ECAMC and is now confirming the new directors; obtaining capital contributions from ECCU member jurisdictions; and hiring staff. To help get the ECAMC up and running, the Fund will provide technical assistance, funded by the CDB. Beyond taking over the receiverships in Antigua and Anguilla, the ECAMC is expected to play a key role in cleaning up the balance sheets of the other indigenous banks by purchasing their NPLs. There will also be a need to reform alien land holding laws in some jurisdictions (e.g., Anguilla) in order to facilitate the ECAMC's sale of the assets.

13. The ECCB should order corrective actions, including capital calls, as soon as possible from undercapitalized-but-viable banks identified in the asset quality reviews. Banks will then have to submit sound capitalization plans to the ECCB and follow through over an adequate time-horizon. Banks that do not comply with the capitalization plans may have to merge with or be acquired by other banks. Non-viable banks would need to exit the market in an orderly fashion. In this connection, staff and the ECCB agreed that the reform process should aim to consolidate the number of indigenous banks. Staff is supporting the ECCB in its work to look at various merger options that would reduce concentration and liquidity risk, while improving 
profitability prospects. The ECCB has also commissioned technical assistance from the World Bank to study optimal indigenous bank consolidation strategies.

14. The authorities need to implement recent technical assistance aimed at enhancing supervisory processes. The ECCB has received significant Fund technical assistance aimed at strengthening its supervisory capacity, particularly with respect to loan classification, collateral valuation, on- and off-site inspections, and implementation of Basel II. Regarding the Safeguards Assessment, staff recommended that the ECCB reassign the internal audit department's operational duties to other departments to address conflict of interest issues.

\section{Authorities' views}

\section{The ECCB and country authorities expressed their appreciation for the Fund's} helpful role in successfully resolving the insolvent banks. The ECCB agreed with the staff's recommendations and noted that a new comprehensive program of Fund TA has been agreed and is being implemented. Country authorities and bank CEOs supported the objective to consolidate the banking system. Some authorities warned that proposals should not entail any significant use of fiscal resources. Some bank CEOs thought that convincing bank shareholders on the efficacy of consolidation could be difficult. The authorities broadly agreed with the Safeguards Assessment's findings and recommendations and expressed a commitment to implementing them.

\section{B. The Task of Monetary Policy: Unwinding the Interest Rate Floor, Excess Liquidity, and the Credit Crunch}

The ECCB continues to follow policies consistent with maintaining the stability of the currency. However, it should eliminate the minimum saving deposit rate, which has undercut banks' ability to clean up their balance sheets and contributed to the protracted decline in credit.

\section{Background}

\section{The ECCB has effectively maintained the stability of the EC dollar in the context of} the quasi-currency board arrangement. The ratio of international reserves to money base was 96 percent at end-2015, compared with the statutory mandate of 60 percent. ${ }^{3}$ The central bank has achieved this without the active use of monetary policy instruments. Its main monetary policy tool, the discount rate, has remained at 6.5 percent since July 2003. The minimum saving deposit rate (MSR), which was introduced in 1985 at 4 percent, has been changed only twice, most recently in May 2015, when it was reduced from 3 percent to 2 percent. In both cases the decision was taken to address low profitability in the banking sector.

\footnotetext{
3 By comparison, The Bahamas and Barbados, the two other countries with long-standing fixed exchange rate pegs, have reserve coverage ratios of 73 percent and 46 percent, respectively.
} 


\section{While the MSR was established to provide an investment vehicle for small} depositors, its negative effects now appear to outweigh its benefits. Even with the decline last year, the MSR continues to distort banking conditions. By preventing a decline in deposit rates in the current context of excess liquidity, the MSR undercuts banks' profitability. The MSR also has impeded a reduction in lending rates, thereby reinforcing weak loan demand and excess liquidity conditions in the banks. The long history of the existence of the MSR has also likely reduced incentives for regional capital market development as satisfaction with a risk-free guaranteed rate of return through saving deposits has diminished the appetite and the incentives to develop other financial instruments.

\section{Staff Recommendations}

18. Staff recommended the immediate elimination of the minimum saving deposit rate or restricting its applicability to small saving deposits. Staff analysis suggests that reducing the saving deposit floor by 1 percentage point (ppt) would lower deposit rates by 0.7 ppts, while lending rates would decline by about 0.4 ppts. ${ }^{4}$ Staff analysis also suggests that a positive shock to profitability (or alternatively a negative shock to the expense-to-income ratio), potentially triggered by a lower saving deposit floor, would reduce NPLs, which in turn would have a positive effect on credit and growth. Staff surveyed the regional banking community and found that the decline in the MSR in May 2015 was transmitted into lower prime mortgage rates and, to a lesser degree, into prime consumer lending rates. ${ }^{5}$ Interviewed banks reported passing through to prime consumer mortgage rates between 10 to $80 \mathrm{bps}$ of the $100 \mathrm{bps}$ decline in the MSR. Staff argued that this higher profitability would improve banks' scope for higher NPL provisioning and faster write-offs of delinquent loans. Staff analysis found that lowering NPLs does increase credit and economic growth (Annex 5).

\section{Staff urged ECCU member countries to implement other structural reforms that} would facilitate improving access to credit and reducing lending rates. In particular, staff noted that the regional foreclosure recommendations now being discussed by the Monetary Council should aim to reduce the time and cost of resolving problem loans and securing collateral. In certain jurisdictions (e.g., St. Lucia) this process averages in excess of five years. The establishment of a regional credit bureau would also reduce the problem of asymmetric information and lower the interest rate spread, thereby helping to support a revival of bank credit.

\section{Authorities' views}

\section{The ECCB and country authorities were willing to consider further reduction or} elimination of the MSR. However, they were concerned about the smaller pass-through of

\footnotetext{
${ }^{4}$ See 2015 ECCU Common Policies Staff Report; also Myrvoda, A., and Reynaud, J., "Monetary Policy Transmission in the Eastern Caribbean Currency Union", IMF Working Paper, forthcoming.

${ }^{5}$ A survey of 10 regional indigenous and foreign banks was conducted to collect interest rate data on prime mortgage and prime consumer rates on new loans.
} 
the lower MSR, as registered by the official statistics. To move the process forward, the ECCB asked for additional empirical evidence on the benefits of eliminating the MSR. Some country authorities also voiced their concern that in the absence of a fully functioning capital market, the elimination of the minimum savings deposit floor would severely limit the availability of financial instruments to the public.

\section{Global Withdrawal of Correspondent Banking Relationships (CBRs): A Need for Close Monitoring ${ }^{6}$}

Global withdrawal of CBRs is a risk to regional financial stability and cross-border payments systems. It will be important for the authorities to strengthen further AML/CFT and international tax cooperation frameworks and CIP governance and to find possible work-arounds to insulate themselves from a substantial loss of correspondent banking relationships.

\section{Background}

21. Global withdrawal of CBRs is a risk to financial stability and the real economy. Given the openness of ECCU countries, the availability of CBRs is crucial for the stability of the financial system and economic growth. On the other hand, for the large international banks, the relatively small volume of business generated from individual ECCU banks makes the risk-reward calculation of maintaining these relationships less attractive given the increase in regulatory fines and compliance procedures required by advanced country regulators.

22. Until now, the effects have had their most negative impact in the offshore banking sector. ${ }^{7}$ The loss of or inability to obtain CBRs has led to the revoking of some licenses and a significant decline in the volume of offshore banking license applications. ${ }^{8}$

\section{Thus far, onshore indigenous commercial banks have largely been able to manage the consequences of the withdrawal of CBRs but not without considerable cost and effort.} A survey of indigenous banks in the ECCU revealed that at the time of discussions none of the indigenous banks completely lost all CBRs with international banks. Over the past two years about half of indigenous commercial banks reported losing CBRs or receiving unofficial notifications of the forthcoming CBR terminations, but in most cases new replacements were found, often with smaller, less known (i.e., "second tier") banks. Thus, the effect of the withdrawal of CBRs thus far has been reflected mainly in rising correspondent banking fees, which in some cases doubled or tripled on some occasions. However, any further withdrawal by major

\footnotetext{
${ }^{6}$ See Annex 6 for a detailed account of the issues surrounding the withdrawal of CBRs.

${ }^{7}$ To assess the extent of the withdrawal of CBRs in the ECCU, staff surveyed 10 indigenous commercial banks and 5 regional offshore banking regulators, in some cases also supplemented by discussions. The surveyed commercial banks constitute about 55 percent of the locally incorporated bank assets in ECCU.

${ }^{8}$ Regulations in most jurisdictions require a licensed offshore bank to show proof of a CBR with a recognized international bank.
} 
correspondent banks from the region could be detrimental to indigenous banks' operations. In addition to cases of rising CBR fees, all domestic banks have been forced to dedicate more resources to due diligence and increased data reporting in order to maintain existing relationships. Discussions with the banking community suggest that the risk of loss of CBRs is negatively correlated with the size of the bank.

\section{Staff Recommendations}

\section{Staff encouraged the ECCB and country authorities to strengthen their regulatory} frameworks in order to allay any negative perceptions held by international banks. In particular, strong governance of CIPs and effective implementation of international AML/CFT standards to reduce financial integrity and security risks were crucial. ${ }^{9}$ The authorities should also accelerate CARTAC-supported BASEL II implementation and risk-based supervision in both the banking and non-banking sectors. Along the same lines, staff noted that, although costly, banks should increase information sharing with correspondent banks, which could ease their "know your customer's customer" concerns.

\section{To the extent that the risk of loss of CBRs is related to the small size of ECCU financial institutions, this would be an additional reason to consolidate the banking} system. Not only would the larger financial institutions provide a larger volume of business to correspondent banks but they could more efficiently handle the growing due diligence and oversight requirements to comply with international AML/CFT and tax cooperation standards. In the short run, staff suggested that the smaller indigenous institutions that seemed most at risk to the loss of CBRs should explore the possibility of paying the larger (indigenous and foreignowned) banks a fee to process their international transactions.

\section{Authorities' views}

26. Although banks have managed to find work-arounds thus far, the authorities were deeply concerned that the potential loss of CBRs could undermine macroeconomic and financial stability. As a result, they were actively addressing the issue on a number of fronts. They highlighted their strong and ongoing efforts in the AML/CFT and tax areas and argued that withdrawal of CBRs could not be justifiably based on concerns in those areas. At the CARICOM level, a technical task force has been set up, led by the Central Bank of Barbados, to undertake research and engage with advanced country regulatory institutions. Fund staff has been a contributor to this task force. A coordinated political strategy also is being developed, led by the Prime Minister of Antigua and Barbuda, to sensitize the authorities in the advanced countries to the adverse effect that the withdrawal of CBRs could have on the Caribbean. The authorities also noted that the current trends could also have the unintended

\footnotetext{
${ }^{9}$ FATF has launched a new round of mutual evaluations that will assess both whether the necessary laws, regulations or other required AML/CFT measures are in force and whether these AML/CFT systems are actually working.
} 
effect of undermining AML/CFT efforts as transactions would move away from established, regulated institutions into informal channels.

\section{The authorities asked that the Fund to continue to assist in finding possible}

solutions to the problem. They were pleased that the subject of the withdrawal of CBRs derisking would be a part of surveillance issues discussed with several advanced countries and also looked forward to the planned conference that the Managing Director will host in September. They also informed staff that the task force was exploring the feasibility of establishing a Caribbean non-deposit taking financial institution in the United States, through which banks from the Caribbean would conduct transactions with U.S. entities.

\section{Strengthening Fiscal Policy in the context of high debt, low fiscal buffers, and increasing natural disasters}

The necessary measures to achieve the ECCU debt target of 60 percent of GDP by 2030 have been the focus of the bilateral Article IV consultations in the past year. The Common Policies discussions focused on three inter-related themes: mainstreaming natural disasters in macroeconomic policy frameworks; avoiding over-reliance on citizenship revenues for funding recurrent budgetary operations; and improving the fiscal framework to underpin the fiscal consolidation effort.

\section{Background}

28. The ECCU is one of the most vulnerable regions in the world to natural disasters. Yet governments do not internalize the costs in their policy frameworks. The probability of an ECCU country being hit this year by a natural disaster is close to 25 percent, with damages of about 26 percent of GDP (average damage per disaster for ECCU countries over the period 19802015)..$^{10}$ Against this, the only systematic insurance that governments have is their participation in the Caribbean Catastrophic Risk Insurance Facility (CCRIF), whose average payout is less than 0.5 percent of GDP, mainly because relatively little coverage has been purchased. Homeowners and financial institutions appeared better protected against natural disasters. Banks require borrowers to

\begin{tabular}{|c|c|c|c|}
\hline \multicolumn{4}{|c|}{$\begin{array}{l}\text { ECCU: Probability, Annual Damages, and Esimtated Damages } \\
\text { (Probability: percent. Damages: percent of GDP, period: 1990-2014) }\end{array}$} \\
\hline & \multicolumn{2}{|c|}{$\begin{array}{l}\text { Probability of } \\
\text { at least } 1 \text { Disaster in }\end{array}$} & \multirow{2}{*}{$\begin{array}{c}\text { Ave. } \\
\text { disaster } \\
\text { damages }\end{array}$} \\
\hline & 1 year & 5 years & \\
\hline Antigua and Barbuda & 24.4 & 75.3 & 23.4 \\
\hline Dominica & 24.4 & 75.3 & 27.3 \\
\hline Greneda & 18.1 & 63.2 & 41.1 \\
\hline St. Kitts and Nevis & 14.8 & 55.1 & 42.7 \\
\hline St. Lucia & 33.0 & 86.5 & 12.0 \\
\hline St. Vincent and the Grenadines & 27.4 & 79.8 & 8.7 \\
\hline
\end{tabular}
purchase hurricane insurance as a condition for providing mortgage loans; and local insurance companies are required to re-insure 75 percent of their exposure with international companies (Annex 8).

10 "Gone with the Wind: Estimating Hurricane and Climate Change Costs in the Caribbean", Acevedo, IMF Working Paper No. 16/199. 


\section{Countries need stronger fiscal} policies to achieve the debt target. As noted earlier, ECCU countries have made some progress with much-needed fiscal consolidation though, in staff's view, still insufficient. In February 2015, the ECCB Monetary Council (comprising all Ministers of Finance) established a regional debt target of 60 percent of GDP to be achieved by 2030 and mandated all members to elaborate medium-term fiscal adjustment plans to achieve the target. Some of the countries with high debt levels have

\begin{tabular}{|l}
\hline Fiscal Adjustment Scenarios in Selected ECCU Countries \\
(In percent of GDP)
\end{tabular}
outlined fiscal programs (Antigua and Barbuda, Grenada, and Dominica) and will need to steadfastly implement them. St. Lucia and St. Vincent and The Grenadines are yet to elaborate such programs.

30. Inflows from Citizenship-by-Investment programs offer an opportunity to facilitate debt reduction and build buffers against future natural disasters. However, ECCU CIPs face increased competition both from among themselves and from outside the region, particularly Malta's newly launched CIP and other residency-by-investment schemes in Europe. In addition, rising global migration pressures, elevated security concerns, and geopolitical tensions may trigger adverse actions by the international community, in particular the suspension of visa-free travel for citizens of CIP countries.

\section{Staff Recommendations}

\section{Staff reiterated past advice to strengthen the fiscal framework by following} Grenada's example and passing national fiscal responsibility legislation that would enshrine the regional debt target and specify fiscal rules. These initiatives would significantly enhance the credibility of the debt target as the fiscal anchor for the region. In this context, staff also urged the governments to move quickly to articulate medium-term fiscal adjustment strategies in next year's budgets, focused on the primary balance target excluding CIP revenues.

32. The country authorities should internalize the expected costs of natural disasters in their macro-fiscal frameworks, including building the necessary fiscal buffers. Assuming that half of total natural disaster costs fall on the government, staff's analysis suggests that, on average, ECCU governments would need to save approximately 1 percent of GDP per year (i.e., target a higher primary surplus by this amount) to pay for its share of damages from the most expensive disaster. Staff noted that it would be appropriate to include escape clauses in fiscal rules that would allow temporary suspension of the rules in the event of a natural disaster.

33. Beyond the adoption of fiscal rules, staff encouraged the country authorities to continue to improve the overall public financial management (PFM) framework (Annex 7). 
This year, staff recommendations focused on two areas: CIPs and public sector investment management.

- Accounting practices should adopt a broader fiscal perimeter to facilitate analysis of the true fiscal policy stance. In particular, all CIP revenues to the public sector, and related expenditure, should be recorded on budget. Staff pressed for comprehensive and consistent reporting of CIP revenues in the external and fiscal accounts across countries. In St. Kitts and Nevis, contributions to the Sugar Industry Diversification Foundation (SIDF) - the country's NDF-are not reported on budget, while in Antigua and Barbuda, only NDF resources are managed on budget with the CIP Unit surplus from application fees to government are managed at Cabinet's discretion, outside the budget framework. ${ }^{11}$ Similar information gaps exist in Dominica. Funding fiscal or quasi-fiscal operations from outside the budget weakens the assessment of the underlying fiscal stance and provides incomplete information on the actual size of governments' spending commitments.

- A significant portion of CIP resources should be saved in a fund to address costly natural disaster shocks and finance disaster resilient infrastructure (Annex 8). Countries would need saving fund stocks in the range of 6 12 percent of GDP to self-insure against natural disasters, and annual savings in years with no natural disaster of 0.4-1.9 percent of GDP to in order to ensure fund's financial sustainability with a low probability of depletion. Staff continues to urge governments to use CIP revenue

\begin{tabular}{|c|c|c|c|c|c|c|}
\hline \multicolumn{7}{|c|}{$\begin{array}{l}\text { Government Saving Funds for Natural Disasters (ND) in the ECCU } \\
\text { (In percent of GDP unless otherwise indicated) }\end{array}$} \\
\hline & ATG & DMA & GRD & KNA & LCA & VCT \\
\hline Fund size & 12.00 & 10.00 & 6.00 & 10.00 & 8.00 & 8.00 \\
\hline Annual budget saving if no ND & 1.90 & 1.50 & 0.35 & 1.90 & 1.05 & 0.95 \\
\hline Probability of fund depletion, units & 0.05 & 0.08 & 0.04 & 0.07 & 0.06 & 0.03 \\
\hline Annual expected use of the fund if ND & 8.7 & 5.9 & 3.0 & 4.8 & 3.4 & 2.6 \\
\hline Average fiscal cost of simulated ND 1/ & 57.9 & 29.3 & 29.7 & 19.3 & 13.7 & 12.9 \\
\hline \multicolumn{7}{|c|}{$\begin{array}{l}\text { Source: Staff calculations based on authorities' data. } \\
1 / \text { Includes the estimated average decline in revenues and increase in primary expenditures in the simulations, } \\
\text { net of the expected increase in donor grants. }\end{array}$} \\
\hline
\end{tabular}
windfalls to the public sector to reduce the public debt load, build precautionary buffers and support strategic public investment projects in their economies, where appropriate. Funding capital projects using CIP resources should ensure rigorous project evaluation and selection and the existence of sufficient resources to finance projects to completion, to limit potential contingent liability to government.

- Country authorities will have to improve substantially their capacity to design and manage public sector investment programs (PSIPS). The tight fiscal space, coupled with the large gaps in basic infrastructure, including in the transport and power sectors and natural disaster resilience, puts a premium on well-designed and implemented capital budgets. Staff recommended that authorities seek Fund TA in this area. A clearly articulated PSIP and

\footnotetext{
${ }^{11}$ In St. Kitts and Nevis, efforts are ongoing to improve transparency of the SIDF financial reporting and integrate it with the government's consolidated fund. In Antigua and Barbuda, the authorities have agreed to implement a mechanism to transfer a share of the CIP Unit surplus to the consolidated central government account as part of the proposed fiscal consolidation program in 2016.
} 
demonstrated technical capacity will put governments in a better position to access donor funds that support renewable energy investments and climate resilience projects.

\section{A comprehensive governance framework is crucial to mitigating increased risks}

facing CIPs. Staff urged the authorities to develop a regionally accepted set of principles and code of conduct that would establish benchmark standards for the region's CIPs. This was important to prevent negative shocks to the reputation of one CIP from spilling over to other programs in the region and to avoid destructive competition among the individual CIPs. ${ }^{12}$ Staff reiterated past advice on the crucial importance of a rigorous due diligence process for applicants. Staff also recommended more transparency, in particular that the number of citizenships granted, revenues earned, and their use (including the amounts saved, spent or invested), should be made public and be subject to financial audits.

\section{Authorities' views}

\section{Country authorities reiterated their commitment to the debt target but were not ready to endorse staff's proposal to adopt fiscal rules. However, the ECCB governor} expressed support for the proposal, noting that he had made a similar recommendation to country authorities. Authorities strongly agreed with the need to have fiscal buffers to address natural disasters. In this context, the Dominican authorities noted their plans to establish a Vulnerability Resistance Fund, using a portion of CIP revenues. The St. Kitts and Nevis authorities are also establishing a Growth and Resiliency Fund, to manage CIP inflows and build buffers for resilience to shocks. Country authorities generally supported staff's proposals to enhance CIP management and, in particular, agreed to move forward on establishing common principles for regional CIPs.

\section{E. Structural Reforms: A Menu of Options to Increase Competitiveness}

Real effective exchange rate and current account assessments are sensitive to recent provisional data revisions and methodology changes and do not allow for definitive conclusions at this time. However, non-price indicators point to weak competitiveness in the region. International reserves are broadly adequate according to most metrics. Fiscal consolidation and structural reforms aimed at lowering the cost of doing business, improving resilience to natural disasters, increasing labor productivity, and broadening regional collaboration are crucial to improving competitiveness and potential growth.

\footnotetext{
12 For example, staff suggested that the authorities share due diligence information on clients to prevent clients from shopping among the various programs in the event that their citizenship application is rejected by a given jurisdiction.
} 


\section{Background}

36. Despite an appreciation since the second half of 2014, the ECCU real effective exchange rate has not changed much over the last six years. The customer-based REER is not significantly higher than in previous years, since the ECCU dollar appreciation has followed the appreciation of the US\$ (the United States is the region's main trading partner). Similarly, the competitor-based REER is only 4 percent higher than at its low point in 2012 and 0.6 percent below its 10 -year average. The estimated REER misalignment varies significantly

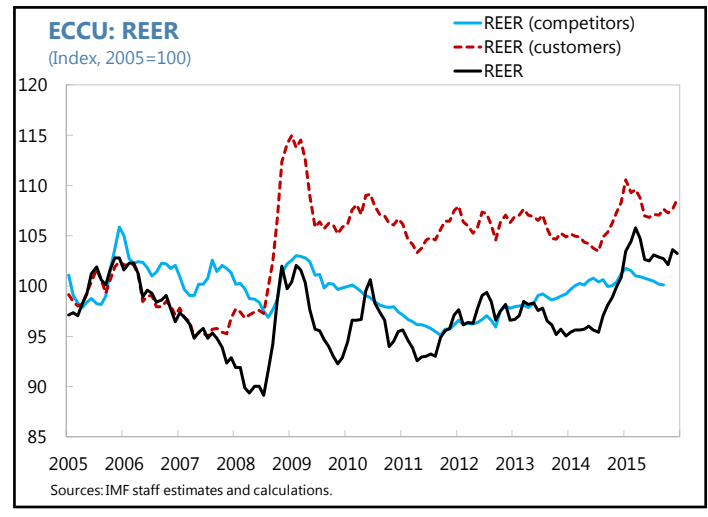
depending on whether the provisional revised external sector statistics are used (Appendix 11)

37. Non-price indicators point to low competitiveness in the region. The region's market share in Caribbean tourism has been declining in recent years, while the region's place in investment climate rankings has been worsening (Figure 5). Labor market factors erode competitiveness, as there is evidence of labor market rigidities (Annex 9), weak productivity, and rising unit labor costs, which have grown substantially relative to the United States. Some ECCU member jurisdictions seem to be adjusting through high unemployment, rather than elevated prices. High energy costs also adversely affect competitiveness in the region as does the susceptibility and lack of resilience to natural disasters, which have contributed to growing infrastructure gaps (Annex 10).

\section{Staff Recommendations}

38. Staff followed up on past advice aimed at boosting competitiveness, in particular in the areas of resilience to natural disasters, energy policy, regional collaboration, and labor market policies. As described earlier, CIP-funded saving funds and a strengthened PSIP should be a part of a strategy to build resiliency-enhancing infrastructure. On energy policy, staff commended the country authorities on the progress in implementing their various energy plans, which have the potential to be a linchpin for stronger future economic growth. ${ }^{13}$ For example, in St. Vincent and the Grenadines, the authorities continue to move ahead, together with two strategic private partners and financial and technical assistance from the CDB, to implement their geothermal development project, scheduled to come on line in 2018. This project would increase the energy mix to 80 percent renewables from 12 percent currently and reduce electricity costs to the consumers to US $\$ 0.20$ per kilowatt hour from US $\$ 0.40$ in 2015 . Research by staff indicates that successful implementation of the countries' energy plans (at an average cost of 7 percent of

\footnotetext{
${ }^{13}$ See 2014 ECCU Common Policies Staff Report (at imf.org), Appendix IV: Energy Sector Challenges and Policies in the ECCU; and WP/16/53, Caribbean Energy: Macro-related Challenges.
} 
2015 GDP) could raise real GDP by 4 percent and without jeopardizing public debt sustainability (Annex 10).

\section{Staff noted a couple of positive developments in regional collaboration and} encouraged the authorities to continue to move forward in this area. In particular, staff commended new efforts aimed at developing a single domestic space. Officials have started to discuss what systems will be required for harmonized border management. Thus, for example, visitors arriving from outside the ECCU would satisfy all border control checks only at the first point of entry and then be able to move freely within the region. Staff noted that such a reform could significantly enhance the competitiveness of ECCU tourism by facilitating multi-destination travel. At the same time, staff warned that the ongoing problems plaguing intra-regional travel and the loss-making regional airline will need to be solved. In other areas, staff urged authorities to follow through on initiatives to implement a single regional regulatory authority for nonbanks. Stakeholders have provided comments on the draft legislation covering the first phase of this project, a single regulator for insurance companies.

\section{Staff continued to press for the adoption of policies that would reduce unit labor} costs and boost employment (Box 2). Toward this end, staff urged reforms to better align real wage growth with productivity growth, including increasing labor market flexibility and controlling public sector wage growth. Staff's analysis suggested that public sector wages have a demonstration effect on private sector wages. Staff recommended additional measures focused on increasing productivity, such as strengthening labor-training programs, especially for youth and displaced agricultural workers. (Annex 9).

\section{Staff once again highlighted the importance of improving macroeconomic}

statistics. In this context, staff welcomed the Statistics Canada-led TA project to strengthen labor market statistics. The revisions to the BOP statistics now underway are welcome and staff offered additional CARTAC and STA TA to ensure that the revisions are properly vetted. Staff noted that just as there had been a need to account properly for offshore medical schools, the treatment of CIP flows also should be reviewed to ensure consistency with BPM6. ${ }^{14}$ Staff noted concerns expressed by officials at some national statistics agencies about the lack of sufficient technical capacity and suggested to the authorities that they explore the feasibility of a regional statistical agency, which could optimize the use of scarce human resources.

\section{Authorities' views}

42. The authorities expressed a strong commitment to growth-enhancing reforms and pointed to various initiatives underway aimed at reducing the cost of business. They were upbeat about the economic potential for the energy sector reforms underway and noted that they were working closely with the CDB to ensure the projects were well designed and

\footnotetext{
${ }^{14}$ For example, in St. Kitts and Nevis, staff has adjusted the official BOP statistics to account for misclassification and exclusion of some CIP flows.
} 


\section{Box 2. Explaining High Unemployment in the ECCU}

Labor market data in the ECCU, although scarce, indicate relatively high unemployment levels in some countries especially in the years following the global financial crisis. While unemployment varies among countries, it is very high in Grenada, St. Lucia, and St. Vincent and the Grenadines. Aggregate employment in the ECCU has remained stagnant since the global crisis, while private sector employment has declined significantly.
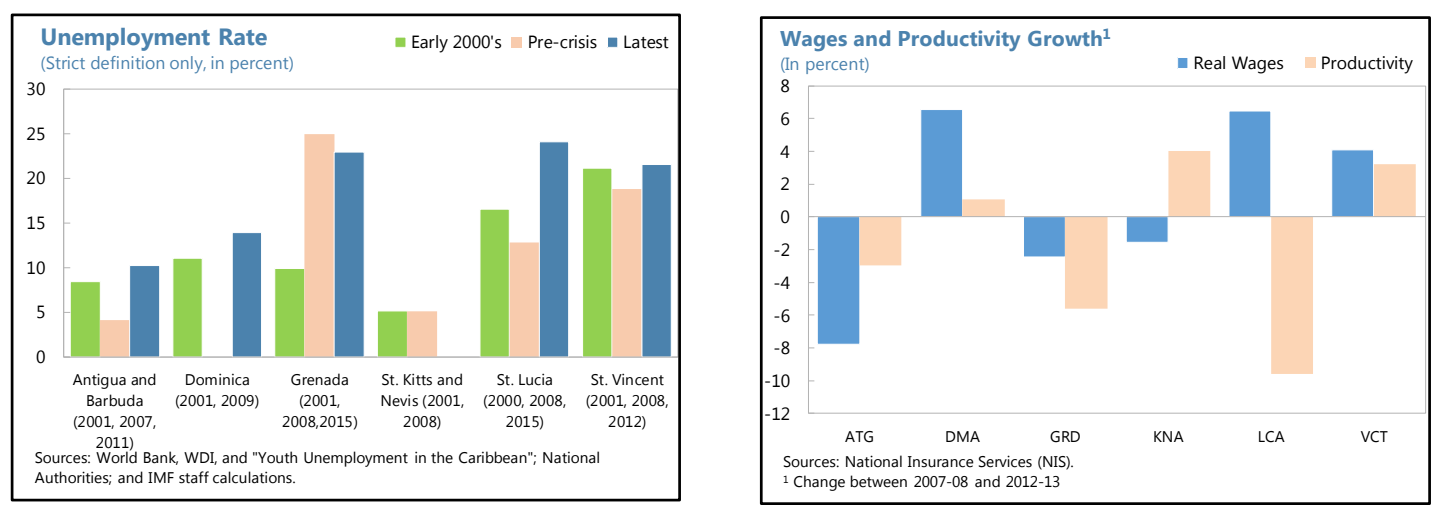

The large disconnect between continued real wage growth and stagnant productivity in Grenada and St. Lucia is likely a contributory factor to their very high unemployment rates. This disconnect could be partly the result of strong labor union activity and negotiation through collective bargaining process in these countries, especially in the public sector. There is also quantitative evidence based on Grenada's employment data that raising public sector wages contributes to raising labor costs across sectors, through demonstration effects, as

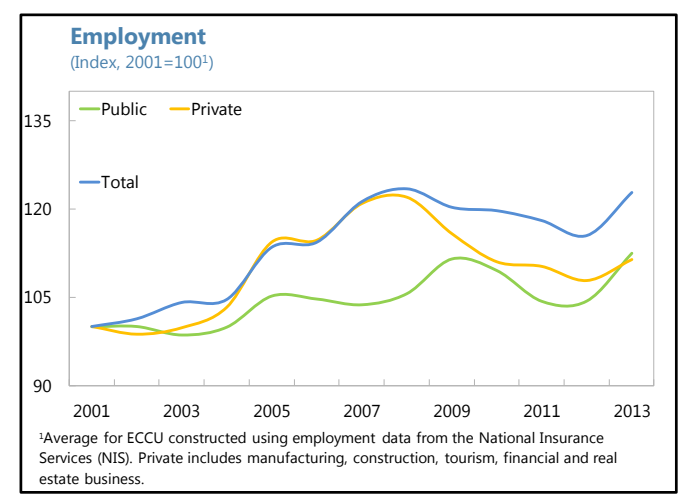
private sector unions often negotiate wages using public sector wages as a benchmark.

Other factors, such as high reservation wages, long-term sector trends, and natural disasters may also contribute to structural unemployment and require further analysis. Relatively higher salaries in the tourism and public sector have increased the reservation wage. The impact of natural disasters on the laborintensive agriculture sector and the trend contraction in this sector especially since decline of the banana industry may also have increased structural unemployment, particularly in Grenada. See Annex 9 for details.

economically viable. While supporting enhanced regional collaboration, authorities felt these should not undermine strong national preferences where such existed. At the same time, they noted that technical work was underway to study the legal and regulatory requirements to establish a customs union to facilitate the free movement of goods throughout the region. On statistics, the authorities noted that the Job Opening and Labor Turnover Survey (JOLTS), which 
collects data on jobs, separations, hires, separations, and vacancies, has been piloted in three countries. They saw the survey as providing invaluable input for designing labor market policies.

\section{STAFF APPRAISAL}

43. The economic recovery in the ECCU is continuing, albeit at a relatively slow pace, supported by growth in the United States and low global oil prices. For 2016, staff forecasts growth at 2 percent, rising to its estimated potential rate of $2 \frac{1}{2} 2$ percent next year based mainly on projected moderate growth in the tourism sector. At the same time, unemployment rates are unacceptably high, especially among youth. Inflation is expected to remain low and the external current account deficit is projected to narrow to its smallest level since the late 1990s, owing to the favorable global environment. Risks in the short run appear balanced but the region still faces many vulnerabilities, in particular related to high public debt, a fragile banking system, susceptibility to natural disasters, and weak competitiveness.

44. The authorities have taken decisive action to strengthen the banking system, but the tasks are not finished. The Banking Act greatly improves the bank resolution and supervision framework, while the Eastern Caribbean Asset Management Company (ECAMC) Act now needs to become operational. The successful resolution of three insolvent banks eliminates a major risk to regional financial stability, and returns normalcy to bank operations for the first time since $2011^{15}$. Bank mergers should be explored to ensure the viability of the indigenous banks and reduce the likelihood that they will suffer from the withdrawal of CBRs by international banks.

45. While indigenous commercial banks are managing the consequences of global withdrawal of CBRs, offshore banks face more serious consequences. This has led to the revoking of some licenses in the offshore banking sector and a significant decline in the volume of license applications. The authorities need to strengthen further their citizenship program management and AML/CFT and international tax cooperation frameworks in order to allay any negative perceptions held by international banks. Banks should pro-actively increase information sharing with correspondent banks and explore work-arounds as needed. The authorities should continue to engage with authorities in advanced countries to sensitize them to the serious potential risks to local economies from a further loss of correspondent banking services.

\section{The ECCB should eliminate the minimum saving deposit rate and remove} distortions that have contributed to a protracted decline in credit and a buildup of excess liquidity. The lowering of the MSR last year has led to some reduction in lending rates, and banks have seen some increase in their net interest margin, which improves capital positions.

47. Fiscal performance has improved, but more adjustment is needed. Staff welcomes the improvement in underlying fiscal positions across most ECCU countries and the decline in

${ }^{15}$ Following a period of financial difficulties, the ECCB assumed control of ABIB under its emergency powers in July, 2011. 
public debt. However, except in St. Kitts and Nevis and Grenada, achieving the regional debt target by 2030 will require additional fiscal adjustment measures. All country authorities should move quickly to comply with the Monetary Council's 2015 decision to specify medium-term fiscal adjustment strategies to achieve the debt target. Fiscal programs should include building in extra savings to cover the expected costs of from natural disasters.

48. The credibility of the medium-term fiscal adjustment strategies would be enhanced by strengthening the underlying fiscal framework. Like Grenada in 2015, other ECCU countries are encouraged to pass national fiscal responsibility legislation to enshrine the regional debt target and specify fiscal rules. Governments need to improve their capacity to manage public sector investment programs (PSIPs).

49. Other public financial management reforms, particularly related to citizenship-byinvestment programs (CIPs), are also important. These include reflecting all CIP-related revenues and expenditures in the fiscal accounts; and saving the bulk of the resources in a wellmanaged fund to address costly natural disaster shocks and finance disaster resilient infrastructure. The authorities should also develop a regionally accepted set of principles and code of conduct for the region's CIPs.

50. The ECCB continues to follow policies consistent with achieving its mandate of ensuring the stability of the currency. The fixed exchange rate regime, with an unchanged peg to the U.S. dollar since 1976 is sustainable. Real effective exchange rate and current account assessments are sensitive to recent statistical methodological changes and do not allow for definitive conclusions at this time. However, non-price indicators, including high unemployment in some countries, point to weak competitiveness in the region. International reserves are broadly adequate according to most metrics.

51. Structural reforms aimed at lowering the cost of doing business, improving resilience to natural disasters, reducing unit labor costs, and broadening regional collaboration are crucial to improving competitiveness and potential growth. The initiatives under way to reduce energy costs and exploit renewable energy capacity need to be implemented carefully and safeguard debt sustainability. The authorities should deepen regional collaboration efforts to overcome technical capacity constraints and exploit economies of scale. In particular, staff supports plans to create a single domestic space that would allow for unconstrained tourist travel throughout the ECCU.

52. Data issues: Macroeconomic statistics, especially in the BOP and labor market areas, as well as in government finance and public sector debt, need to be improved to allow adequate surveillance and policy analysis. The authorities are urged to follow through on recent welcome initiatives.

53. The discussions with the ECCU authorities will be on the 12-month cycle in accordance with Decision No. 13655-(06/1), as amended. 


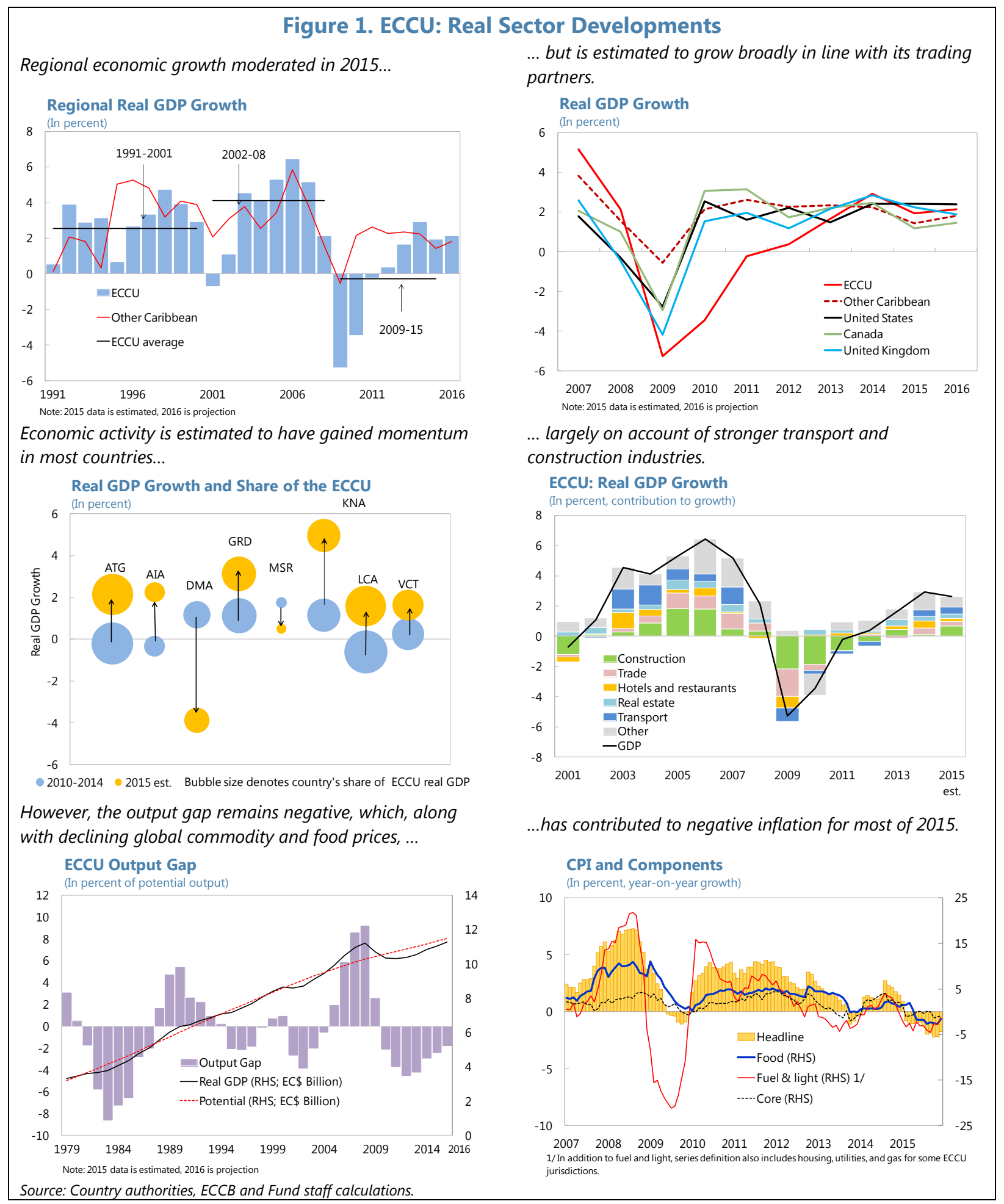


Figure 2. ECCU: Tourism Developments

Tourism receipts comprise a major portion of total ECCU exports.

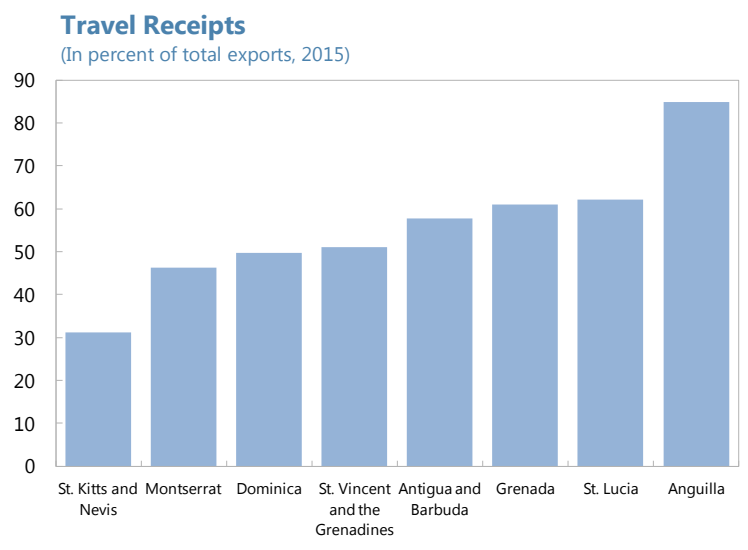

The source market is undiversified with almost $3 / 4$ of tourists coming from North America and the U.K.

Inbound Tourism: by Source Market (In percent, 2015)

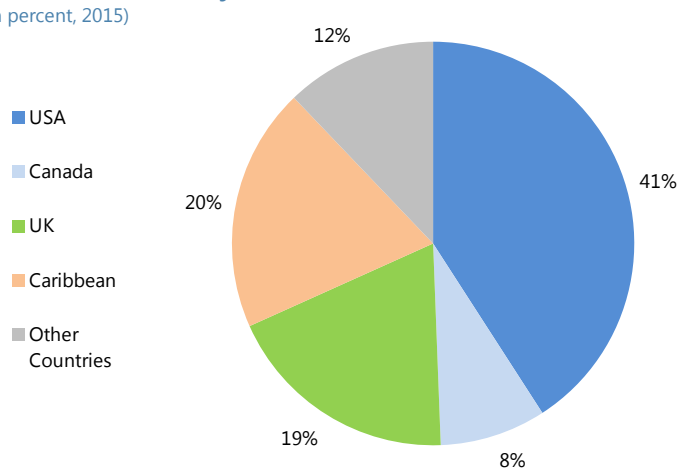

Travel revenues have been growing with the recovery in the US...

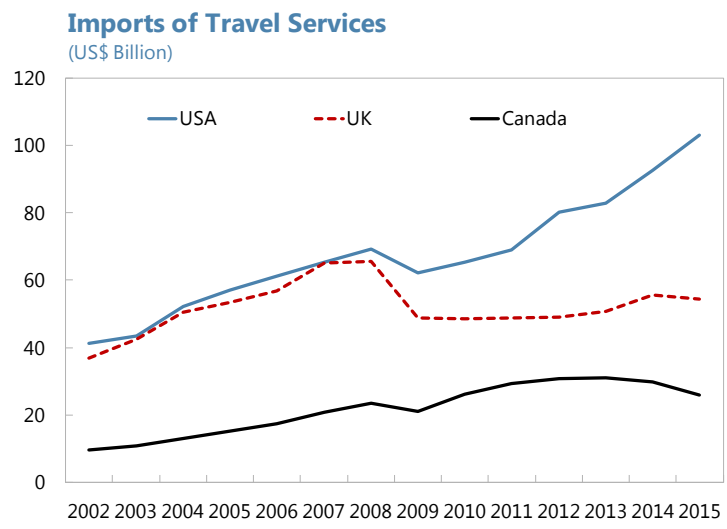

... but ECCU's share has continued to fall.

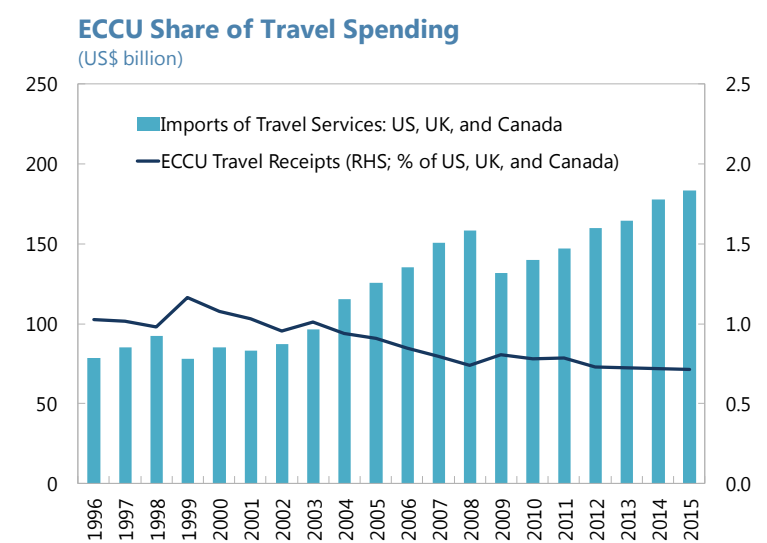

Tourist arrivals growth declined somewhat in 2015, largely on account of Canadian travelers,...

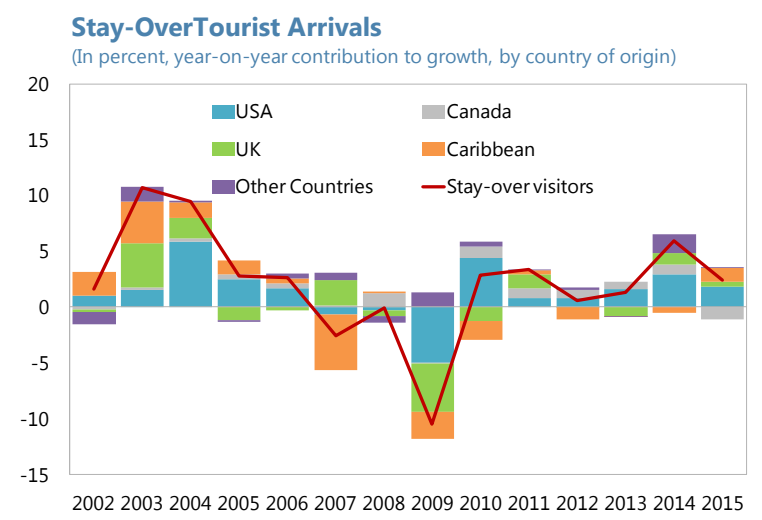
...also accompanied by a sharp deceleration in visitor expenditures.

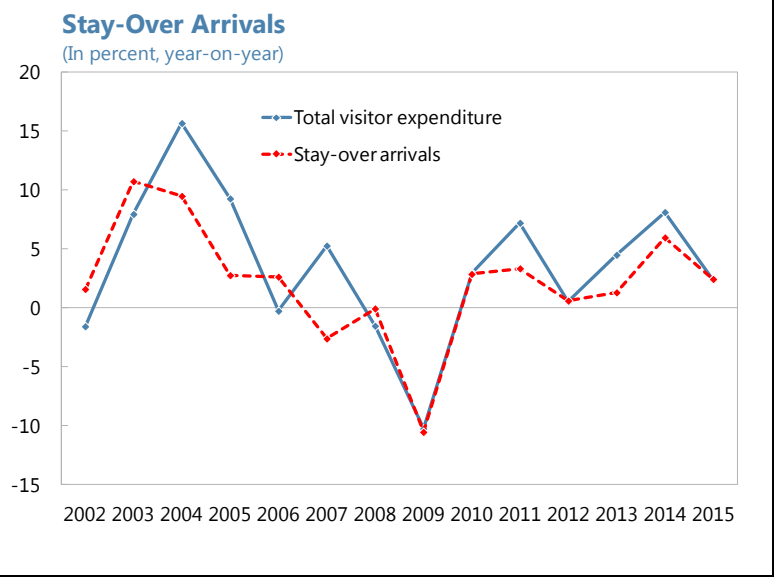


Figure 3. ECCU: Monetary Developments

Excess reserves remain elevated in most jurisdictions ...

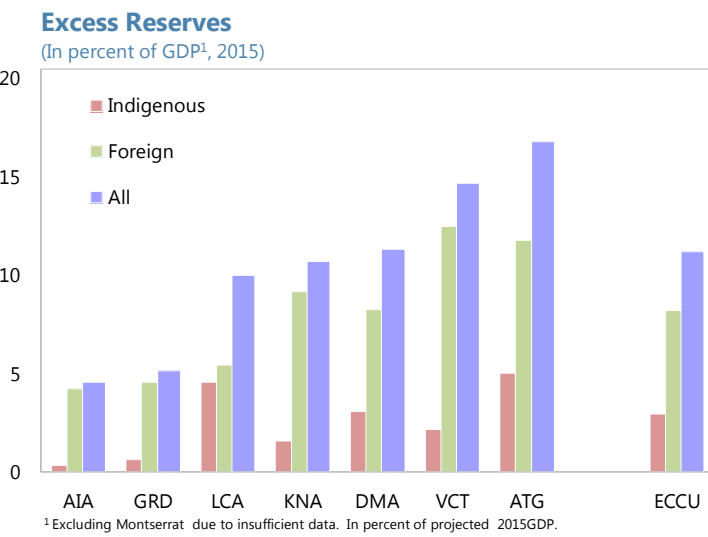

This reflected robust deposit growth for most of 2015, despite the sharp decline in indigenous banks' deposits in early 2016,...

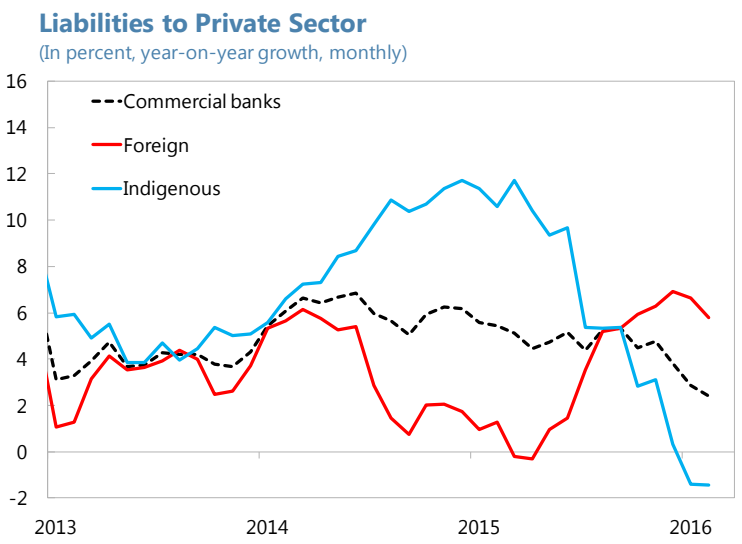

Robust reserve money growth, amidst restrained broad money increase, put downward pressure on the money multiplier.

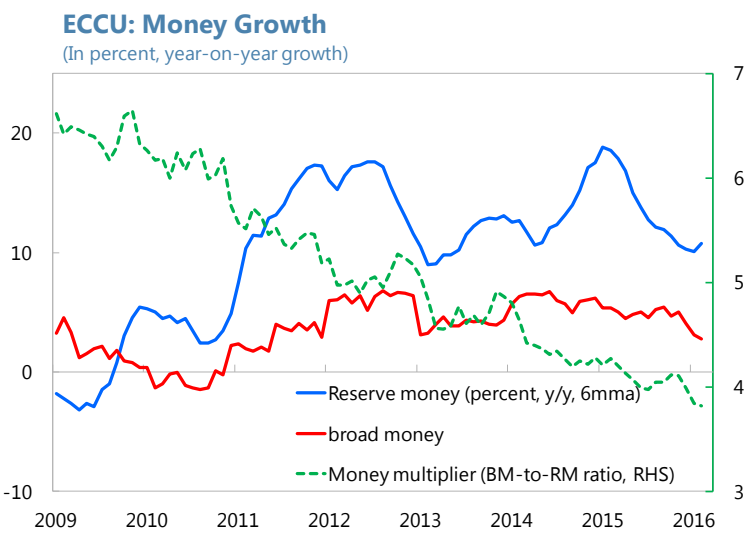

... and reached historically high levels in 2015.

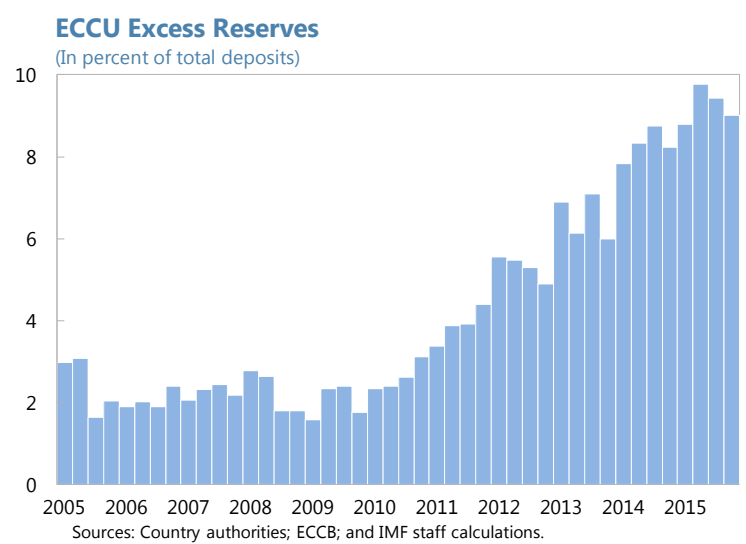

... and continued credit contraction.

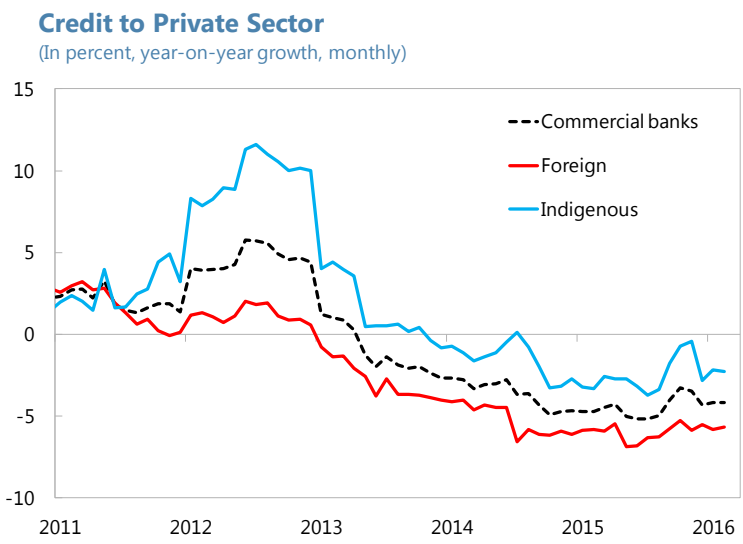

Monetary policy remained passive, while average deposit rates fell below the minimum saving deposit floor rate.

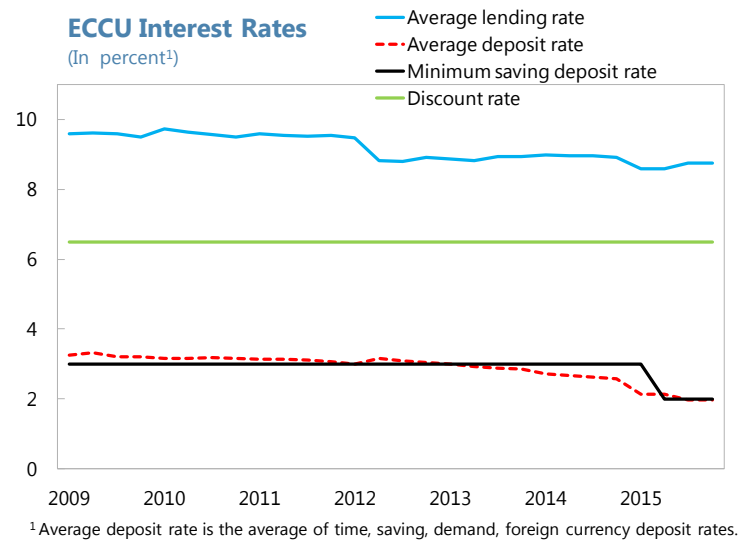


Figure 4. ECCU: Financial Soundness Indicators

NPL ratios edged down to 17 percent at end-2015.

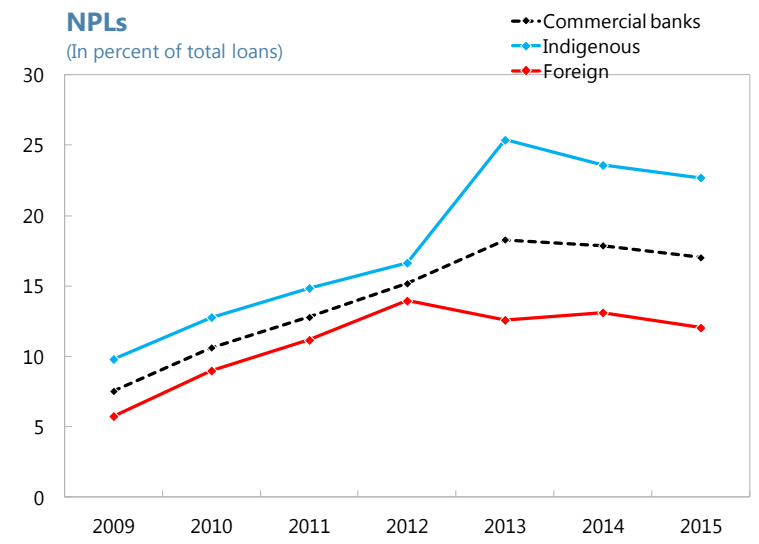

Meanwhile, profitability picked up in 2015, in part on the back of the decline in the minimum savings deposit rate....

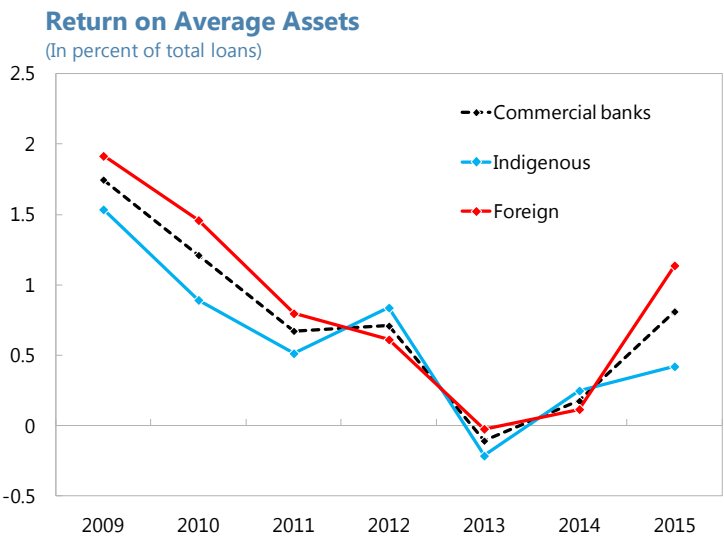

Excess liquidity has continued to provide a boost to banks' liquidity ratios....

Commercial Banks' Liquidity

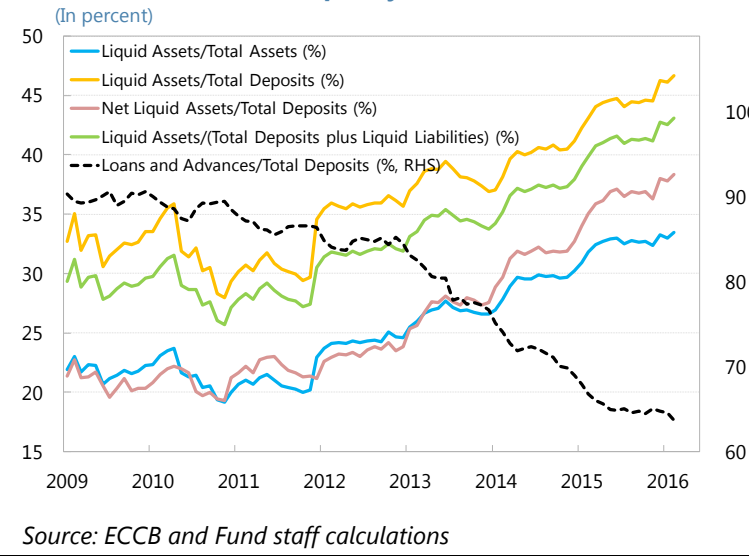

NPL provisioning continued to increase among the foreign banks.

Provisions to NPLs

(In percent, provisions to loan losses to non-performing loans)

50

-...Commercial banks

$=-$-Indigenous

$-\bullet-$ Foreign

40

30

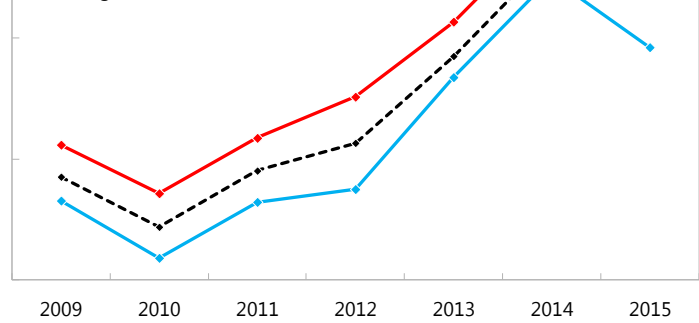

... along with slight improvements to capital adequacy.
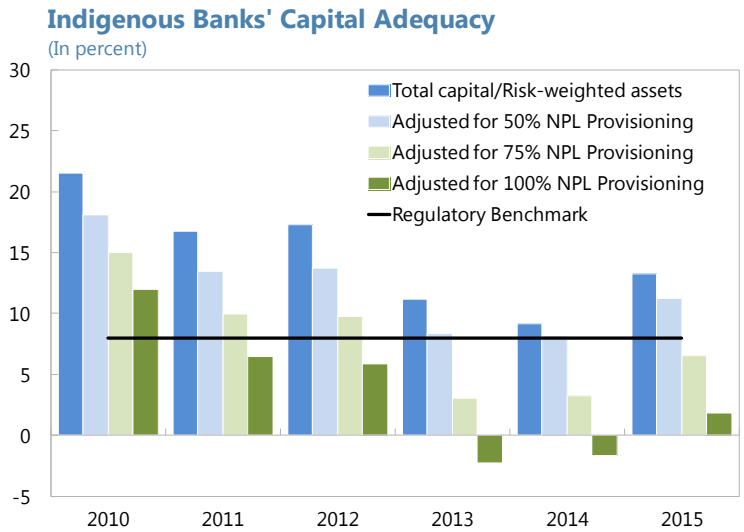

... particularly among foreign banks.

Cash Reserves / Total Deposits (In percent)

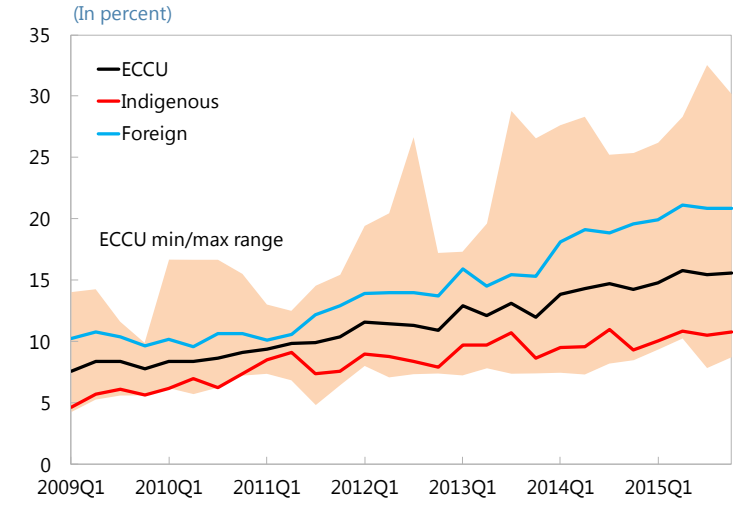




\section{Figure 5. ECCU: Doing Business Indicators ${ }^{1}$}

ECCU countries continue to exhibit large disparities in the ease of doing business, despite being relatively similar economies.

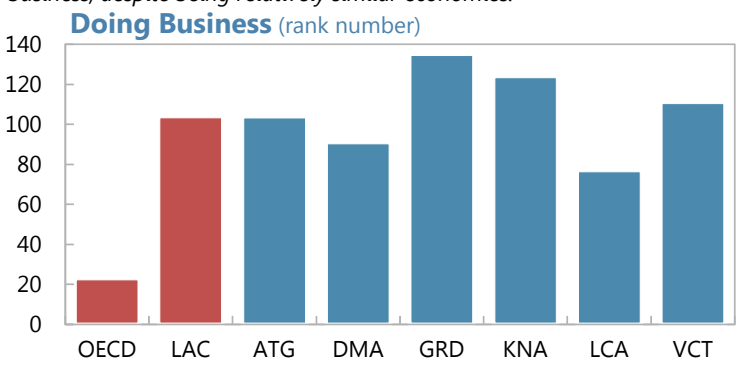
Registering property remains difficult, with costs far above the $L A C$ average in some jurisdictions.

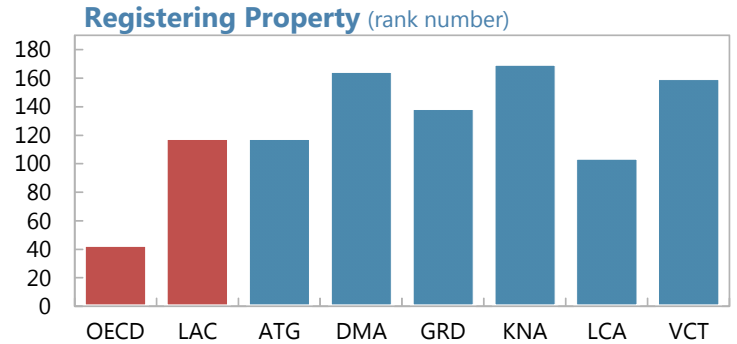

Dismissal costs are ranked the highest,...

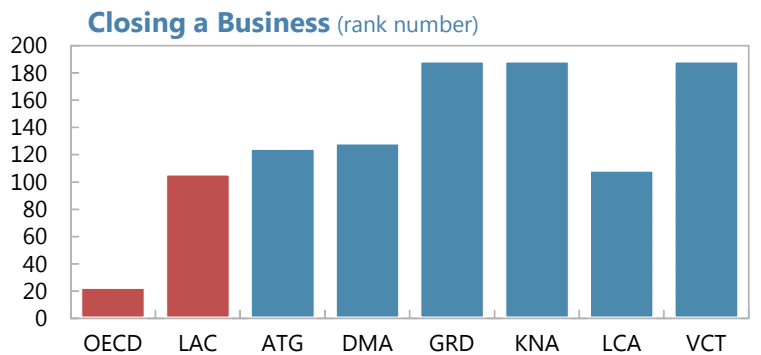

While in most ECCU countries setting up a business can be done with relative ease, a number of ECCU economies still lag behind.

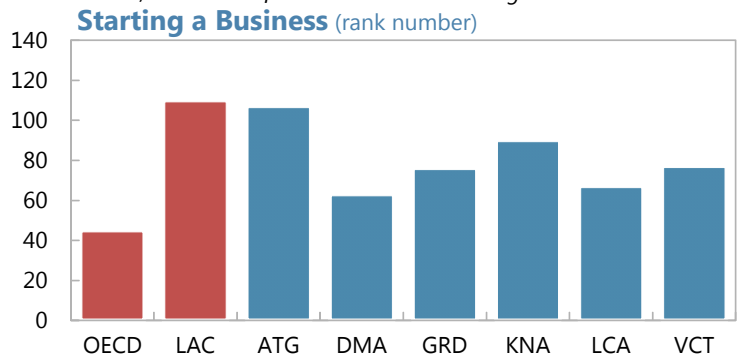

...while difficulties obtaining credit exist in all members due to a lack of credit information and less than effective collateral and bankruptcy laws.

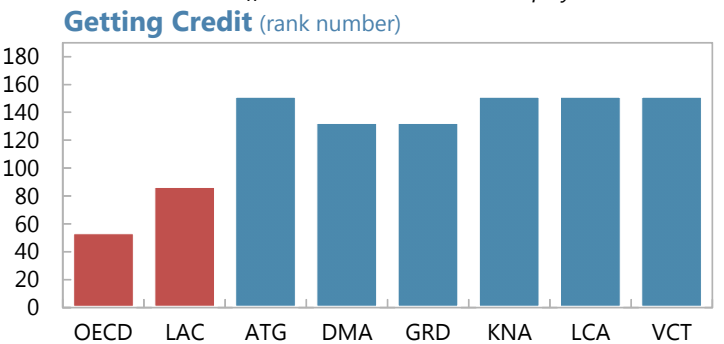

... while the costs of commercial contract enforcement remains largely on par with advanced countries.

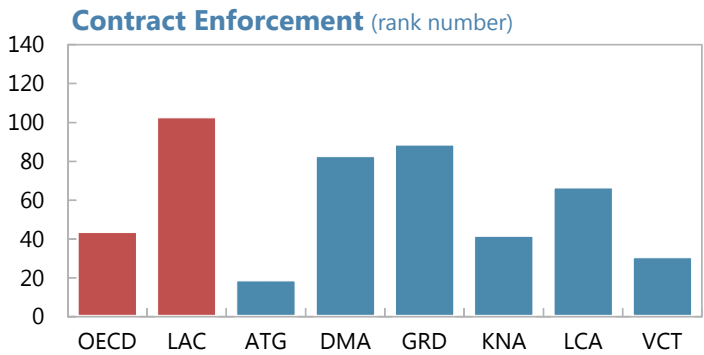

Doing Business (rank number)

ECCU countries have seen a worsening of their Doing Business ranking 2/.

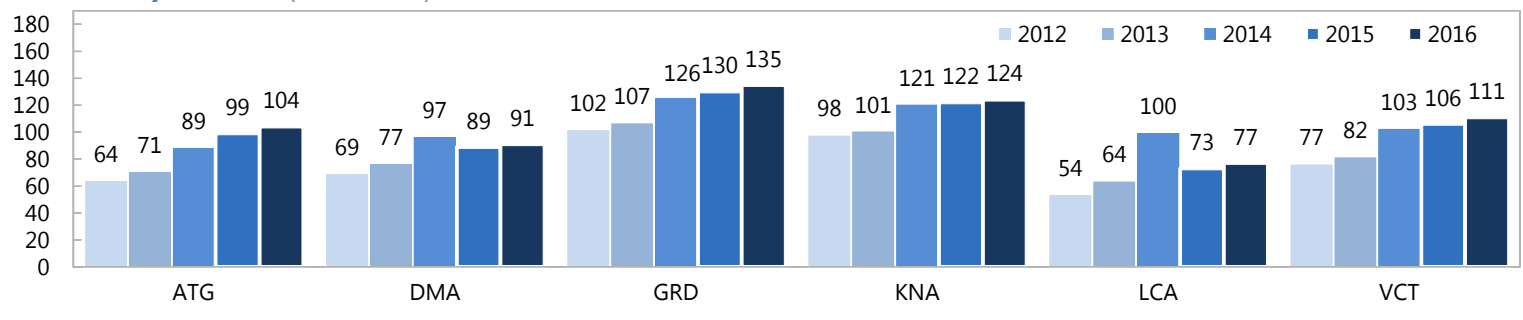

Sources: World Bank 2016 Doing Business Indicators; and Fund staff calculations.

1/ Smaller numbers represent greater ease in doing business. 2016 Doing Business rankings are across 189 countries. These indicators should be interpreted with caution due to a limited number of respondents, a limited geographical coverage, and standardized assumptions on business constraints and information availability.

2/ Annual rankings are rebased to adjust for different sample sizes.

Note: Antigua and Barbuda (ATG), Dominica (DMA), Grenada (GRD), St. Kitts and Nevis (KNA), St. Lucia (LCA), St. Vincent and the Grenadines (VCT), and Latin America and Caribbean (LAC). 
Table 1. ECCU: Selected Economic and Financial Indicators, 2009-21

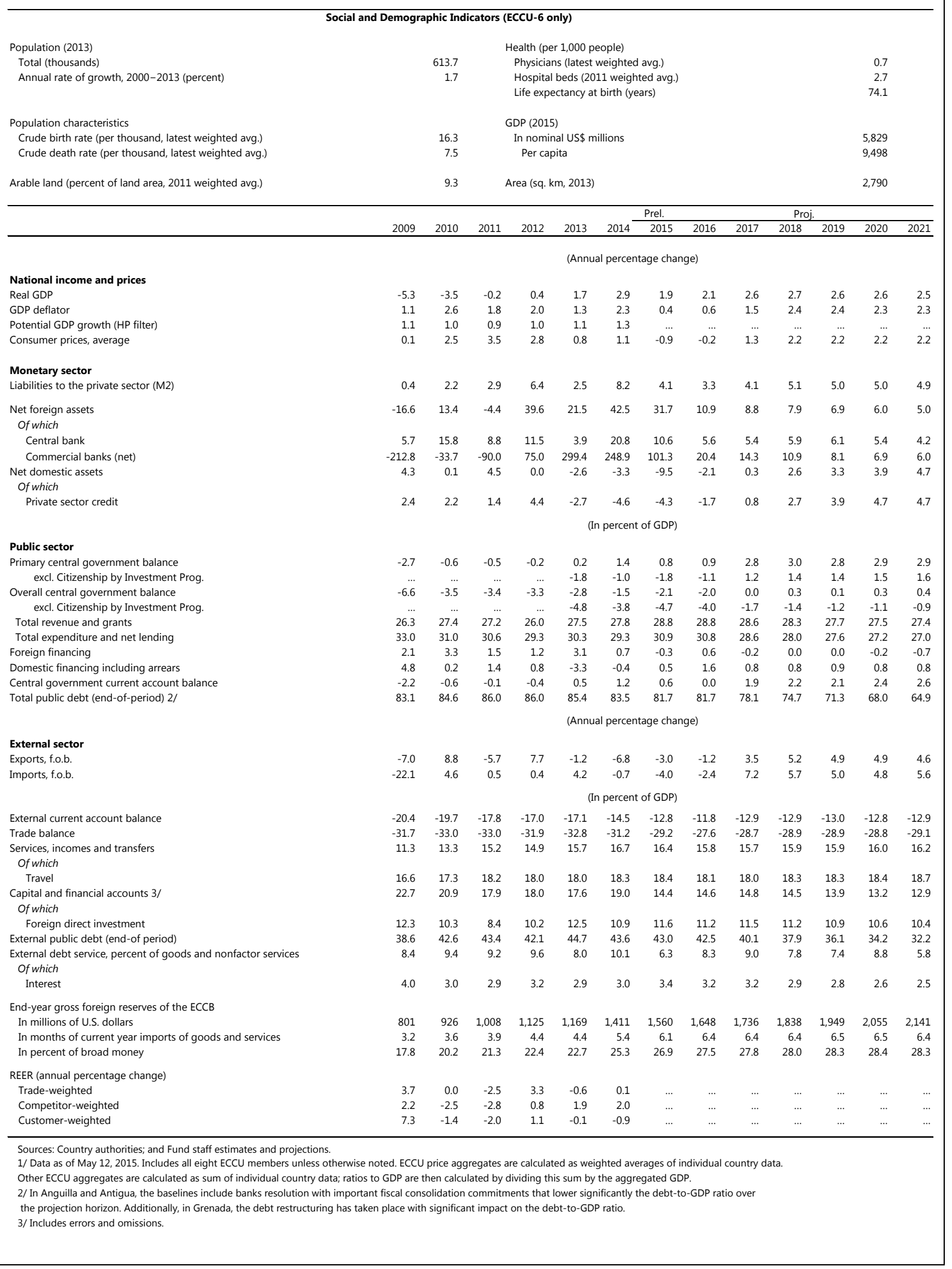




\begin{tabular}{|c|c|c|c|c|c|c|c|c|c|c|c|c|c|}
\hline & & & & & & & $\begin{array}{l}\text { Prel. } \\
\end{array}$ & & & Pro & & & \\
\hline & 2009 & 2010 & 2011 & 2012 & 2013 & 2014 & 2015 & 2016 & 2017 & 2018 & 2019 & 2020 & 2021 \\
\hline \multicolumn{14}{|c|}{ (Annual percentage change) } \\
\hline Real GDP (ECCU-8) & -5.3 & -3.5 & -0.2 & 0.4 & 1.7 & 2.9 & 1.9 & 2.1 & 2.6 & 2.7 & 2.6 & 2.6 & 2.5 \\
\hline Anguilla & -16.5 & -5.8 & -1.2 & -1.8 & 0.3 & 6.7 & 2.2 & 2.2 & 2.5 & 2.7 & 2.8 & 2.7 & 2.7 \\
\hline Antigua and Barbuda & -10.7 & -8.5 & -1.9 & 3.6 & 1.5 & 4.2 & 2.2 & 2.0 & 2.4 & 2.7 & 2.7 & 2.7 & 2.7 \\
\hline Dominica & -0.8 & 0.7 & 1.0 & -1.1 & 1.7 & 3.4 & -3.9 & 1.3 & 2.8 & 2.7 & 2.5 & 2.3 & 1.6 \\
\hline Grenada & -5.6 & -2.0 & 0.1 & -0.6 & 3.2 & 4.8 & 3.1 & 2.3 & 2.7 & 2.7 & 2.7 & 2.7 & 2.7 \\
\hline Montserrat & 0.3 & -3.0 & 6.2 & 1.6 & 2.8 & 1.3 & 0.5 & 1.3 & 1.7 & 2.0 & 2.2 & 2.2 & 2.2 \\
\hline St. Kitts and Nevis & -3.8 & -3.8 & -1.9 & -0.9 & 6.2 & 6.1 & 5.0 & 3.5 & 3.5 & 3.4 & 2.9 & 2.8 & 2.8 \\
\hline St. Lucia & -0.6 & -0.8 & 1.1 & -0.8 & -1.9 & -0.7 & 1.6 & 1.4 & 1.9 & 2.0 & 2.0 & 2.0 & 2.1 \\
\hline St. Vincent and the Grenadines & -2.0 & -2.3 & 0.2 & 1.3 & 2.3 & -0.2 & 1.6 & 2.2 & 3.1 & 3.1 & 3.1 & 3.1 & 3.1 \\
\hline CPI (end of period) & -0.3 & 3.2 & 3.9 & 2.1 & 0.0 & 0.9 & -1.2 & 0.3 & 2.0 & 2.1 & 2.3 & 2.2 & 2.2 \\
\hline Anguilla & -0.8 & 3.8 & 4.4 & -0.3 & 0.8 & -0.9 & -0.1 & 0.8 & 1.7 & 1.9 & 2.3 & 2.3 & 2.3 \\
\hline Antigua and Barbuda & 2.4 & 2.9 & 4.0 & 1.8 & 1.1 & 1.3 & 0.9 & 1.4 & 2.2 & 2.5 & 2.5 & 2.5 & 2.5 \\
\hline Dominica & 3.2 & 0.0 & 1.9 & 1.3 & -0.4 & 0.5 & -0.1 & -0.1 & 1.8 & 1.8 & 2.2 & 2.2 & 2.5 \\
\hline Grenada & -2.3 & 4.2 & 3.5 & 1.8 & -1.2 & -0.6 & -1.2 & -0.2 & 2.8 & 2.3 & 2.2 & 2.1 & 2.1 \\
\hline Montserrat & 2.5 & 2.5 & 4.4 & 3.6 & -0.5 & -1.0 & -0.1 & 0.1 & 0.8 & 1.5 & 2.0 & 2.0 & 2.0 \\
\hline St. Kitts and Nevis & 1.2 & 5.3 & 2.7 & 0.1 & 1.0 & -0.6 & -2.9 & 0.2 & 1.3 & 1.6 & 1.7 & 1.8 & 1.8 \\
\hline St. Lucia & -3.1 & 4.2 & 4.8 & 5.0 & -0.7 & 3.7 & -2.1 & -0.7 & 2.3 & 2.4 & 2.7 & 2.6 & 2.3 \\
\hline St. Vincent and the Grenadines & -2.2 & 0.9 & 4.7 & 1.0 & 0.0 & 0.1 & -2.1 & 1.1 & 1.7 & 1.7 & 1.7 & 1.8 & 1.9 \\
\hline CPI (period average) & 0.1 & 2.5 & 3.5 & 2.8 & 0.8 & 1.1 & -0.9 & -0.2 & 1.3 & 2.2 & 2.2 & 2.2 & 2.2 \\
\hline Anguilla & -0.7 & 2.1 & 4.7 & 1.4 & 0.1 & -0.3 & -0.2 & 1.0 & 1.9 & 2.0 & 2.3 & 2.3 & 2.3 \\
\hline Antigua and Barbuda & -0.6 & 3.4 & 3.5 & 3.4 & 1.1 & 1.1 & 1.0 & 1.4 & 1.8 & 2.4 & 2.5 & 2.5 & 2.5 \\
\hline Dominica & 0.0 & 2.8 & 1.1 & 1.4 & 0.0 & 0.8 & -0.8 & -0.2 & 1.6 & 1.8 & 2.1 & 2.2 & 2.2 \\
\hline Grenada & -0.3 & 3.4 & 3.0 & 2.4 & 0.0 & -1.0 & -1.3 & -0.9 & 2.2 & 2.4 & 2.3 & 2.1 & 2.1 \\
\hline Montserrat & 2.8 & 2.1 & 3.7 & 4.8 & 0.9 & -0.3 & -1.1 & 0.1 & 0.7 & 1.5 & 2.0 & 2.0 & 2.0 \\
\hline St. Kitts and Nevis & 2.1 & 0.7 & 7.1 & 1.4 & 1.0 & 0.7 & -2.8 & -1.3 & 0.8 & 1.5 & 1.7 & 1.7 & 1.8 \\
\hline St. Lucia & -0.2 & 3.3 & 2.8 & 4.2 & 1.5 & 3.5 & -0.7 & -0.8 & 0.2 & 2.8 & 2.6 & 2.6 & 2.3 \\
\hline St. Vincent and the Grenadines & 0.4 & 0.8 & 3.2 & 2.6 & 0.8 & 0.2 & -1.7 & 0.1 & 1.1 & 1.7 & 1.7 & 1.8 & 1.9 \\
\hline Private sector credit & 2.4 & 2.2 & 1.4 & 4.4 & -2.7 & -4.6 & -4.3 & -1.7 & 0.8 & 2.7 & 3.9 & 4.7 & 4.7 \\
\hline Anguilla & 3.1 & 0.2 & 0.1 & -3.4 & -3.5 & -3.8 & -3.9 & -1.7 & 1.2 & 3.0 & 3.8 & 4.0 & 4.0 \\
\hline Antigua and Barbuda & -0.8 & 0.2 & -4.4 & -2.8 & -4.0 & -5.2 & -4.3 & 5.1 & 3.6 & 5.0 & 5.3 & 5.2 & 7.0 \\
\hline Dominica & 6.9 & 9.5 & 6.6 & 4.3 & -0.7 & -2.2 & 0.3 & -3.7 & -0.8 & -0.1 & 1.0 & 1.8 & 1.9 \\
\hline Grenada & 4.5 & 5.6 & 2.2 & 0.2 & -5.7 & -5.1 & -3.8 & 0.1 & 2.6 & 5.4 & 5.2 & 5.1 & 5.1 \\
\hline Montserrat & 15.2 & 16.1 & 10.7 & 9.9 & -11.0 & 0.4 & 5.4 & 8.6 & 5.5 & 5.4 & 5.5 & 5.2 & 5.4 \\
\hline St. Kitts and Nevis & 5.5 & 3.5 & 3.7 & 0.2 & -0.9 & 0.6 & 3.2 & 3.5 & 3.5 & 3.8 & 3.8 & 4.5 & 5.0 \\
\hline St. Lucia & 1.8 & 1.1 & 2.6 & 5.1 & -0.8 & -6.7 & -6.8 & -2.1 & 0.9 & 2.7 & 3.5 & 4.7 & 4.4 \\
\hline St. Vincent and the Grenadines & 1.8 & 1.8 & 4.1 & 3.5 & 1.3 & -0.2 & 2.4 & 3.0 & 4.0 & 4.0 & 4.0 & 4.0 & 4.0 \\
\hline Net credit to public sector & 356.5 & -123.8 & 47.6 & -146.8 & -1721.4 & -71.9 & 164.5 & 5.0 & 1.3 & 4.8 & 2.0 & 2.7 & 2.8 \\
\hline Anguilla & -51.0 & 443.4 & 19.1 & 12.6 & 9.3 & 7.6 & 1.5 & 2.3 & 3.3 & 3.6 & 3.9 & 3.9 & 4.0 \\
\hline Antigua and Barbuda & 154.7 & -16.1 & -7.4 & -26.7 & 42.8 & 13.5 & -12.1 & -20.6 & -11.0 & -11.8 & -13.0 & -15.2 & -24.6 \\
\hline Dominica & -0.6 & -1.1 & -30.6 & 37.9 & -103.5 & 490.0 & -334.0 & -0.2 & 4.6 & 4.7 & 4.8 & 4.6 & 3.7 \\
\hline Grenada & -269.9 & -1661.2 & -17.1 & -152.9 & -194.3 & 239.7 & 128.7 & 6.6 & 6.6 & 6.4 & 6.1 & 2.5 & 2.6 \\
\hline Montserrat & 27.0 & 7.9 & 57.3 & 25.5 & -16.9 & -3.5 & 37.5 & 5.0 & 1.9 & 2.5 & 2.0 & 2.0 & 1.7 \\
\hline St. Kitts and Nevis $1 /$ & 6.7 & 21.9 & -35.8 & -73.4 & -1117.6 & 43.4 & 8.5 & -2.3 & -2.5 & -1.6 & -5.4 & -5.1 & -5.3 \\
\hline St. Lucia & 27.3 & 35.6 & -26.1 & -37.5 & -59.1 & 168.7 & 27.4 & -3.7 & -17.7 & 0.2 & -1.5 & -18.9 & -20.5 \\
\hline St. Vincent and the Grenadines & 17.8 & -82.5 & 99.2 & 11.3 & 23.5 & 44.9 & 25.4 & 16.6 & 38.9 & 32.4 & 14.6 & 13.2 & 13.0 \\
\hline
\end{tabular}


Table 3. ECCU: Selected Central Government Fiscal Indicators by Country, 2009-21 ${ }^{1}$

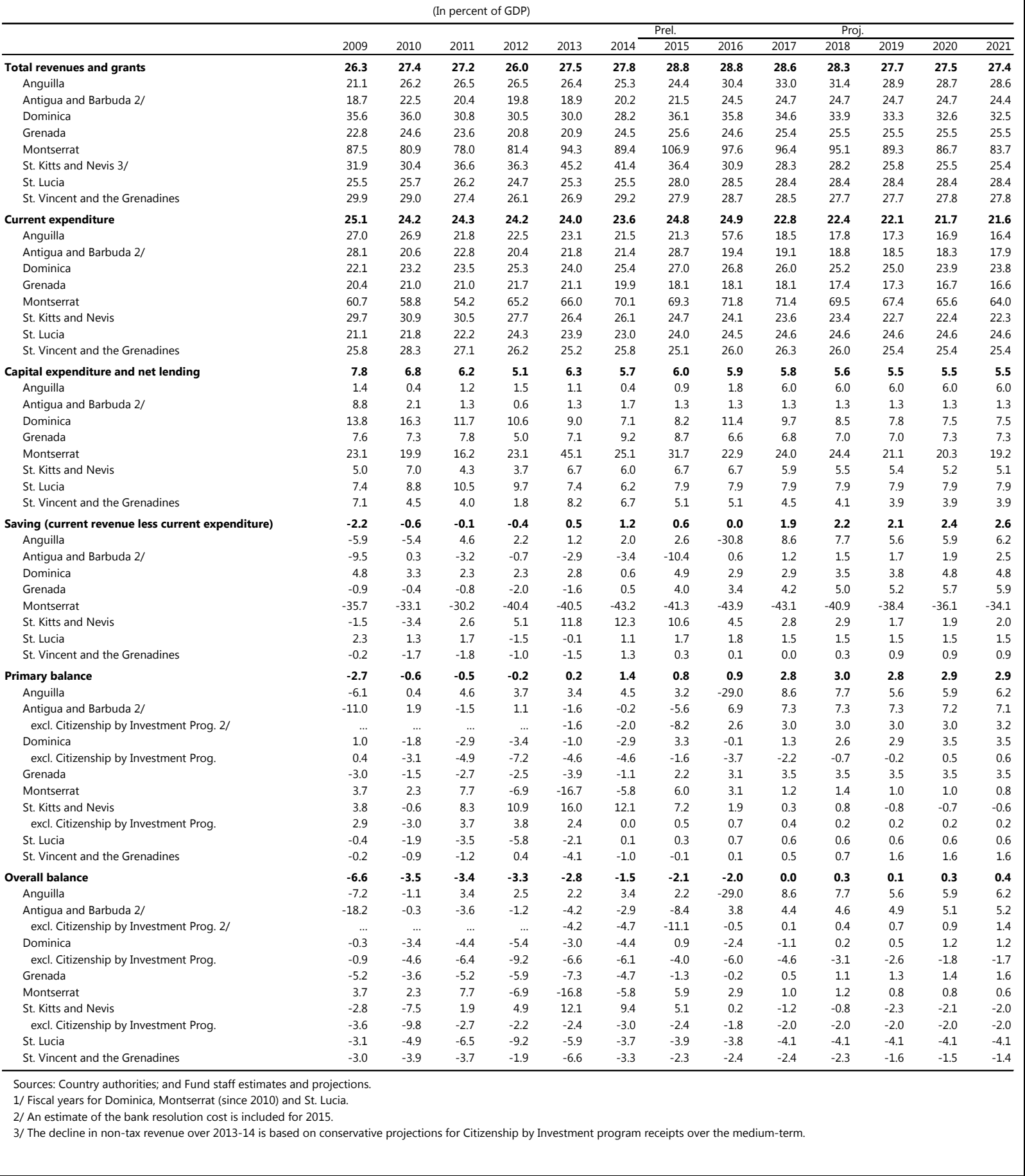




\begin{tabular}{|c|c|c|c|c|c|c|c|c|c|c|c|c|c|}
\hline & & & & & & & Prel. & & & Proj & & & \\
\hline & 2009 & 2010 & 2011 & 2012 & 2013 & $2014^{-}$ & 2015 & 2016 & 2017 & 2018 & 2019 & 2020 & 2021 \\
\hline \multicolumn{14}{|c|}{ (In percent of GDP) } \\
\hline Total public sector debt $2 /$ & 83.1 & 84.6 & 86.0 & 86.0 & 85.4 & 83.5 & 81.7 & 81.7 & 78.1 & 74.7 & 71.3 & 68.0 & 64.9 \\
\hline Anguilla & 25.1 & 32.5 & 28.9 & 30.6 & 30.1 & 24.1 & 22.3 & 54.3 & 47.1 & 40.8 & 36.4 & 31.8 & 27.0 \\
\hline Antigua and Barbuda 3/ & 102.5 & 90.8 & 92.4 & 87.1 & 95.5 & 98.2 & 102.1 & 95.6 & 88.1 & 80.0 & 71.9 & 64.1 & 56.3 \\
\hline Dominica & 62.5 & 66.8 & 69.7 & 72.6 & 74.7 & 83.9 & 85.5 & 87.7 & 87.3 & 85.4 & 83.4 & 81.0 & 79.0 \\
\hline Grenada & 91.1 & 96.9 & 100.7 & 103.3 & 107.6 & 101.4 & 94.3 & 89.2 & 78.8 & 73.2 & 68.3 & 63.6 & 58.9 \\
\hline Montserrat & 5.6 & 5.8 & 4.7 & 4.2 & 4.6 & 4.1 & 7.6 & 7.1 & 6.6 & 6.0 & 5.4 & 4.9 & 4.4 \\
\hline St. Kitts and Nevis & 144.1 & 159.3 & 151.7 & 138.4 & 101.2 & 79.8 & 67.8 & 63.4 & 59.8 & 56.1 & 53.0 & 50.2 & 47.9 \\
\hline St. Lucia & 59.3 & 62.4 & 66.9 & 73.7 & 78.6 & 79.7 & 83.0 & 86.0 & 87.9 & 88.2 & 88.5 & 89.0 & 89.5 \\
\hline St. Vincent and the Grenadines & 63.6 & 65.4 & 68.8 & 72.0 & 74.7 & 79.5 & 73.8 & 76.1 & 78.1 & 78.6 & 77.7 & 76.9 & 76.1 \\
\hline External debt 4/ & 38.6 & 42.6 & 43.4 & 42.1 & 44.7 & 43.6 & 43.0 & 42.5 & 40.1 & 37.9 & 36.1 & 34.2 & 32.2 \\
\hline Anguilla & 6.6 & 24.4 & 21.8 & 22.7 & 22.2 & 20.0 & 18.9 & 23.0 & 20.1 & 17.4 & 14.9 & 12.5 & 10.4 \\
\hline Antigua and Barbuda 3/ & 35.8 & 38.7 & 39.0 & 36.7 & 43.7 & 40.6 & 44.7 & 43.9 & 41.8 & 39.6 & 37.7 & 35.0 & 31.6 \\
\hline Dominica & 43.8 & 49.0 & 49.6 & 52.6 & 52.6 & 53.4 & 56.6 & 56.4 & 55.8 & 53.2 & 51.8 & 50.3 & 49.1 \\
\hline Grenada & 63.9 & 68.9 & 69.0 & 67.6 & 70.2 & 67.1 & 64.1 & 61.9 & 53.5 & 48.8 & 45.0 & 41.3 & 37.7 \\
\hline Montserrat & 5.3 & 5.6 & 4.5 & 4.1 & 3.9 & 3.4 & 6.9 & 6.5 & 6.0 & 5.4 & 4.8 & 4.3 & 3.8 \\
\hline St. Kitts and Nevis & 46.6 & 48.1 & 49.8 & 43.3 & 40.8 & 35.7 & 25.9 & 22.4 & 19.9 & 17.6 & 15.6 & 13.8 & 12.2 \\
\hline St. Lucia & 31.4 & 31.0 & 32.7 & 33.9 & 36.7 & 38.4 & 40.1 & 41.6 & 42.5 & 42.7 & 42.9 & 43.1 & 43.4 \\
\hline St. Vincent and the Grenadines & 32.0 & 40.8 & 43.1 & 40.5 & 42.3 & 45.3 & 43.7 & 43.4 & 41.1 & 38.6 & 36.0 & 33.7 & 31.6 \\
\hline Domestic debt & 44.4 & 42.0 & 42.6 & 43.9 & 40.7 & 39.9 & 38.7 & 39.2 & 38.1 & 36.7 & 35.2 & 33.9 & 32.7 \\
\hline Anguilla & 18.6 & 8.1 & 7.1 & 7.9 & 7.9 & 4.1 & 3.4 & 31.3 & 27.0 & 23.3 & 21.5 & 19.3 & 16.7 \\
\hline Antigua and Barbuda 3/ & 66.7 & 52.0 & 53.4 & 50.5 & 51.8 & 57.6 & 57.3 & 51.7 & 46.3 & 40.4 & 34.3 & 29.1 & 24.7 \\
\hline Dominica & 18.7 & 17.8 & 20.1 & 20.0 & 22.1 & 30.5 & 28.9 & 31.3 & 31.5 & 32.3 & 31.6 & 30.7 & 29.9 \\
\hline Grenada & 27.2 & 28.0 & 31.7 & 35.8 & 37.4 & 34.3 & 30.2 & 27.3 & 25.3 & 24.4 & 23.3 & 22.3 & 21.2 \\
\hline Montserrat & 0.3 & 0.3 & 0.2 & 0.1 & 0.7 & 0.7 & 0.7 & 0.7 & 0.7 & 0.6 & 0.6 & 0.6 & 0.6 \\
\hline St. Kitts and Nevis & 97.5 & 111.2 & 101.9 & 95.0 & 60.4 & 44.1 & 41.8 & 40.9 & 39.9 & 38.4 & 37.3 & 36.3 & 35.8 \\
\hline St. Lucia & 27.9 & 31.3 & 34.2 & 39.8 & 41.9 & 41.2 & 42.9 & 44.4 & 45.3 & 45.5 & 45.7 & 45.9 & 46.1 \\
\hline \multirow[t]{2}{*}{ St. Vincent and the Grenadines } & 31.5 & 24.7 & 25.7 & 31.5 & 32.4 & 34.2 & 30.1 & 32.8 & 37.0 & 40.0 & 41.7 & 43.2 & 44.5 \\
\hline \multirow{2}{*}{\multicolumn{14}{|c|}{ Implied interest rate on central government debt }} \\
\hline & & & & & & & & & & & & & \\
\hline Anguilla & 4.9 & 5.1 & 4.3 & 4.1 & 4.2 & 5.0 & 4.8 & 0.0 & 0.0 & 0.0 & 0.0 & 0.0 & 0.0 \\
\hline Antigua and Barbuda 3/ & 8.5 & 2.8 & 2.7 & 3.1 & 3.4 & 3.3 & 3.3 & 3.9 & 4.0 & 4.0 & 4.1 & 4.3 & 4.4 \\
\hline Dominica & 2.6 & 2.8 & 2.6 & 3.3 & 3.2 & 2.4 & 3.7 & 3.5 & 3.7 & 3.8 & 3.8 & 3.9 & 4.0 \\
\hline Grenada & 2.6 & 2.4 & 2.6 & 3.5 & 3.3 & 3.7 & 3.9 & 3.8 & 3.9 & 3.4 & 3.4 & 3.4 & 3.4 \\
\hline Montserrat & 4.2 & 4.6 & 1.7 & 1.4 & 1.2 & 1.2 & 2.6 & 3.9 & 3.7 & 4.4 & 4.5 & 4.7 & 5.0 \\
\hline St. Kitts and Nevis & 5.9 & 5.5 & 5.3 & 5.4 & 4.9 & 4.0 & 3.8 & 3.3 & 3.2 & 3.3 & 3.4 & 3.5 & 3.6 \\
\hline St. Lucia & 5.1 & 5.3 & 4.9 & 5.0 & 5.3 & 5.1 & 5.4 & 5.6 & 5.6 & 5.6 & 5.6 & 5.6 & 5.6 \\
\hline St. Vincent and the Grenadines & 5.3 & 5.3 & 4.0 & 3.9 & 3.9 & 3.4 & 3.3 & 3.5 & 3.9 & 4.3 & 4.5 & 4.6 & 4.6 \\
\hline \multicolumn{14}{|c|}{ Implied interest rates on central government external debt } \\
\hline Anguilla & 5.4 & 1.4 & 4.1 & 3.7 & 3.8 & 4.0 & 3.9 & 0.0 & 0.0 & 0.0 & 0.0 & 0.0 & 0.0 \\
\hline Antigua and Barbuda 3/5/ & 7.4 & 1.5 & 2.2 & 1.4 & 2.3 & 2.5 & 2.4 & 2.2 & 2.7 & 2.8 & 3.0 & 3.1 & 3.4 \\
\hline Dominica & 2.0 & 3.0 & 1.7 & 2.8 & 2.4 & 1.9 & 2.5 & 2.3 & 2.3 & 2.3 & 2.2 & 2.2 & 2.4 \\
\hline Grenada & 2.0 & 1.8 & 1.9 & 2.9 & 2.5 & 3.0 & 3.8 & 3.3 & 3.5 & 2.9 & 2.8 & 2.7 & 2.8 \\
\hline Montserrat & 2.4 & 1.6 & 2.1 & 1.6 & 2.1 & 2.1 & 2.6 & 2.1 & 1.7 & 2.2 & 2.1 & 2.1 & 2.2 \\
\hline St. Kitts and Nevis & 6.1 & 5.6 & 4.2 & 5.1 & 1.4 & 2.1 & 2.7 & 2.2 & 2.1 & 2.1 & 2.0 & 2.0 & 2.0 \\
\hline St. Lucia & 4.5 & 4.5 & 3.8 & 4.2 & 4.3 & 3.9 & 4.2 & 4.3 & 4.3 & 4.3 & 4.3 & 4.3 & 4.3 \\
\hline St. Vincent and the Grenadines 6/ & 4.0 & 3.1 & 3.0 & 3.2 & 2.6 & 2.3 & 2.2 & 2.5 & 2.6 & 2.6 & 2.7 & 2.7 & 2.7 \\
\hline \multicolumn{14}{|c|}{ Implied interest rates on central government domestic debt } \\
\hline Anguilla & 4.8 & $\ldots$ & 4.8 & 5.2 & 5.2 & 9.3 & 9.3 & 0.0 & 0.0 & 0.0 & 0.0 & 0.0 & 0.0 \\
\hline Antigua and Barbuda 3/5/ & 9.1 & 3.8 & 3.0 & 4.5 & 4.3 & 3.9 & 3.9 & 5.2 & 5.2 & 5.2 & 5.3 & 5.6 & 5.6 \\
\hline Dominica & 3.9 & 2.4 & 5.0 & 4.7 & 5.5 & 3.5 & 6.8 & 6.7 & 7.3 & 7.5 & 8.0 & 8.2 & 8.2 \\
\hline Grenada & 4.2 & 4.1 & 4.2 & 4.8 & 4.8 & 5.1 & 4.0 & 5.1 & 4.8 & 4.5 & 4.6 & 4.7 & 4.7 \\
\hline Montserrat & 10.4 & 19.0 & $\ldots$ & $\ldots$ & $\ldots$ & 0.0 & 2.5 & 15.4 & 16.8 & 18.2 & 19.6 & 20.9 & 22.1 \\
\hline St. Kitts and Nevis & 5.8 & 5.4 & 5.9 & 5.6 & 8.2 & 5.9 & 4.7 & 4.0 & 3.9 & 4.1 & 4.2 & 4.3 & 4.3 \\
\hline St. Lucia & 5.8 & 6.1 & 6.0 & 5.7 & 6.4 & 6.3 & 6.7 & 6.9 & 6.9 & 6.9 & 6.9 & 6.9 & 6.9 \\
\hline St. Vincent and the Grenadines 6/ & 7.0 & 9.3 & 6.3 & 4.7 & 5.9 & 5.1 & 5.1 & 5.3 & 6.0 & 6.6 & 6.9 & 6.9 & 6.9 \\
\hline \multicolumn{14}{|c|}{ Sources: Country authorities; and Fund staff estimates and projections. } \\
\hline \multicolumn{14}{|c|}{ 1/ Fiscal years for Dominica, Montserrat (since 2010) and St. Lucia. } \\
\hline \multirow{2}{*}{\multicolumn{14}{|c|}{$\begin{array}{l}\text { 2/ In Anguilla and Antigua, the baselines include banks resolution with important fiscal consolidation commitments that lower significantly the debt-to-GDP } \\
\text { ratio over the projection horizon. Additionally, in Grenada, the debt restructuring has taken place with significant impact on the debt-to-GDP ratio. }\end{array}$}} \\
\hline & & & & & & e debt-to & SDP ratio & & & & & & \\
\hline \multicolumn{14}{|c|}{ 3/ An estimate of the bank resolution cost is included for 2015 . } \\
\hline 4/ Includes external arrears. & & & & & & & & & & & & & \\
\hline 5/ Interest payments from 2009 are $o$ & & & & & & & & & & & & & \\
\hline $\begin{array}{l}\text { 6/ The increase (decrease) in implicit } \\
\text { external borrowing, resulting in a lars }\end{array}$ & 010 is du & $\begin{array}{l}\text { to the pr } \\
\text { ernal) det }\end{array}$ & $\begin{array}{l}\text { jected rep } \\
\text { outstandi }\end{array}$ & yment of & omestic & bt fina & & & & & & & \\
\hline
\end{tabular}


Table 5. ECCU: Monetary Survey, 2009-18

\begin{tabular}{|c|c|c|c|c|c|c|c|c|c|c|}
\hline & \multirow[b]{2}{*}{2009} & \multirow[b]{2}{*}{2010} & \multirow[b]{2}{*}{2011} & \multirow[b]{2}{*}{2012} & \multirow[b]{2}{*}{2013} & \multirow[b]{2}{*}{2014} & \multirow[b]{2}{*}{$2015^{-}$} & \multicolumn{3}{|c|}{ Proj. } \\
\hline & & & & & & & & 2016 & 2017 & 2018 \\
\hline \multicolumn{11}{|c|}{ (In millions of EC dollars, end of period) } \\
\hline Net foreign assets & 1,895 & 2,148 & 2,053 & 2,865 & 3,481 & 4,960 & 6,533 & 7,245 & 7,884 & 8,508 \\
\hline Central bank & 2,157 & 2,498 & 2,718 & 3,031 & 3,150 & 3,804 & 4,206 & 4,443 & 4,681 & 4,957 \\
\hline Commercial banks (net) & -262 & -350 & -665 & -166 & 331 & 1,156 & 2,327 & 2,801 & 3,203 & 3,551 \\
\hline Assets & 4,015 & 3,200 & 3,864 & 4,045 & 4,206 & 4,940 & $\ldots$ & $\ldots$ & $\ldots$ & $\ldots$ \\
\hline Liabilities & $-4,277$ & $-3,550$ & $-4,529$ & $-4,212$ & $-3,874$ & $-3,784$ & $\ldots$ & $\ldots$ & $\ldots$ & $\ldots$ \\
\hline Net domestic assets & 10,238 & 10,252 & 10,709 & 10,709 & 10,429 & 10,085 & 9,125 & 8,934 & 8,961 & 9,190 \\
\hline Public sector credit, net & 382 & -91 & -134 & 63 & $-1,017$ & -286 & -755 & -793 & -804 & -842 \\
\hline Central government & 1,646 & 1,189 & 1,262 & 1,576 & 619 & 1,608 & 1,247 & 1,267 & 1,342 & 1,412 \\
\hline Other public sector & $-1,264$ & $-1,280$ & $-1,396$ & $-1,513$ & $-1,621$ & $-1,894$ & $-2,003$ & $-2,061$ & $-2,146$ & $-2,254$ \\
\hline Private sector credit, net & 12,325 & 12,601 & 12,773 & 13,334 & 12,980 & 12,377 & 11,841 & 11,636 & 11,727 & 12,038 \\
\hline (real terms) & 9,093 & 9,067 & 8,877 & 9,014 & 8,704 & 8,212 & 7,857 & 7,789 & 7,865 & 7,972 \\
\hline Other items (net) & $-2,469$ & $-2,258$ & $-1,930$ & $-2,688$ & $-1,195$ & $-2,006$ & $-1,960$ & $-1,909$ & $-1,963$ & $-2,006$ \\
\hline Broad money (M2) & 12,133 & 12,400 & 12,762 & 13,574 & 13,910 & 15,045 & 15,658 & 16,178 & 16,844 & 17,698 \\
\hline Money & 2,462 & 2,497 & 2,618 & 2,804 & 2,810 & 3,173 & 3,461 & 3,562 & 3,708 & 3,896 \\
\hline Currency in circulation & 836 & 834 & 876 & 896 & 926 & 991 & $\ldots$ & $\ldots$ & $\ldots$ & $\ldots$ \\
\hline Demand deposits & 1,841 & 1,881 & 1,984 & 2,170 & 2,188 & 2,457 & $\ldots$ & $\ldots$ & $\ldots$ & $\ldots$ \\
\hline Quasi-money & 9,671 & 9,903 & 10,143 & 10,770 & 11,312 & 11,872 & 12,197 & 12,617 & 13,136 & 13,802 \\
\hline Time deposits & 2,705 & 2,869 & 2,983 & 3,026 & 2,904 & 2,732 & $\ldots$ & $\ldots$ & $\ldots$ & $\ldots$ \\
\hline Savings deposits & 5,222 & 5,282 & 5,484 & 5,969 & 6,400 & 6,787 & $\ldots$ & $\ldots$ & $\ldots$ & $\ldots$ \\
\hline Foreign currency deposits & 1,743 & 1,752 & 1,677 & 1,775 & 2,008 & 2,354 & $\ldots$ & $\ldots$ & $\ldots$ & $\ldots$ \\
\hline \multicolumn{11}{|c|}{ (Annual percentage change) } \\
\hline Net foreign assets & -16.6 & 13.4 & -4.4 & 39.6 & 21.5 & 42.5 & 31.7 & 10.9 & 8.8 & 7.9 \\
\hline Net domestic assets, o.w. & 4.3 & 0.1 & 4.5 & 0.0 & -2.6 & -3.3 & -9.5 & -2.1 & 0.3 & 2.6 \\
\hline Public sector credit, net & 356.5 & -123.8 & 47.6 & -146.8 & $-1,721.4$ & -71.9 & 164.5 & 5.0 & 1.3 & 4.8 \\
\hline Private sector credit, net & 2.4 & 2.2 & 1.4 & 4.4 & -2.7 & -4.6 & -4.3 & -1.7 & 0.8 & 2.7 \\
\hline Broad money (M2) & 0.4 & 2.2 & 2.9 & 6.4 & 2.5 & 8.2 & 4.1 & 3.3 & 4.1 & 5.1 \\
\hline NFA contribution & -3.1 & 2.1 & -0.8 & 6.4 & 4.5 & 10.6 & 10.5 & 4.5 & 4.0 & 3.7 \\
\hline NDA contribution & 3.5 & 0.1 & 3.7 & 0.0 & -2.1 & -2.5 & -6.4 & -1.2 & 0.2 & 1.4 \\
\hline Money & -6.8 & 1.4 & 4.8 & 7.1 & 0.2 & 12.9 & 9.1 & 2.9 & 4.1 & 5.1 \\
\hline NFA contribution & 4.4 & 13.9 & 8.8 & 12.0 & 4.2 & 23.3 & 12.7 & 6.9 & 6.7 & 7.4 \\
\hline NDA contribution & -11.2 & -12.4 & -3.9 & -4.9 & -4.0 & -10.4 & -3.6 & -4.0 & -2.6 & -2.4 \\
\hline Quasi-money & 2.4 & 2.4 & 2.4 & 6.2 & 5.0 & 5.0 & 2.7 & 3.4 & 4.1 & 5.1 \\
\hline \multicolumn{11}{|c|}{ (In percent of GDP) } \\
\hline Net foreign assets & 13.0 & 14.9 & 13.9 & 19.0 & 22.5 & 30.3 & 39.9 & 43.2 & 45.7 & 47.4 \\
\hline Net domestic assets, o.w. & 70.5 & 71.0 & 72.6 & 71.2 & 67.5 & 61.7 & 55.8 & 53.3 & 51.9 & 51.2 \\
\hline Public sector credit, net & 2.6 & -0.6 & -0.9 & 0.4 & -6.6 & -1.7 & -4.6 & -4.7 & -4.7 & -4.7 \\
\hline Private sector credit, net & 84.9 & 87.3 & 86.6 & 88.6 & 84.0 & 75.7 & 72.4 & 69.4 & 68.0 & 67.0 \\
\hline Broad money (M2) & 83.5 & 85.9 & 86.5 & 90.2 & 90.0 & 92.0 & 95.7 & 96.5 & 97.6 & 98.5 \\
\hline Money & 17.0 & 17.3 & 17.7 & 18.6 & 18.2 & 19.4 & 21.2 & 21.2 & 21.5 & 21.7 \\
\hline Quasi-money & 66.6 & 68.6 & 68.8 & 71.6 & 73.2 & 72.6 & 74.6 & 75.3 & 76.2 & 76.8 \\
\hline \multicolumn{11}{|l|}{ Interest rates (percent per year) } \\
\hline ECCB policy rate & 6.50 & 6.50 & 6.50 & 6.50 & 6.50 & 6.50 & 6.50 & $\ldots$ & $\ldots$ & $\ldots$ \\
\hline US policy rate & 0.125 & 0.125 & 0.125 & 0.125 & 0.125 & 0.125 & 0.136 & $\ldots$ & $\ldots$ & $\ldots$ \\
\hline Interbank market rate & 6.16 & 6.22 & 4.88 & 6.31 & 6.45 & 6.03 & 0.00 & $\ldots$ & $\ldots$ & $\ldots$ \\
\hline Time deposit rate & 4.58 & 4.51 & 4.17 & 4.34 & 4.06 & 3.60 & 2.95 & $\ldots$ & $\ldots$ & $\ldots$ \\
\hline Demand deposit rate & 0.69 & 0.74 & 0.68 & 0.74 & 0.58 & 0.48 & 0.37 & $\ldots$ & $\ldots$ & $\ldots$ \\
\hline Weighted average lending rate & 9.49 & 9.48 & 9.53 & 8.91 & 8.94 & 8.92 & 8.74 & $\ldots$ & $\ldots$ & $\ldots$ \\
\hline
\end{tabular}




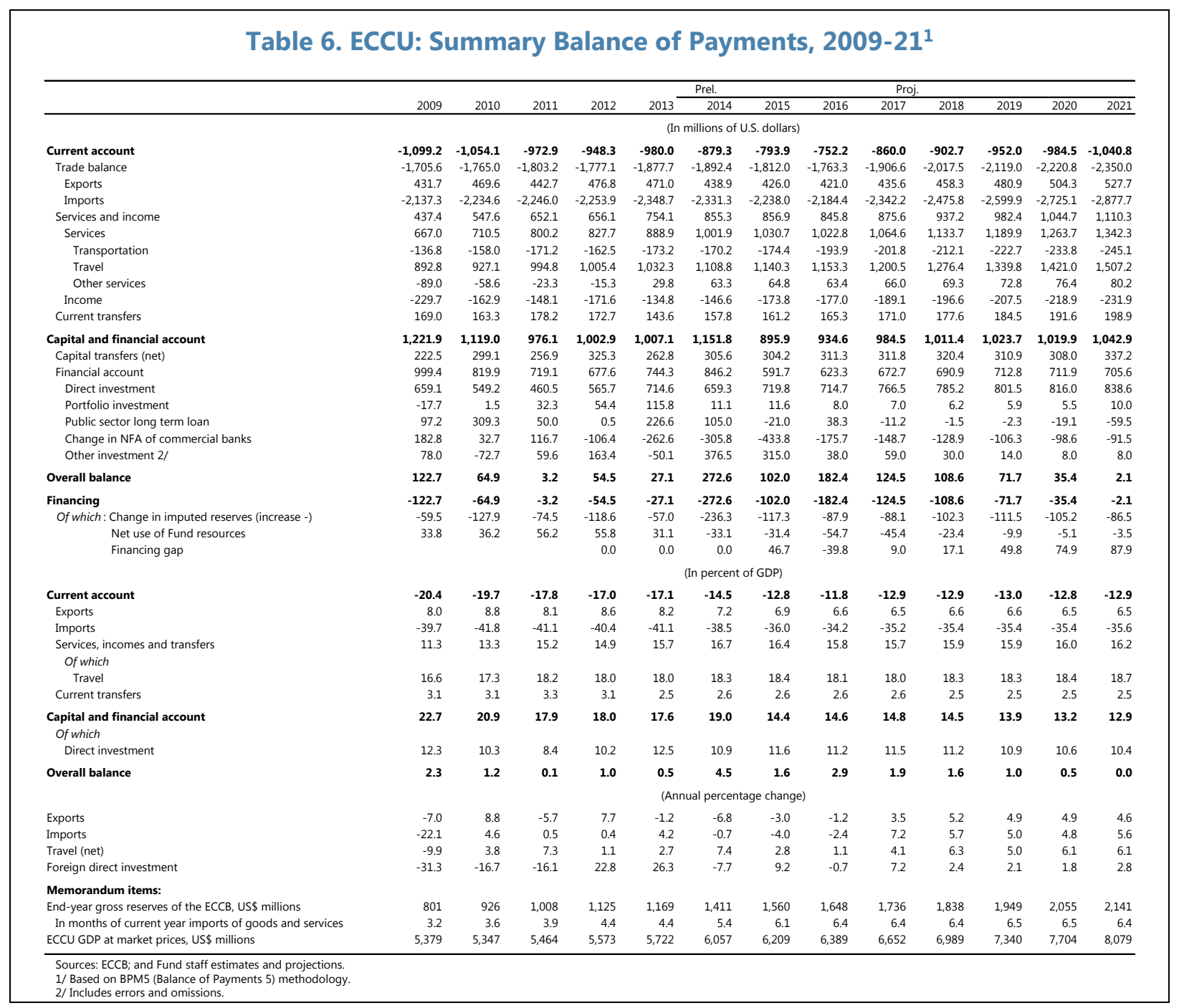




\section{Table 7. Recent Fund Staff Regional Consultation Recommendations}

Proposed Action Status of Implementation

\section{Banking}

Complete bank diagnostics, incl. asset quality review

AQR of all indigenous banks and selected foreign banks was completed in 2015

(AQR) to assess banks' capital shortfalls, and a viability assessment (dynamic modeling) of banks' business results of the assessment communicated to the banks. Dynamic modeling

models. exercise completed in 2015

Resolve three banks under conservatorship.

Bank in Antigua and Barbuda was resolved in November, 2015; two banks in Anguilla were resolved in April 2016.

Approve regional banking legislation.

The new Banking Act and the Eastern Caribbean Asset Management Company (ECAMC) approved in all jurisdictions. ECAMC is yet to become operational. New regional foreclosure legislation in drafting stage. Drafting of credit reporting bill completed, currently undergoing review by the financial industry.

\section{Monetary}

Remove/lower minimum saving deposit rate (MSR).

MSR was lowered from 3 to 2 percent, effective May 2015. Discussions continue for potential further reduction.

Fiscal

Adopt fiscal responsibility legislation.

Adopted by Grenada.

Adopt medium-term fiscal framework consistent with

Mixed progress. Some countries have advanced in this area.

reaching the debt target of 60 percent.

Strengthen CIP governance and improve program

Efforts to strengthen regional CIP programs are ongoing.

transparency

\section{External competitiveness}

Implement structural reforms to reduce costs and

improve the business environment.

National energy policies have been drawn up by most jursidictions. Efforts to increase reliance on renewable energy resources and improve competitiveness continue, including the implementation of a single financial and customs space. 


\section{Annex I. Citizenship-by-Investment Programs in ECCU1}

\section{Citizenship-by-Investment Programs (CIPs) have proliferated across the region in recent years, driven by their growing appeal as a financing mechanism for the public and private sector in host jurisdictions, together with increased demand for global mobility. The} experience of St. Kitts and Nevis, one of the oldest of such programs in the World, has been instrumental. The significant revenue windfall to the government and the substantial influx of foreign investments through the program propelled the country's strong macroeconomic recovery and buoyant fiscal performance in recent years. This spurred the interest of neighboring islands facing persistent economic challenges in the aftermath of the global financial crisis in adopting similar schemes. As a result, three ECCU

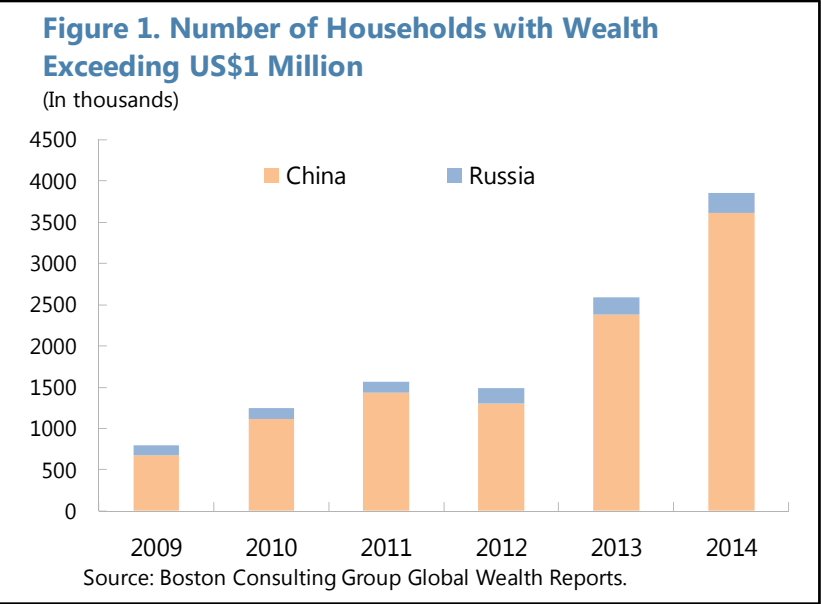
countries, Antigua and Barbuda, Grenada and St. Lucia, launched their own CIPs over 2013-2015.2 On the demand side, the surge of inflows through CIPs is largely explained by the significant rise in the number of high net-worth individuals, largely from emerging economies. These persons that are typically interested in acquiring an alternative travel document to facilitate international travel by easing visa restrictions, and/or secure a potential safe haven from possible or existing economic and political turmoil in their home countries. Other considerations include estate and tax planning. Tentative data from active CIPs indicate that the bulk of demand originates from China and the Middle East, and to a lesser extent, Russia and some advanced countries.

\section{The launch of new CIPs has increased competition within a relatively homogenous} market, raising risks to the long-term sustainability of inflows. The structure of CIPs in the region is fairly similar, featuring at least two investment options: i) a lump sum contribution to a National Development Fund (NDF); or ii) redeemable investment in the private sector, typically in real estate or a business venture; in addition to some government fee (see Table 1 ). ${ }^{3}$ Moreover, as of 2016, citizens of Dominica, Grenada and St. Lucia became eligible for visa-free travel to the Schengen area, diminishing privileges that used to differentiate CIPs of Antigua and Barbuda and St.

\footnotetext{
${ }^{1}$ Prepared by Ahmed El Ashram.

2 Programs administered by St. Kitts and Nevis and Dominica date back to 1984 and 1993, respectively. In 2013, Antigua and Barbuda launched its own CIP, while Grenada revived its previously retired scheme. In early 2016, St. Lucia became the fifth ECCU country to launch a CIP.

${ }^{3}$ St. Lucia's newly launched CIP features a fourth option that involves investment in government securities for a period of 5 years, similar to some immigrant investment programs in the US, the UK and, formerly, the Canadian Federal Government.
} 
Kitts and Nevis. ${ }^{4}$ In this context, price competition among programs will increase risks to future inflows, particularly for more established programs like St. Kitts and Nevis, which has already been adversely impacted by the imposition of visa requirements by Canada in November 2014, and the FinCen Advisory in May 2014. Despite the lower investment requirements in Dominica, the number of applicants needed to generate 1 percent of GDP in inflows is the lowest among all five programs, given the small size of its economy, creating incentives to continue competing on price terms. ${ }^{5}$ The appeal of different CIPs also depends on processing speed and restrictions on the applicants' country of origin. ${ }^{6}$ Finally, competition from outside the region, particularly from Malta's newly launched CIP and other residency-by-investment schemes in Europe that offer similar visa-free access to the EU, have further increased downside risks to future inflows to Caribbean programs. ${ }^{7}$
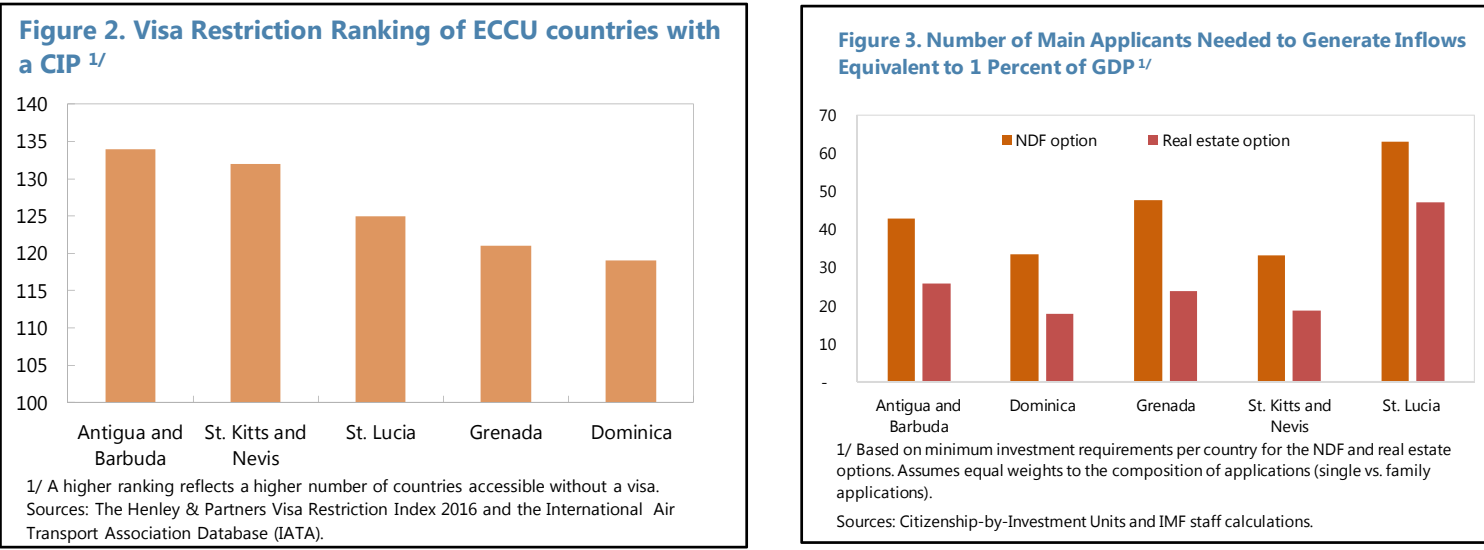

\section{Developments in future CIP inflows have critical macroeconomic implications for the ECCU macroeconomic outlook; to the extent they already underpin growth and fiscal}

performance. Because CIP inflows are potentially volatile, increased reliance on these inflows to finance the budget would trigger painful fiscal adjustment if they diminish or face a sudden stop,

\footnotetext{
${ }^{4}$ On May $28^{\text {th }}$, the EU signed visa-waiver agreements with five Caribbean countries, including Dominica, Grenada and St. Lucia, which became effective in 2016. Antigua and Barbuda and St. Kitts and Nevis were granted visa waiver status by the EU in 2009. In November 2014, Canadian authorities reintroduced visa requirements for citizens of St. Kitts and Nevis.

${ }^{5}$ Dominica is expected to revise its CIP investment requirements upwards in $2 \mathrm{H} 2016$.

${ }^{6}$ St. Kitts and Nevis' CIP does not accept applications from nationals of Iran and Afghanistan, irrespective of their place of domicile. Antigua and Barbuda imposed similar restrictions on nationals of Afghanistan, Iran, Iraq, North Korea, Somalia, and Yemen, except those who are lawful permanent residents in the US, the UK or Canada. In March 2016, Antigua and Barbuda eased this restriction by accepting applications from Iranian and Iraqi nationals, irrespective of their place of domicile.

${ }^{7}$ Both Malta and Cyprus administer citizenship-by-programs that are quite similar to Caribbean CIPs, despite significantly higher investment requirements. Malta's CIP has been particularly attractive to high net-worth applicants given Malta's EU membership status. Further, since 2012, a number of other EU member states, including France, Greece, Hungary, Ireland, Portugal and Spain, launched residency-by-investment programs with privileges of visafree travel, the right to reside, work and establish businesses across the EU.
} 
while a cessation of CIP-related private investments can generate boom-bust cycles in the domestic economy. The risk is most acute in St. Kitts and Nevis, where inflows have been most significant, reaching an estimated cumulative 45 percent of GDP over 2013-2015, with inflows to the budget alone reaching a peak of 14 percent of GDP in 2014. The newly launched program by Antigua and Barbuda has already attracted significant inflows to the public sector, in excess of an estimated $71 / 2$ percent of GDP in $2015,{ }^{8}$

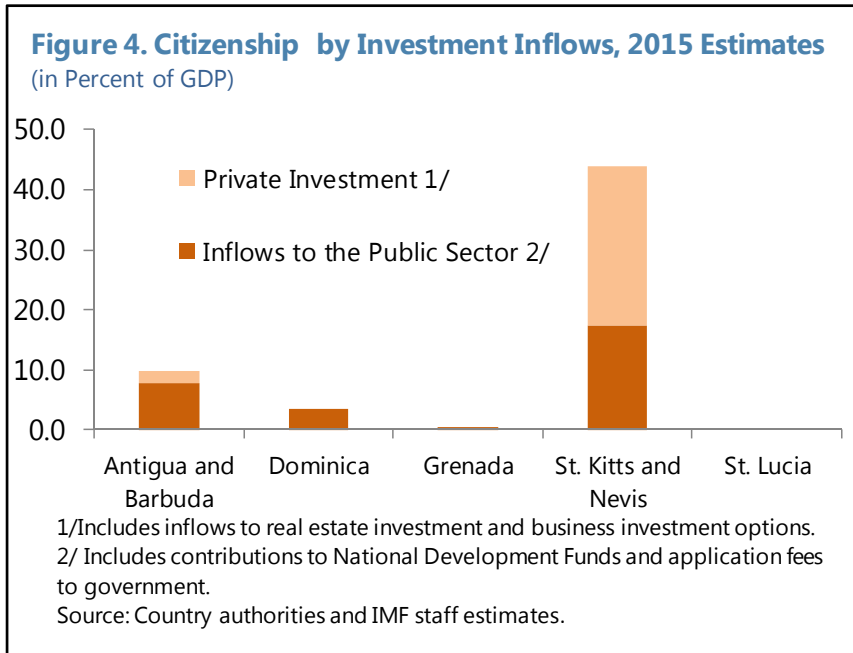

and has proven critical to financing the fiscal deficit and balancing elevated risks of debt distress. Dominica is also becoming increasingly reliant on these inflows to finance the budget. A sudden stop scenario would also result in a sharp deterioration in the external position of countries with large CIP inflows.

\section{Prudent management of CIP inflows is critical to ensuring medium-term fiscal} sustainability in the ECCU. A recent IMF working paper by Xu et al (2015) proposed a framework to prudently manage volatile CIP inflows, primarily through saving the bulk of inflows to the public sector and regulating the pace of private sector investments, where inflows are large. ${ }^{9}$ The framework seeks to prevent the buildup of unsustainable dependency, particularly with respect to the financing of recurrent expenditure, and hence mitigate risks of painful adjustment in the event of a sudden stop. This is achieved through anchoring the fiscal framework with a sustainable underlying primary balance target, excluding CIP revenues.

\section{A broader fiscal perimeter, reflecting all CIP-related revenues and expenditures, is needed to properly analyze the true fiscal policy stance in ECCU countries with CIPs. In} particular, all CIP revenues to the public sector, and related expenditure, should be recorded on budget. In St. Kitts and Nevis, contributions to the Sugar Industry Diversification Foundation (SIDF)the country's NDF-are not reported on budget, while in Antigua and Barbuda, only NDF resources are managed on budget with the CIP Unit surplus from application fees to government are managed at Cabinet's discretion, outside the budget framework. ${ }^{10}$ Similar information gaps exist in Dominica. Funding fiscal or quasi-fiscal operations from outside the budget weakens the assessment

\footnotetext{
${ }^{8}$ Includes total contributions to the National Development Fund in 2015, and updated staff estimates of government processing fees that are not reported on budget.

${ }^{9}$ See IMF Working Paper 15/193: "Too Much of a Good Thing? Prudent Management of Inflows under Economic Citizenship Programs".

${ }^{10}$ In St. Kitts and Nevis, efforts are ongoing to improve transparency of the SIDF financial reporting and integrate it with the government's consolidated fund. In Antigua and Barbuda, the authorities have agreed to implement a mechanism to transfer a share of the CIP Unit surplus to the consolidated central government account as part of the proposed fiscal consolidation program in 2016.
} 
of the underlying fiscal stance and provides incomplete information on the actual size of governments' spending commitments. Staff continues to press for comprehensive and consistent reporting of CIP revenues in the external and fiscal accounts of the ECCU and for the monitoring of the underlying fiscal position, net of CIP revenues to the public sector.

\section{Recent policy guidance on using CIP inflows by Fund staff has focused on enhancing} macroeconomic resilience across the region. Staff continues to urge authorities to use CIP revenue windfalls to the public sector to reduce the public debt load, build precautionary buffers and support strategic public investment projects in their economies, where appropriate. In particular, staff recommends accumulating a share of these resources in a fund to address costly natural disaster shocks and finance disaster resilient infrastructure. Funding capital projects using CIP resources should ensure rigorous project evaluation and selection and that sufficient resources have been secured in advance to finance projects to completion, to limit potential contingent liability to government.

\section{A comprehensive governance framework is crucial to mitigating increased risks facing}

CIPs. Rising global migration pressures, elevated security concerns and geopolitical tensions may trigger adverse actions by the international community, in particular the suspension of visa-free travel for citizens of CIP countries. The authorities should thus ensure that all necessary safeguards are strictly maintained, including a rigorous due diligence process for applicants, a full-fledged AML/CFT framework, and regulations to ensure the transparency and exchange of tax information. More generally, the number of citizenships granted, revenues earned, and their use, including the amounts saved, spent or invested, should be made public with the budget documents and be subject to financial audits. 
Table 1. Investment Requirements of Citizenship-by-Investment Programs in the Caribbean $1 /$ (In US dollars)

\begin{tabular}{|c|c|c|c|c|c|c|c|c|c|}
\hline \multirow{3}{*}{$\begin{array}{c}\begin{array}{c}\text { Contributions and } \\
\text { Investments }\end{array} \\
\text { Type of Application }\end{array}$} & \multicolumn{5}{|c|}{ Option I } & \multicolumn{4}{|c|}{ Option II } \\
\hline & \multicolumn{2}{|c|}{ Government Fee } & \multirow[t]{2}{*}{$\&$} & \multicolumn{2}{|c|}{$\begin{array}{c}\text { Contribution to } \\
\text { National Development } \\
\text { Fund (NDF) }\end{array}$} & \multicolumn{2}{|c|}{ Government Fee } & \multicolumn{2}{|c|}{$\begin{array}{c}\text { Redeemable } \\
\text { Investment 6/ }\end{array}$} \\
\hline & $\begin{array}{c}\text { Single } \\
\text { Appplicant }\end{array}$ & Family & & $\begin{array}{c}\text { Single } \\
\text { Appplicant }\end{array}$ & Family & $\begin{array}{c}\text { Single } \\
\text { Appplicant }\end{array}$ & Family & $\begin{array}{c}\text { Single } \\
\text { Appplicant }\end{array}$ & Family \\
\hline Antigua and Barbuda 2/ & 50,000 & 150,000 & & 200,000 & 200,000 & 50,000 & 150,000 & $\begin{array}{c}\text { Real estate: } \\
400,000 \\
\text { Business: } \\
1,500,000\end{array}$ & $\begin{array}{c}\text { Real estate } \\
400,000 \\
\text { Business: } \\
1,500,000\end{array}$ \\
\hline Dominica 3/ & 100,000 & 200,000 & & $\ldots$ & $\cdots$ & 50,000 & 115,000 & $\begin{array}{c}\text { Real estate: } \\
200,000\end{array}$ & $\begin{array}{c}\text { Real estate } \\
200,000\end{array}$ \\
\hline Grenada & $\ldots$ & $\ldots$ & & 200,000 & 200,000 & 50,000 & 50,000 & $\begin{array}{c}\text { Real estate: } \\
350,000\end{array}$ & $\begin{array}{c}\text { Real estate } \\
350,000\end{array}$ \\
\hline St. Kitts and Nevis 4/ & $\ldots$ & $\ldots$ & & 250,000 & 300,000 & 50,000 & 125,000 & $\begin{array}{c}\text { Real estate: } \\
400,000\end{array}$ & $\begin{array}{c}\text { Real estate } \\
400,000\end{array}$ \\
\hline St. Lucia 5/ & ... & $\ldots$ & & 200,000 & 250,000 & $\ldots$ & $\ldots$ & $\begin{array}{c}\text { Real estate: } \\
\text { 300,000 } \\
\text { Gov. Bonds: } \\
\text { 500,000 } \\
\text { Business: } \\
\text { 3,500,000 }\end{array}$ & $\begin{array}{c}\text { Real estate } \\
300,000 \\
\text { Gov. Bonds } \\
550,000 \\
\text { Business: } \\
6,000,000\end{array}$ \\
\hline
\end{tabular}

Sources: Country Authorities; Citizenship by Investment Units Guidelines; Henley and Partners and Arton Capital.

1/ Depicts minimum Investment requirements for single vs. family applications (a couple with up to two dependents under the age of 18). Additional due diligence and processing fees apply.

2/ A limited time offer that remained valid from the launch of the program in 2013 through end-April 2016 allowed for a flat government processing fee of USD100,000 for a family of four, waiving the processing fees for the two dependents.

3 / Investment requirements for Dominica's CIP are expected to be revised upwards in $2016 \mathrm{H} 2$.

4/ Although an explicit government application fee is not required in the NDF option of St. Kitts and Nevis, about 25 percent of the contribution is retained by the government as budgetary fees.

5/ Business investment must fall under one of the following categories: Specialty Restaurants, Cruise ports and marinas, Agro-processing plants, 6 / For most programs, a minimum holding period of 5 years is required for redeemable investment options. Assets maybe eligible for resale to future applicants under the CIP. 


\section{Annex II. ECCU Risks to Financial Stability ${ }^{1}$}

\section{This annex evaluates risks to ECCU financial stability using the country financial stability map methodology, and credit cycle and financial soundness indicators heatmap. ${ }^{2}$}

Financial stability methodology maps quantitative indicators into six categories of risks and conditions relevant for financial stability: (i) macroeconomic risks, (ii) inward spillover risks, (iii) credit risks, (iv) market and liquidity risks, (iv) monetary and financial conditions, and (vi) risk appetite. Domestic and external sources of risks to financial stability and their evolution since a year ago are evaluated and compared against developments in global risks to financial stability from the Global Financial Stability Report.
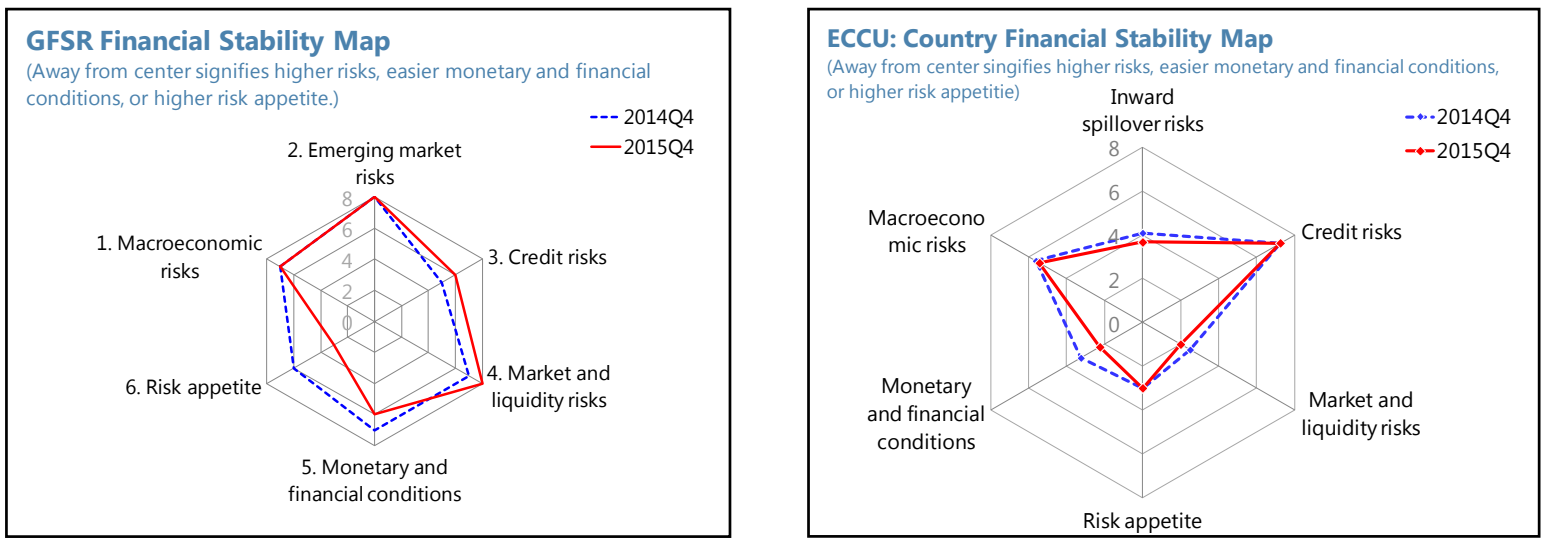

\section{Risks to ECCU financial stability remain largely unchanged and mainly originate from}

domestic sources. Credit risks associated with the fragile banking system continue to dominate domestic risks to the ECCU financial stability. While there is significant variation between the member states, on average, over the past year, credit risks have held up, given the weak balance sheets, declining credit, and elevated levels of delinquent loans, despite the marginal improvement in profitability. Macroeconomic risks remained largely unchanged as gains in lower output gap, marginally improved debt position, and lower current account deficit, were offset by further declining credit to private sector and slower economic growth. Inward spillover risks have largely remained unchanged as the positive effect of higher accumulation of banks' foreign assets offset slower exports growth. Monetary and financial conditions have tightened marginally on the back of higher real interest rates due to deflationary pressures, which tended to negate the monetary easing caused by the reduction in the minimum saving rate. Meanwhile, market risk appetite largely remained unchanged from a year ago. The evolution of risks to ECCU financial stability can be compared with that of global risks to financial stability of over the same period. The key difference is that ECCU risk appetite has largely remained the same, in contrast to lower global risk appetite.

\footnotetext{
1 Prepared by Alla Myrvoda.

2 ECCU financial stability maps are constructed using the methodology of Cervantes, Jeasakul, Maloney and Ong (2014) "Mapping Country to Global Financial Stability," IMF Working Paper, 2014.
} 
3. To assess the credit cycle and the soundness of the financial system in ECCU, we employ the use of credit cycle and financial soundness indicators (FSIs) heatmap tool. The tool incorporates indicators of the current soundness of the financial system and of its corporate and household counterparties, and produces a heat map of the credit cycle and key FSIs to inform whether policies need to adjust. It provides a snapshot of the three basic properties of the banking sector: credit cycle, balance sheet risks, and loss-absorbing capital buffers. A red indicator signifies the need for policy adjustment when the upper range of the threshold is breached; yellow coloring implies the state of alert when the indicator falls between the upper and the lower bound; while green signifies a 'no policy adjustment' scenario when the indicator is below the lower threshold. When applied to ECCU economies, however, the tool should be used with caution, given that it was developed to assess credit booms, causing many ECCU indicators to turn green and point to a low risk rating, owing to the protracted decline in credit.

4. ECCU credit cycle and balance sheet risks remain elevated and merit close watch. Credit to private sector declined further in 2015 , while excess liquidity continued to accumulate, driven by robust deposits. Inferior loan quality continues to persist, as marked by high levels of NPLs, while the balance sheet buffers remain vulnerable, given the low profitability of the banking system, despite the early signs of recovery. Credit declined by 4.2 percent (year-on-year) in February 2016.

\section{Banks' balance sheet data show sufficient} liquidity and contained foreign exchange risks. The ECCU deposit-to-loan ratio stabilized in 2015, but declined in the first months of 2016. The balance sheet buffers remain vulnerable as low profitability points to banks' limited ability to absorb negative

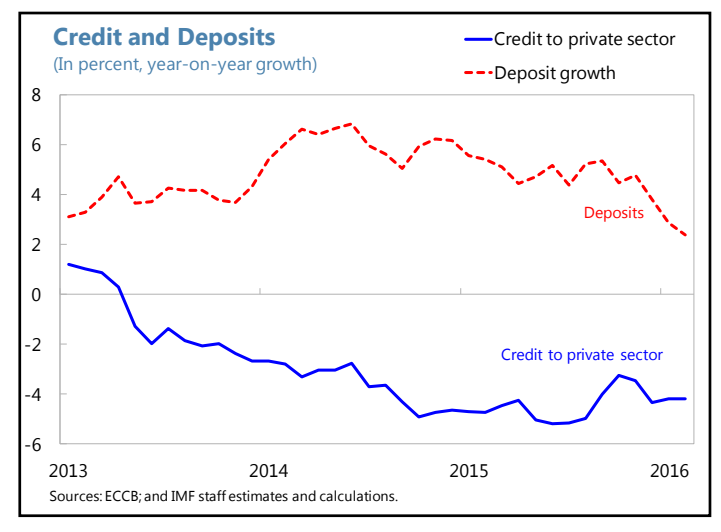
shocks. 
Table 1. ECCU Financial Soundness Indicators (FSIs) Heatmap

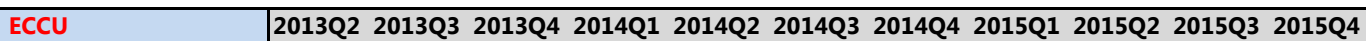

\begin{tabular}{|c|c|c|c|c|c|c|c|c|c|c|c|}
\hline Overall Financial Sector Rating & L & M & M & M & M & M & M & L & L & L & L \\
\hline Credit cycle & L & L & L & L & L & L & L & L & L & L & L \\
\hline Change in credit / GDP ratio ( $\mathrm{pp}$, annual) & (3.7) & (4.4) & $(5.5)$ & (8.6) & (7.3) & (7.8) & $(7.2)$ & $(5.2)$ & (5.8) & $(4.9)$ & (5.1) \\
\hline Growth of credit / GDP (\%, annual) & $(4.1)$ & $(5.0)$ & $(6.3)$ & (9.8) & $(8.5)$ & $(9.2)$ & (8.8) & (6.6) & (7.4) & (6.4) & (6.8) \\
\hline Credit-to-GDP gap (st. dev) & $(0.7)$ & $(0.7)$ & $(1.0)$ & (1.6) & $(0.6)$ & $(0.5)$ & $(0.1)$ & 0.5 & 0.9 & 1.3 & 1.2 \\
\hline Balance Sheet Soundness & L & M & M & M & M & M & M & L & L & L & L \\
\hline Balance Sheet Structural Risk & L & L & L & L & L & L & L & L & L & L & L \\
\hline Deposit-to-loan ratio & 124.2 & 129.2 & 130.4 & 137.4 & 138.2 & 140.6 & 144.8 & 151.6 & 154.4 & 154.4 & 154.4 \\
\hline FX liabilities \% (of total liabilities) & 21.1 & 20.4 & 19.7 & 19.5 & 20.1 & 20.3 & 20.1 & 20.5 & 20.1 & 20.5 & 19.7 \\
\hline FX loans \% (of total loans) & 17.5 & 18.1 & 18.0 & 17.7 & 17.7 & 17.8 & 17.7 & 17.6 & 16.8 & 16.8 & 16.6 \\
\hline Balance Sheet Buffers & L & M & M & M & $\mathrm{H}$ & M & M & L & L & L & L \\
\hline Leverage & L & L & L & L & M & M & M & M & M & M & L \\
\hline Leverage ratio (\%) & 8.3 & 8.0 & 7.9 & 7.7 & 6.9 & 6.3 & 6.2 & 5.8 & 6.1 & 6.1 & 8.1 \\
\hline Profitability & L & $\mathrm{H}$ & $\mathrm{H}$ & L & $\mathrm{H}$ & $\mathrm{H}$ & $\mathrm{H}$ & L & L & $\mathrm{L}$ & L \\
\hline ROA & 0.1 & $(0.1)$ & $(0.0)$ & 0.2 & $(0.0)$ & 0.1 & $(0.0)$ & 0.1 & 0.2 & 0.2 & 0.3 \\
\hline ROE & 0.4 & (2.9) & $(0.2)$ & 1.1 & 0.4 & $(0.5)$ & $(0.3)$ & 1.5 & 1.8 & 2.2 & 2.5 \\
\hline Asset quality & M & $\mathrm{H}$ & $\mathrm{H}$ & $\mathrm{H}$ & $\mathrm{H}$ & M & L & L & L & L & L \\
\hline NPL ratio & 15.7 & 18.5 & 18.3 & 19.3 & 18.6 & 18.5 & 17.9 & 18.6 & 18.5 & 18.1 & 17.0 \\
\hline NPL ratio change $(\%$, annual) & 7.9 & 29.6 & 20.5 & 19.9 & 18.5 & 0.1 & $(2.3)$ & (3.7) & $(0.5)$ & $(2.2)$ & $(4.8)$ \\
\hline \multicolumn{12}{|l|}{ Memo items: } \\
\hline Credit-to-GDP (\%) & 85.8 & 84.3 & 82.2 & 79.2 & 78.5 & 76.6 & 75.0 & 73.9 & 72.6 & 71.7 & 69.9 \\
\hline Credit-to-GDP gap (\%; HP filter) & 83.4 & 82.7 & 81.8 & 80.9 & 80.0 & 79.0 & 78.0 & 76.9 & 75.8 & 74.8 & 73.7 \\
\hline Credit growth (\%; annual) & $(2.0)$ & $(2.1)$ & $(2.7)$ & (3.3) & $(2.8)$ & (4.3) & (4.6) & $(4.5)$ & $(5.2)$ & $(4.0)$ & (4.3) \\
\hline CAR (in \%) & 13.7 & 12.4 & 12.1 & 12.0 & 10.5 & 10.5 & 10.2 & 9.7 & 10.4 & 10.6 & 14.0 \\
\hline Tier 1 CAR (in \%) & 12.9 & 11.5 & 11.3 & 11.2 & 9.7 & 10.1 & 9.9 & 9.5 & 9.8 & 10.5 & 13.4 \\
\hline
\end{tabular}

Sources: ECCB; and IMF staff estimates and calculations.

Note: This tool incorporates indicators of the current soundness of the financial system and of its corporate and household counterparties, and produces a heat map of the credit cycle and key FSIs to inform whether policies are needed. It provides a snapshot of the three basic properties of the banking sector: credit cycle, balance sheet risks, and loss-absorbing capital buffers. A red indicator signifies the need for policies when the upper range of the threshold is breached; yellow coloring implies the state of alert when the indicator falls between the upper and the lower bound; while green signifies a 'no policy' scenario when the indicator is below the lower threshold. When applied to ECCU, however, the tool should be used with caution, given that it was developed to assess credit booms, causing many ECCU indicators to turn green and point to a low risk rating. 


\section{Annex III. Spillovers from Trinidad and Tobago to ECCU 1}

This annex assesses the extent to which the slowdown of the real economic growth of Trinidad and Tobago, instigated by the oil price decline, may result in negative spillovers to feedback effects on the ECCU. Considerations of trade, tourism, and financial linkages, while relying on empirical evidence, point to a mixed effect on the ECCU economies. Thus, because of strong tourism and trade linkages, the spillovers to Grenada, Dominica, and St. Vincent and the Grenadines are estimated to be significant, while the effect on other countries within the ECCU is likely to be limited.

1. Falling energy prices put a strain on the Trinidadian economy in 2015. Falling energy prices are estimated to have resulted in a drop in oil and gas output by 4.9 percent $(y / y)$, and a decline in aggregate GDP by 2.1 percent. Lower energy prices also put a strain on the fiscal sector driving down the overall fiscal balance further into deficit. Lower oil and gas exports shifted the external current account from a surplus of 4.6 percent of GDP in 2014 to a deficit of 5.4 percent in 2015. Banking sector data through end-2015 showed no signs of deterioration in asset quality, with NPLs below $3 \frac{1}{2}$ percent of total loans.

Meanwhile banks' balance sheets remained liquid, with relatively high capital buffers and profitability,

Trinidad and Tobago: Real GDP Growth (In percent, year-on-year; actual 2012Q1-15Q3)

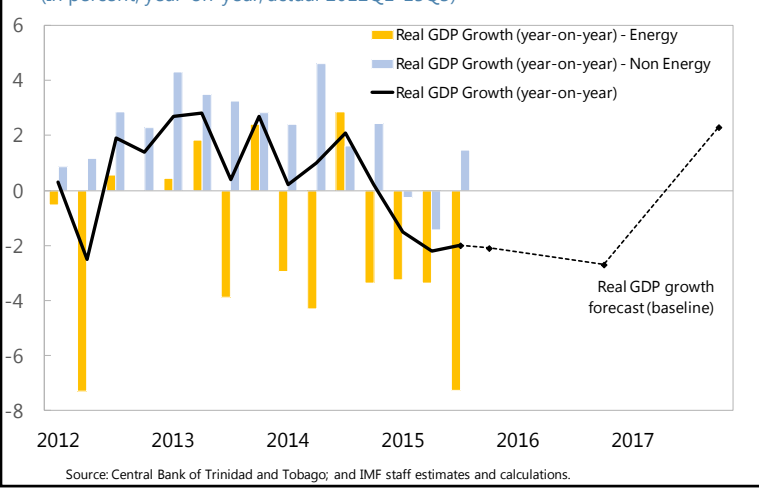
despite some declines in returns.

\section{The main linkages between the ECCU and Trinidad and Tobago are largely observed} through the trade in goods, tourism, and financial sector interconnectedness. Some anecdotal evidence suggests that Trinidadian institutional investors, including banks, hold RGSM securities. While the level of involvement of the Trinidadian investors is difficult to determine market participants report that Trinidadian investors may be holding about 5 to 10 percent of ECCU government securities and there has been no significant withdrawal of Trinidadian institutional investors from the regional government securities market (RGSM).

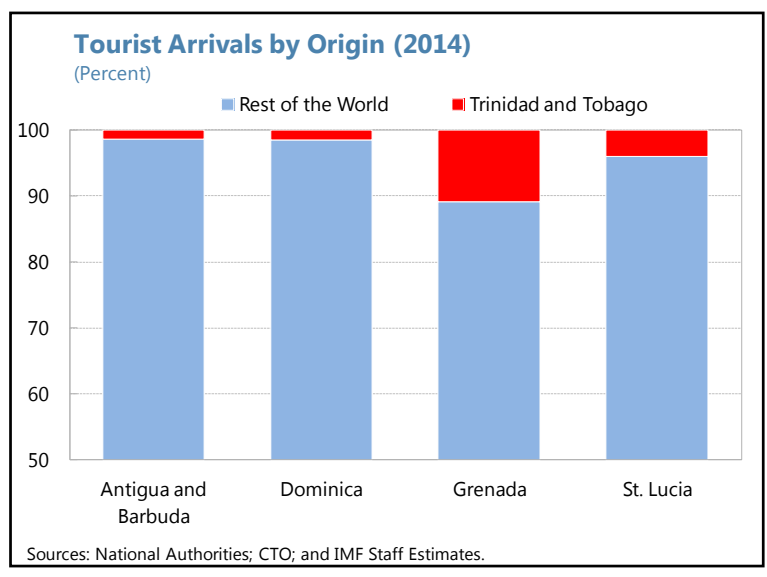
The relatively strong health of the Trinidadian banking sector indicates that the negative spillovers to the ECCU are likely to be contained - at least over the short term-and Trinidadian banks are unlikely to have to sell off their holdings of RGSM securities given sufficient capital and liquidity

\footnotetext{
${ }^{1}$ Prepared by Alla Myrvoda and Mauricio Vargas.
} 
buffers. However, a protracted period of slower growth in Trinidad and Tobago may dampen demand for RGSM securities. Some authorities have reported that foreign exchange shortages in Trinidad and Tobago have created delays in remittance transfers and payments for exports.

\section{While the spillovers through the banking sector may be cushioned by the relative} robustness of the Trinidadian banking sector, spillovers through the tourism and trade sectors are likely to be more pronounced. Since a few countries receive a significant portion of tourist arrivals from Trinidad and Tobago, the slowdown in Trinidadian economic activity is likely to restrain Trinidadian travel to the ECCU. For example, about 11 percent of tourists to Grenada are from Trinidad and Tobago. Spillovers through the trade channel, on the other hand, are likely to vary greatly by country, as some countries, such as Dominica and St. Vincent and the Grenadines export a significant portion of their exports to Trinidad and Tobago.

\begin{tabular}{|c|c|c|c|c|}
\hline & \multicolumn{3}{|c|}{$\begin{array}{l}\text { Share of exports to } \\
\text { Trinidad and Tobago } \\
\text { (percent of total) }\end{array}$} & \multirow{2}{*}{$\begin{array}{l}\text { Share of tourist from } \\
\text { Trinidad and Tobago } \\
\text { (percent of total) }\end{array}$} \\
\hline & 2000 & 2005 & 2014 & \\
\hline Antigua and Barbuda & 0.0 & 0.2 & 2.8 & 1.4 \\
\hline Dominica & 3.0 & 10.5 & 35.9 & 1.6 \\
\hline Grenada & 0.2 & 0.9 & 2.1 & 11.0 \\
\hline St. Kitts and Nevis & 0.5 & 0.1 & 0.3 & $\ldots$ \\
\hline St. Lucia & 1.6 & 5.9 & 7.0 & 4.1 \\
\hline St. Vincent and the Grenadiens & 8.2 & 14.0 & 35.2 & $\ldots$ \\
\hline ECCU & 2.2 & 4.1 & 11.6 & $\ldots$ \\
\hline
\end{tabular}

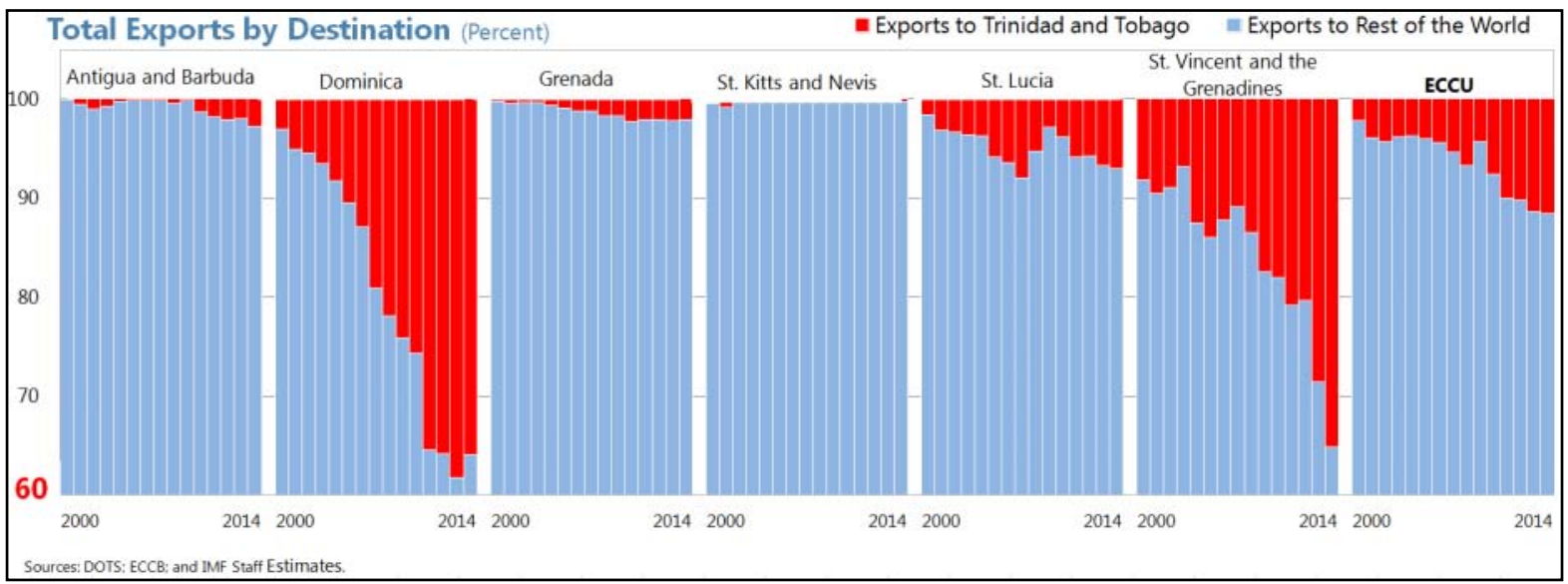

4. Econometric analysis corroborates the finding that some ECCU economies with tighter trade and tourism linkages to Trinidad and Tobago may experience larger spillovers. Staff' used a variant of the global vector autoregressive model (GVAR) to assess spillovers from Trinidad and Tobago to the ECCU. ${ }^{2}$ The model assumes a number of transmission channels, including the

\footnotetext{
2 The GVAR was originally proposed by Pesaran, Schuermann, and Weiner (2004). Model estimators are based on annual data from 1980 to 2014. The sample includes: Antigua and Barbuda, Dominica, Grenada, St. Kitts and Nevis, St. Lucia, St. Vincent and the Grenadines, Canada, Trinidad and Tobago, United Kingdom, USA, Finland, Barbados, China, France, Germany, Italy, Japan, Spain, Singapore, Poland, Cameroon, Dominican Republic, and Mexico. The GVAR model estimates an individual VARX model for each of the countries (23 VARX models). Each VARX model, in its default specification, includes a set of endogenous variables (GDP,
}

(continued) 
effect of a shock to Trinidad and Tobago on real GDP growth of the ECCU though changes in trading partners" GDP (similarly to a gravity model); trading partners' REER; and trade and tourism, among others. The impulse response functions obtained from the GVAR model suggest that a positive shock of 1 percent to the GDP of Trinidad and Tobago would increase ECCU GDP by about 0.12 percentages points. ${ }^{3}$ The magnitude of the effect, however, varies by country. Thus, the results indicate that Grenada may experience a greater shock to its GDP, meanwhile the effect on

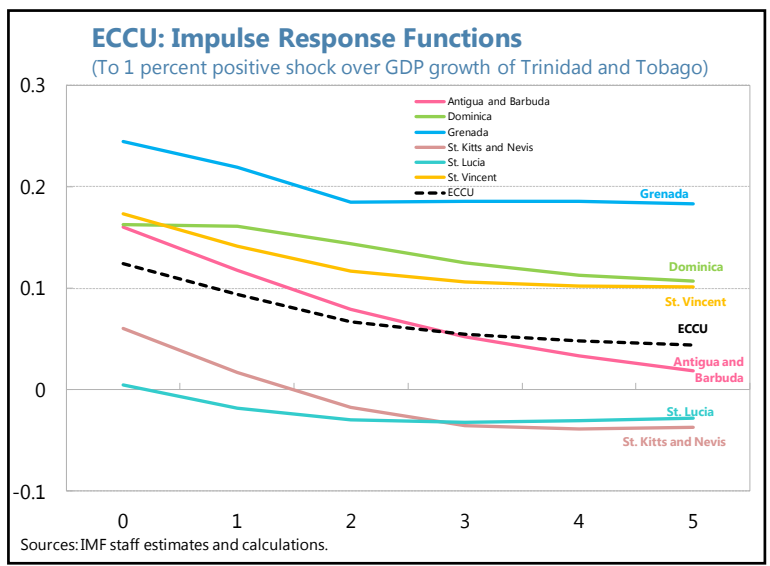
St. Kitts and Nevis and St. Lucia is negligible. While the relatively strong response in Grenada is driven largely by tourist arrivals, the effect on Dominica and St. Vincent is likely to stem from the high share of exports to Trinidad and Tobago.

Inflation, Short term Interest Rate, and Real Exchange Rate); and exogenous variables (the "relative-to-trading-partners" counterpart of the 4 endogenous variables)t; and two global variables: an oil price index and an index of price of raw materials.

\footnotetext{
${ }^{3}$ After combining the 23 VARX individual models (by using a matrix of trading-partner weights), the GVAR allows simulating the effects of a shock in any of the local variables or the two global variables over the rest of the system. The impact of a one-time positive shock in the GDP of Trinidad and Tobago on the GDP of the ECCU countries is obtained then using impulse response functions. While the GVAR model does not provide an analytical solution to calculate confidence intervals, the sign of the effect is robust to the model specification.
} 


\section{Annex IV. Safeguarding Financial Stability in ECCU1}

\section{Following the Global Financial Crisis, ECCU banks experienced substantial financial} distress. The financial position of the indigenous (domestic) and foreign-owned banks showed marked deterioration in asset quality, capital, and earnings, with the position of the indigenous banks generally weaker than the foreign-owned banks. At end-2013, reported aggregated NPLs reached 18 percent of total loans, well above the prudential benchmark of 5 percent, with those of indigenous banks at 25 percent, twice that of foreign owned banks. As of late 2015, three insolvent banks were under ECCB conservatorship: Antigua Barbuda Investment Bank (ABIB) in Antigua; and Caribbean Commercial Bank, (CCB), and National Bank of Anguilla (NBA) in Anguilla.

\section{A three-pronged strategy was put in place, aimed at addressing the main issues threatening regional financial stability:}

- $\quad$ A hybrid purchase and assumption (P\&A) structure was implemented to resolve the three insolvent banks, while allowing fiscally constrained governments to fulfill their commitment on protecting all domestic deposits. The P\&A also contemplated the creation of a regional asset management company (ECAMC).

- $\quad$ An asset quality review (AQR) was conducted across all indigenous banks, along with a dynamic modeling of banks' business model. The AQR revealed important underprovisioning in several indigenous banks, which is to generate supervisory actions and capital calls in the near-term, supported by the dynamic modeling results.

- Substantial technical assistance has been (and continues to be) provided by the International Financial Institutions (IFIs) to strengthen the ECCB's supervision, including through the presence of a long-term expert at the ECCB, hired by the IMF. ${ }^{2}$

\section{The resolution of the three banks under ECCB conservatorship was repeatedly delayed,} resulting in a serious threat to financial stability in the region. $A B I B$ was placed under conservatorship in 2011, and CCB and NBA in 2013. All three banks were deeply insolvent, albeit not facing liquidity shortages or runs. There was a legitimate concern that a confidence shock could trigger not only a deposit run on the three banks, but also a widespread uneasiness among depositors in all islands. Resolving the three banks as soon as possible, thus, became imperative.

\section{Potential solutions faced important constraints, as authorities committed to protect all} deposits, but governments had no fiscal space. Under a standard P\&A, a successor bank purchases some (or all) the good assets, while assuming matching liabilities. Remaining liabilities and assets go into receivership and are repaid over time with recoveries, in accordance to a claimholders priority list. This type of structure would not allow authorities to fulfill their

\footnotetext{
1 Prepared by Michael Moore and Marcos Souto.

2 The three IFIs involved in providing technical assistance to the ECCB were the IMF, the World Bank, and the Caribbean Development Bank. Technical assistance has been mostly funded by the Canadian Government.
} 
commitment of protecting all deposits, which could generate a confidence shock across all islands, because there were not enough good assets to match all deposits and the governments of Antigua and Barbuda (GoAB) and of Anguilla (GoA) did not have fiscal space to repay all deposits. Hence, a comprehensive structure was designed, with the creation of a special purpose vehicle (a deposit protection trust, DPT) that would house deposits not transferred to a successor good bank (or to receivership), above a certain threshold, funded by a long-term government bond, fitting within the GoA's and GoAB's fiscal envelopes. Recoveries on distressed assets are to be deposited in a sinking fund held at the ECCB and used to minimize the fiscal costs associated with servicing the bonds (Figure 1)

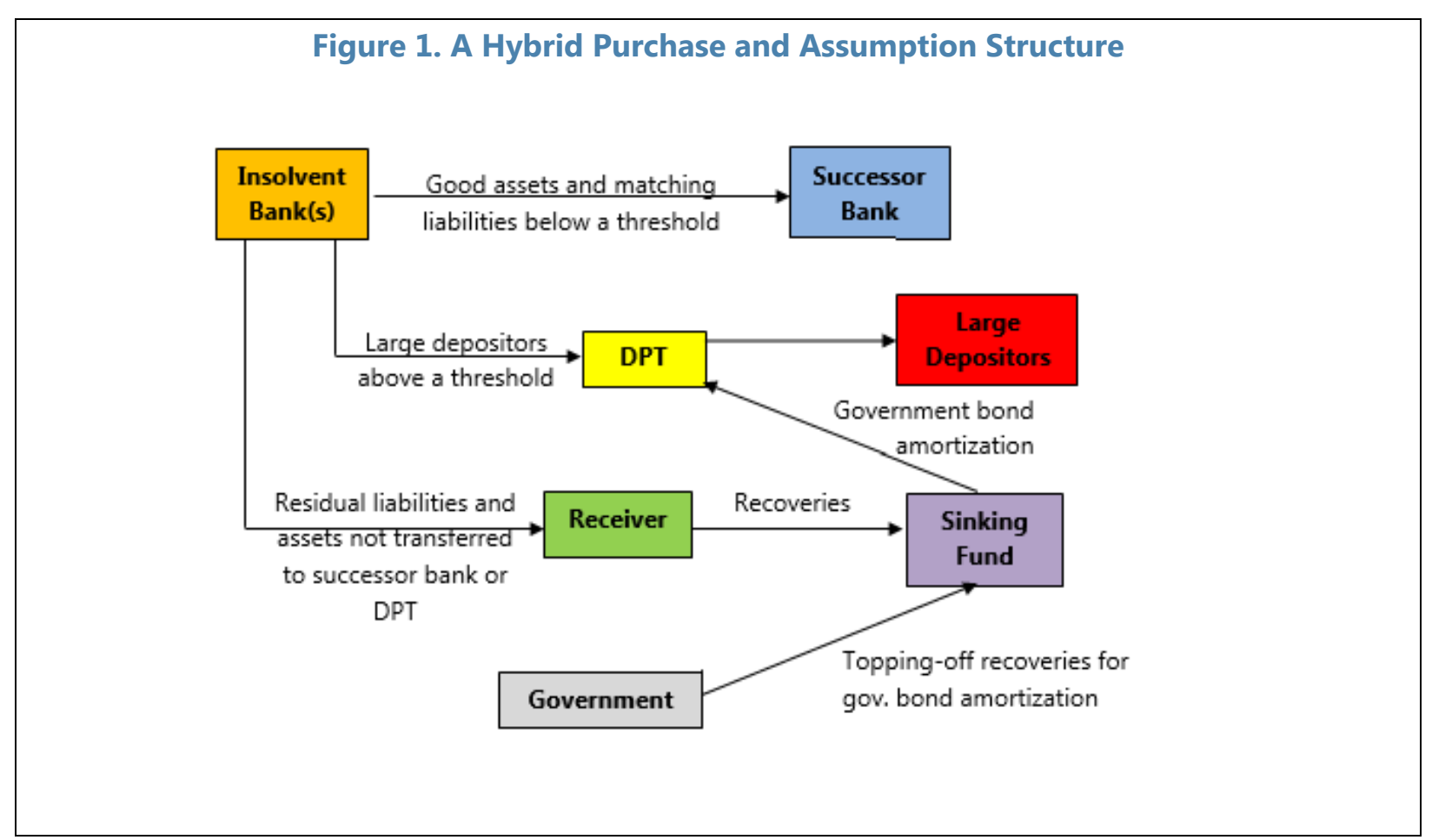

\section{Resolution of ABIB}

\section{The successful resolution strategy for ABIB involved a P\&A with the Eastern Caribbean}

Amalgamated Bank (ECAB). The closure of ABIB on November 27, 2015 went very well with negligible deposit withdrawals. Good assets were purchased by ECAB along with matching liabilities, allowing deposits up to $\mathrm{EC} \$ 500,000$, as well as government deposits, to be transferred to $E C A B$, with the remaining deposits transferred to the DPT. GoAB issued a 10-year amortizing with a coupon of 2 percent to the DPT. The GoAB also purchased back the equivalent to US $\$ 30$ million in government debt from ECAB, using the first tranche of a loan from the Caribbean Development Bank, and committed to purchase another US $\$ 20$ million by September 2016 , with the disbursement of the 
second tranche. ${ }^{3}$ This feature of $A B I B$ 's resolution helped further improve ECAB's liquidity position and reduced $E C A B^{\prime} s$ exposure to the GoAB, which had been a concern for the bank. All remaining assets and liabilities not transferred to ECAB or to the DPT were transferred to a local receiver. Once the ECAMC is up and running, it is supposed to take over the receiverships in Antigua and Anguilla.

\section{Resolution of CCB and NBA, Anguilla}

\section{The resolution of CCB and NBA involved the creation of a bridge bank to warehouse} good assets, some nonperforming loans (NPLs) and matching liabilities. The resolution structure for CCB and NBA was very similar to ABIB's and it also went very well with no deposit run (CCB and NBA were closed on April 22, 2016). Good assets and some NPLs, along with matching deposits, were transferred to create a bridge (good) bank-the GoA-owned National Commercial Bank of Anguilla (NCBA). As CCB and NBA had relatively more good assets than ABIB, a higher threshold for deposit transfer (EC\$2.8 million) was possible. Deposits above the threshold, amounting to EC\$52 million, were transferred to two DPTs, one for each of the closed banks, to be matched by a 10-year GoA amortizing bond paying a 2 percent annual coupon. ${ }^{4}$ The remaining assets and liabilities were transferred to two receiverships, one for each of the closed banks, including placements from off-shore subsidiaries and deposits from the Social Security Board (SSB). ${ }^{5}$ Repayment of the EC $\$ 208$ million in SSB deposits will be funded by a 20 -year amortizing bond paying a 3 percent coupon, to be issued by the GoA.

\section{Next Steps}

7. Consistent with the strategy agreed with IFIs, the next steps are: finishing the resolution of ABIB, NBA and CCB toward the transition of their receiverships to the ECAMC and continuing efforts to address the high NPLs that trouble other ECCU banks:

- Properly setting up and preparing $A B I B, N B A$ and $C C B$ receivers' transition to the ECAMC is key to the success of the strategy. The ABIB, NBA and CCB closings went well, with the support of IFIs' technical assistance and external consultants hired by the $E C C B$, who assisted the receiver in Anguilla in setting up the proper controls at the receiverships (e.g., to prevent asset stripping). The receiver in Antigua has already been

\footnotetext{
3 The CDB provided a loan to the GoAB of US $\$ 50$ million (EC $\$ 135$ million), to be repaid in quarterly installments over 12 years (after a five-year grace period), with a 3.4 percent floating interest rate. The first tranche of the loan (US\$ 30 million or EC\$81 million) was disbursed in December 2015, after the GoAB signed and submitted a letter to Fund staff, setting out its commitment to implement fiscal measures to enhance the sustainability of the fiscal position and support the repayment of the government bond issued to the DPT. The second disbursement is scheduled for December 2016, predicated on a positive assessment by the Fund staff.

${ }^{4}$ From a legal standpoint there needs to be two receiverships, as claimholders to CCB are entitled to CCB assets' recoveries only. The DPT structure must mirror those for the receiverships, as DPTs have a subrogation claim to the recoveries of assets in the receiverships.

${ }^{5}$ A contentious issue has been the EC $\$ 147$ million in deposits at the CCB and NBA off-shore subsidiaries that were placed at the respective on-shore parents. The current provisional administrator appointed to manage the off-shore subsidiaries has threatened to take ECCB to court, as these placements are currently allocated in the receivership, until a legal opinion or judicial decision rules it to be transferred somewhere else.
} 
set up with external technical assistance support. Key to the durable resolution will be that $A B I B, N B A$ and $C C B$ receiverships be transferred properly to the ECAMC when it becomes operational.

- $\quad$ ECAMC must be established as soon as possible. The needed legislation to establish the ECAMC is now a law in all ECCU countries. The ECCB is coordinating the final elements to establish the ECAMC, to be headquartered in Antigua, which are: confirmation of new directors; contribution of capital from ECCU member jurisdictions; and hiring of staff.

- The system NPLs must be addressed in a comprehensive and effective manner. The ECAMC will need to be effective recovering distressed assets so it can be well-placed to convince other ECCU banks to sell some of their NPLs. It will be critical that loan purchases are on commercial terms. Key to the ECAMC's effectiveness will be the successful application of its special legislative powers to restructure troubled assets. 


\section{Annex V. Non-Performing Loans in the ECCU: Determinants and Macroeconomic Impact ${ }^{1}$}

This annex assesses the determinants of NPLs in the ECCU and the extent to which the deterioration in asset quality may result in negative feedback effects from the banking system to economic activity. The results suggest that the deterioration in asset quality can be attributed to both macroeconomic factors, including the prolonged recession in the region following the global financial crisis and slow pace of economic recovery, and bank-specific factors. Banks with stronger profitability and lower exposure to the volatile construction and tourism sectors and household loans tend to have lower NPLs. There is also some evidence that foreign owned banks systematically have lower NPLs than indigenous banks Finally, the results emphasize the importance of macro-financial feedback loops in the ECCU. Improved asset quality will be key to reverse these negative feedback loops and support sustained economic growth and, similarly, stronger economic growth will be imperative to strengthen asset quality and financial stability.

\section{A. Introduction}

1. NPLs are elevated across the ECCU, with NPL ratios well above the prudential guideline of 5 percent in all jurisdictions. The high level of NPLs appears to be, in part, a legacy of the global financial crisis, which burst the domestic credit cycle as it spilled over to the region. Prior to the crisis, credit had expanded rapidly, mainly spurred by economic activity in the tourism industry and related construction. The upward trend in NPL ratios continued beyond the end of the global crisis, reflecting the slow pace of economic recovery experienced by much of the region. In addition, country-specific factors have contributed to the spike in the NPL ratio in some countries. For example, the debt-for-land swap completed between the government and indigenous banks in St. Kitts and Nevis contributed to a sharp rise in the NPL ratio over 2011-2015. On a sectoral basis, the increase in the NPL ratio since the global financial crisis has been driven to a large extent by tourism, construction, and personal loans (which accounted for 18,18 and 43 percent of total loans at the end of 2015, respectively). Low profitability has restricted banks ability to increase provisioning, which

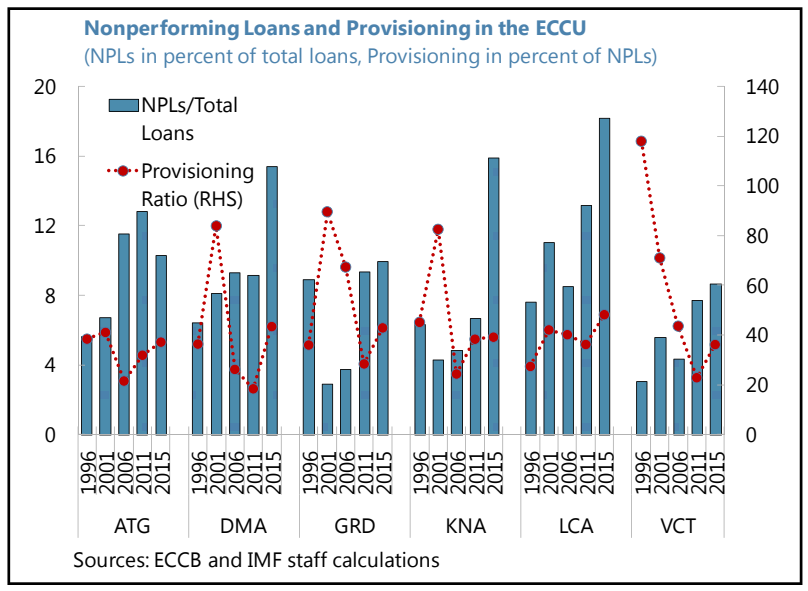
remains inadequate throughout the region.

\footnotetext{
${ }^{1}$ Prepared by Kimberly Beaton, Alla Myrvoda, and Hanlei Yun (all IMF) and Shernnel Thompson (Eastern Caribbean Central Bank).
} 


\section{Nonperforming Loans by Economic Sector (2015)}

The construction, tourism, and agriculture industries have the highest incidence of NPLs...

Nonperforming Loans in the ECCU by Economic Sector 1/ (in percent of total loans; by sector)

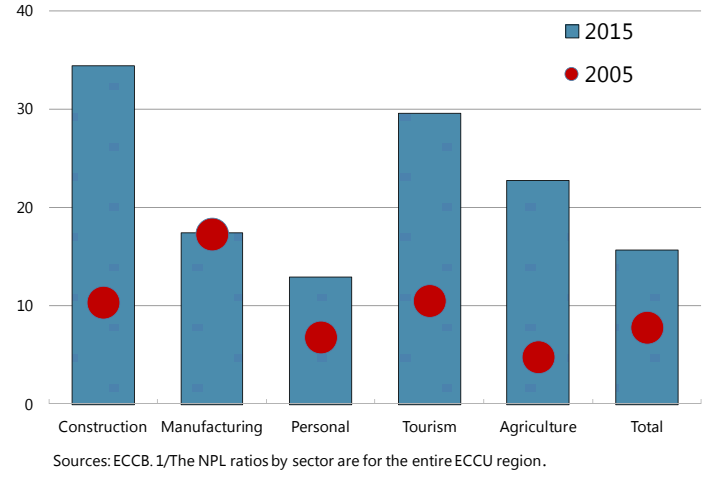

...While personal loans and credit to construction and tourism industries constitute the bulk of NPLs in ECCU.

ECCU: Distribution of NPLs by Sector (In percent of total)

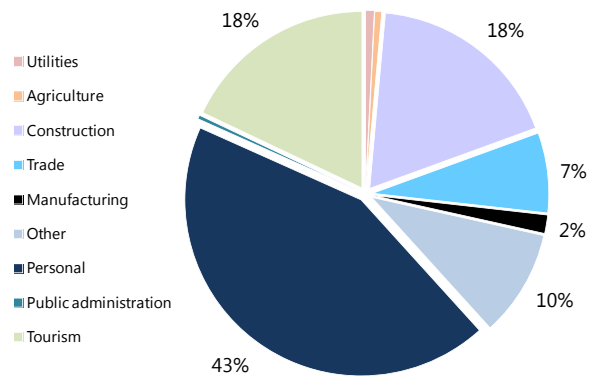

2. There is wide dispersion in the level of NPLs across individual banks, suggesting that bank-specific factors also contributed to the deterioration in asset quality in the ECCU. In 2015, the median NPL ratio across individual banks was 12.8 percent however, the lowest ratio was 4.5 percent and the highest was 24.8 percent. There is also considerable dispersion in the level of NPLs by ownership type. For most ECCU countries, indigenous banks tend to have higher NPL ratios. The exceptions are Grenada and St. Vincent and the Grenadines where the NPL ratio is higher for foreign-owned banks.

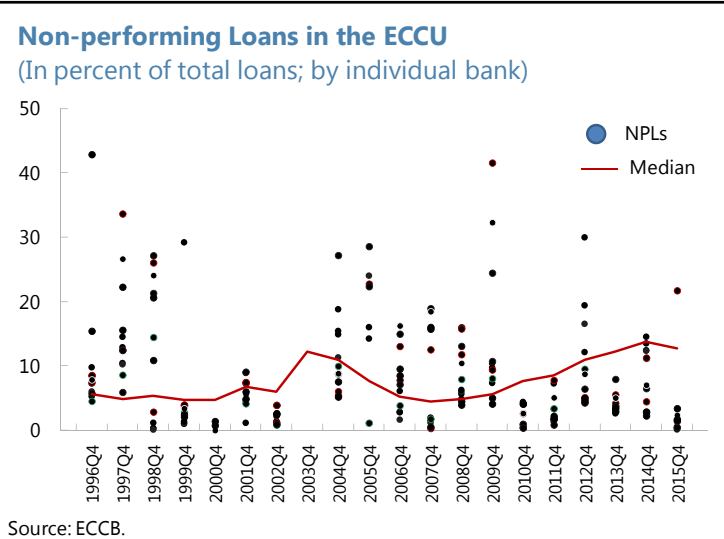




\begin{tabular}{|c|c|c|c|c|c|c|c|c|c|c|c|c|}
\hline \multicolumn{13}{|c|}{ NPLs Across Foreign and Indigenous Banks in the ECCU 1/ } \\
\hline & & 2005 & 2006 & 2007 & 2008 & 2009 & 2010 & 2011 & 2012 & 2013 & 2014 & 2015 \\
\hline \multirow{2}{*}{ Grenada } & Foreign & 3.5 & 2.4 & 3.5 & 3.1 & 5.8 & 6.4 & 8.9 & 12.0 & 13.7 & 15.4 & 10.7 \\
\hline & Indigenous & 6.8 & 4.7 & 3.4 & 5.0 & 6.6 & 12.6 & 11.3 & 10.8 & 14.2 & 11.9 & 7.5 \\
\hline \multirow{2}{*}{$\begin{array}{l}\text { Antigua and } \\
\text { Barbuda }\end{array}$} & Foreign & 4.2 & 4.8 & 3.6 & 3.9 & 4.9 & 7.9 & 8.3 & 12.6 & 9.0 & 9.2 & 8.6 \\
\hline & Indigenous & 20.5 & 18.2 & 15.6 & 25.0 & 10.8 & 14.1 & 19.1 & 19.7 & 21.3 & 21.0 & 12.4 \\
\hline \multirow{2}{*}{ Dominica } & Foreign & 15.8 & 11.6 & 10.1 & 9.4 & 5.3 & 8.6 & 9.1 & 10.2 & 12.4 & 13.3 & 13.6 \\
\hline & Indigenous & 32.3 & 5.9 & 5.0 & 4.1 & 5.6 & 8.9 & 9.2 & 14.8 & 15.2 & 14.9 & 16.8 \\
\hline \multirow{2}{*}{$\begin{array}{l}\text { St. Kitts and } \\
\text { Nevis }\end{array}$} & Foreign & 3.3 & 2.2 & 2.2 & 3.4 & 4.5 & 5.8 & 7.7 & 12.7 & 9.9 & 10.3 & 9.5 \\
\hline & Indigenous & 7.9 & 6.6 & 5.4 & 5.5 & 4.1 & 5.3 & 5.8 & 8.9 & 12.1 & 16.8 & 22.2 \\
\hline \multirow{2}{*}{ St. Lucia } & Foreign & 9.9 & 7.1 & 4.8 & 5.8 & 6.8 & 13.3 & 14.6 & 16.7 & 14.5 & 15.4 & 16.2 \\
\hline & Indigenous & 16.5 & 10.5 & 7.3 & 8.3 & 9.9 & 10.7 & 10.9 & 13.3 & 29.1 & 20.6 & 20.9 \\
\hline \multirow{2}{*}{$\begin{array}{l}\text { St. Vincent and } \\
\text { the Grenadines }\end{array}$} & Foreign & 4.7 & 4.8 & 4.1 & 5.4 & 8.3 & 8.8 & 8.1 & 8.7 & 10.0 & 13.3 & 11.1 \\
\hline & Indigenous & 5.9 & 3.9 & 2.8 & 2.2 & 8.5 & 8.3 & 7.1 & 5.8 & 6.2 & 6.2 & 6.0 \\
\hline
\end{tabular}

\section{More profitable banks tend to have lower}

NPL ratios. In the ECCU, stronger bank profitability (evidenced by banks' return on assets), which may be reflective of the quality of bank management, is correlated with lower NPL ratios. ${ }^{2}$ Of course, the causality also runs in the other direction as higher NPLs directly erode profitability through higher provisioning. Foreign-owned banks have generally exhibited stronger profitability and lower NPL ratios relative to indigenous banks.

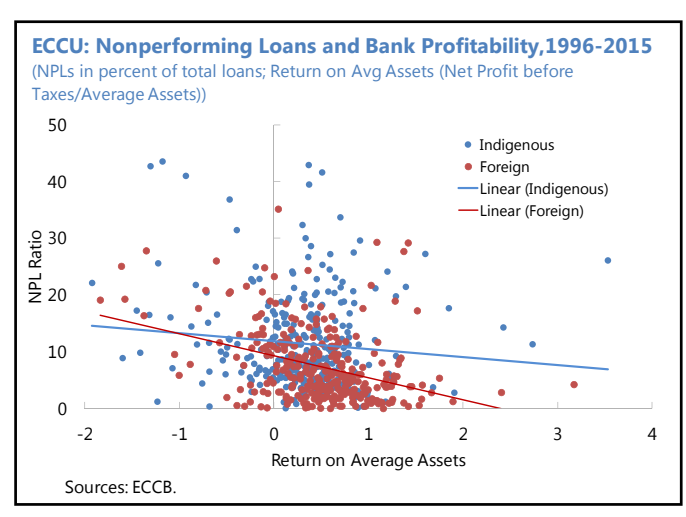

\section{Elevated NPLs may result in adverse macrofinancial feedback loops. In particular,} elevated NPLs may affect the real economy through the credit supply channel, as accumulating NPLs force banks to tighten their underwriting standards and limit the supply of credit to the private sector. Following the global financial crisis, credit terms and conditions tightened as banks restricted access to credit and focused on strengthening their balance sheets. Combined with weak economic fundamentals and demand for credit, the reduction in credit supply resulted in a contraction in credit to the private sector (primarily in the private business segment) that began in early 2013. Similarly, the erosion of bank asset quality and the associated contraction in credit have likely reinforced the region's subdued growth and contributed to adverse macro-financial feedback loops in the region. Indeed, higher NPLs tend to be correlated with both lower credit growth and weaker economic growth.

\footnotetext{
${ }^{2}$ The same dynamic is apparent if the net interest margin is considered as an alternative measure of bank profitability.
} 


\section{Nonperforming Loans and Economic Activity}

High NPLs continue to impede private sector access to credit, as credit growth has remained negative since early 2013.

ECCU: Nonperforming Loans and Credit Growth,1996-2015 (NPLs in percent of total loans; Credit Growth in percent, year-over-year) 50

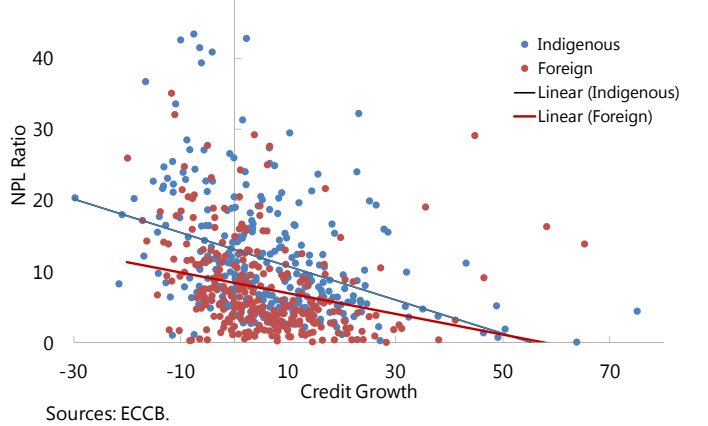

Economic recovery in the ECCU after the global financial crisis is yet to manifest itself in the banking sector, as credit continues to decline.

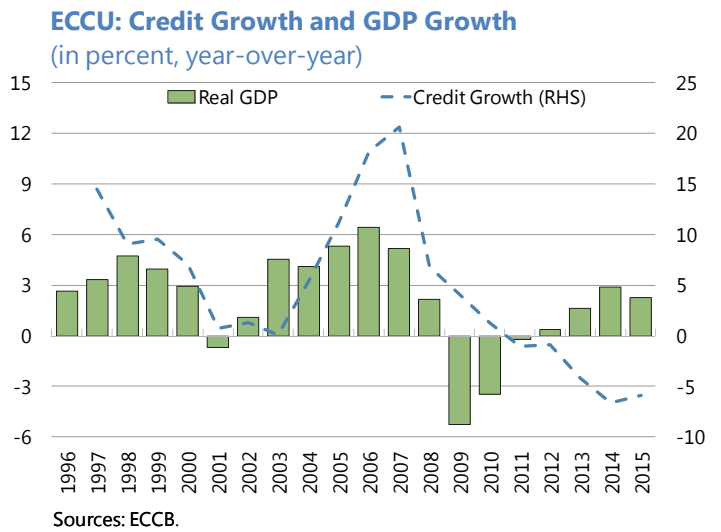

\section{B. The Determinants of Nonperforming Loans in the ECCU}

\section{Bank-level panel data are used to estimate the determinants of NPLs in the ECCU. The} quarterly dataset spans 1996Q1-2015Q4 and, uniquely, has universal coverage of all banks operating in the ECCU independent countries over this time frame, including all indigenous and foreign banks (branches and subsidiaries). ${ }^{3}$ The basic dynamic panel regression specification is:

$$
\begin{aligned}
& N P L s_{i, j . t}=\alpha+\beta_{1} N P L s_{i, j . t-1}+\beta_{2} \text { Global }_{t}+\beta_{3} \text { Country }_{i, t}+\beta_{4} \text { Bank }_{i, j, t}+\beta_{5} \text { Foreignbank }_{j}+ \\
& \beta_{6} \text { Country }_{i}+\varepsilon_{i, j, t}
\end{aligned}
$$

where $N P L s_{i, j . t}$ denotes the logit transformation of the NPL ratio for bank $i$ in country $j$ at time $t .{ }^{4}$ The dependent variable is explained by its lag $\left(N P L s_{i, j . t-1}\right)$, global $\left(G_{l o b a l}\right)$, country-specific (Country $\left._{i, t}\right)$, and bank-level $\left(\right.$ Bank $\left._{i, j, t}\right)$ variables. Institutional factors, such as the quality of bank supervision and financial regulation, may also be important determinants of NPLs but were not considered as explanatory variables, given that bank regulation and supervision is harmonized across the currency union with the ECCB as the regulator of the regional banking system. The

\footnotetext{
${ }^{3}$ The sample includes 2359 observation: 34 banks, six countries (Antigua and Barbuda, Dominica, Grenada, St. Kitts and Nevis, St. Lucia, and St. Vincent and the Grenadines); 80 periods (1996Q1-2015Q4). The data are aggregated by individual bank. Of the 34 banks in the sample, 12 are indigenous banks, 6 are subsidiaries of foreign banks and 15 are branches of foreign banks. The foreign banks are predominately branches and subsidiaries of Canadian banks, with a bank from Trinidad and Tobago also operating a subsidiary in Grenada. As of end-2015, foreign banks held 47 percent of total banking system assets (or 109 percent of GDP) and 40 percent of total deposits in the banking system (or 79 percent of GDP).

${ }^{4}$ This transformation ensures that the dependent variable spans the interval $[-\infty,+\infty]$ and is distributed symmetrically. It also ensures that all predicted values are non-negative and between 0 and 1 .
} 
specification also controls for individual country effects and the type of bank ownership by including a foreign bank (Foreignbank $D_{j}$ ) and country $\left(\right.$ Country $\left._{i}\right)$ dummy variable.

\section{The results of the baseline model (Table 1) confirm that macroeconomic developments} are important determinants of banks' asset quality. The baseline specification restricted to the macroeconomic determinants of NPLs suggests that both global and country-specific macroeconomic developments affect NPLs in the region. Asset quality in the banking system is affected by spillovers from global macroeconomic developments, with the results suggesting that stronger growth in advanced economies lowers NPLs in the ECCU, consistent with the high degree of openness of the small economies of the ECCU member countries. ${ }^{5,6}$ Somewhat conversely, tourism growth, included in the regression as a proxy for domestic economic activity due to the unavailability of data on quarterly GDP and unemployment for the region, is found to increase NPLs. This may be associated with the inherent riskiness of lending to the tourism sector; however, this result holds even when controlling for the concentration of banks' lending to the tourism sector. The results are robust to random effects estimation and fixed effects estimation, which controls for unobserved heterogeneity across individual banks. ${ }^{7}$

\section{Bank-specific variables are also found to be important determinants of NPLs. ${ }^{8}$ Higher}

bank profitability, captured by the return on assets, is found to lower NPLs. The analysis also controls for the composition of outstanding loans and finds that bank asset quality tends to decrease with a higher concentration of banks' lending portfolios in loans to households and the construction and tourism sectors. ${ }^{9}$ Contrary to expectations, lagged credit growth is found to result in lower NPLs, suggesting that the impact of credit growth on the denominator outweighs the impact of increased lending and its inherent riskiness on the numerator of the dependent variable. In contrast to previous studies, other indicators of bank efficiency (e.g. expense to income ratio), excessive lending (e.g. loans-to-assets and loans-to-deposit ratios) and interest rates were not found to have a significant impact on bank asset quality. NPLs were also found to have relatively high auto-correlation, suggesting that a shock to NPLs could have a prolonged effect on the ECCU banking system. These results are also robust to random and fixed effects estimation.

\footnotetext{
${ }^{5}$ While some studies have found that the exchange rate can have an impact on asset quality, due to the impact of exchange rate depreciation on borrowers' capacity to service their foreign currency loans, this is not investigated for the ECCU given the fixed exchange rate to the US dollar.

${ }^{6}$ Given the prevalence of natural disasters in the region, a dummy variable was also included to capture the potential impact of natural disasters on NPLs; however, it was not found to be significant.

7 The country and foreign bank dummies are excluded from the fixed effects estimation due to collinearity.

${ }^{8}$ Capital and equity variables were not considered in the baseline specification given the inclusion of foreign bank branches, which do not maintain local capital, in the dataset. Capital and equity measures were included in a regression restricted to indigenous banks and foreign subsidiaries and were not found to be significant determinants of bank asset quality in the ECCU.

${ }^{9}$ As measured by the logit transformation of the loans to households to total loans ratio and the sum of construction and tourism loans to total loans ratio. Tourism and construction are considered jointly given the concentration of construction activity in the tourism sector in the region. The concentration of bank lending to the government and private businesses were also investigated as potential determinants and not found to be significant.
} 
8. The global financial crisis contributed to the deterioration in bank asset quality in the ECCU. To evaluate the effect of the global financial crisis, a dummy variable for the period 20082015 was included in the baseline regression specification including and excluding bank-specific variables. In both instances, the dummy variable is positive and significant, suggestive of a structural increase in the level of NPLs in the region since the global financial crisis.

\section{The baseline results suggest that the type of bank ownership has an impact on NPLs.}

The foreign bank dummy variable is significant with a negative coefficient in both the baseline specification with and without bank-specific factors and regardless of whether the time dummy is included or excluded from the model specification. This result suggests that there are some institutional features in the business model of indigenous banks that are not captured by the bankspecific variables that contribute to higher NPLs than in foreign banks. These could include differences in due diligence and/or risk management practices as well as differences in banks' strategies to recover on NPLs. To explore in more detail the impact of ownership structure on NPLs, the foreign bank dummy variable was interacted with all other variables in the benchmark model. The general specification of the model with interactions is:

$N P L s_{i, j, t}=\alpha+\beta_{1} X_{i, j, t}+\beta_{2} z_{i, j, t} *$ Foreignbank $_{i, t}+\beta_{3}$ Foreignbank $_{j}+\beta_{4}$ Country $_{i}+\varepsilon_{i, j, t}$

where $X_{i, j, t}$ is a vector of all the explanatory variables in the benchmark model except for the variable $z_{i, j, t}$ which is the variable that interacts with the foreign bank dummy in each alternative specification. This specification is used to test if indigenous and foreign banks' NPLs react similarly to changes in the macroeconomic environment and other indicators of bank performance.

\section{The results (Table 2) suggest that the determinants of NPLs are broadly comparable} across indigenous and foreign banks, with some differences. In Table 2, the interaction terms are highlighted in red; the top coefficient refers to indigenous banks and the bottom coefficient to foreign banks. The results suggest that the asset quality of foreign banks is more responsive to macroeconomic developments and, similarly, banks' profitability. For foreign banks, higher profitability reduces NPLs, while, for indigenous banks, there appears to be no link between bank profitability and asset quality. Indeed, the point estimates for ROA are statistically different for the two types of banks (Table 3). ${ }^{10}$ The results also suggest that foreign banks' asset quality is more responsive to the concentration of lending to households and indigenous banks to the construction sector, consistent with the relatively higher concentration of each bank types lending to these sectors; however, the differences in the coefficients are not statistically significant (Table 3).

\footnotetext{
${ }^{10}$ Table 3 presents the same specifications as in Table 2, except that instead of estimating the coefficient for each type of bank, we estimate the coefficient for the base bank (indigenous) and the coefficient for the difference between indigenous and foreign banks (the interaction term). The advantage of this presentation is that it shows if the difference in coefficients between the two banks is statistically different.
} 


\section{The Dynamics of Non-Performing Loans and Macroeconomic Effects}

11. This section assesses feedback effects between the banking sector through NPLs and the real economy using a panel VAR approach. The estimations are based on the following model:

$$
\begin{array}{r}
Y_{i t}=Y_{i t-1} A_{1}+Y_{i t-2} A_{2}+\cdots+Y_{i t-p+1} A_{p-1}+Y_{i t-p} A_{p}+X_{i t} B+\boldsymbol{u}_{\boldsymbol{i}}+\boldsymbol{e}_{i t}, \\
Y_{i t}=\left[\Delta \operatorname{snpl}_{i, t}, \Delta \operatorname{credit}_{i, t}, \Delta F D I_{i, t}, \Delta G D P_{i, t}, \Delta C P I_{i, t}\right]
\end{array}
$$

where $Y_{i, t}$ is a vector of five endogenous variables; $\Delta$ npli,t denotes growth in NPLs of the overall banking system in country $i$ in year $t ; \Delta$ crediti, denotes growth in credit to the private sector in country $i$ in year $t ; \Delta \mathrm{FDI}$, denotes growth in foreign direct investment in country $i$ in year $t ; \Delta \mathrm{GDP}_{\mathrm{i}, \mathrm{t}}$ denotes real GDP growth in country $i$ in year $t$; and $\triangle C P \mathrm{I}_{i, t}$ - average annual CPI inflation in percent. ${ }^{11} X_{i, t}$ is a vector of exogenous covariates; $u_{i}$ and $e_{i t}$ are vectors of dependent variablespecific panel fixed effects and idiosyncratic errors, respectively.

12. To take into account the close linkages between the ECCU and advanced economies, the model includes global macroeconomic variables as exogenous variables. Given that both global and domestic macroeconomic developments are found to be important determinants of NPLs and to capture the strong spillovers between advanced economies and the ECCU the model also includes advanced economies' real GDP growth as an exogenous variable. FDI is also added to account for the strong linkages between foreign direct investment inflows and economic performance in the ECCU. Given the region's susceptibility to natural disasters, a dummy variable to capture the impact of natural disasters is also included as an exogenous variable. ${ }^{12}$ Results are broadly robust to alternative ordering of variables.

\section{The model is estimated for a panel data set of the $\mathbf{6}$ independent ECCU economies} over the period 1996 to $2015 .{ }^{13}$ The panel is estimated on an annual frequency given that the major macroeconomic indicators are unavailable in higher frequency. To assess whether the strength of the feedback effects from the banking system to the real economy varies depending on the industry considered, quarterly models are also estimated using industry specific data on NPLS and credit growth.

\section{Quarterly models were estimated separately for personal, tourism, agriculture,} manufacturing, construction, and trade industries. The models are based on 2004Q1-2015Q4 period and include NPLs growth by sector, credit growth by sector, real GDP growth, and CPI inflation as endogenous variables; real GDP growth of advanced economies and natural disasters

\footnotetext{
${ }^{11}$ Two different specifications were considered for the definition of NPLs: the annual percentage change in nonperforming loans and the first difference of the ratio of NPL-to-total loans ratios-to-total loans. While qualitatively both specifications largely generate similar results, the former specification was used for more intuitive interpretation.

12 The dummy variable takes the value of one for the year in which a disaster occurred and zero otherwise.

${ }^{13}$ For a total of 120 observations. The data are aggregated by country.
} 
dummy as exogenous variables. Quarterly real GDP estimates are imputed using annual real GDP for the ECCU economies and quarterly real GDP data for the United States, given the close correlation of real GDP for the ECCU and the US.

\section{Panel VAR Impulse Response Functions suggest that a shock to NPL growth has} implications for economic activity and the credit cycle (Figure 1). A deterioration in asset quality has a negative effect on real GDP growth, CPI inflation, and FDI growth, but the results of the aggregate model are statistically significant only for the latter. Complementary sector-specific models, however, suggest that lower NPLs in the agricultural and construction sectors may result in a significant positive effect on real GDP growth. The aggregate model also suggests that a deterioration in asset quality leads to a decline in credit, although again the response is not statistically significant. The quarterly model results suggest that deterioration in asset quality leads to a statistically significant decline in credit to the more productive sectors of the economy, such as tourism, agriculture, construction, and manufacturing industries, meanwhile this negative relationship is much weaker for personal loans and trade industry.

\section{Macroeconomic performance also has a significant effect on asset quality. Stronger} economic performance leads to a statistically significant decline in NPL growth. This result, however, appears to be driven largely by the personal and tourism industries, which broadly comprise the majority of NPLs. Stronger economic activity also has a positive, however statistically insignificant, effect on FDI and credit growth. In the aggregate model, an increase in credit has a positive but statistically insignificant effect on economic activity. In the quarterly models, however, the effect is statistically significant for the agricultural and construction industries. The persistent nature of credit growth also implies that a shock to credit growth in the initial period lingers longer than in the case of other variables.

\section{Panel VAR variance decomposition also suggests important implications of economic} activity on NPL growth. Using variance decomposition of the panel VAR approach, we also assess the extent to which the forecast error variance of one variable is associated with exogenous shocks to other endogenous variables. The results indicate that over a 5-year horizon about 7 percent of NPLs is explained by economic performance variables, suggesting the importance of economic indicators to the banking sector health. Meanwhile, the effect of NPLs on economic performance is somewhat lower, as NPL growth explains about 1 percent of real GDP growth over the medium term.

\section{Conclusion}

18. The deterioration in asset quality experienced in the ECCU over the last decade is attributable to both macroeconomic conditions and bank-specific factors. For the small open economies of the ECCU, both domestic and global macroeconomic conditions directly affect banks' asset quality. NPLs are also sensitive to bank level factors. In particular, more profitable banks and banks with lower exposure to the volatile construction and tourism sectors and household loans tend to have lower NPLs. There is also some evidence that foreign owned banks systematically have 
lower NPLs than indigenous banks, suggesting that there are some important institutional differences across banks that affect their due diligence or risk management practices and/or NPL recovery strategies that have a meaningful impact on asset quality. These differences may also reflect the scale of banks' operations. Indigenous banks operate on a very small scale in their country of origin, while the foreign banks are primarily branches and subsidiaries of large Canadian banks with global operations. However, more detailed data on differences in institutional practices is needed to better understand whether and how the differences in the determinants of NPLs are driven by type of bank ownership.

\section{The assessment of the feedback effects emphasizes the strength of macro-financial}

feedback loops in the ECCU. The results indicate that a deterioration of asset quality leads to declining credit, with wide variations in the impact by sector. For the tourism, agriculture, manufacturing, and construction industries, a deterioration of asset quality leads to a significant and prolonged decline in credit growth. The results also suggest that an improvement in asset quality has the potential to boost real GDP growth in the ECCU, particularly through the agricultural and construction industries. In turn, real GDP growth also affects growth in NPLs as higher GDP growth likely through the associated lower unemployment and increase in disposable income, can significantly reduce loan delinquency rates, notably in personal and tourism sectors. However, the results also illustrate that FDI plays an important role in moderating the strength of macro-financial feedback loops in the ECCU. Indeed, the results highlight the importance of FDI financing for the region through its strong positive effect on economic growth, which is considerably stronger than that of domestic credit. 


\begin{tabular}{|c|c|c|c|c|c|c|c|c|}
\hline \multicolumn{9}{|c|}{ Table 1: Determinants of NPLs in the ECCU 1/ } \\
\hline & (1) & (2) & (3) & (4) & (5) & (6) & (7) & (8) \\
\hline & RE & RE & $\mathrm{FE}$ & $\mathrm{FE}$ & $\mathrm{RE}$ & $\mathrm{RE}$ & $\mathrm{FE}$ & $\mathrm{FE}$ \\
\hline \multirow[t]{2}{*}{$N P L s_{t-1}$} & $0.679 * * *$ & $0.662^{* * *}$ & $0.665^{* * *}$ & $0.639 * * *$ & $0.662^{* * *}$ & $0.651^{* * *}$ & $0.643^{* * *}$ & $0.626^{* * *}$ \\
\hline & $(0.019)$ & $(0.019)$ & $(0.020)$ & $(0.020)$ & $(0.020)$ & $(0.020)$ & $(0.020)$ & $(0.020)$ \\
\hline \multirow[t]{2}{*}{$N P L s_{t-2}$} & $0.207^{* * *}$ & $0.202^{* * *}$ & $0.197^{* * *}$ & $0.186^{* * *}$ & $0.200 * * *$ & $0.198^{* * *}$ & $0.187^{* * *}$ & $0.179 * * *$ \\
\hline & $(0.019)$ & $(0.019)$ & (0.019) & $(0.019)$ & $(0.019)$ & (0.019) & $(0.019)$ & $(0.019)$ \\
\hline \multirow[t]{2}{*}{ Advanced Economy Growth $\mathrm{t}_{\mathrm{t} 2}$} & $-0.010 * *$ & 0.000 & $-0.010 * *$ & 0.002 & $-0.008^{* *}$ & 0.000 & $-0.009 * *$ & 0.002 \\
\hline & $(0.004)$ & $(0.004)$ & $(0.004)$ & $(0.004)$ & $(0.004)$ & $(0.004)$ & $(0.004)$ & $(0.004)$ \\
\hline \multirow[t]{2}{*}{ Tourism Growth $\mathrm{t}-2$} & $0.002^{* * *}$ & $0.003^{* * *}$ & $0.002^{* * *}$ & $0.003^{* * *}$ & $0.002^{* * *}$ & $0.003^{* * *}$ & $0.002^{* * *}$ & $0.003^{* * *}$ \\
\hline & $(0.001)$ & $(0.001)$ & $(0.001)$ & $(0.001)$ & $(0.001)$ & $(0.001)$ & $(0.001)$ & $(0.001)$ \\
\hline \multirow{2}{*}{ Real Loan Growth $\mathrm{t}_{\mathrm{t}-1}$} & & & & & $-0.003^{* * *}$ & $-0.002^{* * *}$ & $-0.004^{* * *}$ & $-0.003^{* * *}$ \\
\hline & & & & & $(0.001)$ & $(0.001)$ & $(0.001)$ & $(0.001)$ \\
\hline \multirow{2}{*}{ Return on Assets $\mathrm{t}_{-1}$} & & & & & $-0.030 * *$ & -0.020 & -0.023 & -0.008 \\
\hline & & & & & $(0.013)$ & $(0.013)$ & $(0.014)$ & $(0.014)$ \\
\hline \multirow{2}{*}{ Household Loans/Total Loans $\mathrm{t}_{-1}$} & & & & & $0.030^{*}$ & 0.018 & $0.097^{* * *}$ & $0.076 * * *$ \\
\hline & & & & & $(0.016)$ & $(0.016)$ & $(0.028)$ & $(0.028)$ \\
\hline \multicolumn{9}{|l|}{ Construction and Tourism Loans/Total } \\
\hline \multirow[t]{2}{*}{ Loans $_{\mathrm{t}-1}$} & & & & & $0.022^{*}$ & 0.011 & $0.035^{* *}$ & 0.009 \\
\hline & & & & & $(0.012)$ & $(0.012)$ & $(0.016)$ & $(0.016)$ \\
\hline \multirow[t]{2}{*}{ Foreign bank's dummy } & $-0.072 * * *$ & $-0.086 * * *$ & & & $-0.103^{* * *}$ & $-0.110 * * *$ & & \\
\hline & $(0.020)$ & $(0.020)$ & & & $(0.022)$ & $(0.022)$ & & \\
\hline \multirow[t]{2}{*}{ Dominica dummy } & 0.003 & 0.013 & & & -0.006 & 0.000 & & \\
\hline & $(0.031)$ & $(0.030)$ & & & $(0.031)$ & $(0.031)$ & & \\
\hline \multirow[t]{2}{*}{ Grenada dummy } & $-0.050^{*}$ & $-0.052^{*}$ & & & $-0.069 * *$ & $-0.069 * *$ & & \\
\hline & $(0.030)$ & $(0.029)$ & & & $(0.030)$ & $(0.030)$ & & \\
\hline \multirow[t]{2}{*}{ St. Kitts and Nevis dummy } & $-0.048^{*}$ & $-0.054^{*}$ & & & $-0.067^{* *}$ & $-0.072^{* *}$ & & \\
\hline & $(0.028)$ & $(0.028)$ & & & $(0.029)$ & $(0.028)$ & & \\
\hline \multirow[t]{2}{*}{ St. Lucia dummy } & 0.034 & 0.046 & & & 0.029 & 0.039 & & \\
\hline & $(0.028)$ & $(0.028)$ & & & $(0.029)$ & $(0.029)$ & & \\
\hline \multirow[t]{2}{*}{ St.Vincent and the Grenadines dummy } & -0.038 & -0.042 & & & -0.052 & $-0.057 *$ & & \\
\hline & $(0.032)$ & $(0.031)$ & & & $(0.033)$ & $(0.033)$ & & \\
\hline \multirow[t]{2}{*}{ Time dummy } & & $0.130 * * *$ & & $0.170^{* * *}$ & & $0.116^{* * *}$ & & $0.152^{* * *}$ \\
\hline & & $(0.021)$ & & $(0.022)$ & & $(0.022)$ & & $(0.023)$ \\
\hline \multirow[t]{2}{*}{ Constant } & $-0.194 * * *$ & $-0.312 * * *$ & $-0.320^{* * *}$ & $-0.508^{* * *}$ & $-0.150^{* * *}$ & $-0.275^{* * *}$ & $-0.297 * * *$ & $-0.512^{* * *}$ \\
\hline & $(0.030)$ & $(0.035)$ & $(0.030)$ & $(0.038)$ & $(0.041)$ & $(0.047)$ & $(0.047)$ & $(0.057)$ \\
\hline Observations & 2,415 & 2,415 & 2,415 & 2,415 & 2,384 & 2,384 & 2,384 & 2,384 \\
\hline Number of Bank_code & 35 & 35 & 35 & 35 & 34 & 34 & 34 & 34 \\
\hline \multirow{2}{*}{\multicolumn{9}{|c|}{ Standard errors in parentheses }} \\
\hline${ }^{* * *} p<0.01,{ }^{* *} p<0.05, * p<0.1$ & & & & & & & & \\
\hline \multicolumn{9}{|c|}{$\begin{array}{l}\text { 1./ The dependent variable is the logit transformation of the NPL ratio for bank } i \text { in country } j \text { at time } t \text {. RE and FE indicate } \\
\text { random and fixed effects estimation, respectively. }\end{array}$} \\
\hline
\end{tabular}


Table 2: Determinants of NPLs in the ECCU: Differences Between Indigenous and Foreign Banks 1/

\begin{tabular}{|c|c|c|c|c|c|c|c|}
\hline & (1) & $(2)$ & (3) & (4) & (5) & (6) & (7) \\
\hline$N P L s_{t-1}$ & $\begin{array}{c}0.662^{* * *} \\
(0.020)\end{array}$ & $\begin{array}{c}0.663^{* * *} \\
(0.020)\end{array}$ & $\begin{array}{c}0.662^{* * *} \\
(0.020)\end{array}$ & $\begin{array}{c}0.662^{* * *} \\
(0.020)\end{array}$ & $\begin{array}{c}0.655^{* * *} \\
(0.020)\end{array}$ & $\begin{array}{c}0.662 * * * \\
(0.020)\end{array}$ & $\begin{array}{c}0.662 * * * \\
(0.020)\end{array}$ \\
\hline$N P L s_{t-2}$ & $\begin{array}{c}0.200^{* * *} \\
(0.019)\end{array}$ & $\begin{array}{c}0.200 * * * \\
(0.019)\end{array}$ & $\begin{array}{c}0.201^{* * *} \\
(0.019)\end{array}$ & $\begin{array}{c}0.200^{* * *} \\
(0.019)\end{array}$ & $\begin{array}{c}0.199 * * * \\
(0.019)\end{array}$ & $\begin{array}{c}0.201^{* * *} \\
(0.019)\end{array}$ & $\begin{array}{c}0.200 * * * \\
(0.019)\end{array}$ \\
\hline Advanced Economy Growth ${ }_{\mathrm{t}-2}$ & $\begin{array}{c}-0.008^{* *} \\
(0.004)\end{array}$ & $\begin{array}{l}-0.006 \\
(0.007)\end{array}$ & $\begin{array}{c}-0.008^{* *} \\
(0.004)\end{array}$ & $\begin{array}{c}-0.008^{*} \\
(0.004)\end{array}$ & $\begin{array}{c}-0.007^{*} \\
(0.004)\end{array}$ & $\begin{array}{c}-0.008^{*} \\
(0.004)\end{array}$ & $\begin{array}{c}-0.008 * * \\
(0.004)\end{array}$ \\
\hline Foreign banks & & $\begin{array}{c}-0.009 * \\
(0.005)\end{array}$ & & & & & \\
\hline Tourism Growth $_{\mathrm{t}-2}$ & $\begin{array}{c}0.002^{* * *} \\
(0.001)\end{array}$ & $\begin{array}{c}0.002^{* * *} \\
(0.001)\end{array}$ & $\begin{array}{c}0.002 \\
(0.001)\end{array}$ & $\begin{array}{c}0.002^{* * *} \\
(0.001)\end{array}$ & $\begin{array}{c}0.002^{* * *} \\
(0.001)\end{array}$ & $\begin{array}{c}0.002^{* * *} \\
(0.001)\end{array}$ & $\begin{array}{c}0.002 * * * \\
(0.001)\end{array}$ \\
\hline Foreign banks & & & $\begin{array}{c}0.002^{* * *} \\
(0.001)\end{array}$ & & & & \\
\hline Real Loan Growth ${ }_{\mathrm{t}-1}$ & $\begin{array}{c}-0.003^{* * *} \\
(0.001)\end{array}$ & $\begin{array}{c}-0.003^{* * *} \\
(0.001)\end{array}$ & $\begin{array}{c}-0.003^{* * *} \\
(0.001)\end{array}$ & $\begin{array}{c}-0.003^{* *} \\
(0.001)\end{array}$ & $\begin{array}{c}-0.003^{* * *} \\
(0.001)\end{array}$ & $\begin{array}{c}-0.003^{* * *} \\
(0.001)\end{array}$ & $\begin{array}{c}-0.003^{* * *} \\
(0.001)\end{array}$ \\
\hline Foreign banks & & & & $\begin{array}{c}-0.003^{* * *} \\
(0.001)\end{array}$ & & & \\
\hline Return on Assets $\mathrm{t}_{\mathrm{t}-1}$ & $\begin{array}{c}-0.030 * * \\
(0.013)\end{array}$ & $\begin{array}{c}-0.030 * * \\
(0.013)\end{array}$ & $\begin{array}{c}-0.030 * * \\
(0.013)\end{array}$ & $\begin{array}{c}-0.030 * * \\
(0.013)\end{array}$ & $\begin{array}{c}0.026 \\
(0.019)\end{array}$ & $\begin{array}{c}-0.031^{* *} \\
(0.013)\end{array}$ & $\begin{array}{c}-0.030 * * \\
(0.013)\end{array}$ \\
\hline Foreign banks & & & & & $\begin{array}{c}-0.085^{* * *} \\
(0.019)\end{array}$ & & \\
\hline Household Loans/Total Loans ${ }_{\mathrm{t}-1}$ & $\begin{array}{c}0.030 * \\
(0.016)\end{array}$ & $\begin{array}{c}0.030 * \\
(0.016)\end{array}$ & $\begin{array}{c}0.030 * \\
(0.016)\end{array}$ & $\begin{array}{c}0.030 * \\
(0.016)\end{array}$ & $\begin{array}{c}0.038 * * \\
(0.016)\end{array}$ & $\begin{array}{c}0.016 \\
(0.023)\end{array}$ & $\begin{array}{c}0.031^{* *} \\
(0.016)\end{array}$ \\
\hline Foreign banks & & & & & & $\begin{array}{c}0.040 * * \\
(0.020)\end{array}$ & \\
\hline \multicolumn{8}{|l|}{ Construction and Tourism Loans/Total } \\
\hline Loans $_{\mathrm{t}-1}$ & $\begin{array}{l}0.022 * \\
(0.012)\end{array}$ & $\begin{array}{c}0.022 * \\
(0.012)\end{array}$ & $\begin{array}{l}0.022 * \\
(0.012)\end{array}$ & $\begin{array}{l}0.022 * \\
(0.012)\end{array}$ & $\begin{array}{c}0.026 * * \\
(0.012)\end{array}$ & $\begin{array}{c}0.023^{*} \\
(0.012)\end{array}$ & $\begin{array}{c}0.034 \\
(0.022)\end{array}$ \\
\hline Foreign banks & & & & & & & $\begin{array}{c}0.018 \\
(0.013)\end{array}$ \\
\hline Foreign bank's dummy & $\begin{array}{c}-0.103^{* * *} \\
(0.022)\end{array}$ & $\begin{array}{c}-0.096^{* * *} \\
(0.029)\end{array}$ & $\begin{array}{c}-0.105^{* * *} \\
(0.022)\end{array}$ & $\begin{array}{c}-0.101^{* * *} \\
(0.023)\end{array}$ & $\begin{array}{c}-0.071^{* * *} \\
(0.023)\end{array}$ & $\begin{array}{c}-0.099 * * * \\
(0.023)\end{array}$ & $\begin{array}{c}-0.139 * * \\
(0.057)\end{array}$ \\
\hline Dominica dummy & $\begin{array}{c}-0.006 \\
(0.031)\end{array}$ & $\begin{array}{l}-0.006 \\
(0.031)\end{array}$ & $\begin{array}{c}-0.006 \\
(0.031)\end{array}$ & $\begin{array}{c}-0.006 \\
(0.031)\end{array}$ & $\begin{array}{c}-0.005 \\
(0.031)\end{array}$ & $\begin{array}{l}-0.007 \\
(0.031)\end{array}$ & $\begin{array}{c}-0.007 \\
(0.031)\end{array}$ \\
\hline Grenada dummy & $\begin{array}{c}-0.069 * * \\
(0.030)\end{array}$ & $\begin{array}{c}-0.069 * * \\
(0.030)\end{array}$ & $\begin{array}{c}-0.069 * * \\
(0.030)\end{array}$ & $\begin{array}{c}-0.069^{* *} \\
(0.030)\end{array}$ & $\begin{array}{c}-0.080^{* * *} \\
(0.030)\end{array}$ & $\begin{array}{c}-0.064^{* *} \\
(0.031)\end{array}$ & $\begin{array}{c}-0.067^{* *} \\
(0.031)\end{array}$ \\
\hline St. Kitts and Nevis dummy & $\begin{array}{c}-0.067^{* *} \\
(0.029)\end{array}$ & $\begin{array}{c}-0.067^{* *} \\
(0.029)\end{array}$ & $\begin{array}{c}-0.067^{* *} \\
(0.029)\end{array}$ & $\begin{array}{c}-0.067^{* *} \\
(0.029)\end{array}$ & $\begin{array}{c}-0.074^{* * *} \\
(0.029)\end{array}$ & $\begin{array}{c}-0.068^{* *} \\
(0.029)\end{array}$ & $\begin{array}{c}-0.067^{* *} \\
(0.029)\end{array}$ \\
\hline St. Lucia dummy & $\begin{array}{c}0.029 \\
(0.029)\end{array}$ & $\begin{array}{c}0.029 \\
(0.029)\end{array}$ & $\begin{array}{c}0.029 \\
(0.029)\end{array}$ & $\begin{array}{c}0.029 \\
(0.029)\end{array}$ & $\begin{array}{c}0.024 \\
(0.029)\end{array}$ & $\begin{array}{c}0.035 \\
(0.030)\end{array}$ & $\begin{array}{c}0.030 \\
(0.029)\end{array}$ \\
\hline St.Vincent and the Grenadines dummy & $\begin{array}{l}-0.052 \\
(0.033)\end{array}$ & $\begin{array}{l}-0.052 \\
(0.033)\end{array}$ & $\begin{array}{l}-0.052 \\
(0.033)\end{array}$ & $\begin{array}{l}-0.052 \\
(0.033)\end{array}$ & $\begin{array}{l}-0.059 * \\
(0.033)\end{array}$ & $\begin{array}{l}-0.048 \\
(0.033)\end{array}$ & $\begin{array}{l}-0.052 \\
(0.033)\end{array}$ \\
\hline Constant & $\begin{array}{c}-0.150^{* * *} \\
(0.041)\end{array}$ & $\begin{array}{c}-0.154^{* * *} \\
(0.043)\end{array}$ & $\begin{array}{c}-0.148^{* * *} \\
(0.041)\end{array}$ & $\begin{array}{c}-0.151^{* * *} \\
(0.041)\end{array}$ & $\begin{array}{c}-0.171^{* * *} \\
(0.041)\end{array}$ & $\begin{array}{c}-0.155^{* * *} \\
(0.042)\end{array}$ & $\begin{array}{c}-0.125^{* *} \\
(0.055)\end{array}$ \\
\hline Observations & 2,384 & 2,384 & 2,384 & 2,384 & 2,384 & 2,384 & 2,384 \\
\hline Number of Bank_code & 34 & 34 & 34 & 34 & 34 & 34 & 34 \\
\hline
\end{tabular}

Standard errors in parentheses

$* * * \mathrm{p}<0.01, * * \mathrm{p}<0.05, * \mathrm{p}<0.1$

1./ The dependent variable is the logit transformation of the NPL ratio for bank $i$ in country $j$ at time $t$. Random effects panel estimation. In each equation where an interaction term is present the value of the coefficient is for the indigenous banks, unless it clearly states it is for foreign banks. 
Table 3: Determinants of NPLs: Differences Between Indigenous and Foreign Banks 1/

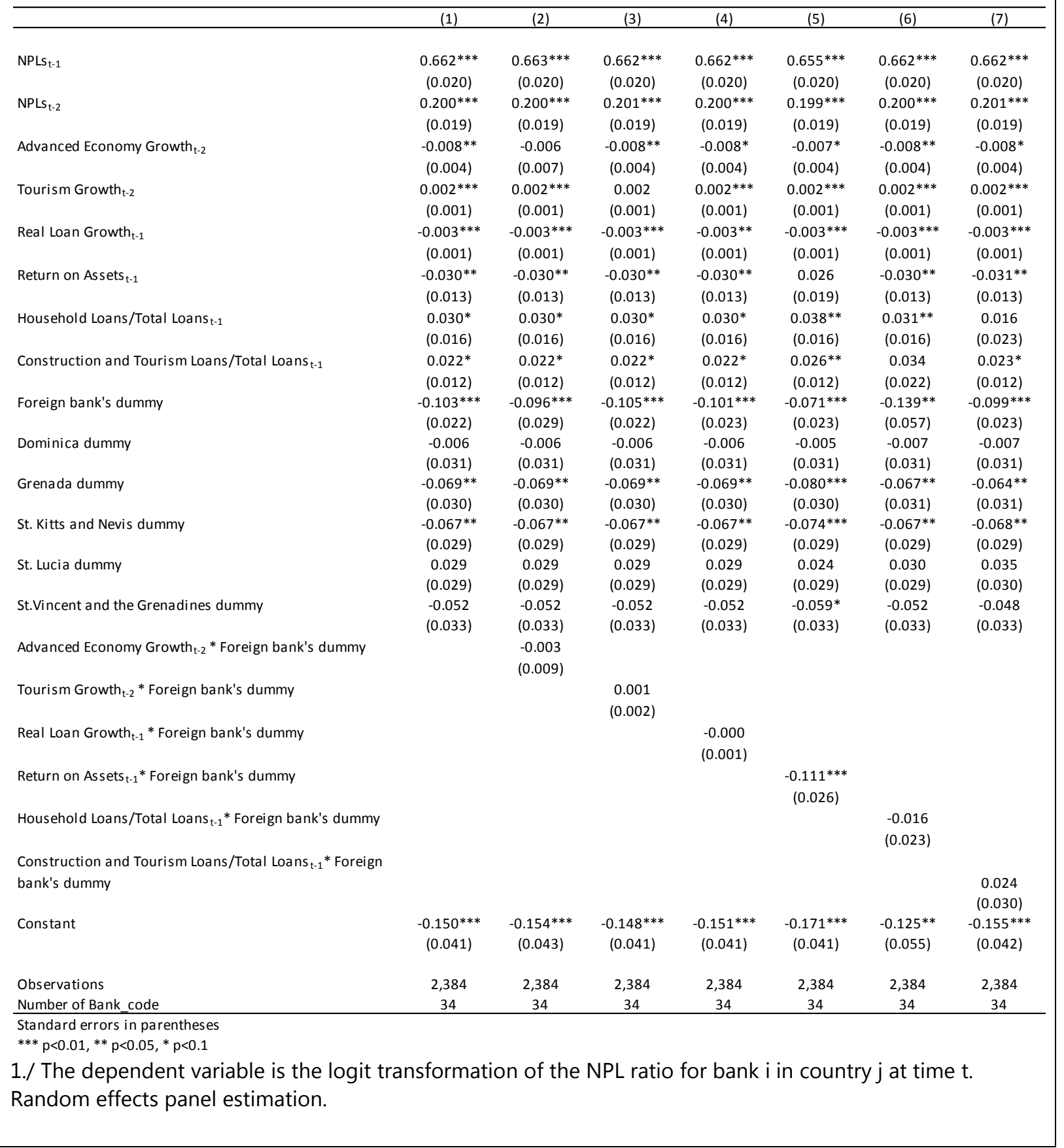




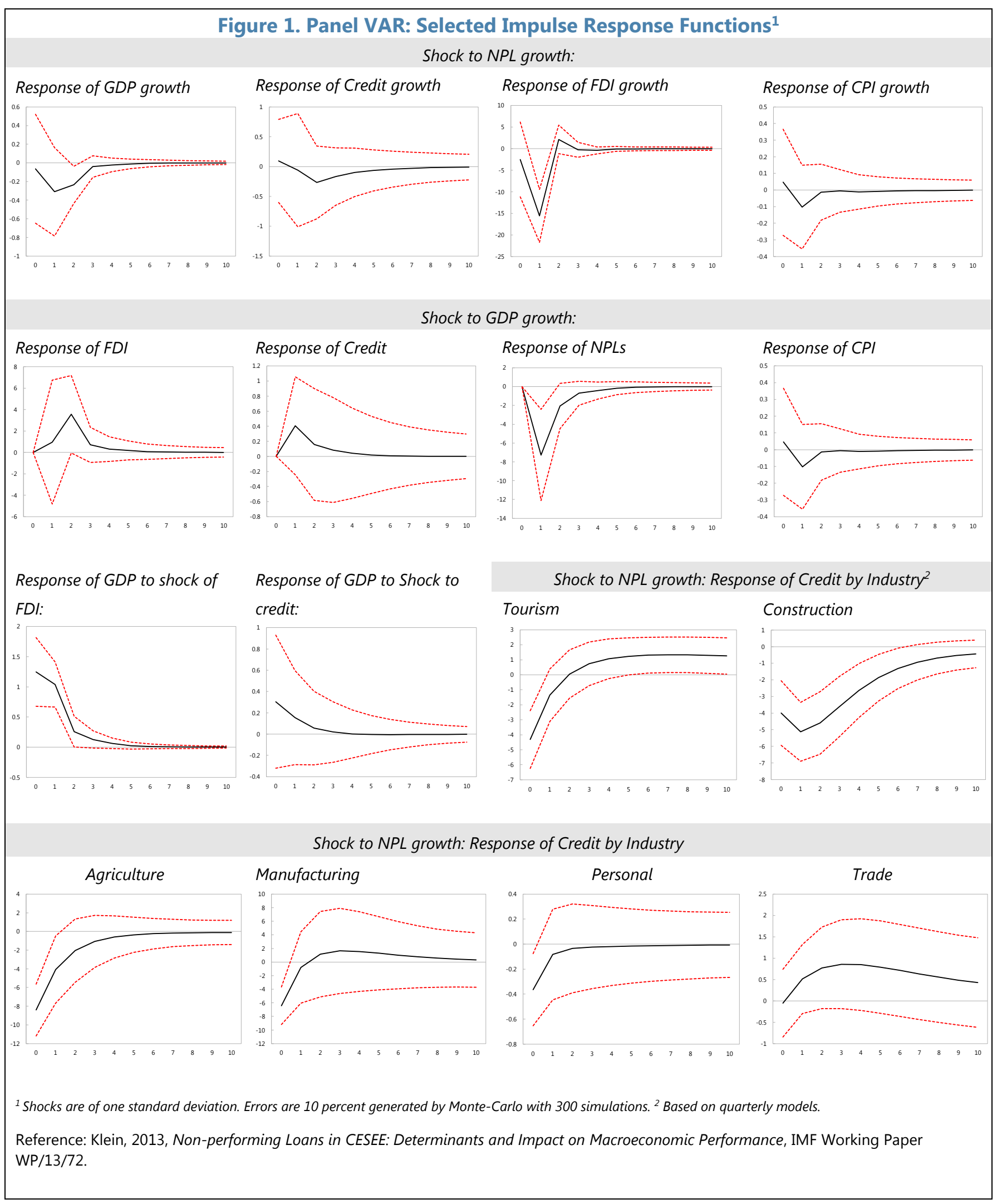




\section{Annex VI. Impact of the Withdrawal of Correspondent Banking Relationships (CBRs) on the ECCU1}

1. Faced with the prospect of sizeable fines from their regulators for AML/CFT violations, international banks have sought to limit their exposure to the perceived risk posed by certain customers or partners, a practice that has come to be known as "de-risking". Stricter regulations, combined with increased fines, have raised the cost of compliance along with the consequences of errors. Thus, even though the probability of a fine may be low, many international banks decide to exit certain markets where there is no potential for profits that could match the possible fines they may be subject to. This behavior has typically manifested itself in these banks ceasing to provide correspondent banking and trade finance services to other banks or customers, typically located in developing countries. In a recent World Bank survey (2015), banking authorities in Latin America and the Caribbean report the largest decline of correspondent accounts, although respondents mentioned that, generally speaking, the trend is more apparent

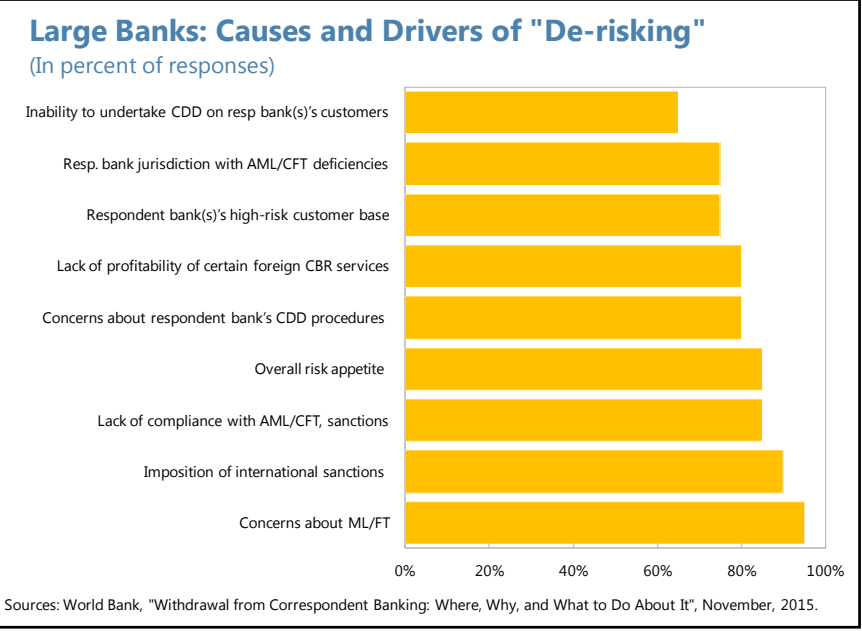
in the Caribbean than in Latin America.

2. The incidence of the withdrawal of CBRs appears to be negatively correlated with the size of the client institution, as smaller commercial banks find it more difficult to maintain CBRs given the low volume of their operations in relation to the perceived risk. Discussions with the ECCU banks and authorities also support this finding, as the low volume of correspondent banking operations is often cited as the leading cause of "de-risking" by global banks from ECCU.

\section{The growing occurrence of the withdrawal of CBRs from ECCU has prompted} discussions on possible options to address the consequences. In ECCU, substantial linkages with the United States, including through goods trade, tourism inflows, foreign direct investment, financial sector, as well as the remittances flows, among others, justify the need for USD transactions and correspondent banking relationships with foreign banks; all suggesting that the withdrawal of CBRs would impair the ability to conduct business in ECCU.

4. To assess the extent of the withdrawal of CBRs in the ECCU, staff conducted a survey of 10 indigenous onshore commercial banks and 5 regional offshore banking regulators. The survey results indicate that, while the loss of correspondent banking relationships (CBRs) has been

\footnotetext{
${ }^{1}$ Prepared by Alla Myrvoda.
} 
experienced in both the offshore and onshore banking sectors, the more severe consequences have been felt in the offshore sector.

\section{The sampled onshore indigenous banks together constitute about $\mathbf{5 5}$ percent of the}

locally incorporated banks' assets. Five banks reported either having lost a CBR in the last 2 years, or receiving an unofficial notification that a CBR termination may be forthcoming, with the low volume of operations given as the primary reason, although a reason is not always provided. While some banks reported difficulties establishing USD lines with other correspondent banks, most of the interviewed banks indicated that they either replaced the lost CBRs or in rare occasions did not actively seek a replacement given a sufficient number of other existing CBRs in place. Instead, the region has seen a significant increase in CBR fees for the onshore sector, which on average almost doubled, and in some cases tripled over the last 2 years. While the number of correspondent banking relationships tends to vary greatly by bank, depending on its business model, the median number of relationships currently stands at about four, some of which may be regional, however.

\section{Some banks have also reported terminating operations with certain types of} businesses, such as money services entities. Since foreign-owned banks reportedly are winnowing out seemingly riskier businesses from their operations, the burden of servicing such businesses has fallen increasingly on the indigenous banks, making them less attractive to their correspondent banks. However, some indigenous banks also reported discontinuing money services operations due to "de-risking" concerns.

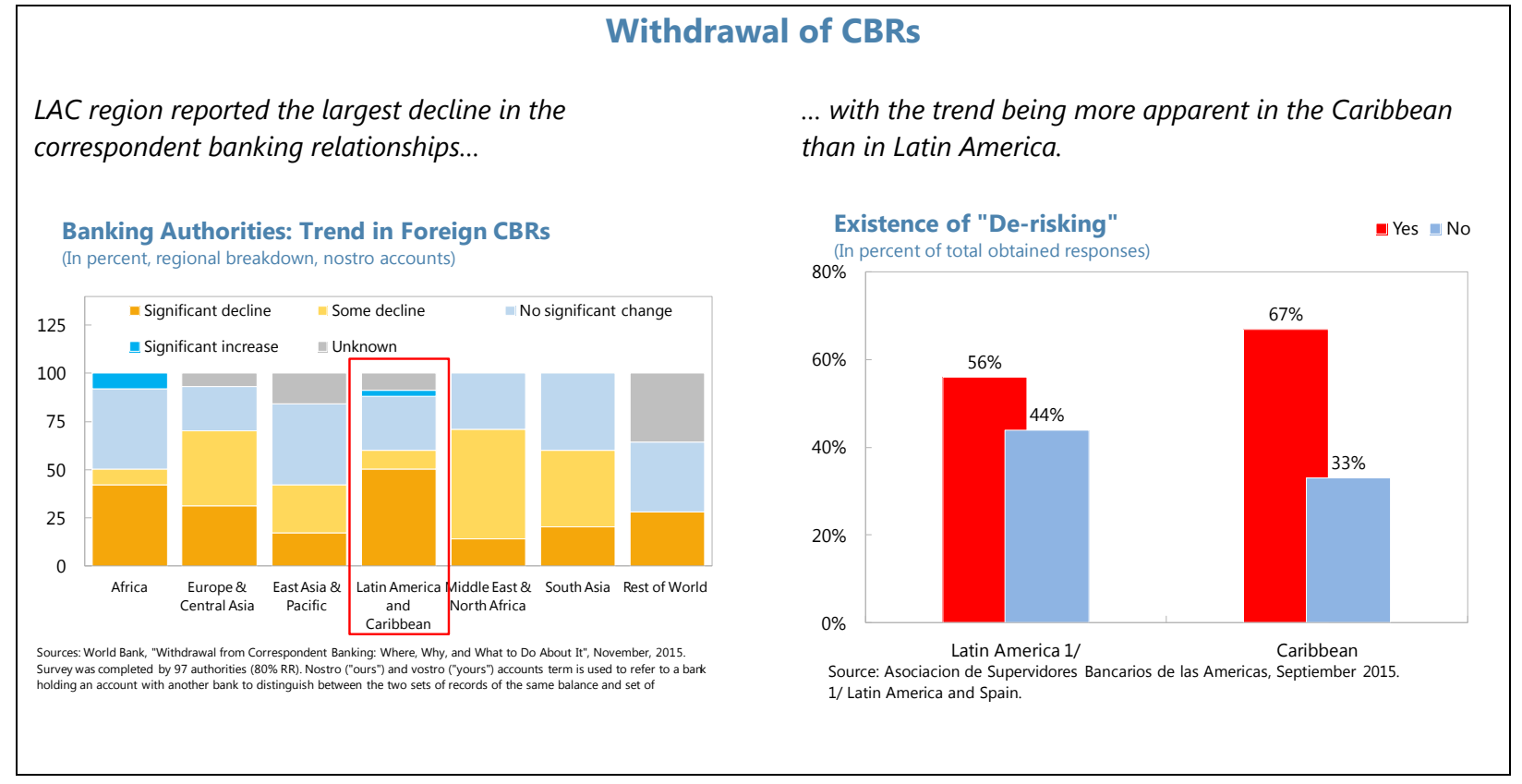

7. In the offshore banking sector, the difficulty of maintaining and obtaining CBRs has jeopardized the growth of the offshore banking. Authorities reported instances of offshore banks, unable to maintain or obtain correspondent banking relationships, expressing intentions to 
relocate to another jurisdiction where the issue of the withdrawal of CBRs is believed to be less severe, or terminating their license due to inability to establish a CBR. In addition, the number of new applications for the establishment of offshore banks is reported to have declined significantly over the last few years. Staff's survey of offshore banking regulators indicates that, while many offshore banks have been able to replace the lost CBRs, in some instances the prolonged search placed the sustainability

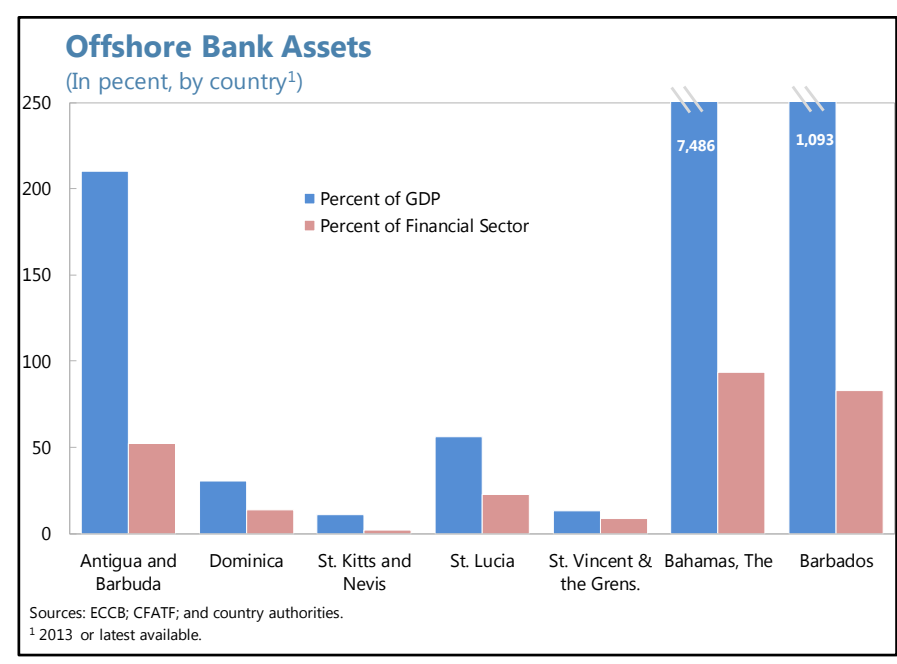
of the institution at risk. The trend has also severely restricted the pool of offshore banking license applications in the ECCU territories, as some jurisdictions have not received a viable offshore bank licensing application in years, while in some others, provisional licenses granted to offshore banks pending evidence of a CBR had to be revoked. According to the respondents, while small size is the main reason given for withdrawal from the region, correspondent banks also name pressures from the correspondent's own regulator, perceptions of the Caribbean as having high risk, and cost efficiency incompatible with the compliance costs, among the main reasons for terminating CBRs.

\section{CARICOM is attempting to assess the issue and develop strategies to address the} effects of the withdrawal of CBRs. To lead the response, CARICOM has set up a Committee of Ministers of Finance on Correspondent Banking — chaired by Antigua and Barbuda's Prime Minister-that is also supported by leading regional institutions including the Committee of Central Bank Governors (CCBG), the Caribbean Association of Banks (CAB) and the Caribbean Financial Action Task Force (CFATF). Since low volume of correspondent banking transactions is often considered the main cause of the withdrawal of CBRs in ECCU, authorities believe that efforts to consolidate such operations into larger volumes would help alleviate the issue of small scale. Thus, while authorities have long discussed banking sector amalgamation in the context of addressing efficiency and profitability issues, the accelerated withdrawal of correspondent banks strengthens the case for the amalgamation. At the CARICOM level, the authorities are also exploring the feasibility and practicality establishing a Caribbean-owned non-deposit taking financial institution in the United States, through which banks from the Caribbean countries would make pre-vetted payments.

\section{At the same time, ECCU authorities are continuing to strengthen their regulatory} frameworks, including AML/CFT and international tax cooperation. All ECCU countries are committed to the international certification processes of the Financial Sector Assessment Program (FSAP), Caribbean Financial Action Task Force (CFATF), and the Global Forum, and the Foreign Account Tax Compliance Act (FATCA). The latest reports of the CFATF indicate that the ECCU economies have made significant progress toward compliance with FATF's 2003 AML/CFT recommendations over the last few years (Table 1 ). The next round of evaluations by CFATF will 
assess ECCU countries' compliance against the revised 2012 FATF standard and will include an evaluation of the effectiveness of their AML/CFT framework. According to the 2015 Report by the Global Forum on Transparency and Exchange of Information for Tax Purposes, all jurisdictions in ECCU have undergone phase 1 and 2 reviews, with the exception of Dominica, which has a undergone phase 1 review and whose legal framework was judged to be adequate to permit the commencement of phase 2 review in 2015. For the phase 2 countries, Grenada, Montserrat, St. Kitts and Nevis, and St. Vincent and the Grenadines were largely compliant with the requirements, while Anguilla, Antigua and Barbuda, and St. Lucia were classified as partially compliant (Table 2).

\section{The international policy community has made a range of recommendations to cushion} the effect of the withdrawal of CBRs. Supervisors should ensure that banks follow risk-based supervision, given that this approach requires institutions to determine the risk level of their customers and take appropriate action to reduce the risk. As such, the ECCU regulators of the banking (onshore and offshore) and non-banking sectors are currently working on a timely and effective implementation of risk-based supervision and the BASEL II framework. Supervisors and other authorities are also encouraged to ensure the effective implementation of international AML/CFT standards. A clear explanation of the extent of due diligence on the customer's customers (KYCC obligations) by supervisors would clarify banks' obligations. Meanwhile better information flow and sharing between the correspondent and respondent banks would limit uncertainty. Authorities should collect information on the evolving status of correspondent banking in their jurisdictions, as having more comprehensive statistics would aid in addressing the vulnerabilities at the global level.

\section{References}

Warden, S., "De-risking and its Consequences for Global Commerce and the Financial System", Milken Institute, Center for Financial Markets, July, 2015.

Warden, S., "Climate Fix?", Milken Institute Review, A Journal of Economic Policy, June, 2015.

World Bank, "Report on the G20 Survey on De-Risking Activities in the Remittance Market", October, 2015.

World Bank, "Withdrawal from Correspondent Banking: Where, Why, and What to Do About It", November, 2015. 


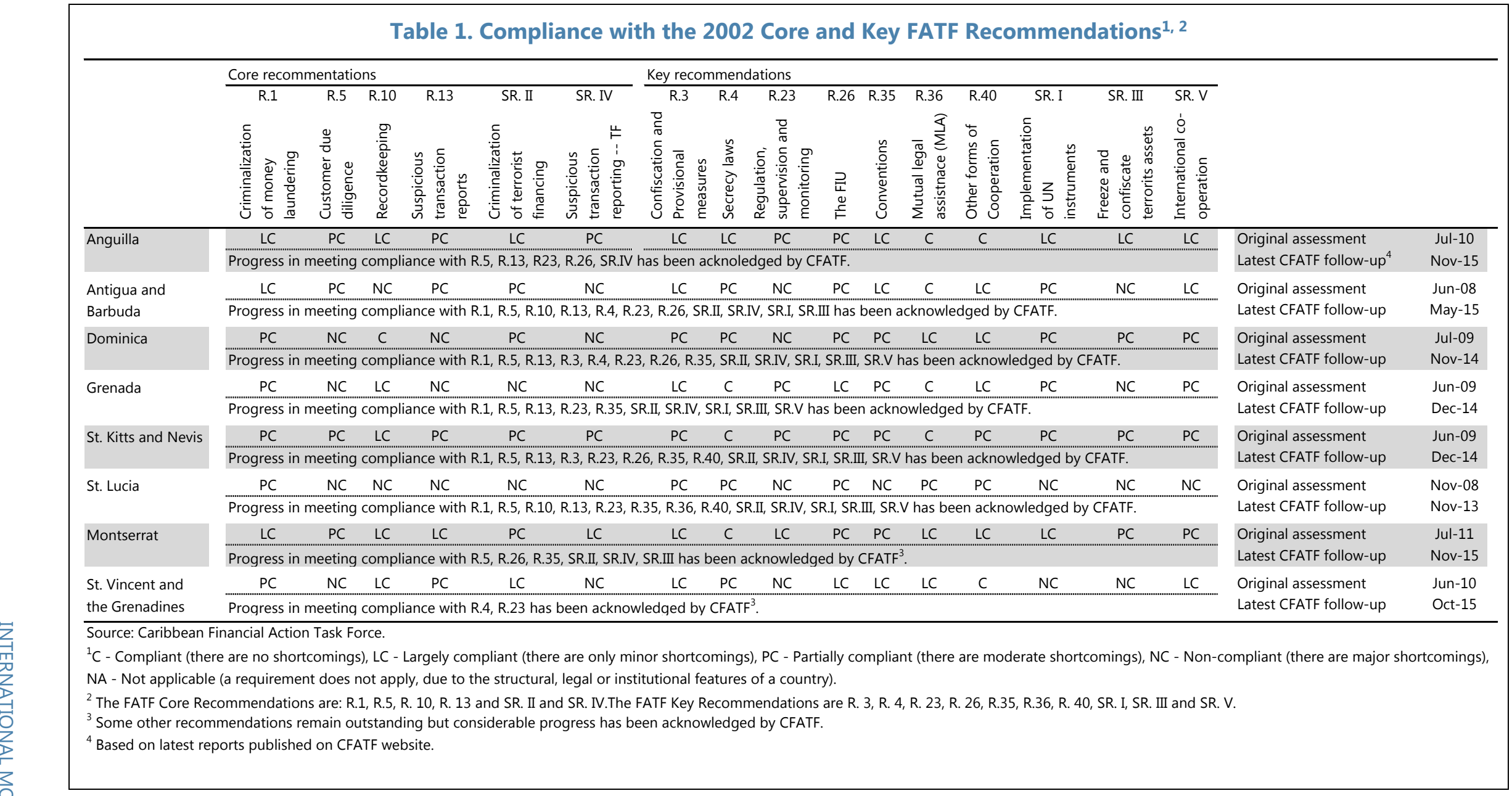




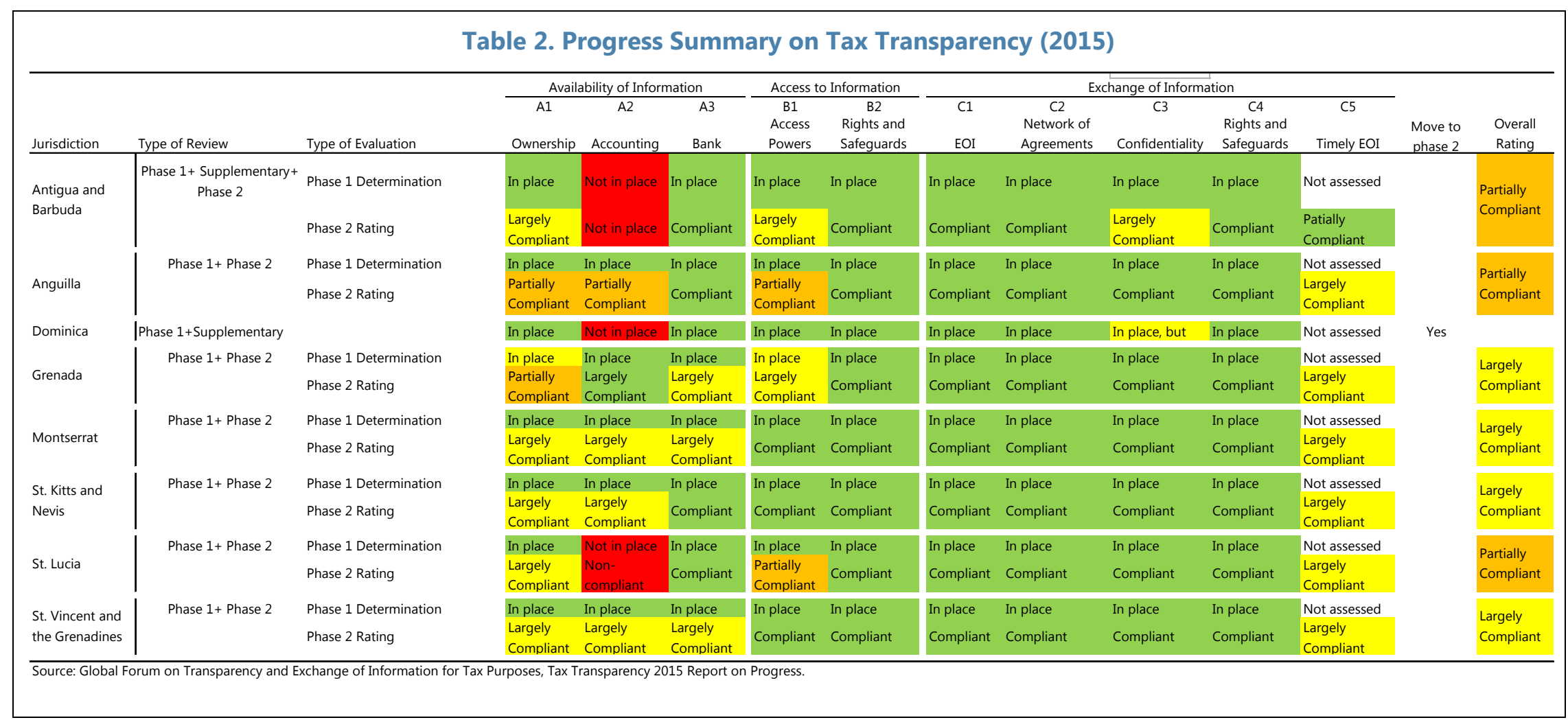




\title{
Annex VII. Public Financial Management in the ECCU: An Examination of PEFA Results ${ }^{1}$
}

\begin{abstract}
With a view to assessing the progress in PFM reform, CARTAC has undertaken a comparative review of PFM practices in ECCU member countries" against overall characteristics of a "core PFM" framework ${ }^{3}$ as defined in the IMF Good Practice Note (GPN) on 'Sequencing PFM Reform' (Diamond, 2013). This analysis has produced a number of interesting findings regarding the standard of PFM across the ECCU region.
\end{abstract}

While a number of reforms are still in progress, it is clear that the ECCU region has made strides in strengthening PFM systems. Overall, the results of PEFAs show a PFM system that is sound, but there are notable weaknesses in aspects of risk management, external scrutiny and transparency. There are also considerable variations in performance both overall and across the key PEFA dimensions. The ECCU region has met core PFM standards in: (1) policy based budgeting - where features of a strong multi-year approach are in place and reflected in a model budget calendar; and, (2) predictability and control in budget

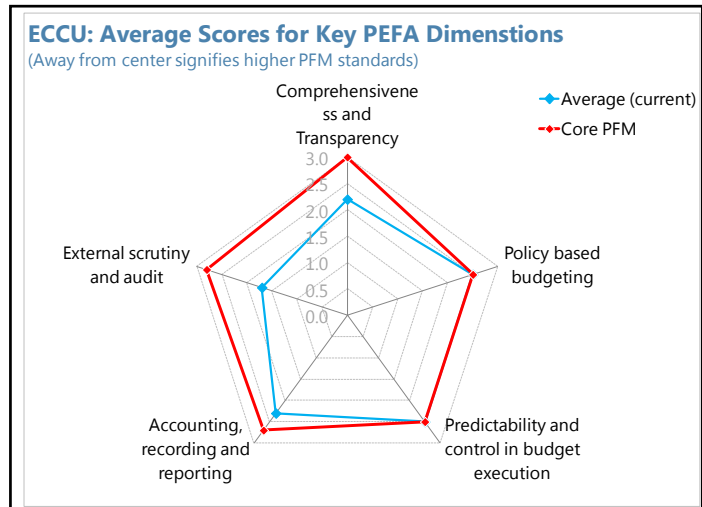
execution - where quality cash plans and commitment control enable effective management of the release of the budget. However, the frequent use of supplementary appropriations as the Budget year progresses offset the strength in this PEFA category. Generally, good information on budget execution is available to decision makers but publishing such data would greatly enhance transparency. Within tax administration, operational efficiency is compromised with the accumulation of tax arrears. Procurement was consistently rated poorly which potentially has adverse implications for the efficiency of service delivery.

PFM performance is weaker in more complex areas where reforms take longer to implement and more complex institutional and capacity is required. This is partially evident in accounting, recording and reporting, but particularly visible in the area of comprehensiveness and transparencywhere the lack of data available on unreported government operations (such as National Insurance Schemes, Citizen by Investment Programs) and fiscal risks, particularly those associated with state owned enterprises, are generally poor. The limited information available on the underlying economic parameters in the budget documentation also explains the weak scores in this category.

\footnotetext{
${ }^{1}$ Prepared by Matthew Smith (CARTAC).

${ }^{2}$ Analysis was derived from the PEFA assessments conducted from 2013-2016 in Anguilla, Antigua \& Barbuda, Dominica, Grenada, Montserrat and St Kitts \& Nevis. Dominica and St Kitts assessments while finalized are yet to be peer reviewed.

${ }^{3}$ As defined in the IMF Good Practice Note (GPN) on 'Sequencing PFM Reform' (Diamond, 2013).
} 
The inclusion of economic forecasts and a comprehensive fiscal strategy - including an assessment of fiscal risks, in the budget documentation would help address deficiencies in this dimension. Finally, the region as a whole suffers from poor external scrutiny and audit oversight - weaknesses in accountability mechanisms make external audits and their scrutiny ineffective as counter-checks on inefficient use of resources. 


\section{Annex VIII. Assessing Government Self-Insurance Needs against Natural Disasters: An Application to the ECCU1}

This paper presents estimates of the size and the annual budget contributions needed for the creation of saving funds (SF) to self-insure against natural disasters (ND) in the ECCU. The results indicate that ECCU countries would need SF stocks in the range of 6-12 percent of GDP and budget savings of 0.5-1.9 percent of GDP per year for the financial sustainability of the SFs with a low probability of depletion. The results are based on a Monte-Carlo experiment that simulates ND shocks and their impact on output and government finances.

\section{A. Introduction}

\section{Tropical storms and other forms of ND continue to affect the ECCU, resulting in} human loss, destruction of infrastructure, and fiscal costs. This annex proposes the creation of SF for ND financed with resources from the Citizenship by Investment programs (CIPs). With the exception of St. Vincent and the Grenadines, all independent ECCU countries have CIPs, which, in recent years, have generated a surge in budget revenues and become important from a macroeconomic perspective. ${ }^{2}$ If spent without regard to general macroeconomic conditions, CIPs can pose challenges to macroeconomic management, including on financial stability, fiscal discipline, external competitiveness, and growth (see

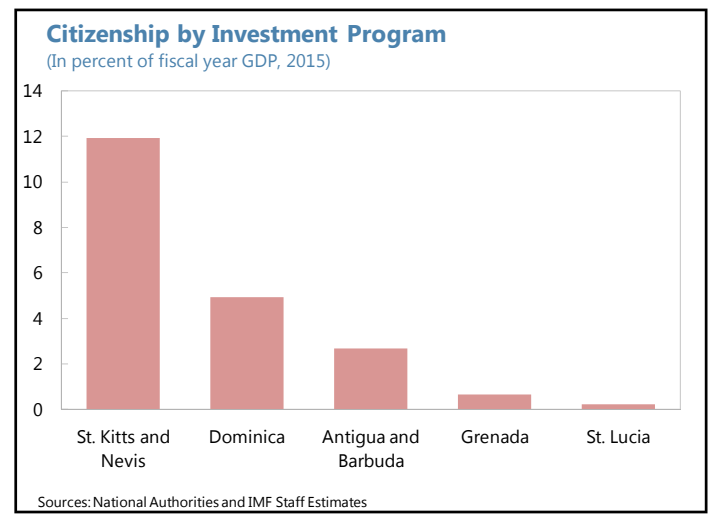
Rasmussen, 2004; Noy, 2009; Cavallo and Noy, 2011; Cavallo, Galiani, Noy and Pantano, 2013; and $\mathrm{Xin} \mathrm{Xu}$, El-Ashram and Gold, 2015). A challenge however, is that CIP revenues are difficult to predict and may be subject to a sudden stop given the increasing scrutiny from advanced economies and growing competition, especially within the ECCU. Also, there is a possible negative externality that affects the stability of the CBI revenues if there are reputational spillovers to the CBI programs of other countries in the region. ${ }^{3}$

\footnotetext{
1 Prepared by Alejandro Guerson.

${ }^{2}$ In the case of St. Vincent and the Grenadines, where a CBI does not exist, setting up a SF for ND may require debt issuance.

${ }^{3}$ This is because the benefits of a program are fully internalized by the country issuing a passport but the potential costs in the case of the granting of a passport to problematic beneficiaries could affect the reputation of the CBI programs of the region as a whole, therefore undermining the prospective revenues of other countries. This situation can distort the incentives towards reducing the efforts on due-diligence checks and therefore exacerbates the risks of revenue erosion or outright loss.
} 


\section{An SF for ND could also help address debt sustainability challenges affecting all ECCU} countries. Public debt is high in all ECCU countries, imposing a constraint on the ability to borrow in the face of ND. Acevedo (2014) finds that tropical storms and hurricanes have a negative effect on growth, as well as transitory and permanent effects on debt accumulation for a subsample of Caribbean countries. This fact supports the case for the use of CBI financing for the start-up and the subsequent funding of the SFs. The saved CBI flows would be allocated for reconstruction after NDs, effectively reducing the need to issue additional debt in the face of an ND shock. Restricting the use of CIP flows in this manner would also reduce the scope for using this unreliable source of revenue to increase recurrent expenditures, further reinforcing fiscal sustainability.

3. The annex is organized in four sections. Section B presents some reasons why government of countries affected by large and recurrent ND should consider self-insurance to cope with ND, commensurate to their frequency, intensity, and anticipated fiscal costs. Section C presents the methodology used in the simulation exercise. Section D presents the calibration of the model parameters for each ECCU country. Section E presents the results.

\section{B. Why a SF for self-insurance against ND?}

4. Existing options to insure against ND are insufficient and costly. The most vulnerable segments of the population are generally uninsured or under-insured and often the most exposed. ${ }^{4}$ This means that governments typically need to cover a significant share of private losses and to provide social support, in addition to the costs of rebuilding destroyed public infrastructure. All ECCU members have access to the Caribbean Catastrophic Risk Insurance Fund (CCRIF), but the costs are high and the coverage purchased is typically limited. General equilibrium calibration analysis indicates that ensuring against ND by issuing catastrophe (CAT) bonds would be beneficial only if the cost of issuing these bonds was significantly smaller than in the data (Borensztein, Cavallo and Jeanne, 2015)..$^{5}$ Moreover, CAT bonds' triggers for payment are imperfectly correlated with the actual losses. ${ }^{6}$

\section{SFs could provide public self-insurance for immediate expenditure needs,} rehabilitation and reconstruction, while supporting fiscal sustainability. In principle, if access to financing were immediately available, an SF might not be necessary. A government could allocate the fiscal savings to debt reduction (of an amount commensurate to the expected cost of

\footnotetext{
${ }^{4}$ For example, low income households often settle in lands that are more exposed to flooding, and their income is also usually more affected by NDs (for example, as it affects farming and transport).

${ }^{5}$ CAT bonds are inherently risky, typically pay coupons of Libor plus a spread in the range of 3-20 percent, and have maturities of less than 3 years. See also Froot, 2001; Cummins, 2008 and 2012; and Cummins and Mahul, 2009.

${ }^{6}$ CAT bonds are structured in four types of triggers for payment: (i) indemnity (trigger by the actual losses in excess of a specific threshold0; (ii) modeled loss (based on catastrophe modeling run with the event parameters to measure if the modeled losses are above a specified threshold); (iii) indexed to industry loss (triggered when the insurance industry loss reached a specified threshold, as determined by a specified agency); (iv) parametric (trigger is indexed to the natural hazard caused by nature, such as wind speed in a specific location for a hurricane); and (v) parametric index (models used to compute an approximated loss, de-facto it is a hybrid parametric/modeled loss).
} 
reconstruction) and save on interest expenditures, and then borrow when hit by a ND to cover the costs. However, there are several reasons why this strategy is difficult to implement in practice. First, access to financing is typically not sufficiently rapid, especially for small countries like those in the ECCU which do not have access to international financial markets. Increasing official loans and changing the scope of existing official loans (i.e. to ND relief and rehabilitation) would typically involve a lengthy process. Furthermore, the disbursement of grants from bilateral donor countries also requires lengthy application and approval processes, and time to materialize. Access to rapid domestic financing could also be limited, especially if the ND shock affects financial institutions' asset quality, and if deposits decline as the population copes with the shock. Fiscal savings for reconstruction in a dedicated SF would facilitate long-term fiscal sustainability by imposing a recurrent saving discipline if specified in an amount that is commensurate to the expected reconstruction costs.

\section{Some countries in the region already have SFs, but none is specifically targeting the} financing of ND fiscal costs. The Sugar Industry Diversification Fund in St. Kitts and Nevis is a national development fund that is also financed with CBI inflows, and it is set up as a public fund. It was established in 2006 with the objective of supporting the financing of economic diversification from the sugar industry through training and research. In 2011, its focus was expanded to maintain stability and the financing of industries. It provides budgetary support, undertakes direct social spending, and supports subsidized credit by banks. In 2014, Grenada launched a National Transformation Fund, funded with CBI revenues. This fund makes transfers to the budget for the repayment of arrears and for investment projects.

\section{Methodology}

\section{The starting point is to estimate an empirical model for each economy that captures} the effects of ND on output and government finances. To this end, a Vector Auto-regression Model (VAR) is estimated for each country. The endogenous variables in the VAR estimates include the cyclical components of GDP; government revenues excluding grants; grants; current primary expenditures; and capital expenditures. ${ }^{7}$ ND shocks are identified by including control variables that account for other major sources of shocks. The vector of control variables includes the U.S. real effective exchange (to capture competitiveness pressures given that the EC dollar is pegged to the U.S. dollar); the oil price (all countries are highly dependent on oil imports); the cyclical component of the U.S. output (the main source of tourist revenues); and a dummy for the September 2001 shock that significantly disrupted tourism exports. This results in estimated vectors of residuals in each country's VAR that are orthogonal to the non-ND shocks captured by the controls. The underlying assumption is that the control variables "remove" the main alternative sources of fiscal shocks from the estimated vector residuals, resulting in a streamlined estimated distribution of

\footnotetext{
${ }^{7}$ The cyclical components used in the empirical model are calculated as the ratio of the variable with respect to its estimated trend. The cyclical components of GDP are estimated using the Hodrick-Prescott filter on 1990-2015 annual data. All variables expressed in real terms using the GDP deflator. The identification of shocks is performed according to the Choleski decomposition.
}

(continued) 
shocks that includes ND as the most significant shock remaining. The estimated vectors of residuals therefore include ND and other smaller shocks. ${ }^{8}$

\section{The second step is to run a Monte-Carlo experiment, which involves generating a large} number of simulations for the period 2016-2030 with the estimated models. Each simulation is a projection consisting of a sequence of the five endogenous variables in the models. 1000 simulations are run for each country, each affected by a sequence of simulated random shocks, which are identified as explained above. The random shocks are drawn from the probability density functions estimated from the models' residuals, which are assumed to be normally-distributed. Calculated in this way, the simulations generate data series that mimic historical patterns in terms of volatility, persistence, and co-movement of the five endogenous series in each simulation in response to the shocks. The results are then used to compute probability density functions for each of the five endogenous variables for each year projected. The projections are then expressed as a percent of GDP using a deterministic trend for each endogenous series, and assuming that all trends grow at the same rate as GDP to ensure that ratios as a percent of GDP are stable. ${ }^{9}$ The calculation of the overall balance and the stock of public debt require also a projection of interest expenditures. To this end, the debt stock at the end of the previous year is multiplied by an implicit interest rate path (the ratio of interest expenditures to public debt stock), which is a parameter for calibration. The calculation of interest expenditures is then added to revenues and primary expenditures to compute the public debt stock dynamics using the debt accumulation identity, which is expanded to also include the budget financing flows vis-à-vis the SF.

\section{The third step is to identify the occurrence of natural disasters in each simulation, as} needed to inform the triggering of financing flows vis-à-vis the SFs. To this end, the simulations include an algorithm that identifies a ND as the largest $X$ percent fiscal deteriorations. The fiscal deteriorations are computed as the sum of the year-on-year changes of (i) non-grant revenue (with a negative sign as tax revenues would tend to decline along with output during ND); (ii) grant revenues (which would presumably increase after ND as donor partners increase their supports) (iii) current primary expenditure (as more social assistance and goods and services are needed); and (iv) capital expenditure (on account of additional expenditures for rehabilitation and reconstruction). The algorithm then looks at the distribution of this sum, and identifies as a ND all the random realizations that fall in the highest $X$ percent tail of the of the probability density function of this sum. In this way, if (statistically) in a given simulation non-grant revenues decline significantly, and grant revenues, current primary expenditures, and capital expenditures increase significantly (a typical pattern after a ND), then that random simulation is identified as a ND. Notice the calibration of the probability threshold is important as it determines the annual frequency of NDs in the

\footnotetext{
8 The sample data used in the estimation spans 1990-2015.

${ }^{9}$ It is therefore implicitly assumed that the deflators of GDP and the remaining fiscal variables change at the same rate in the projections. The starting point of the projections is the end point of the estimated trend in the sample period.
} 
simulations. For example, if recent episodes indicate that a ND occurs every 5 years, then the probability of a ND should be set at 0.2 .

\section{The fourth step is to specify the SFs financing flows vis-à-vis the budget. The} simulations assume that in years with no ND, as identified by the algorithms explained above, the budget generates an additional overall balance surplus that is deposited in the SF. ${ }^{10}$ These budget contributions to the SF are modeled as a fixed parameter as a percent of the previous year GDP. The amount of this annual saving is calibrated to achieve the financial sustainability of the Fund with a sufficiently low probability of depletion, thus ensuring that the SF stock of assets is stable in expected terms ${ }^{11}$. In the event a ND occurs, a financing inflow to the budget from the SF takes place. This budget financing is computed as the sum of four components:

+ Gap of non-grant revenues below trend. Captures the decline in tax and non-tax revenues that typically take place after natural disasters as a result of a decline in economic activity and tax compliance.

- Gap of grant revenues above trend. Grants tend to be higher after natural disasters as a result of an increase in donor support, reducing the need for financing flows from the Fund.

+ Gap of current primary expenditure above trend. Captures higher expenditures in social support and rehabilitation of infrastructure after natural disasters. An additional fixed amount as a percent of GDP is added that captures below-trend reprioritization of spending.

+ Gap of capital expenditure above trend. Captures the higher public investment that typically follows NDs. An additional fixed amount as a percent of GDP is added that captures below-trend reprioritization of spending.

The contributions to the budget continue until the year in which each indicator returns to a level that is below the value in the year prior to the natural disaster.

\section{The modeling strategy also accounts for expenditure re-prioritization, resulting in a} realistic assessment of the size of SFs. In practice, a significant share of the fiscal space for social support and reconstruction after ND is obtained by way of reallocation and re-prioritization: some pre-ND

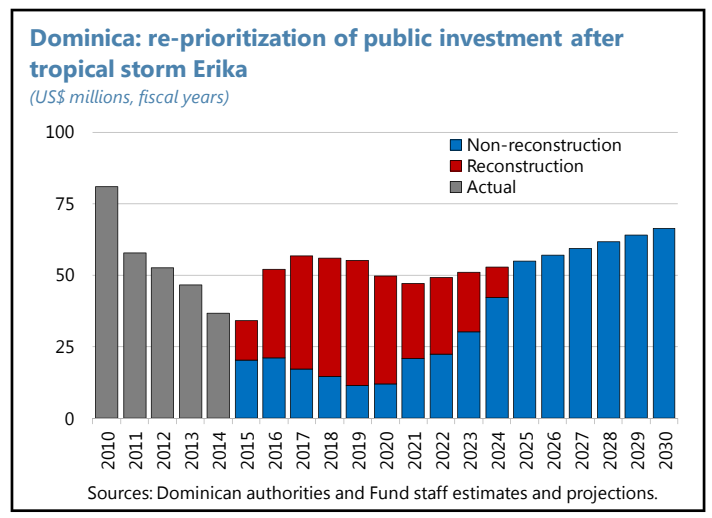

\footnotetext{
${ }^{10}$ If the simulations result in a fiscal deficit, then there would be a need to issue public debt to finance the required contribution to the Fund.

${ }^{11}$ In other words, as needed to ensure that the saving rate is of amount commensurate with the fiscal costs of ND. if saving inflows into the SF are set too high (low), then the size of the Fund would tend to increase (decrease) in expected terms, and would therefore be financially unsustainable (accumulate unnecessary assets).
} 
allocations are postponed or cancelled. The text chart illustrates the case of re-prioritization in the case of Dominica, after it was affected by Tropical storm Erika in August 2015. As a result, the reconstruction expenditures do not require an equivalent increase in public investment. This is the reason for the additional savings explained above relative to the estimated trends allowed for the current primary and capital expenditures.

12. The modeling of the SF also includes an assumption for the initial stock value, the start-up cost. This initial amount of assets affects the probability of depletion over a time horizon. As the proposal assumes that the start-up cost of establishing a SF is funded with existing CBI assets, it has not been added to the debt stock at the beginning of the projection horizon (end2015).

13. The simulations are then used to compute probabilistic public debt projections, taking into account the government budget financing flows vis-à-vis the SF. The simulated series of revenues and primary expenditures allow the calculation of primary balances and public debt dynamics using the debt accumulation identity. In years with no ND, the budget contributes the specified savings to the SF -as opposed to reducing debt in that amount. If a ND occurs, the SF is used to finance the additional fiscal needs as specified in the SF disbursement rules above -as opposed to issuing public debt. ${ }^{12}$

\section{Calibration}

14. The macroeconomic parameters are calibrated consistent with staff's macroeconomic frameworks for each ECCU country. These include potential GDP growth rate (which is also applied to the trend growth of the remaining simulated endogenous fiscal indicators in the simulations to obtain stable shares as a percent of GDP in the long-term); the implicit interest rate (interest payments / debt stock; fiscal consolidation targets in percent of GDP (allocated across the four simulated endogenous fiscal variables in line with the macroeconomic frameworks in the WEO). Appendix 1 shows the specific parametric calibrations used in the simulations for each country.

\section{The parameters affecting the SF are calibrated to achieve its long-term financial} sustainability with a low probability of depletion. A key parameter is the ND probability threshold. This parameter was set consistent with the historical frequency of ND. The initial size of the Fund stock is set to obtain a probability of depletion within the next ten years that is at most of 10 percent, so that the SF is of size enough to cover most of the funding, except in the most extreme cases. The budget saving flows into the SF in years with no ND are set last, consistent with ensuring that the SF stock of assets does not increase or decrease in expected terms.

\section{The remaining parameters for calibration specify the amount of SF financing to the} budget after a ND, including for spending re-prioritization. To this end, "base" levels of capital expenditures and current primary expenditures are calibrated, with "base" defined as the level of

\footnotetext{
12 If the SF is depleted at some point in a simulation, it is assumed that the financing that the SF cannot provide is covered with debt issuance.
} 
spending that would prevail in a year in which there is no spending associated with the occurrence of a ND. As explained above, the SF is assumed to disburse financing to the budget after a ND of an amount equivalent to the gap between the simulated amounts of non-grant revenues, current primary expenditures, and capital expenditures and their trends, respectively (net of the simulated increase in grants above trend). As the estimated trends in the sample period can be assessed to be higher or smaller that the estimated trend, an additional parameter is introduced for capital and current primary expenditures that allows specifying an additional gap above-and-beyond the gap with respect to the estimated trend. These financing flows to the budget continue during the years after a ND for as long as the simulated level of spending is higher than the level registered before the ND. In other words, the SF finances the spending "hump" above the calibrated "base" levels.

\section{E. Results}

\section{Under the parameter calibrations proposed, the SF of all countries would be financially} sustainable with a low probability of depletion. Countries would need SFs stocks in the range of 6-12 percent of GDP to self insure against ND, and annual savings in years with no ND of 0.4-1.9 percent of GDP to achieve the SF's financial sustainability with a low probability of depletion (text table). For each ECCU country, Figure 1 shows the sensitivity of the size of a SF to changes in the calibrated frequency of natural disasters, as determined by the probability threshold. For example, if the probability of a ND were instead set one notch higher than in the specific calibration used in the simulations, the annual budget savings deposited in the SF would need to be higher by around 0.2 percent of GDP (Grenada; St. Lucia; St. Vincent and the Grenadines) or around 0.5 percent of GDP (Antigua and Barbuda; Dominica; St. Kitts and Nevis). Figure 2 shows the probability of depletion of the SF when the ND probability is calibrated at the value used in the proposal, and shows the probability of depletion of the SF for different initial sizes of SF stock of assets within a ten-year horizon. According to Figure 2, lowering the proposed stock of assets in the SF by 2 percent of GDP would result in a probability of depletion of about 10 percent or higher.

\begin{tabular}{|c|c|c|c|c|c|c|}
\hline \multicolumn{7}{|c|}{$\begin{array}{l}\text { Government Saving Funds for Natur } \\
\text { (In percent of GDP unless otherwise indicated) }\end{array}$} \\
\hline & ATG & DMA & GRD & KNA & LCA & VCT \\
\hline Fund size & 12.00 & 10.00 & 6.00 & 10.00 & 8.00 & 8.00 \\
\hline Annual budget saving if no ND & 1.90 & 1.50 & 0.35 & 1.90 & 1.05 & 0.95 \\
\hline Probability of fund depletion, units & 0.05 & 0.08 & 0.04 & 0.07 & 0.06 & 0.03 \\
\hline Annual expected use of the fund if ND & 8.7 & 5.9 & 3.0 & 4.8 & 3.4 & 2.6 \\
\hline Average fiscal cost of simulated ND 1/ & 57.9 & 29.3 & 29.7 & 19.3 & 13.7 & 12.9 \\
\hline \multicolumn{7}{|c|}{$\begin{array}{l}\text { Source: Staff calculations based on authorities' data. } \\
1 \text { / Includes the estimated average decline in revenues and increase in primary expenditures in the simulations, } \\
\text { net of the expected increase in donor grants. }\end{array}$} \\
\hline
\end{tabular}


18. Under these calibrations, public debts in general decline towards the regional target of 60 percent of GDP by 2030 in expected terms, but in some cases with significant uncertainty. This is obtained after accounting for the financing flows between the government budget and the SF depending on the occurrence of the ND in the simulations. Figure 3 shows that public debt would decline to around 60 percent of GDP or lower by 2030 in expected terms in all countries, in line with regional commitments. However, the results indicate that there is a significant probability that the target is missed if ND hit ECCU countries more frequently and/or harder than expected. This is particularly the case in Dominica and St. Kitts and Nevis.

\section{Supporting the SF with a strong institutional setup is critical to avoid political} pressures for spending or opportunistic appropriations. A strong institutional design should include unambiguous budget contribution and disbursement rules, with triggers based on verifiable criteria, a clearly-stated objective, and strict information disclosure requirements to ensure the transparency of its operations.

\section{References}

Acevedo, S. 2014. "Debt, Growth and Natural Disasters: A Caribbean Trilogy" IMF Working Paper $14 / 125$.

Barro, R. 2006. "Rare Disasters and Asset Markets in the Twentieth Century." Quarterly Journal of Economics, 121: 823-899.

Barro, R. 2009. "Rare Disasters, Asset Prices and Welfare Costs." American Economic Review, 99(1): 243-264.

Cavallo, E. A., and Noy, I., 2011. "Natural Disasters and the Economy: A Survey." International Review of Environmental and Resource Economics, 5: 63102.

Cavallo, E., Galiani,S., Niy, I., and Pantano, J., 2013. "Catastrophic Natural Disasters and Economic Growth." Review of Economics and Statistics, 95(5): 1549\{1561.

Cummins, J.D. 2008. \CAT Bonds and Other Risk-Linked Securities: State of the Market and Recent developments." Risk Management and Insurance Review, 11(1): 23-47.

Cummins, J.D. 2012. "CAT Bonds and Other Risk-Linked Securities: Product Design and Evolution of the Market." In Extreme Events and Insurance: 2011 Annus Horribilis, ed. Christophe Courbage and Walter R. Stahel, 39-61. The Geneva Association.

Cummins, J.D., and O. Mahul. 2009. Catastrophe risk financing in developing countries: principles for public intervention. World Bank Publications. 
El-Ashram, A., Gold, J. Xu, X., 2015. "Too Much of a Good Thing? Prudent Management of Inflows under Economic Citizenship Programs." IMF Working Paper 15/93.

Froot, Kenneth A. 2001. "The Market for Catastrophe Risk: a Clinical Examination."

Journal of Financial Economics, 60(2): 529-571.

Lee, Jin-Ping, and Min-Teh Yu. 2007. "Valuation of Catastrophe Reinsurance with Catastrophe Bonds." Insurance: Mathematics and Economics, 41(2): $264\{278$.

Noy, Ilan. 2009. "The Macroeconomic Consequences of Disasters." Journal of Development Economics, 88(2): 221-231.

Rasmussen, T. N. 2004. "Macroeconomic Implications of Natural Disasters in the Caribbean." IMF Working Paper 04/224. 
Figure 1: Annual budget savings required for the financial sustainability of the SFs

Antigua and Barbuda

Annual budget contributions to the Fund for natural disasters (ND) (percent of GDP)

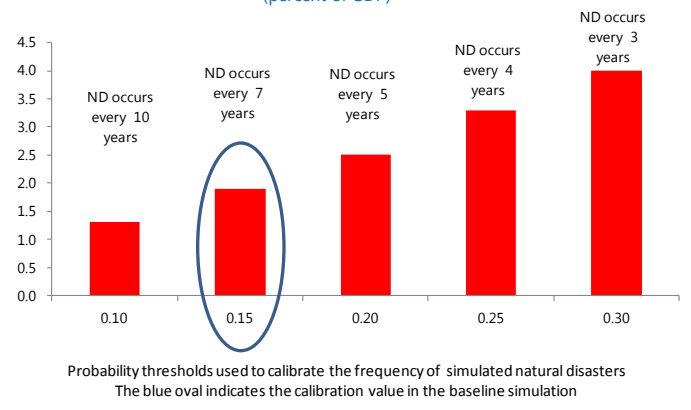

Grenada disasters (ND)

(percent of GDP) ND

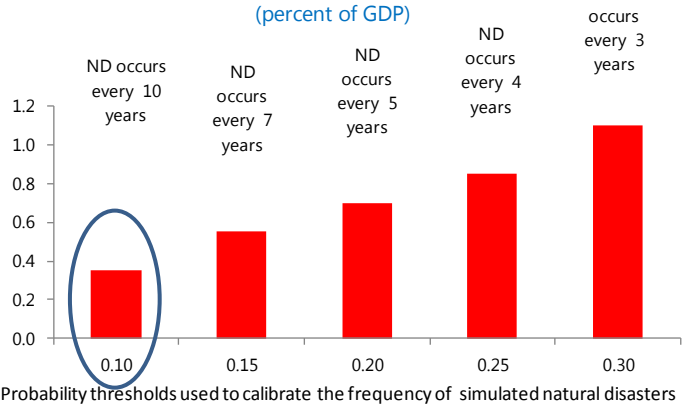

Probability thresholds used to calibrate the frequency of simulated natural disasters The blue oval indicates the calibration value in the baseline simulation

\section{St. Lucia}

Annual budget contributions to the Fund for natural disasters (ND) (percent of GDP)

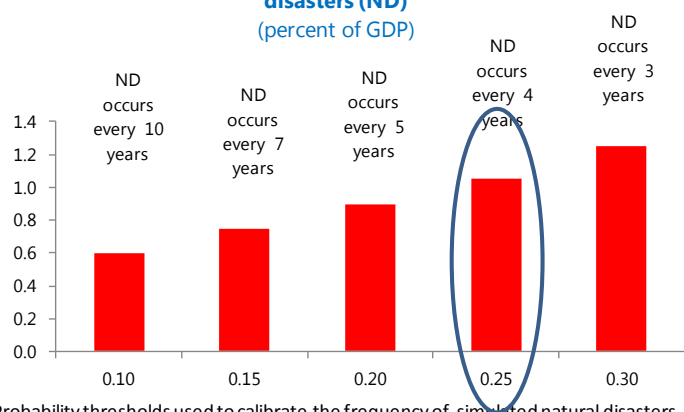

Probability thresholds used to calibrate the frequency of sim arted natural disasters The blue oval indicates the calibration value in the baseline simulation
Dominica

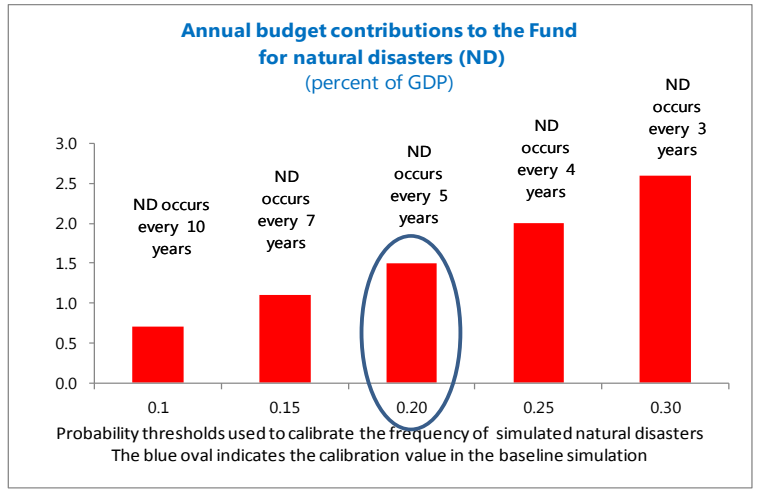

\section{St. Kitts and Nevis}

Annual budget contributions to the Fund for natural disasters (ND) ND

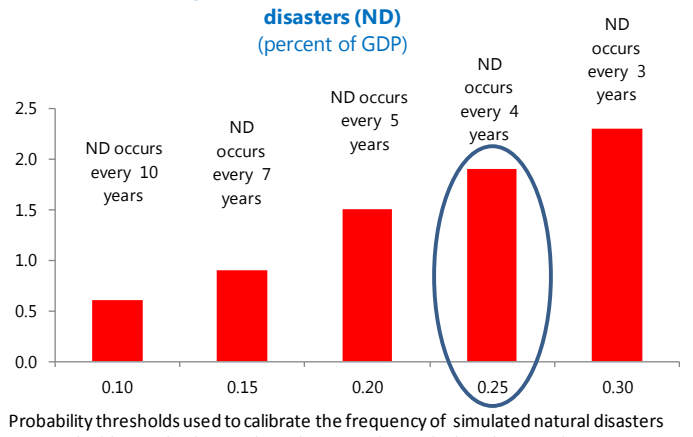

Probability thresholds used to calibrate the frequency of simulated natural disaster The blue oval indicates the caibration value in the baseline simulation

\section{St. Vincent and the Grenadines}

Annual budget contributions to the Fund for natural disasters (ND)

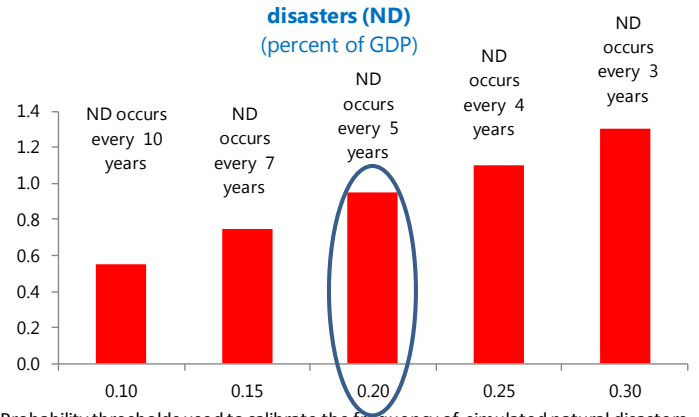

Probability thresholds used to calibrate the requency of simulated natural disasters The blue oval indicates the calibration value used in the baseline simulation 


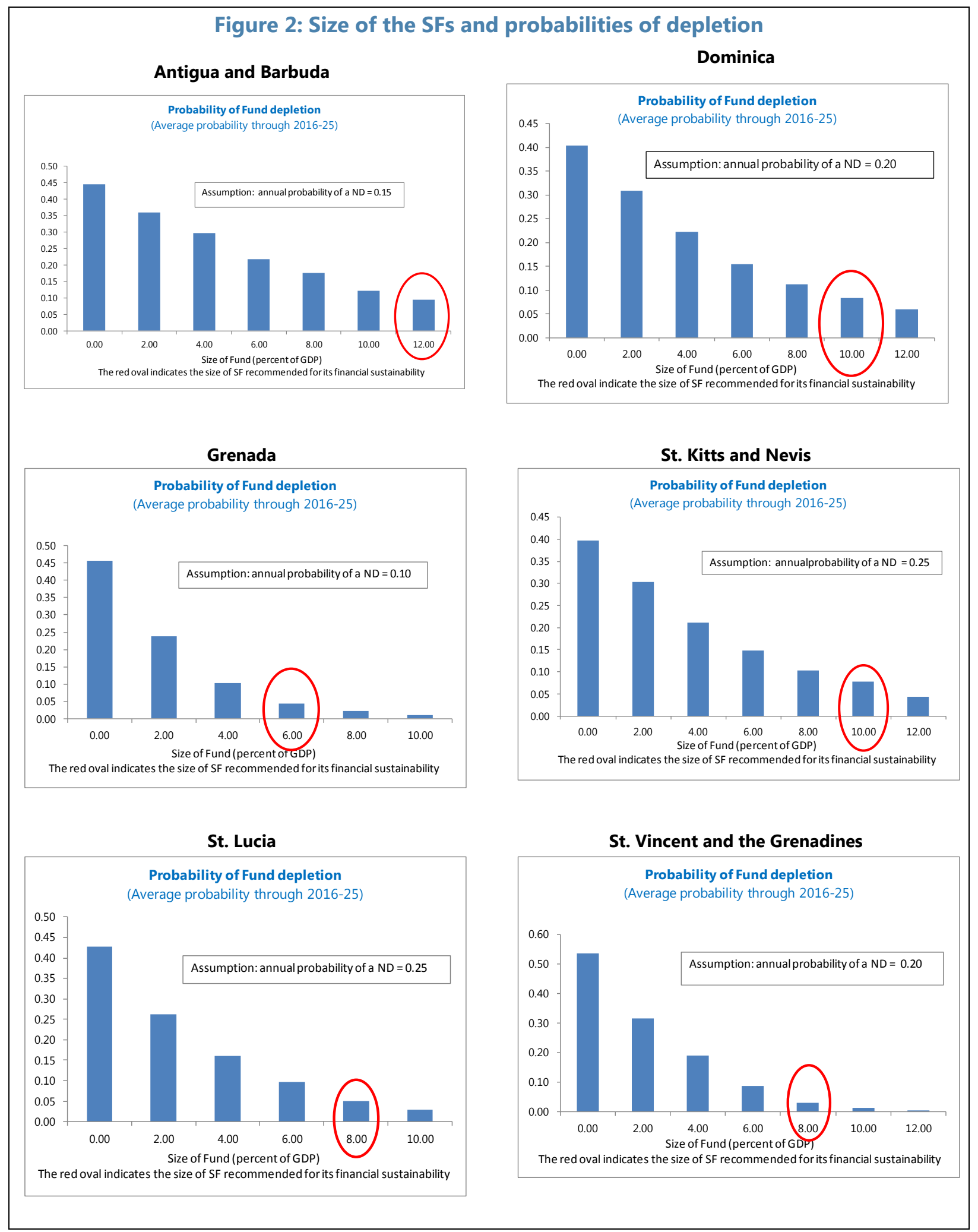


Figure 3: Public debt fan charts including budget financing flows vis-à-vis the SFs

Antigua and Barbuda

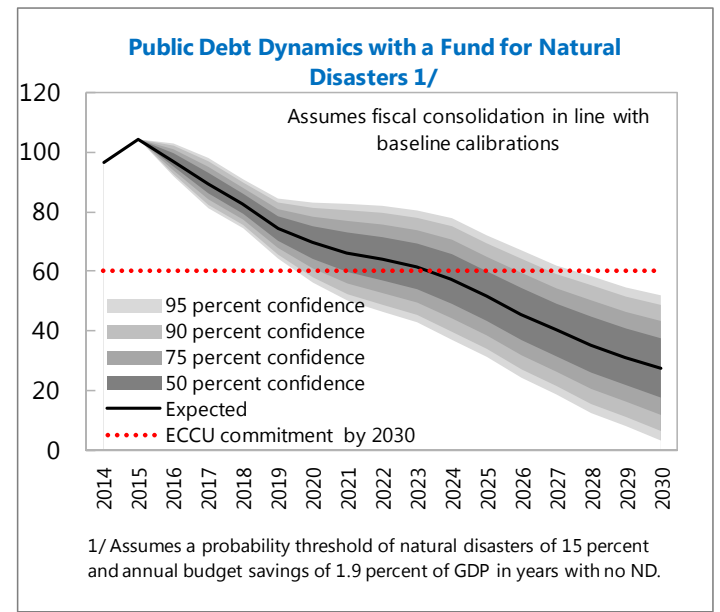

\section{Grenada}

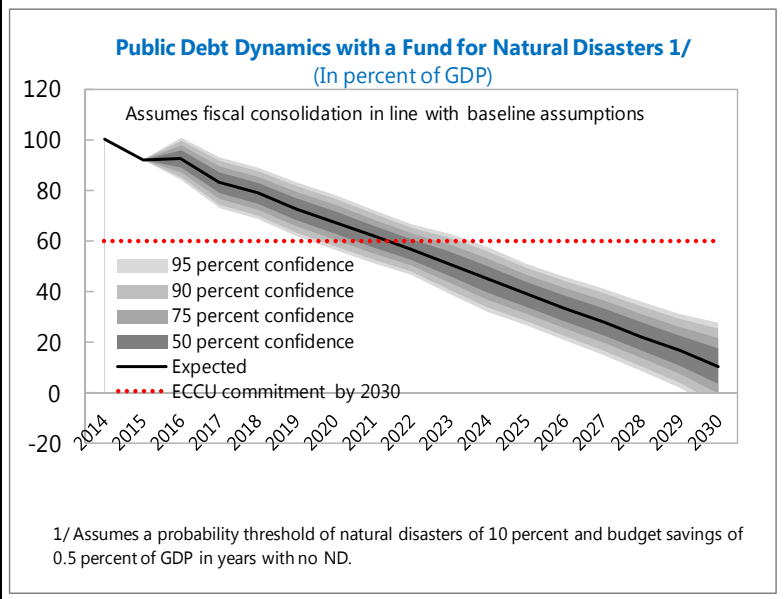

\section{St. Lucia}

Public Debt Dynamics with a Fund for Natural Disasters 1/ (In percent of GDP)

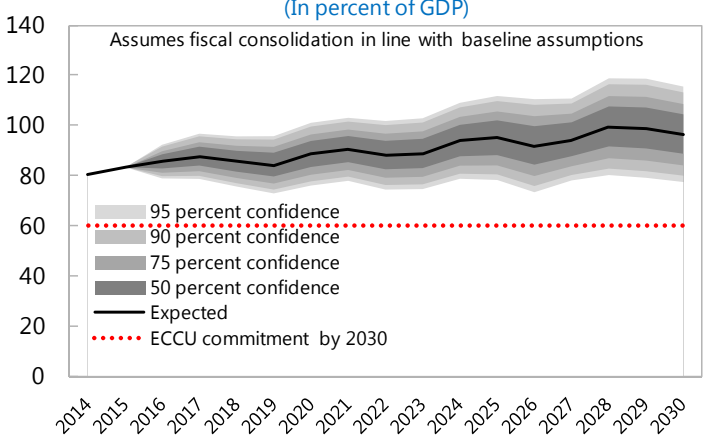

1 / Assumes a probability threshold of natural disasters of 25 percent and annual budget savings of 1.05 percent of GDP in years with no ND.

\section{Dominica}

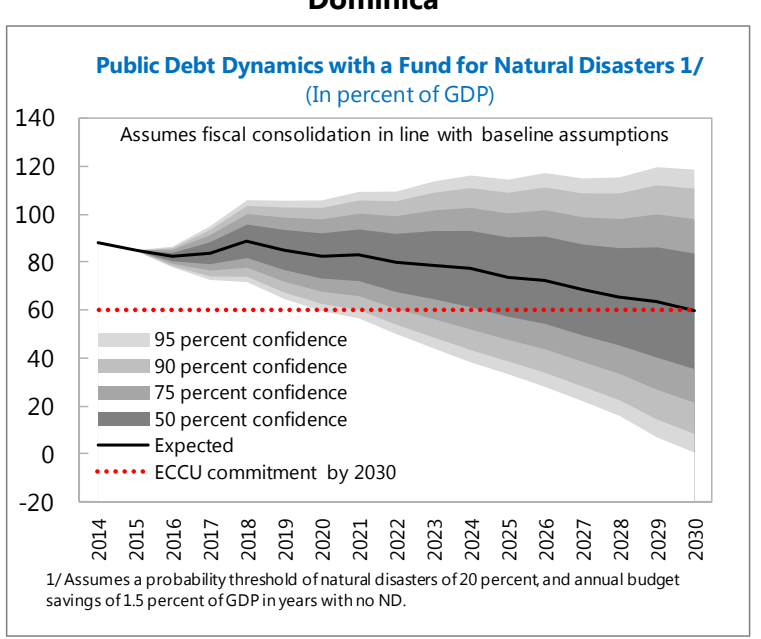

\section{St. Kitts and Nevis}

Public Debt Dynamics with a Fund for Natural Disasters $1 /$ (In percent of GDP)

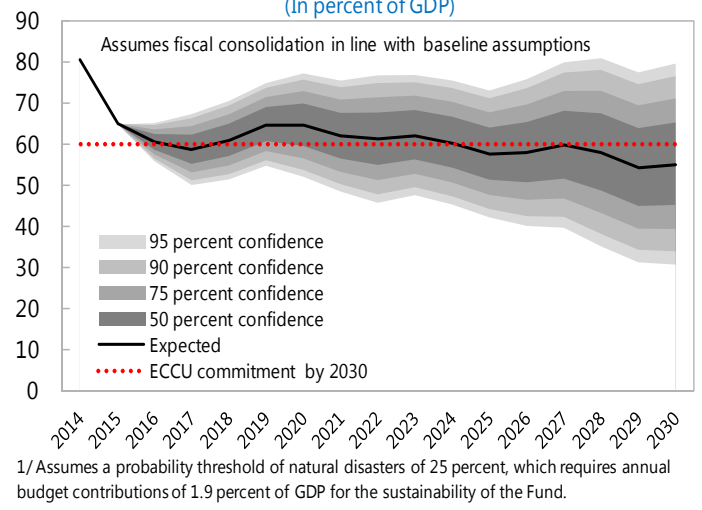

\section{St. Vincent and the Grenadines}

Public Debt Dynamics with a Fund for Natural Disasters 1/ (In percent of GDP)

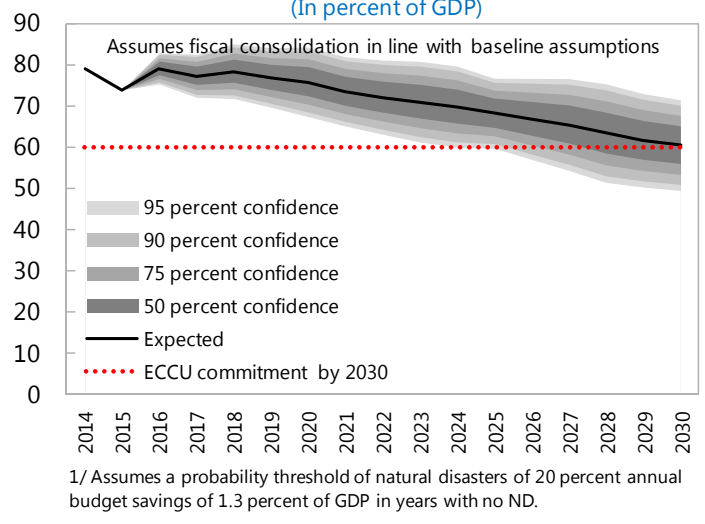


Appendix 1: Parameter Calibrations

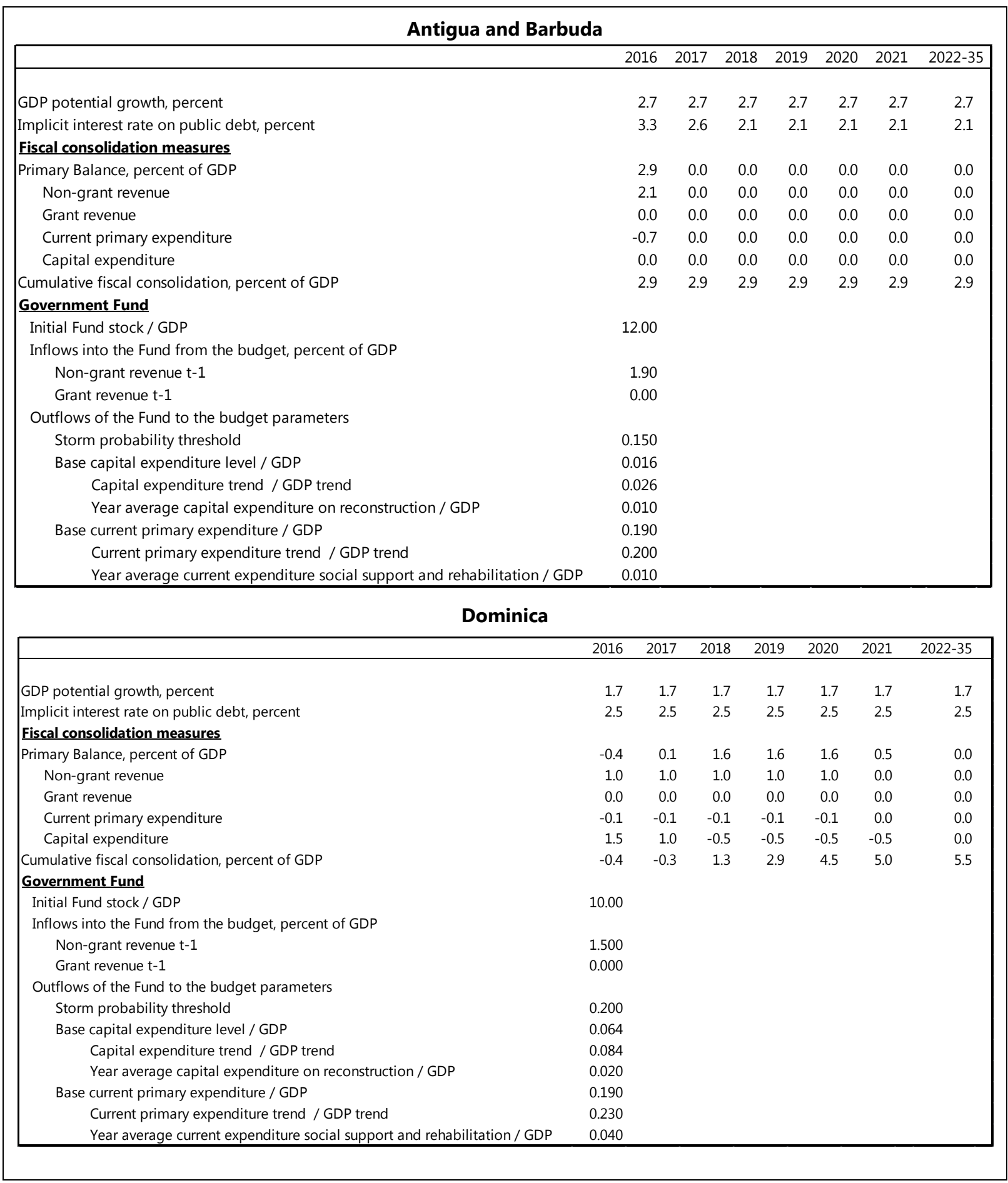




\section{Grenada}

\begin{tabular}{|c|c|c|c|c|c|c|c|}
\hline & 2016 & 2017 & 2018 & 2019 & 2020 & 2021 & $2022-35$ \\
\hline GDP potential growth, percent & 2.7 & 2.7 & 2.7 & 2.7 & 2.7 & 2.7 & 2.7 \\
\hline Implicit interest rate on public debt, percent & 3.6 & 3.5 & 3.4 & 3.3 & 3.3 & 3.3 & 3.3 \\
\hline \multicolumn{8}{|l|}{ Fiscal consolidation measures } \\
\hline Primary Balance, percent of GDP & 0.8 & 0.7 & 0.2 & 0.1 & 0.0 & 0.0 & 0.0 \\
\hline Non-grant revenue & 0.0 & 0.0 & 0.0 & 0.0 & 0.0 & 0.0 & 0.0 \\
\hline Grant revenue & 0.0 & 0.0 & 0.0 & 0.0 & 0.0 & 0.0 & 0.0 \\
\hline Current primary expenditure & 0.0 & 0.0 & 0.0 & 0.0 & 0.0 & 0.0 & 0.0 \\
\hline Capital expenditure & -0.8 & -0.7 & -0.2 & -0.1 & 0.0 & 0.0 & 0.0 \\
\hline Cumulative fiscal consolidation, percent of GDP & 0.8 & 1.5 & 1.7 & 1.8 & 1.8 & 1.8 & 1.8 \\
\hline \multicolumn{8}{|l|}{ Government Fund } \\
\hline Initial Fund stock / GDP & 6.0 & & & & & & \\
\hline \multicolumn{8}{|l|}{ Inflows into the Fund from the budget, percent of GDP } \\
\hline Non-grant revenue $\mathrm{t}-1$ & 0.4 & & & & & & \\
\hline Grant revenue t-1 & 0.0 & & & & & & \\
\hline \multicolumn{8}{|l|}{ Outflows of the Fund to the budget parameters } \\
\hline Storm probability threshold & 0.1 & & & & & & \\
\hline Base capital expenditure level / GDP & 0.1 & & & & & & \\
\hline Capital expenditure trend / GDP trend & 0.1 & & & & & & \\
\hline Year average capital expenditure on reconstruction / GDP & 0.0 & & & & & & \\
\hline Base current primary expenditure / GDP & 0.2 & & & & & & \\
\hline Current primary expenditure trend / GDP trend & 0.2 & & & & & & \\
\hline Year average current expenditure social support and rehabilitation / GDP & 0.0 & & & & & & \\
\hline
\end{tabular}

\section{St. Kitts and Nevis}

\begin{tabular}{|c|c|c|c|c|c|c|c|}
\hline & 2016 & 2017 & 2018 & 2019 & 2020 & 2021 & $2022-35$ \\
\hline GDP potential growth, percent & 2.5 & 2.5 & 2.5 & 2.5 & 2.5 & 2.5 & 2.5 \\
\hline Implicit interest rate on public debt, percent & 3.5 & 3.5 & 3.5 & 3.5 & 3.5 & 3.5 & 3.5 \\
\hline \multicolumn{8}{|l|}{ Fiscal consolidation measures } \\
\hline Primary Balance, percent of GDP & -4.2 & -0.2 & 1.7 & 0.1 & 1.8 & 1.8 & 0.0 \\
\hline Non-grant revenue & -6.1 & -2.1 & -0.2 & -1.8 & -0.1 & -0.1 & 0.0 \\
\hline Grant revenue & 0.0 & 0.0 & 0.0 & 0.0 & 0.0 & 0.0 & 0.0 \\
\hline Current primary expenditure & -1.9 & -1.9 & -1.9 & -1.9 & -1.9 & -1.9 & 0.0 \\
\hline Capital expenditure & 0.0 & 0.0 & 0.0 & 0.0 & 0.0 & 0.0 & 0.0 \\
\hline Cumulative fiscal consolidation, percent of GDP & -4.2 & -4.4 & -2.7 & -2.6 & -0.8 & 1.1 & 1.1 \\
\hline \multicolumn{8}{|l|}{ Government Fund } \\
\hline Initial Fund stock / GDP & 10.0 & & & & & & \\
\hline \multicolumn{8}{|l|}{ Inflows into the Fund from the budget, percent of GDP } \\
\hline Non-grant revenue $\mathrm{t}-1$ & 1.9 & & & & & & \\
\hline Grant revenue t-1 & 0.0 & & & & & & \\
\hline \multicolumn{8}{|l|}{ Outflows of the Fund to the budget parameters } \\
\hline Storm probability threshold & 0.3 & & & & & & \\
\hline Base capital expenditure level / GDP & 0.0 & & & & & & \\
\hline Capital expenditure trend / GDP trend & 0.1 & & & & & & \\
\hline Year average capital expenditure on reconstruction / GDP & 0.0 & & & & & & \\
\hline Base current primary expenditure / GDP & 0.2 & & & & & & \\
\hline Current primary expenditure trend / GDP trend & 0.2 & & & & & & \\
\hline Year average current expenditure social support and rehabilitation / GDP & 0.0 & & & & & & \\
\hline
\end{tabular}


St. Lucia

\begin{tabular}{|c|c|c|c|c|c|c|c|}
\hline & 2016 & 2017 & 2018 & 2019 & 2020 & 2021 & $2022-35$ \\
\hline GDP potential growth, percent & 2.0 & 2.0 & 2.0 & 2.0 & 2.0 & 2.0 & 2.0 \\
\hline Implicit interest rate on public debt, percent & 2.9 & 2.9 & 2.9 & 2.9 & 2.9 & 2.9 & 2.9 \\
\hline \multicolumn{8}{|l|}{ Fiscal consolidation measures } \\
\hline Primary Balance, percent of GDP & 0.4 & 0.1 & 0.1 & 0.1 & 0.1 & 0.0 & 0.0 \\
\hline Non-grant revenue & 0.3 & 0.0 & 0.0 & 0.0 & 0.0 & 0.0 & 0.0 \\
\hline Grant revenue & 0.0 & 0.0 & 0.0 & 0.0 & 0.0 & 0.0 & 0.0 \\
\hline Current primary expenditure & -0.1 & -0.1 & -0.1 & -0.1 & -0.1 & 0.0 & 0.0 \\
\hline Capital expenditure & 0.0 & 0.0 & 0.0 & 0.0 & 0.0 & 0.0 & 0.0 \\
\hline Cumulative fiscal consolidation, percent of GDP & 0.4 & 0.5 & 0.6 & 0.7 & 0.8 & 0.8 & 0.8 \\
\hline \multicolumn{8}{|l|}{ Government Fund } \\
\hline Initial Fund stock / GDP & 8.0 & & & & & & \\
\hline \multicolumn{8}{|l|}{ Inflows into the Fund from the budget, percent of GDP } \\
\hline Non-grant revenue $\mathrm{t}-1$ & 1.05 & & & & & & \\
\hline Grant revenue $\mathrm{t}-1$ & 0.00 & & & & & & \\
\hline \multicolumn{8}{|l|}{ Outflows of the Fund to the budget parameters } \\
\hline Storm probability threshold & 0.25 & & & & & & \\
\hline Base capital expenditure level / GDP & 0.07 & & & & & & \\
\hline Capital expenditure trend / GDP trend & 0.08 & & & & & & \\
\hline Year average capital expenditure on reconstruction / GDP & 0.01 & & & & & & \\
\hline Base current primary expenditure / GDP & 0.18 & & & & & & \\
\hline Current primary expenditure trend / GDP trend & 0.19 & & & & & & \\
\hline Year average current expenditure social support and rehabilitation / GDF & 0.01 & & & & & & \\
\hline
\end{tabular}

\section{St. Vincent and the Grenadines}

\begin{tabular}{|c|c|c|c|c|c|c|c|}
\hline & 2016 & 2017 & 2018 & 2019 & 2020 & 2021 & $2022-35$ \\
\hline GDP potential growth, percent & 3.0 & 3.0 & 3.0 & 3.0 & 3.0 & 3.0 & 3.0 \\
\hline Implicit interest rate on public debt, percent & 3.3 & 3.3 & 3.3 & 3.3 & 3.3 & 3.3 & 3.3 \\
\hline \multicolumn{8}{|l|}{ Fiscal consolidation measures } \\
\hline Primary Balance, percent of GDP & 0.5 & 0.8 & 0.8 & 0.8 & 0.8 & 0.0 & 0.0 \\
\hline Non-grant revenue & 0.0 & 0.0 & 0.0 & 0.0 & 0.0 & 0.0 & 0.0 \\
\hline Grant revenue & 0.1 & 0.1 & 0.1 & 0.1 & 0.1 & 0.0 & 0.0 \\
\hline Current primary expenditure & 0.0 & 0.0 & 0.0 & 0.0 & 0.0 & 0.0 & 0.0 \\
\hline Capital expenditure & -0.4 & -0.7 & -0.7 & -0.7 & -0.7 & 0.0 & 0.0 \\
\hline Cumulative fiscal consolidation, percent of GDP & 0.5 & 1.3 & 2.1 & 2.9 & 3.7 & 3.7 & 3.7 \\
\hline \multicolumn{8}{|l|}{ Government Fund } \\
\hline Initial Fund stock / GDP & 8.0 & & & & & & \\
\hline \multicolumn{8}{|l|}{ Inflows into the Fund from the budget, percent of GDP } \\
\hline Non-grant revenue $\mathrm{t}-1$ & 1.0 & & & & & & \\
\hline Grant revenue $\mathrm{t}-1$ & 0.0 & & & & & & \\
\hline \multicolumn{8}{|l|}{ Outflows of the Fund to the budget parameters } \\
\hline Storm probability threshold & 0.2 & & & & & & \\
\hline Base capital expenditure level / GDP & 0.0 & & & & & & \\
\hline Capital expenditure trend / GDP trend & 0.0 & & & & & & \\
\hline Year average capital expenditure on reconstruction / GDP & 0.0 & & & & & & \\
\hline Base current primary expenditure / GDP & 0.2 & & & & & & \\
\hline Current primary expenditure trend / GDP trend & 0.2 & & & & & & \\
\hline Year average current expenditure social support and rehabilitation / GDF & 0.0 & & & & & & \\
\hline
\end{tabular}

\section{CInternational Monetary Fund. Not for Redistribution}




\section{Annex IX. Explaining High Unemployment in the ECCU1}

Official unemployment rates in some ECCU countries are very high by international standards. Rates are above 20 percent in Grenada, St. Lucia, and St. Vincent and the Grenadines, even if using a strict definition of unemployment. There are several factors that could explain these high rates such as a rigid wage-setting environment, high reservation wages, the prevalence of natural disasters, and sectoral shifts.

\section{Labor market data in the ECCU, although scarce and from heterogeneous sources,} points to relatively high unemployment levels especially in the years following the global

financial crisis. As seen in Figure 1, in the early 2000s unemployment levels were moderately high in most ECCU countries, while already considerably high in St. Lucia (about 16 percent) and St. Vincent and the Grenadines (about 22 percent). There appeared no clear trend in unemployment up to the years prior to the global financial crisis. However, recent labor force surveys find that unemployment rates have significantly increased in ECCU countries following the global financial crisis. In addition, social security statistics (from NIS) indicate that total employment has remained stagnant since the global crisis, and private sector employment has significantly declined (see Figure 2).
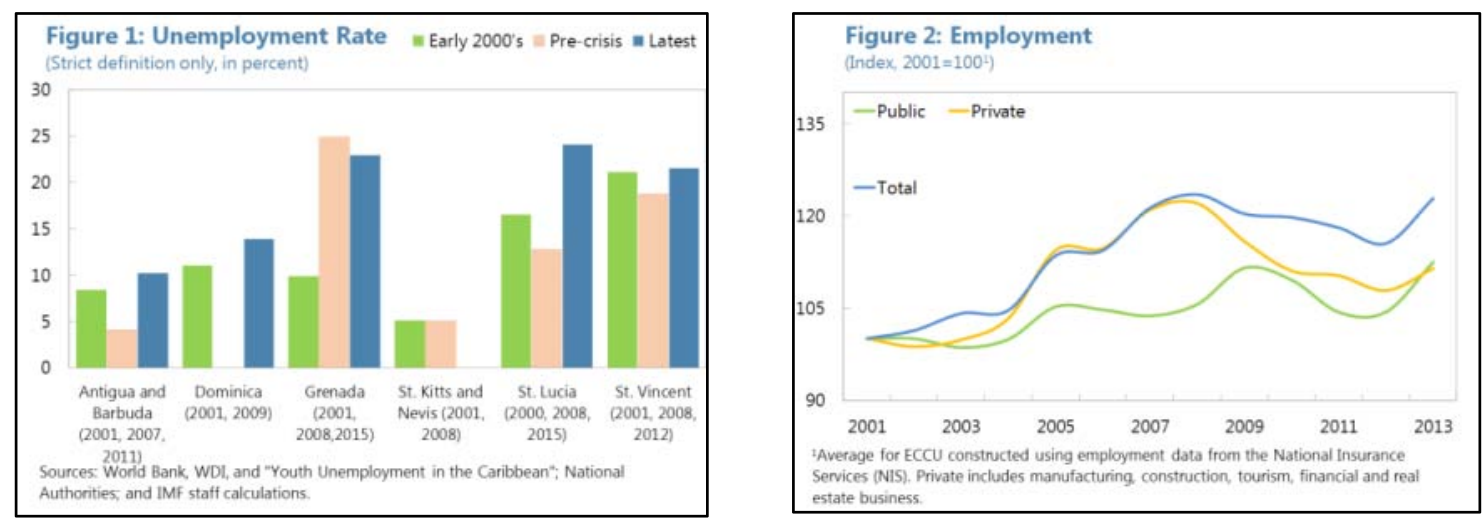

\section{Unemployment rates in Grenada, St. Lucia, and St. Vincent and the Grenadines, are} extremely high and require further analysis to appropriate policy responses. Unemployment is comparatively low in Antigua and Barbuda (10.2 percent) and St. Kitts and Nevis (11 percent), but it is particularly high in Grenada (23 percent), St. Lucia (24 percent), and St. Vincent and the Grenadines (22 percent) ${ }^{2}$. Analyzing the factors behind such high unemployment in ECCU is particularly difficult due to data scarcity and the need to use and compare statistics built through different methodologies (in censuses, labor surveys, and other types of surveys). Fortunately, the two countries with highest unemployment rates (Grenada and St. Lucia) have the most comprehensive

\footnotetext{
${ }^{1}$ Prepared by Ronald James, Jemma Lafeuillee, Mike Li, Gonzalo Salinas (all IMF), and Yevgeniya Savchenko (World Bank), based on preliminary findings from a forthcoming IMF Working Paper.

${ }^{2}$ Unemployment is much higher among the youth as is commonly the case in other countries. In the case of St. Lucia, youth unemployment (ages 15-29) is about 41 percent, and unemployment is much higher for women (27 percent) than for men (20 percent).
} 
labor data among ECCU countries. In particular, St. Lucia, is the only ECCU country that provides consistent time series of labor market statistics since the mid 1990s.

\section{One likely factor behind high unemployment in Grenada and St. Lucia is the recent} disconnect between continued real wage growth and stagnant productivity, especially since the global financial crisis. As seen in Figure 4, average real wages in ECCU countries have been growing over the past decade, especially in the public sector, where the average real wage has increased by 20 percent. This growth contrasts with the virtual stagnation of productivity during this period as moderate growth before the global crisis turned negative afterwards, leading to an increase in unit labor costs. This divergence is actually explained by Grenada and St. Lucia (see Figure 4a), where real wages increased despite a substantial decline in productivity, including in the private sector (see Figure 4b). The growing gap between real wages and productivity since the global crisis could have contributed to the

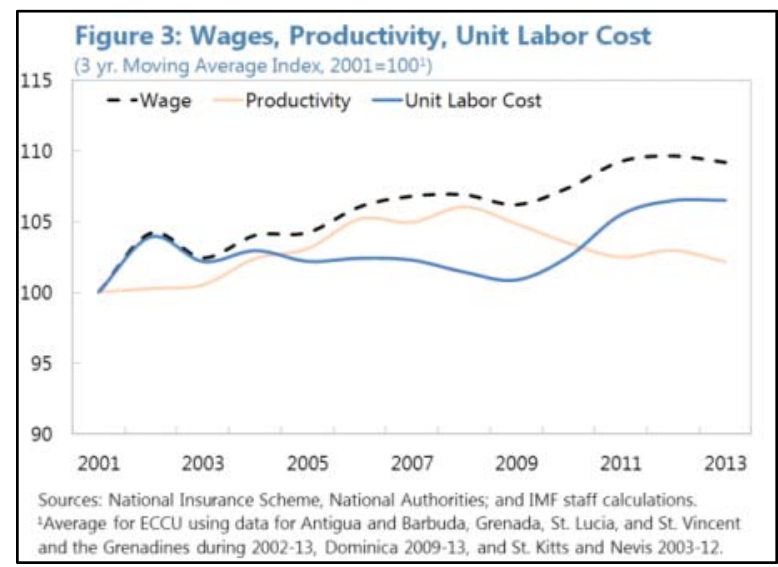
considerable increase in unemployment in St. Lucia, and could have prevented an expected decline in unemployment associated with the economic recovery following Hurricanes in 2004 and 2005 in Grenada.
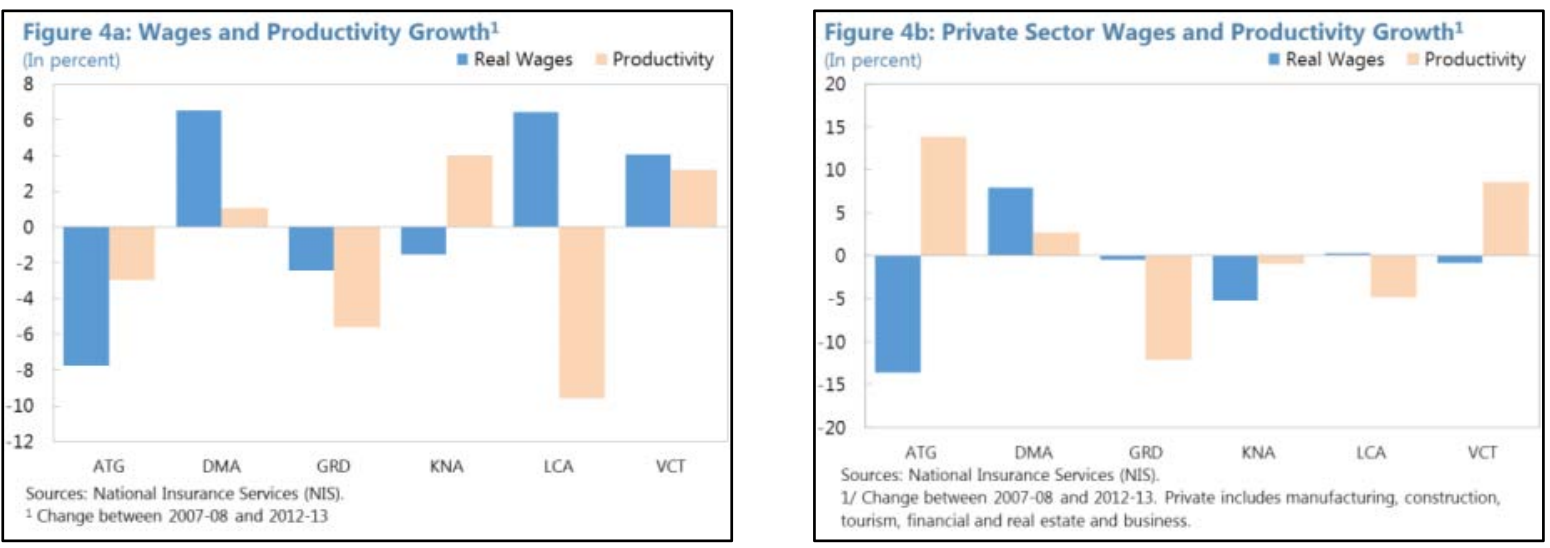

4. A significant factor that could explain the divergence between wages and productivity is strong labor union activity in Grenada and St. Lucia. Wages in Grenada and St. Lucia are generally set through a collective bargaining process and there is strong union activity, especially in the public sector. Unions are much more influential in these countries than in, for example, St. Kitts and Nevis, where unemployment has been much lower since the early 2000s. Strong union activity in the public sector is most important because of the relatively large share of public sector employment (29 percent in St. Lucia and 37 percent in Grenada in 2013) by international standards ${ }^{3}$.

\footnotetext{
${ }^{3}$ Public sector employment estimates based on NIS data and includes Public Administration and Social Security, Education, and Health.
} 
Unions are also active in large private sectors (financial, manufacturing, tourism, and privately run utilities). The wide union coverage in these countries reflects legislation that promotes union membership through direct or indirect requirements (as requisite to access benefits such as medical insurance).

\section{Through demonstration effect, raising public sector wages in both Grenada and St.} Lucia, could have contributed to raising labor costs across sectors. As seen in Figure 5, public and private sector wages increased considerably since 2008 despite the economic slowdown. Growth in the average public sector wage has actually been much faster than in the private sector, particularly in St. Lucia, which could reflect the stronger union activity in the public sector. This could imply that public sector wage growth could have boosted wage growth in the private sector, especially considering that private sector unions often negotiate wages using public sector wages as a benchmark ${ }^{4}$.

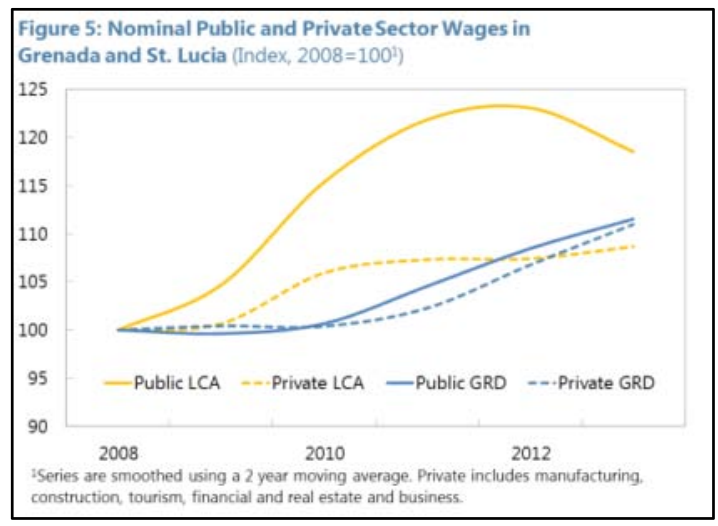

\section{Empirical analysis (VAR regression and Granger test) on Grenada confirms there is a} public wage demonstration effect, and that wage setting seems to be unlinked from productivity or cost of living considerations ${ }^{5}$. The empirical analysis finds bi-directional causality between public and private wages, suggesting both "signaling" and "competition" effects between the two sectors. It also finds that past wages (t-1) in both public and private sectors have a strong predictive power for current wages ( $t$ ), which would be an evidence for nominal wage stickiness. In contrast, CPI and productivity do not seem to affect the nominal wages of either public or private sector, which is in line with the disconnect between real wages and productivity in Grenada seen in Figure 4. This may reflect the fact that in Grenada there are automatic wage increments that are not linked to performance assessments.

\footnotetext{
${ }^{4}$ Interestingly, public sector wage demonstration effects are also known to be larger in countries with highly volatile private sector employment (see, for example, Demekas and Kontolemis, 1999) and therefore the cyclicality of the tourism sector could have aggravated this effect.

${ }^{5}$ The regression uses macro-level data within a standard VAR framework, in which nominal public and private-sector wages are jointly determined in the presence of endogenous variables, such as prices (inflation indexation) and productivity. In addition, a conditional Granger causality test is performed to confirm the causal relations between these variables. With only annual data available and relatively limited time series, the lag length is set to 1 for the regression.

$Y_{-} t=C+\Sigma_{-}(j=1)^{\wedge} p_{\text {in: }} B_{-} j Y_{-}(t-j)+\varepsilon_{-} t$

Where $Y_{-} t$ is the vector of endogenous variables (wPub, wPrv, $P$, and A); wPub denotes average wage in the public sector, wPrv average wage in the private sector, $\mathrm{P}$ consumer price index, and A total economy labor productivity (real output per worker).
} 


\section{The case of high unemployment in Grenada also illustrates the impact of natural disasters and sectoral shifts on increasing temporary and structural unemployment,} respectively. This island country was hit in 2004 by Hurricane Ivan and again by Hurricane Emily in 2005, which considerably affected the labor-intensive agricultural industry. In addition, agriculture has been experiencing a trend contracion since the mid 1990s, partly reflecting the decline in the banana industry ${ }^{6}$. These two factors contributed to the halving of formal agricultural employment (measured in NIS statistics) between 2001 and 2008, which considerably affects total employment since agriculture accounted for 12 percent of total employment in 2001. However, not all natural disasters affect employment similarly as, for example, the 2010 Hurricane Thomas in St. Lucia did not have a significant affect on employment, because the storm was less severe and agricultural employment accounts for a smaller share of total employment in St. Lucia.

\section{There are other factors, which in theory and according to anecdotal reports, could} contribute to high unemployment in these countries and need to be further explored ${ }^{7}$. The following factors could be fueling structural unemployment in St. Lucia and Grenada:

- High reservation wages due to high paid jobs in tourism and the public sector ${ }^{8}$. According to NIS statistics, the average salary in 2013 in the tourism and public sector in Grenada was 63 and 90 percent higher than in formal agriculture and 50 percent and 63 percent higher in St. Lucia. This could provide economic support for the significant segment of Grenada's labor force that are unemployed but not searching for a job, thus boosting structural unemployment ${ }^{9}$. Higher wages in the tourism industry could also have a seasonal component as the high, relatively well-remunerated employment in tourism during the high season could also fuel unemployment discouraging employment search or entrepreneurship during the low tourism season ${ }^{10}$.

- The high level of remittances to the region could also contribute to higher unemployment by increasing the "reservation wage". There is a significant ECCU

\footnotetext{
${ }^{6}$ The decline of what was once a buoyant banana industry in ECCU's Windward Islands (Dominica, Grenada, St. Lucia, and St. Vincent and the Grenadines) since the mid 1990s has also affected employment. This decline was prompted by the loss of preferential access to UK market, falling export prices, severe damages from hurricanes, as well as pest and disease.

${ }^{7}$ Further labor survey analysis in St. Lucia and Grenada will be presented in a forthcoming IMF WP to determine the significance of these factors.

${ }^{8}$ In fact, there are reports that young unemployed workers neglect taking jobs in agricultural despite the high youth unemployment recorded in the region.

${ }^{9}$ Preliminary probit analysis on the 2015 Labor Force Survey in Grenada indicates that there is a positive link between having a head of the household working in tourism or the public sector and having a member of the household unemployed and not searching for a job although with weak statistical significance. This hypothesis needs to be tested with a larger sample size and preferably using search-matching models.

10 Note though that the impact of seasonality appears only moderate in St. Lucia, the only country with quarterly labor survey data, where unemployment in 2nd and 3rd quarters (low season) of 2014 was around 25 percent, and in 1 st and 4th quarters (high season) of that year around 23 percent.
}

(continued) 
diaspora mainly in Canada, UK and the US, which provides considerable remittance flows to the region. According to Grenada's 2015 Labor Force Survey, 14 percent of Grenadian households receive remittances from abroad ${ }^{11}$.

\section{Given the recent economic stagnation in these countries, fostering wage moderation} and productivity growth could clearly be an effective path to reduce the high unemployment levels in ECCU countries. With unemployment apparently aggravated by widening wageproductivity gap in St. Lucia and the possible existence of high reservations wages in some countries, the most effective and socially acceptable way to lower unemployment is to boost productivity growth while restraining the growth of wages. In this context, staff's traditional fiscal policy advice to contain the public sector wage bill would also seem to be important to support employment growth in the private sector. As mentioned in this staff report, fostering productivity requires increasing economies scale in these small islands through measures to increase international and intraregional integration, improving the investment climate, lowering the cost of energy through investment in renewable sources, and setting up labor training programs and an education system in tune with labor market demand.

\footnotetext{
11 Preliminary probit analysis finds a positive though statistically weak correlation between the receipts of remittances and the probability of being unemployed. This hypothesis needs to be tested with a larger sample size and controlling for reverse causality.
} 


\section{Annex X. ECCU Energy: Macro-Related Challenges ${ }^{1}$}

High energy costs contribute to dampening ECCU competitiveness and potential growth. To move the energy agenda forward in line with analyses of the macro-aspects of energy reform this appendix provides: (i) a quantitative assessment of the impact of energy costs on growth and competitiveness; ii) an evaluation of gains from implementing announced renewable energy and energy efficiency targets; and iii) an analysis of the impact of energy investments on debt sustainability.

\section{The cost of electricity in the ECCU has been persistently high over the past two}

decades, and has eroded competitiveness. This is largely due to serious inefficiencies in the power sector and dependence on expensive imported petroleum products. Figure 1 shows the very high reliance of ECCU countries on diesel/oil sources, significantly higher than the LAC average. This, in turn, has contributed to the region's high cost of doing business, has increased external sector vulnerabilities, and has undercut growth. A large percent of firms in Dominica, St. Kitts and Nevis, and St. Lucia cite electricity as a major constraint to their operations (see Figure 2).
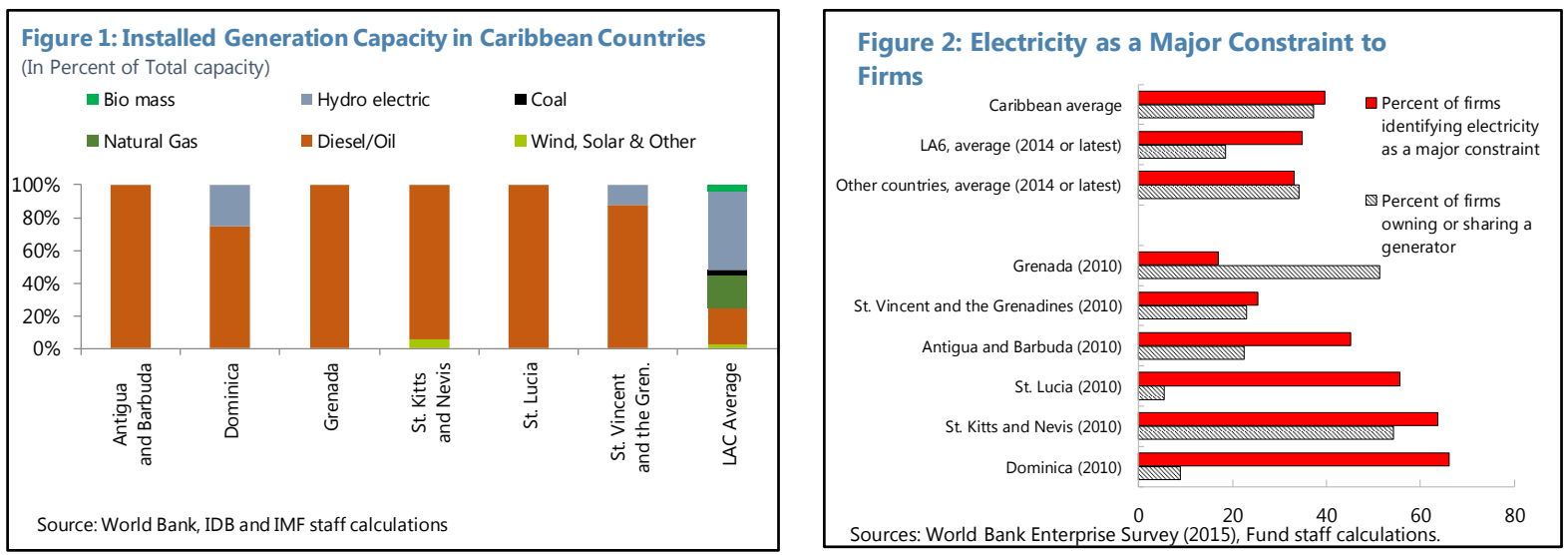

\section{Quantitative analysis suggests that} reducing high energy costs would have a discernible growth impact-though short of offering a panacea. Staff estimates suggest that around 7.8 percent of GDP variation in ECCU countries can be explained by oil price changes (see Figure 3), and that a 10 percent improvement in energy efficiency in Caribbean countries would leave long-run GDP 2 percent higher. Hence, although other factors may weigh more heavily

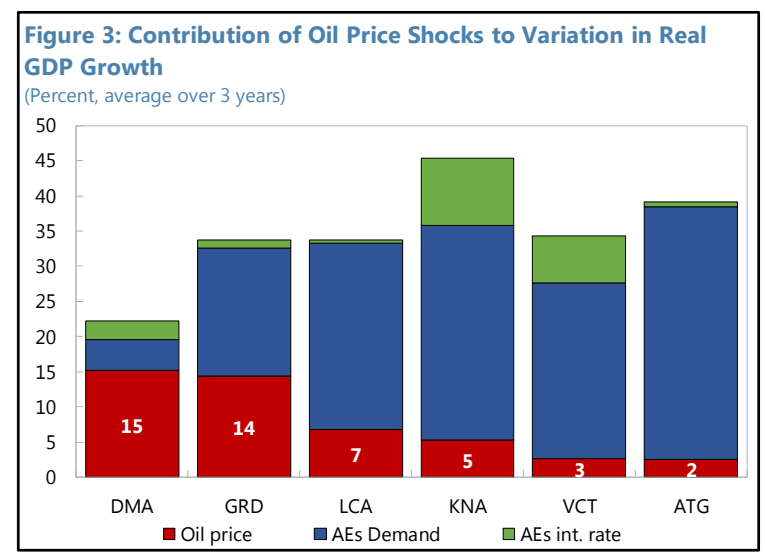

\footnotetext{
${ }^{1}$ Prepared by Gonzalo Salinas. Based on McIntyre et al, 2016, "Caribbean Energy: Macro-Related Challenges," IMF
} WP/16/53. 
on regional growth, energy sector reform could improve the region's growth outcome.

3. Most countries have drawn up national energy strategies-albeit with regulatory gaps-broadly aligned with CARICOM's regional strategy. ECCU countries have significant potential to develop renewable energy (see Table 1). The strategies set specific targets for diversifying the generation mix and improving energy efficiency in the power sector (see Tables 2 and 3), but would benefit from further development on the regulatory side. Staff estimates indicate that implementing these targets could generate significant cost savings, through lowering electricity tariffs and fuel import costs—and hence ultimately support growth (See Figure 4).

\begin{tabular}{|c|c|c|c|c|c|c|}
\hline Country & $\begin{array}{l}\text { Hydro } \\
\text { (MW) }\end{array}$ & $\begin{array}{l}\text { Wind } \\
\text { (MW) }\end{array}$ & $\begin{array}{l}\text { Geothermal } \\
\text { (MW) }\end{array}$ & $\begin{array}{l}\text { Solar PV } \\
\text { (MW) }\end{array}$ & $\begin{array}{l}\text { Biomass } \\
\text { (MW) }\end{array}$ & $\begin{array}{l}\text { Total Potential } \\
\text { (MW) }\end{array}$ \\
\hline Antigua and Barbuda & NA & 400 & NA & 27 & Unknown & 427 \\
\hline Dominica & 17 & 30 & 300 & 45 & Unknown & 392 \\
\hline Grenada & 0.5 & 5 & 50 & Unknown & Unknown & 55.5 \\
\hline St. Kitts and Nevis & NA & 5 & 300 (Nevis) & 16 & 10 & 331 \\
\hline St. Lucia & 0.2 & 40 & 170 & 36 & Unknown & 246.2 \\
\hline St. Vincent and the Grenadines & 10 & 8 & 100 & 23 & 4 & 145 \\
\hline Total & 27.7 & 488 & 620 & 147 & 14 & 1597 \\
\hline
\end{tabular}

\begin{tabular}{|l|c|c|}
\hline \multicolumn{3}{|c|}{ Table 2: Renewable Energy Targets in ECCU Countries } \\
\hline \multicolumn{1}{|c|}{ Country } & $\begin{array}{c}\text { Renewable Energy } \\
\text { Supply }\end{array}$ & Electricity from Renewables \\
\hline Antigua and Barbuda & $15 \%$ by 2030 & $20 \%$ by 2020 \\
\hline Dominica & $100 \%$ by 2020 & $\begin{array}{c}25 \% \text { by } 2010,100 \% \text { through the addition of } \\
\text { geothermal by } 2020\end{array}$ \\
\hline Grenada & $20 \%$ by 2020 & $\begin{array}{c}10 \% \text { by } 2013 \text { (Grenada), } 20 \% \text { by } 2017 \\
\text { (Grenada), } 40 \% \text { by } 2011 \text { (Carriacou and Petite } \\
\text { Martinique), } 100 \% \text { by } 2030 \text { (no date given) }\end{array}$ \\
\hline St. Lucia & $35 \%$ by 2020 & $\begin{array}{c}5 \% \text { by } 2013 ; 15 \% \text { by } 2015 \\
30 \% \text { by } 2020\end{array}$ \\
\hline St. Kitts and Nevis & & $20 \%$ by 2015 \\
& & $300 \%$ by 2010 (Nevis) \\
\hline St. Vincent and the & & $30 \%$ by 2015 \\
Grenadines 2020
\end{tabular}

\begin{tabular}{|l|c|}
\hline \multicolumn{2}{|c|}{ Table 3: National Energy Efficiency Targets in ECCU Countries } \\
\hline \multicolumn{1}{|c|}{ Country } & Energy Efficiency \\
\hline Antigua and Barbuda & Improve energy efficiency 20\% by 2020 \\
\hline Dominica & Reduce public sector electricity consumption 20\% by 2020 \\
\hline Grenada & Reduce public sector electricity consumption 20\% by 2020 \\
\hline St. Lucia & Reduce projected electricity demand 20\% by 2015 (resulting \\
\hline St. Kitts and Nevis & Reduce projected increase in peak demand 5\% by 2015 and \\
\hline $\begin{array}{l}\text { St. Vincent and the } \\
\text { Grenadines }\end{array}$ & \\
\hline & \\
\hline
\end{tabular}

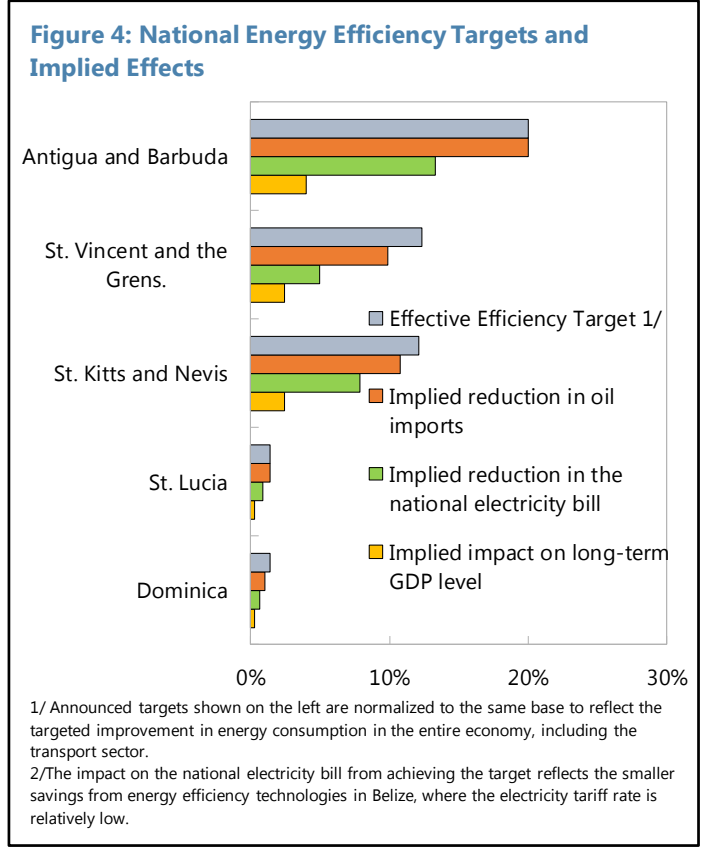


4. The upfront investment costs associated with countries' energy strategies are substantial but in most cases not insurmountable (See Table 4). Broad-brush efforts at quantifying the investment envelope required to implement countries' energy strategies suggest a regional average cost in the order of 7 percent of estimated 2015 GDP. While significant, such investment bills may still be within the reach of some countries in the region without jeopardizing public debt sustainability or-preferably-could be covered by bringing in private investment.

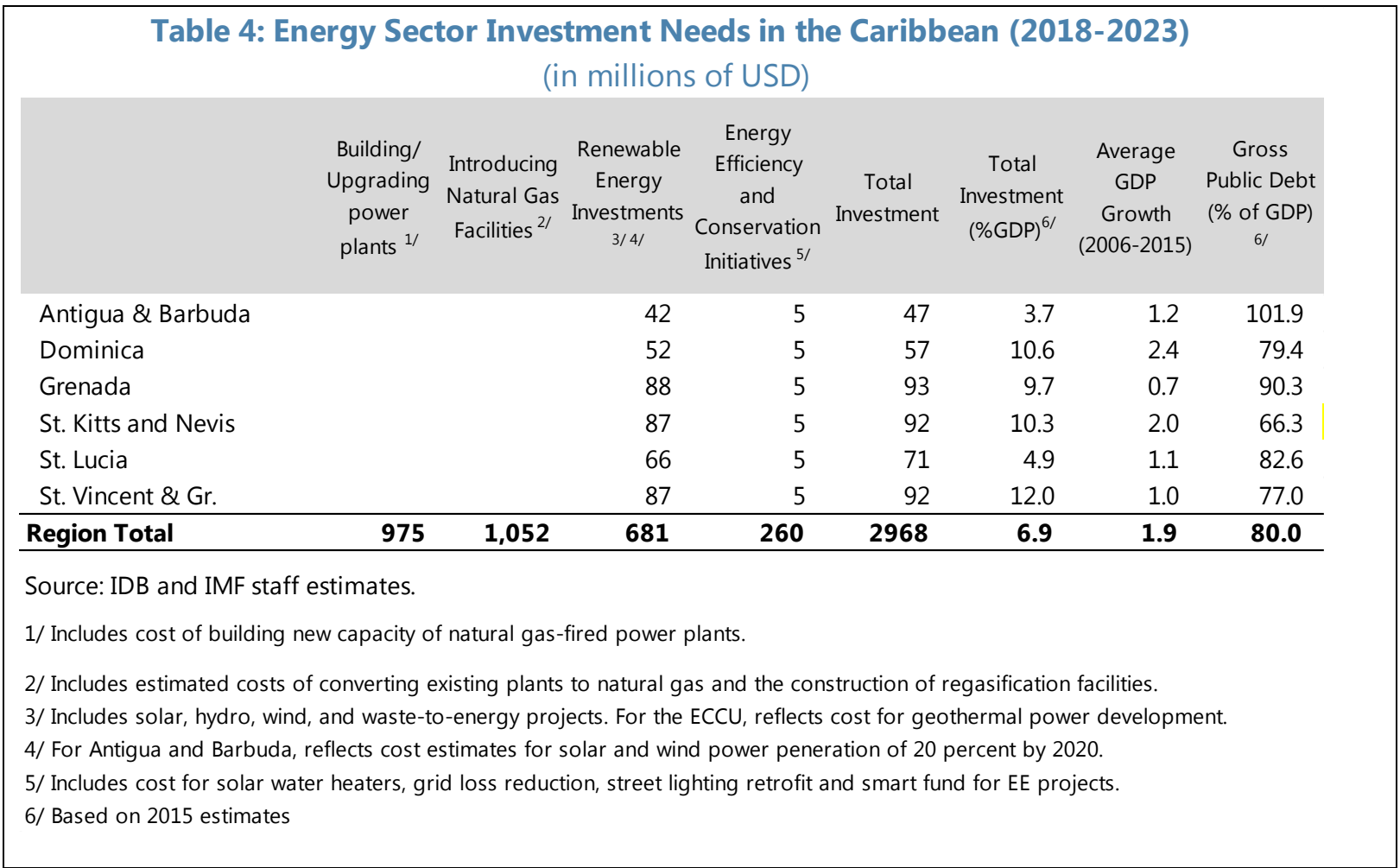

5. Public financing of energy investments is likely to be feasible only in countries with sustainable debt dynamics and reasonably low risk of debt distress. ECCU countries where fiscal vulnerabilities remain serious need to ensure that undertaking energy investments does not alter their long-run public debt trajectory or derail necessary fiscal adjustment efforts over the medium term. To achieve this, investments need to be high-yielding - to provide a sufficient rate of return to be self-financing. Figure 6 shows the estimated impact of energy investment on debt sustainability in ECCU countries.

6. A bigger role for the private sector, including through public-private partnerships, would alleviate sustainability pressures from energy investment. But a strong operational and institutional framework is a prerequisite to safeguard against contingent liability risks and ensure that project implementation follows best practices.

7. To move the energy agenda forward, policymakers should put in place the regulatory and legislative reforms needed for an enabling policy environment. To foster sustainable and affordable energy solutions, measures are needed to facilitate the introduction of alternative energy 
sources, including clear procedures for licensing Independent Power Producers (IPPs) and integrating them in the power sector through net-metering and net-billing schemes. Strengthening institutional capacity by creation of independent national and/or regional energy sector regulators will also help promote a predictable and transparent regulatory environment in the region. And establishing national energy efficiency standards (e.g. energy labeling and energy efficient building codes) will encourage the adoption of energy-efficient technologies by households and businesses, particularly hotels. 


\section{Figure 6. Impact of Energy Investments on Debt Sustainability ${ }^{1 /}$}

Antigua and Barbuda: Public Debt

(In percent of GDP)

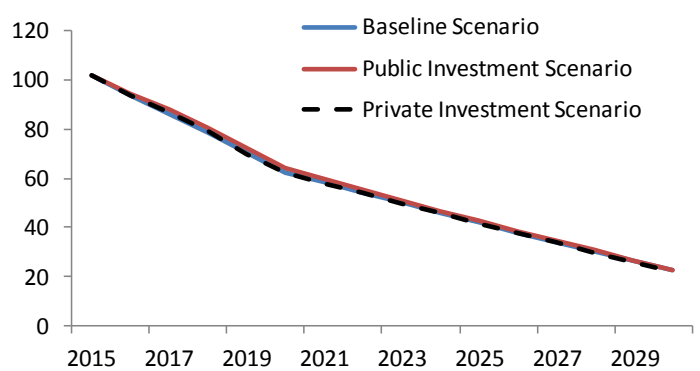

Grenada: Public Debt

(In percent of GDP)

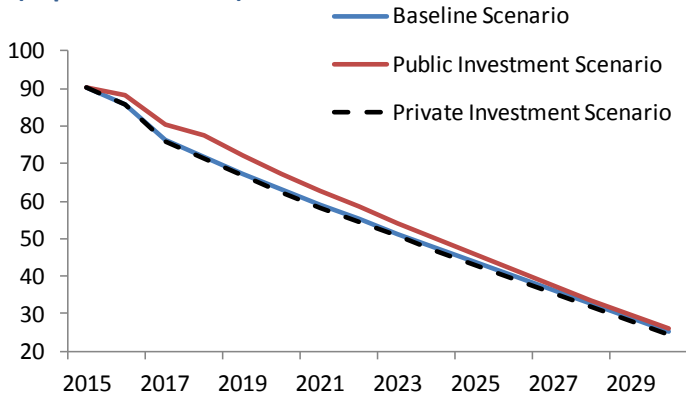

St. Lucia: Public Debt

(In percent of GDP)

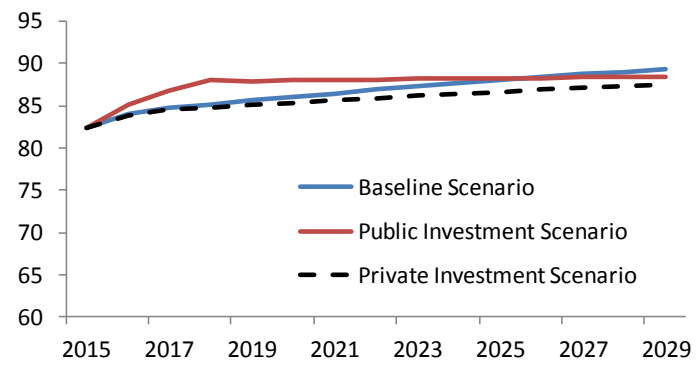

Dominica: Public Debt

(In percent of GDP)

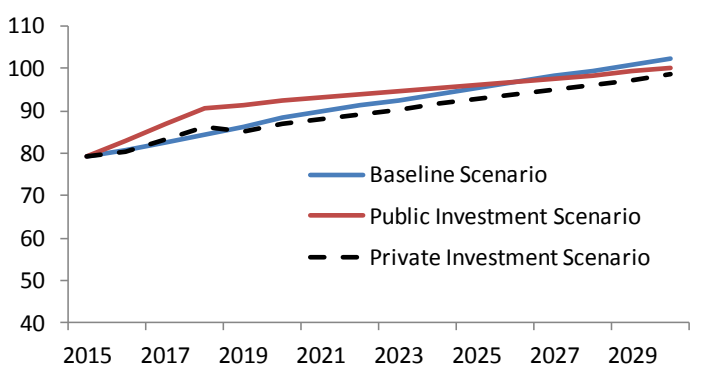

St. Kitts and Nevis: Public Debt (In percent of GDP)

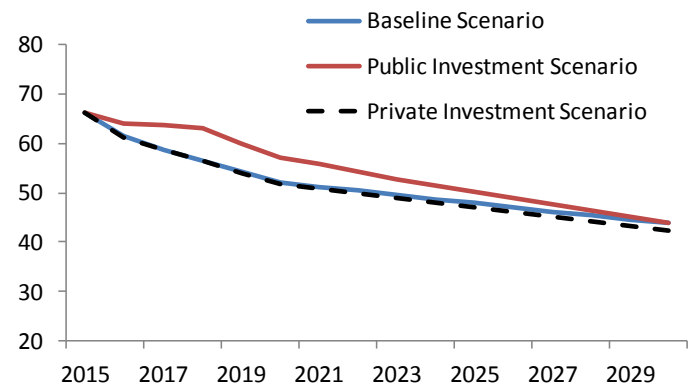

St. Vincent and the Grenadines: Public Debt (In percent of GDP)

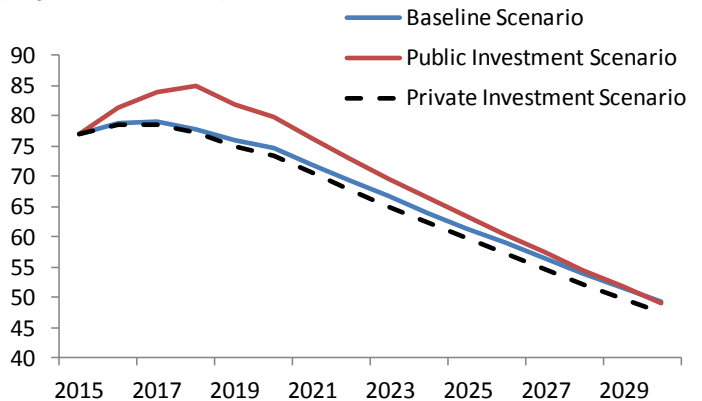




\section{Annex XI. United Kingdom Overseas Territories-Anguilla and Montserrat ${ }^{1}$}

\section{Anguilla}

\section{Like the rest of the ECCU, a positive global environment is supporting a modest} economic recovery. Growth in 2015 was 2.2 percent, underpinned by a growth in tourism arrivals of 5.2 percent. This has been followed by a successful 2016 winter season in which stay-over arrivals rose by 7.7 percent compared with the previous year. However, ongoing delays in resolving the two insolvent banks under ECCB conservatorship continued to be a drag on growth, as bank credit declined by almost 4 percent, the fourth consecutive year of credit contraction.

\section{The resolution of the banks in April 2016 has come at a large fiscal cost that will require a substantial fiscal adjustment in order to restore fiscal and debt sustainability.} Anguilla's 2014 fiscal framework established ceilings on net debt and annual debt service of 80 percent of recurrent revenue and 10 percent of recurrent revenue, respectively to be achieved by 2017. The gross fiscal cost of the bank resolution-some 40 percent of GDP in new government debt-made those targets unattainable and required a waiver of the fiscal rules. ${ }^{2}$ The U.K. agreed to the waiver based on the Anguillan authorities' commitment to a medium-term fiscal adjustment and economic development program that would achieve the fiscal targets no later than 2028 and to assurances that reforms would be implemented to substantially strengthen bank supervision and regulation.

\section{Staff's medium-term projections assume the implementation of the authorities' plan.} Growth is projected to gradually rise to 2.7 percent in the medium term, based on the continued growth of tourism, including as a result of reforms to improve competitiveness; a return to normalcy in the banking system, and the adoption of fiscal adjustment measures.

\section{Total fiscal measures of close to 3 percent of GDP are envisaged over 2016-17 raising} the primary surplus from 3.2 percent of GDP in 2015 to 5.9 percent in 2020. In the 2016-17 budget, fiscal measures of $2 \frac{1}{2} 2$ percent of GDP were enacted, including the introduction of new fees on petroleum importers; an increase in existing fees (e.g., land registry, driver's and vehicle licenses); the introduction of gross revenue taxes on petroleum importers and the electricity company, ANGELEC; and an increase in property taxes based on a new valuation methodology. The government also plans to divest half of its 40 percent stake in ANGELEC. In 2017, the government plans a slightly revenue-positive tax reform, including the introduction of a general sales tax at 15 percent (expected yield 7.5 percent of GDP), offset by a reduction in import duties and the elimination of the accommodation tax and other minor taxes. The government also intends to

\footnotetext{
1 Prepared by Alla Myrvoda and Gonzalo Salinas.

2 The net fiscal cost, i.e., net of recoveries of NPLs, is estimated to be either 31 percent of GDP, if offshore placements in the resolved banks are ruled to have been deposits or 25 percent of GDP if the placements are ruled to not have been deposits.
} 
introduce a social development levy to fund health and education, which will replace the stabilization levy (income tax).

5. To improve competitiveness and support growth, the authorities expect to receive close to US\$60 million (20 percent of GDP) in capital grants from the U.K. government to fund much needed infrastructure. Upgrading the two main entry points into the island (the ferry terminal and international airport) will be critical to catalyze new hotel investment and a planned expansion of tourism activity. The authorities' economic development strategy also contemplates diversification into new economic areas, including fisheries, which would take advantage of Anguilla's 200 nautical mile Exclusive Fishing Zone, renewable energy, and an economic residency program.

\section{Montserrat}

6. Capital expenditure reprioritization in 2015, which triggered an improvement in fiscal indicators but slowed economic growth, is expected to provide a boost to the economy over the medium-term. Given the significance of the public sector to economic development, real GDP growth in 2015 is estimated to have slowed to $1 / 2$ percent year-on-year largely due to the reassessment and rebalancing of the capital investment objectives of Montserrat. This led to a decline in spending during the reprioritization process but is expected to provide a boost to economic growth in the medium-term. The reprioritization of capital investments also resulted in a substantial improvement in the fiscal accounts in 2015, with the overall balance shifting from a deficit of close to 6 percent of GDP to a surplus of the same amount. Public debt remains well below international averages at 7.7 percent of GDP. While credit to private sector growth continued to recover since late 2014, reaching 7.2 percent in February 2016, deposit accumulation decelerated from 12 percent year-on-year growth at end-2014 to about 2.5 percent in February 2015. The external position has improved slightly as the current account deficit is estimated to have narrowed, reflecting lower oil and commodity prices (Table 2 ).

\section{Montserrat's economic outlook hinges on the advancements made with major public}

capital projects. Over the medium term, economic growth is projected to average about 2.2 percent of GDP. Social programs to improve health and education quality and infrastructure, combined with capital projects to support and enhance tourism, energy, and construction are key to unlocking growth. Efforts continue to increase access to social housing, upgrade health and educational infrastructures, and to improve social and human development. In addition to social structures, the authorities are implementing a number of infrastructure projects aimed at improving roads and bridges, upgrading electricity distribution, water, and waste management systems. The construction of a new port is also critical to unlocking greater economic potential through improving cruise tourism and connectivity to the island. A resumption of strong, sustainable growth will be key to reduce reliance on UK grants, which finance about 60-70 percent of total expenditures.

8. The abundance of renewable energy resources, namely solar, geothermal, and wind, has given the island the ability to implement a new energy policy, which aims to put Montserrat on a 100 percent renewable energy generation by 2020. This will allow Montserrat 
to benefit from cheaper electricity, given that currently electricity charges constitute a significant expense for businesses and households alike. Thus, in FY2016-17, in addition to exploring the feasibility of the use of solar energy, the authorities intend to continue advancing toward geothermal energy by drilling a third well, which is expected to raise the island's potential further. Lower energy costs, combined with easier access to the island by tourism, can create incentives for investment and boost commercial development on the island, and increase private sector participation. 
Table 1. Anguilla: Selected Indicators, 2011-17

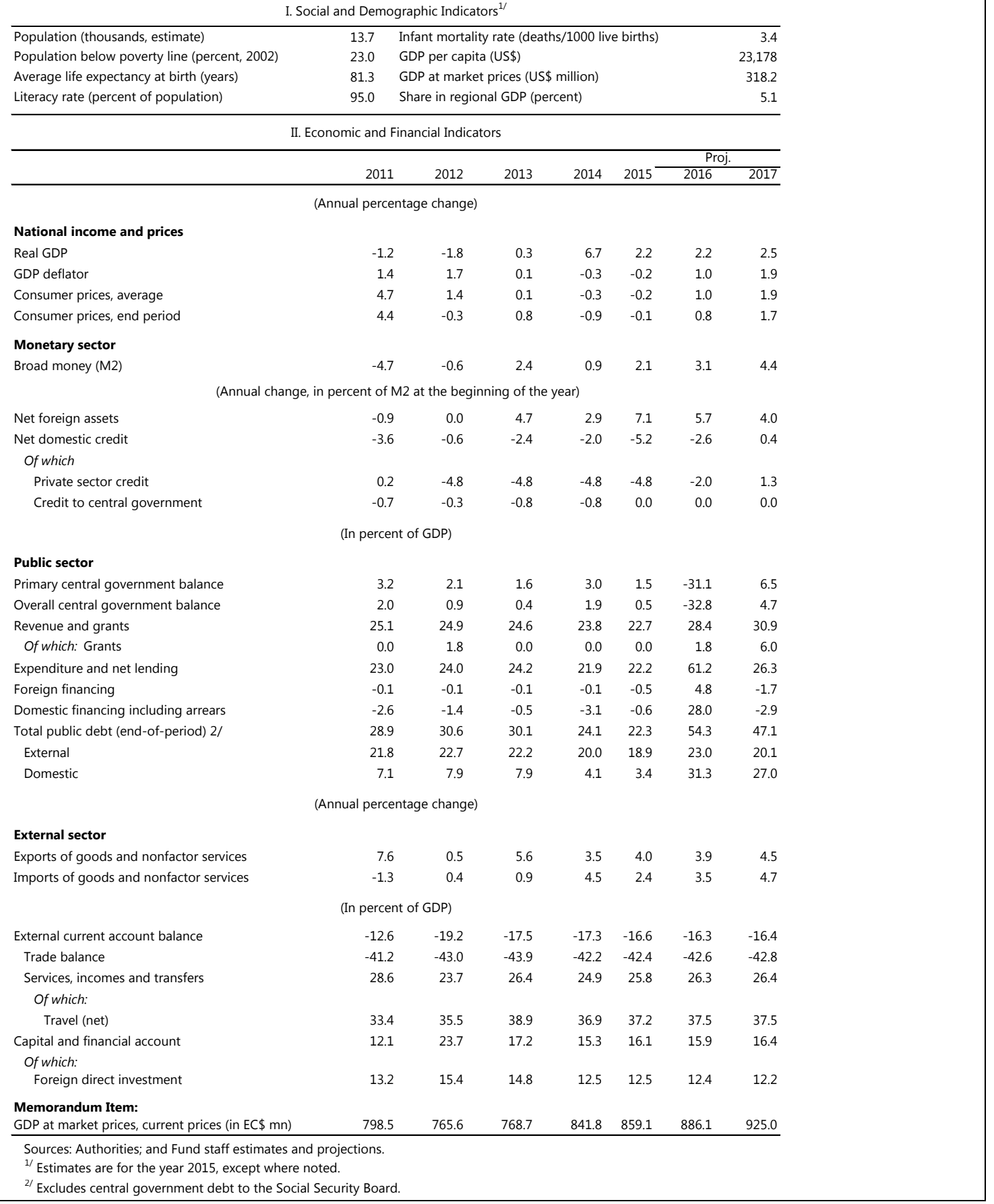

\section{CInternational Monetary Fund. Not for Redistribution}


Table 2. Montserrat: Selected Indicators, 2011-17

I. Social and Demographic Indicators $1 /$

\begin{tabular}{lrlr}
\hline Population (thousands, estimate) & 5.2 & Gini coefficient & 0.3 \\
Average life expectancy at birth (years) & 73.2 & GDP per capita (US\$), estime & 11,893 \\
Literacy rate (percent of population) & 97.0 & GDP at market prices (US\$ mln) & 61.8 \\
Infant mortality rate (deaths / 1000 live births) & 15.8 & Share in regional GDP (percent) & 1.1
\end{tabular}

II. Economic and Financial Indicators

\begin{tabular}{|c|c|c|c|c|c|c|c|}
\hline & \multirow[b]{2}{*}{2011} & \multirow[b]{2}{*}{2012} & \multirow[b]{2}{*}{2013} & \multirow[b]{2}{*}{2014} & \multirow{2}{*}{$\begin{array}{l}\text { Est. } \\
2015\end{array}$} & \multicolumn{2}{|c|}{ Proj. } \\
\hline & & & & & & 2016 & 2017 \\
\hline \multicolumn{8}{|c|}{ (Annual percentage change) } \\
\hline \multicolumn{8}{|l|}{ National income and prices } \\
\hline Real GDP & 6.2 & 1.6 & 2.8 & 1.3 & 0.5 & 1.3 & 1.7 \\
\hline GDP deflator & 5.3 & -1.5 & 0.9 & -0.3 & -1.1 & 0.1 & 0.7 \\
\hline Consumer prices, average & 3.7 & 4.8 & 0.9 & -0.3 & -1.1 & 0.1 & 0.7 \\
\hline Consumer prices, end-period & 4.4 & 3.6 & -0.5 & -1.0 & -0.1 & 0.1 & 0.8 \\
\hline \multicolumn{8}{|l|}{ Monetary sector } \\
\hline Broad money (M2) & 13.0 & 14.7 & -2.5 & 11.1 & 2.4 & 3.0 & 2.5 \\
\hline \multicolumn{8}{|c|}{ (Annual change, in percent of $\mathrm{M} 2$ at the beginning of the year) } \\
\hline Net foreign assets & 26.5 & 25.7 & -4.0 & 8.4 & 13.6 & 2.5 & 1.6 \\
\hline Net domestic assets & -13.6 & -11.0 & 1.5 & 2.7 & -11.2 & 0.4 & 0.8 \\
\hline \multicolumn{8}{|l|}{ Of which: } \\
\hline Private sector credit & 3.9 & 3.5 & -3.8 & 0.1 & 1.5 & 2.5 & 1.7 \\
\hline
\end{tabular}

(In percent of GDP)

Public sector

Primary central government balance Overall central government balance

$\begin{array}{rrrrrrr}7.7 & -6.9 & -16.7 & -5.8 & 6.0 & 3.1 & 1.2 \\ 7.7 & -6.9 & -16.8 & -5.8 & 5.9 & 2.9 & 1.0 \\ 78.0 & 81.4 & 94.3 & 89.4 & 106.9 & 97.6 & 96.4 \\ 54.0 & 56.6 & 68.8 & 62.4 & 78.8 & 69.7 & 68.1 \\ 70.3 & 88.4 & 111.1 & 95.2 & 101.0 & 94.7 & 95.4 \\ -0.1 & -0.1 & -0.1 & -0.1 & -0.1 & -0.1 & -0.1 \\ -7.6 & 7.0 & 16.8 & 5.9 & -5.9 & -2.8 & -0.9 \\ 14.2 & -8.5 & -21.4 & -1.6 & 3.3 & 2.9 & 1.0 \\ 4.7 & 4.2 & 4.6 & 4.1 & 7.6 & 7.1 & 6.6\end{array}$

Revenue and grants

Of which: Grants

Expenditure and net lending

Foreign financing

Domestic financing including arrears

Central government current account balance

Total public debt (end-of-period)

(Annual percentage change)

External sector

Exports of goods and nonfactor services

Imports of goods and nonfactor services

\begin{tabular}{rrrrrrr}
18.0 & 4.9 & 25.8 & -8.5 & 3.1 & 2.4 & 3.5 \\
12.7 & 8.0 & 30.7 & -10.1 & 2.2 & -9.6 & 8.0 \\
(In percent of GDP) & & & & & & \\
-15.8 & -21.4 & -48.0 & -29.9 & -27.3 & -13.0 & -19.4 \\
-42.8 & -48.8 & -51.8 & -44.4 & -43.8 & -32.6 & -36.0 \\
27.0 & 27.4 & 3.8 & 14.4 & 16.5 & 19.6 & 16.5 \\
& & & & & & \\
8.1 & 11.1 & 11.2 & 11.4 & 11.9 & 12.1 & 12.3 \\
26.0 & 30.7 & 64.6 & 35.6 & 32.8 & 17.5 & 23.0 \\
& & & & & & \\
3.9 & 4.1 & 6.2 & 5.9 & 6.3 & 6.3 & 6.3 \\
& & & & & & \\
172.0 & 171.3 & 163.2 & 168.1 & 167.0 & 169.3 & 173.5 \\
\hline
\end{tabular}

External current account balance

Trade balance

Services, incomes and transfers

Of which:

Travel (net)

Capital and financial account

Of which:

Foreign direct investment

Memorandum item:

GDP at market prices, current prices (in EC $\$ \mathrm{mn}$ )

Sources: Authorities; ECCB; and Fund staff estimates and projections.
1 - Estimates are for the year 2013, except where noted. Balance of payments 2014 onwards projections (BPM5 methodology).

CInternational Monetary Fund. Not for Redistribution 


\section{Annex XII. External Assessment in the ECCU 1}

\section{Current account based REER misalignment estimates are substantially affected by a} recent revision in external sector statistics (Table 1). The authorities are in the process of migrating balance of payments statistics from BPM5 to BPM 6 methodology, which has partly resulted in a significant increase in measured tourism receipts. Preliminary BPM6 based estimates present major improvements in current account balances in all countries in the region in 2104, as seen the first column of Table 1. As a result, the weighted average ECCU current account deficit has been revised down from 13 to 3 percent of GDP, which considerably affects current account based REER assessments. In particular, the current account EBA-Lite analysis using preliminary BPM6 data results in an average REER undervaluation (8.8 percent in weighted-average terms) compared with results using the BPM5 data (around 32 percent). There is though considerably variation around this mean with only Antigua and Barbuda and Grenada showing only moderate REER misalignments using BPM6 data. ${ }^{23}$

\begin{tabular}{|c|c|c|c|c|c|c|}
\hline & Actual CA & CA Norm & $\begin{array}{c}\text { Policy C.A. } \\
\text { Gap }\end{array}$ & Residual & $\begin{array}{l}\text { Exchange } \\
\text { Rate } \\
\text { Elasticity }\end{array}$ & $\begin{array}{l}\text { REER Gap/ } \\
\text { Misalignment }\end{array}$ \\
\hline \multicolumn{7}{|l|}{ BPM5 Current Account } \\
\hline Antigua and Barbuda & -14.5 & -3.1 & 1.5 & -12.9 & -0.3 & 42.6 \\
\hline Dominica & -13.1 & -27.9 & 0.9 & 14.0 & -0.3 & -72.9 \\
\hline Grenada & -15.5 & -6.8 & 1.7 & -10.4 & -0.2 & 51.0 \\
\hline St. Kitts and Nevis & -7.6 & -6.9 & 8.5 & -9.2 & -0.2 & 2.8 \\
\hline St. Lucia & -6.7 & -5.0 & 8.5 & -2.1 & -0.3 & 6.3 \\
\hline St. Vincent and the Grenadines & -29.6 & -7.3 & 2.8 & -25.0 & -0.1 & 167.5 \\
\hline $\mathrm{ECCU}$, simple average & -14.5 & -9.5 & 4.0 & -7.6 & -0.2 & 32.9 \\
\hline $\mathrm{ECCU}$, weighted average & -12.7 & -7.1 & 4.2 & -7.8 & -0.2 & 32.2 \\
\hline \multicolumn{7}{|c|}{ BPM6 Current Account (Provisional) } \\
\hline Antigua and Barbuda & -1.7 & -3.1 & 1.5 & -0.1 & -0.3 & -5.3 \\
\hline Dominica & -6.8 & -27.9 & 0.9 & 20.2 & -0.3 & -103.3 \\
\hline Grenada & -7.8 & -6.8 & 1.7 & -2.7 & -0.2 & 5.8 \\
\hline St. Kitts and Nevis & 3.0 & -5.6 & 7.2 & 1.4 & -0.2 & -36.3 \\
\hline St. Lucia & 7.2 & -5.0 & 7.2 & 11.7 & -0.3 & -43.8 \\
\hline St. Vincent and the Grenadines & -24.6 & -7.3 & 2.8 & -20.0 & -0.1 & 129.9 \\
\hline ECCU, simple average & -5.1 & -9.3 & 3.5 & 1.8 & -0.2 & -8.8 \\
\hline $\mathrm{ECCU}$, weighted average & -3.0 & -6.9 & 3.6 & 1.8 & -0.2 & -8.8 \\
\hline
\end{tabular}

\footnotetext{
1 Prepared by Gonzalo Salinas.

2 The reserve adequacy metric for small islands, based on IMF WP/12/205, takes into account the higher precautionary motive of these countries due to several factors including natural disasters.

${ }^{3}$ The ratio of reserves to the 12-month forward-looking debt service plus the current account deficit is 1.63.
} 


\section{An Index-based EBA-Lite assessment implies there are only moderate REER}

misalignments in most ECCU countries (Table 2). This methodology, which is based on cross country regressions that directly use estimated REER as dependent variable, actually points to a moderate REER undervaluation in average. The range across estimates by countries is much narrower than under the current account based EBA-Lite, with St. Kitts showing the most overvalued REER (10.9 percent) and Grenada showing the most undervalued REER (12.3 percent).

\begin{tabular}{|c|c|c|c|}
\hline \multicolumn{4}{|c|}{ Table 2. EBA-Lite: REER Index Model Estimates } \\
\hline & REER Gap & Policy Gap & Residual \\
\hline Antigua and Barbuda & -9.6 & -0.7 & -9.0 \\
\hline Dominica & -12.1 & -0.4 & -11.7 \\
\hline Grenada & -7.1 & 0.2 & -7.2 \\
\hline St. Kitts & 11.7 & -0.3 & 12.1 \\
\hline St. Lucia & 3.0 & -2.1 & 5.1 \\
\hline St. Vincent & 2.8 & -0.5 & 3.2 \\
\hline ECCU, simple average & -1.9 & -0.6 & -1.3 \\
\hline ECCU, weighted average & -1.4 & -0.7 & -0.7 \\
\hline & nd o sloulat & & \\
\hline
\end{tabular}




\section{INTERNATIONAL MONETARY FUND}

\section{EASTERN CARIBBEAN CURRENCY UNION}

STAFF REPORT FOR THE 2016 DISCUSSION ON COMMON POLICIES OF MEMBER COUNTRIES-INFORMATIONAL ANNEX

Prepared By

The Western Hemisphere Department

(In collaboration with other departments)

\section{CONTENTS}

RELATIONS WITH THE FUND $\underline{\mathbf{2}}$ 


\section{RELATIONS WITH THE FUND}

(As of June 14, 2016)

Membership Status: Not Applicable

\section{Exchange Arrangement:}

The Eastern Caribbean Currency Union (ECCU) comprises six Fund members: Antigua and Barbuda, Dominica, Grenada, St. Kitts and Nevis, St. Lucia, and St. Vincent and the Grenadines; and two territories of the United Kingdom, Anguilla and Montserrat. The eight ECCU members have a common currency, monetary policy, and exchange system. The common currency, the Eastern Caribbean (EC) dollar, has been pegged to the U.S. dollar at the rate of EC $\$ 2.70$ per U.S. dollar since July 1976. The common central bank, the Eastern Caribbean Central Bank $(E C C B)$, has operated like a quasi-currency board, maintaining foreign exchange backing of its currency and demand liabilities of close to 100 percent.

\section{Safeguards Assessment}

Under the Fund's safeguards policy, the Eastern Caribbean Central Bank (ECCB) is subject to a full safeguards assessment on a four-year cycle. An update assessment was completed in April 2016 and found that the ECCB has maintained generally strong controls over its key operations. External audit and financial reporting practices remain sound. The ECCB financial statements are compliant with International Financial Reporting Standards and are published on a timely basis. The internal audit function needs to be reformed to align it with leading international practices and oversight could be further strengthened by enhancing the financial expertise of the audit committee. 


\section{CARTAC: CAPACITY BUILDING IN THE ECCU}

The Caribbean Regional Technical Assistance Center (CARTAC) was established in November 2001 as a joint IMF-UNDP initiative, based in Barbados, to provide technical assistance (TA) and training to beneficiary countries, currently $20,{ }^{1}$ and to the ECCB. In 2011, CARTAC became one of the IMF's network of Regional Technical Assistance Centre, funded by development partners, its members, and by the IMF. A Steering Committee consisting of contributors to CARTAC, member countries, development partners, CARICOM and the CDB, provides strategic guidance, and ensures ownership and commitment. On January 1, 2017 CARTAC will enter its fifth phase of operations. CARTAC's core areas of TA and training include revenue administration, public financial management (PFM), macroeconomic programming and analysis, financial sector stability and supervision, and economic and financial statistics.

The ECCU region accounts for just over 40 percent of all TA to the CARTAC membership (Figure 1), with a particular focus on financial sector supervision, PFM, and macroeconomic programming and analysis; with 49, 45, and 44 percent respectively (Figure 2) Grenada, St. Lucia, and St. Kitts and Nevis are the most intense users of CARTAC TA amongst the ECCU countries.

Figure 1. CARTAC TA in FY2016

ECCU countries receive a considerable portion of TA...

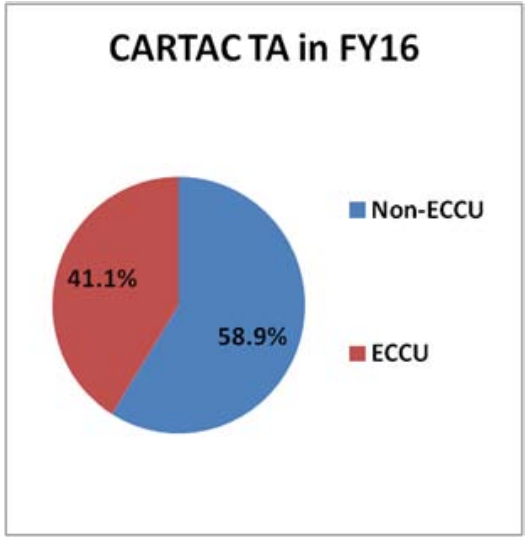

Figure 2. CARTAC Support for the ECCU Region

Financial sector, PFM and statistics account for the majority of TA...

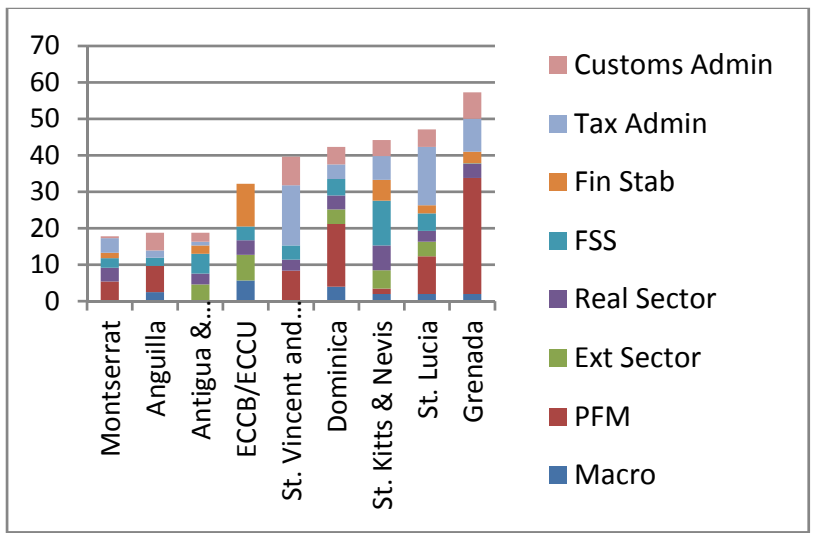

Highlights of CARTAC's TA and training to the ECCU countries in the various core areas are provided below.

In the area of revenue administration, considerable progress has been made with VAT implementation. ${ }^{2}$ The program for the ECCU member revenue agencies focuses on: (i) structural reorganization, (ii) strategic planning and performance management; (iii) capacity

\footnotetext{
${ }^{1}$ Preparations are ongoing to extend CARTAC membership to Aruba, Curacao, and St. Maarten.

2 Dominica (March 2006), Antigua and Barbuda (January 2007), St. Vincent and the Grenadines (May 2007), Grenada (February 2010), St. Kitts and Nevis (November 2010), St. Lucia (October 2012). In Anguilla in 2012, CARTAC provided support with its preparations for the introduction of a Goods and Services Tax in 2014.
} 
building in core areas (taxpayer service, audit, collection enforcement), and (iv) resource management.

On taxpayer segmentation Large and Medium Taxpayers Units (LMTUs) and Design, Planning and Monitoring Units (DPMUs) are being launched, and some are now operational. Revenue performance by this segment showed increases of 16 percent for 2015 over 2013 for St Kitts and Nevis. CARTAC reviewed the organization and operations of the St. Vincent and the Grenadines Inland Revenue Department (IRD), following which the authorities endorsed its key recommendations on modernization (including structural reorganization along functional lines; management of taxpayers by segment; and a clear separation of roles and responsibilities between operations and HQ functions). The recommendations are to be implemented on a phased basis, beginning with the establishment of the HQ function (through a planning and program design unit), and by setting up a large and medium taxpayer unit (LMTU).

Several countries are furthering their tax reform agendas, improving their institutional arrangements, and ease of administration:

- $\quad$ St. Lucia - the Corporate Income Tax (CIT) regime was reviewed; a presumptive tax was designed for implementation in FY17; the VAT threshold was increased to EC $\$ 400,000$ effective Feb.1, 2016; and advice on property tax reform was provided;

- Dominica - the CIT regime was reviewed; a presumptive tax was designed; and an increase in the VAT threshold was proposed;

- Grenada - support was provided to reform the tax incentive regime, culminating in the enactment of legislation which is now under implementation. Legislation to support the changes is yet to be fully implemented;

- $\quad$ Anguilla - a joint CARTAC/FAD tax mission responded to the government budget announcement regarding the implementation of a comprehensive fiscal reform, including the introduction of a broad-based Goods and Services Tax (GST). The mission provided advice and recommendations on indirect taxation, and other options to improve the taxation of goods and services;

- $\quad$ St. Kitts and Nevis - CARTAC provided advice on implementing and managing a tax incentive regime;

- In St. Lucia and Dominica - TA in revenue modeling was provided to enhance tax policy reforms.

A model Tax Administration Procedure Act (TAPA) has been drafted for the region to improve tax administrative effectiveness. Legislation has been enacted in Grenada (effective May 2016), and while Antigua and Barbuda has enacted a TAPA, its implementation is delayed to facilitate further discussion on the impact of the garnishment provision. Recommendations have also been made for St. Vincent and the Grenadines to similarly adopt the TAPA. 
CARTAC provided guidance through a workshop for small tax administrations on how to develop a strategic management framework, and assisted large and medium tax administrations to update their strategic plans. Corporate Strategic Plans (CSPs) updated during FY2016 included; Antigua and Barbuda and St. Kitts and Nevis. Compliance management strategies have been designed for St. Lucia, Barbados, and discussed with Grenada

Drawing on regional skills, IT support was provided to St. Lucia, Grenada, and St. Vincent and the Grenadines through enhancements to SIGTAS. ${ }^{3}$ This has helped the authorities to produce timely and more accurate reports, and to automate the setting of interest and penalties. An integrated IT solution and development of a taxpayer identification numbering system has been recommended for Montserrat.

Core functions have been strengthened to ensure improved taxpayer compliance and to reduce the tax gap. CARTAC delivered training to build capacity in core functional areas: (a) building audit capacity -St. Kitts and Nevis, (b) collection and enforcement - St. Vincent and the Grenadines; (c) property tax administration - St. Lucia; and (d) data analytics - St. Kitts and Nevis. Two regional workshops focused on collection enforcement and property tax.

Customs administration focuses on: (i) leadership, management and governance; (ii) strategic Planning; (iii) human resource development; (iv) trade facilitation; and, (v) compliance. Regional initiatives include continued support to CARICOM and the OECS in the harmonization of procedures, economic integration and free circulation of goods regime; and, in partnership with CCLEC, delivering leadership and management training.

TA has been provided to St. Kitts and Nevis and St. Vincent and the Grenadines to prepare corporate business and strategic plans, and all now have some form of corporate plan in use. In Anguilla CARTAC experts conducted organizational structure reviews and workload assessments to help the planning function.

Risk Management is the corner stone of an effective, modern customs administration. It enables customs, by gathering and analyzing information from a variety of sources, to make sound decisions about intervening in the cargo clearance process. TA has been provided to St. Lucia, and a framework has been prepared to identify, categorize and prioritize risks, and to propose risk treatments to address those risks.

TA, including advice, mentoring and training on post clearance audit (PCA), was delivered to St. Kitts and Nevis, St. Lucia, and Dominica. Approximately 20 officers received training, and as a result of this assistance, customs in these member countries now have the capacity to initiate significant PCA programs, and to strengthen both compliance and trade facilitation. Together with risk management, PCA has been an area where CARTAC has provided significant amounts of TA in FY16-to Antigua and Barbuda, Dominica, and St. Kitts and Nevis. While external factors, such as legislative constraints, have limited the ability to fully operate PCA systems,

\footnotetext{
${ }^{3}$ The Standardized Government Tax Administration System (SIGTAS) is an integrated IT system that enables government to automate the administration of all taxes and licenses using a single comprehensive information system
} 
improvements can already be seen in all of these administrations. In Dominica, approximately $\$ 700,000$ in additional duties and taxes was assessed by the PCA unit in 2015; in St. Kitts and Nevis, 13 audits were carried out in 2014/2015 with assessments of approximately $\$ 100,000$ being made.

TA, that included guidance, mentoring and training, was provided in valuation in Grenada; and training in tariff classification was provided in Dominica, Antigua and Barbuda, and St. Vincent and the Grenadines. In Grenada, additional duties and taxes assessed by customs in relation to classification and valuation has shown progressive year on year increases from US $\$ 25,000$ in 2012 to US\$120,000 in 2014. Training was provided to about 83 officers, and will mean increased consistency, fewer disputes with importers, faster clearance and, importantly, enhanced revenue collection.

In support of the planned OECS free movement of goods regime, a study and workshop to analyze trade and revenue data was delivered by CARTAC. Work was also initiated to consider revenue management options for the free circulation regime. Due to data limitations the exercise was not completed, however, sufficient analysis was possible to give OECS member governments adequate information and the confidence to press forward with the free circulation plan. CARTAC will continue to be engaged in this area, and the request of the OECS will undertake a study to provide information and possible options to help decisionmakers harmonize customs service charges.

The new international airport in St. Vincent and the Grenadines is the most significant infrastructure program in the Windward Islands in decades, and has the potential to transform the economy of those islands. To ensure that the opportunities presented by the new airport are optimized, CARTAC experts provided advice so that customs procedures give both a credible defense against smuggling, and good facilitation of tourists and other travelers. Training was provided to officers in risk assessment and control techniques, and the input will ensure that once the airport is operational, customs will play its full part.

With a view to assessing the progress in public financial management reform, CARTAC has undertaken a comparative review of PFM practices in ECCU member countries ${ }^{4}$ against overall characteristics of a "core PFM" framework ${ }^{5}$ as defined in the IMF Good Practice Note (GPN) on 'Sequencing PFM Reform' (Diamond, 2013). This analysis has produced a number of interesting findings regarding the standard of PFM across the ECCU region, and in turn informed allocation of CARTAC resources.

\footnotetext{
${ }^{4}$ Analysis was derived from the PEFA assessments conducted from 2013-2016 in Anguilla, Antigua and Barbuda, Dominica, Grenada, Montserrat and St. Kitts and Nevis. Dominica and St. Kitts assessments while finalized were yet to be peer reviewed.

${ }^{5}$ As defined in the IMF Good Practice Note (GPN) on 'Sequencing PFM Reform' (Diamond, 2013).
} 


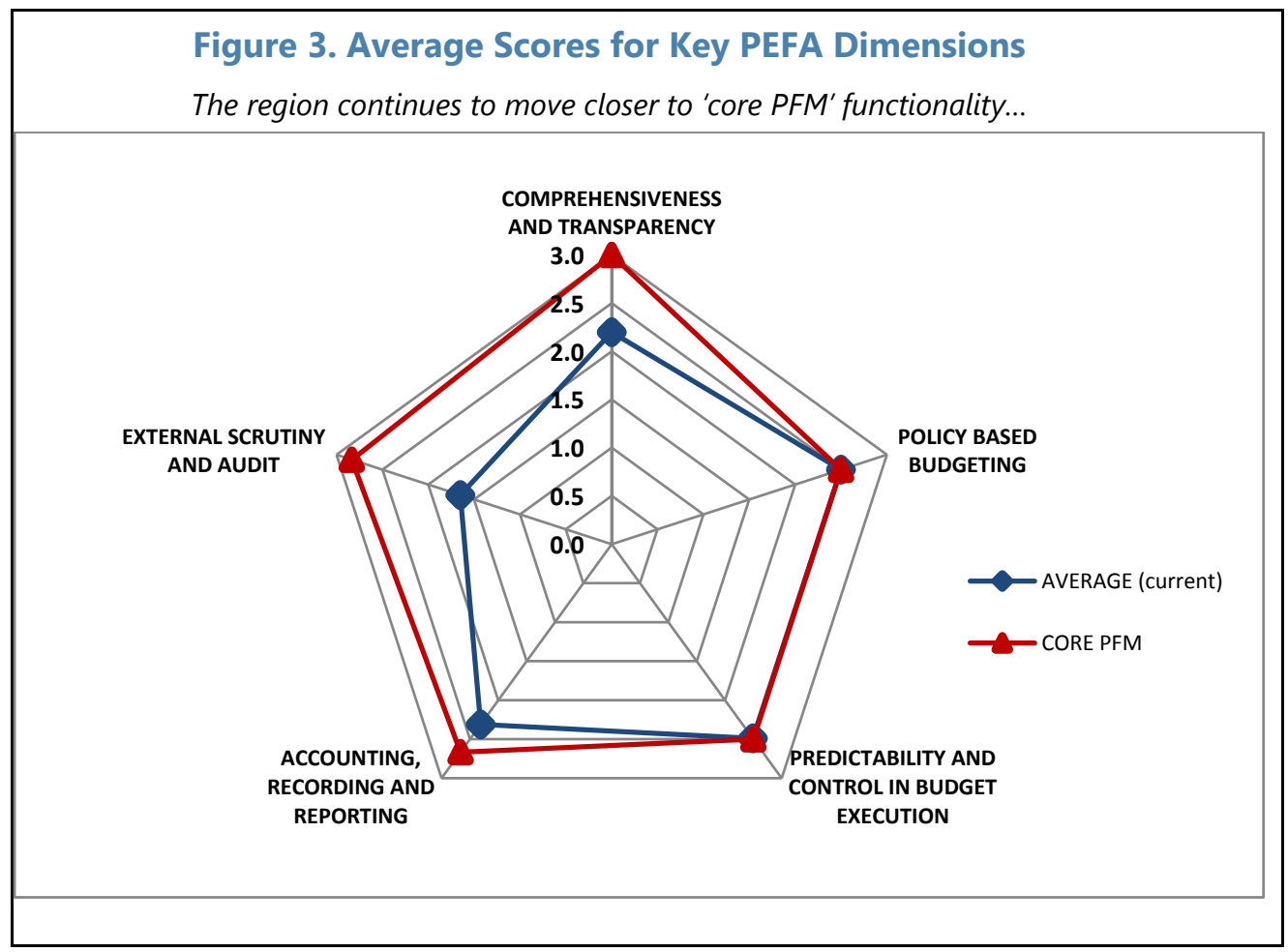

Implementation of a modern and consistent PFM legal framework across the ECCU is progressing. Antigua and Barbuda (implemented by IMF Headquarters with European Union funding), St. Lucia (under the Financial Management in the Caribbean program ${ }^{6}$ ) and Dominica have final draft legislation for legislative consideration, and Anguilla and St. Vincent and the Grenadines have initial drafts under review. Grenada passed revisions to its new PFM legislation and has adopted revised regulations (FMC).

Management of fiscal risks. Since the start of CARTAC's involvement in early 2014, Grenada has achieved significant traction in implementing reforms both at the sectoral level, and within individual State Owned Enterprises (SOE). The CARTAC framework has been successful in driving reforms at the enterprise level, which is starting to be reflected in improved operating performance. For example, with Gravel and Concrete the company (under new management) has turned its operations around, focused on cost reductions, and is now generating cash profits from operations. Within the Housing Authority, the accounts have been reorganized according to profit centers with accountability on each operating unit to generate cash profits. Specific targets have been established for sales that will allow the Authority to breakeven/earn a small profit.

Anguilla and St. Vincent and the Grenadines received diagnostic assessments and training in the implementation of the CARTAC SOE/SB ownership manual (bringing to six the number of countries implementing the manual). The six countries undergoing this reform were bought together in a

${ }^{6}$ FMC - Financial Management in the Caribbean program financed by Canada and executed by the Fiscal Affairs Department (FAD). 
small workshop to share experiences and better practices in implementing the CARTAC reform Grenada gave a compelling presentation on achievements to date and shared reporting templates and other material to aid the reform effort in the region.

Regional budget preparation reform has been strengthened, with new countries adopting the strategic budgeting reform agenda. St. Lucia's budgets were prepared according to the strategic budget reform methodology and presented in the revised program budgeting format, including non-financial performance information. St. Vincent and the Grenadines, together with Grenada have also used the new reform process, and piloted program budgeting reporting in their budget presentations for the first time, before moving to full implementation in 2017. Budget preparation reform was further sustained through the development of comprehensive budget manuals. New manuals were developed for St. Lucia and St. Vincent and the Grenadines; bringing to nine the number of CARTAC countries with comprehensive budget preparation manuals. The budget manuals include a gender budgeting perspective.

Compliance with International Public Sector Accounting Standards (IPSAS) has made great progress. The annual IPSAS cash basis workshop was repeated during the year to evaluate compliance with the new exposure draft. As a result, Anguilla, and St. Kitts and Nevis were assessed as fully compliant.

During FY16, St. Kitts and Nevis and St. Lucia were the recipients of diagnostics to assist the authorities to further develop their internal audit function for compliance with international standards.

The ECCU has been a major recipient of technical assistance in macroeconomic analysis and programming. Over the past year, CARTAC has responded to various unforeseen requests for assistance, including; assistance to the authorities in Dominica to assess the economic consequences of Tropical Storm Erika. In early January 2016, Anguilla requested assistance at short notice to assist with a medium-term fiscal strategy to meet requirements asked of them from United Kingdom's Foreign and Commonwealth Office.

The concerted effort to implement macroeconomic forecasting capabilities within ECCU Ministries of Finance led to improved capacities. Grenada, St. Lucia, and St Kitts and Nevis, as well as Anguilla, are all taking active roles in this vital function as a direct result of TA from CARTAC. In all these countries, CARTAC-designed frameworks are now being utilized by the authorities.

In FY16 the Macroeconomic program led a workshop for all ECCU members to produce a medium-term fiscal strategy consistent with the 60 percent debt to GDP target by 2030. All ECCU members submitted a formal report to their Cabinets following the workshop. The Macroeconomic and PFM programs jointly hosted a workshop in July 2015 to ensure that macroeconomic programming exercises are better integrated into the budget processes of participants. Outside of these workshops, technical officials from St. Lucia, Grenada and St. 
Kitts and Nevis have begun to submit their own fiscal strategies to Cabinet, as part of their regular duties.

CARTAC's TA in the area of financial stability revolves around: (i) stress-testing various aspects of the financial system; (ii) preparing financial soundness indicators (FSIs) for deposittaking institutions, and financial stability and health indicators (FSHIs) for the non-deposit taking segments of the financial sector; (iii) developing macro-prudential and systemic risk indicators (MPSIs and SRIs respectively) to bolster overall financial sector risk management and, in a few instances, (iv) assisting countries to refine their financial stability reports.

Work in the area of stress testing dominated CARTAC's financial stability work plan during FY16. Considerable progress was made with the conduct of an extensive dynamic modeling and stress-testing exercise of seven domestic and four international banks spread across the ECCU region (Antigua and Barbuda, Dominica, Grenada, St. Kitts and Nevis, Montserrat, St. Lucia, and St. Vincent and the Grenadines). The major focus of the project was to help the ECCB assess the financial situation of key banks in the ECCU region, as well as to enhance the capacity of staff to conduct regular stress tests of the ECCU banking system. This multi-country TA project served as an important complement to a larger project being undertaken by the IMF, the World Bank and the Caribbean Development Bank (CDB), focused on implementing an overall restructuring and resolution strategy for the ECCU banking system. In addition, specialized internal seminars on stress-testing and macro-prudential indicators were delivered for around 20 regulatory staff of the ECCB and for the Grenada Authority for the Regulation of Financial Institutions (GARFIN).

In the area of building financial resilience (by developing effective crisis management plans and resolution strategies), work focused inter alia on: (i) training GARFIN staff on crisis management and resolution frameworks, and (ii) improving the capacity of regulators in all segments of the financial system on crisis prevention, preparedness/management, and resolution frameworks. CARTAC conducted training on financial crisis management for the regulatory staff of GARFIN and in conjunction with the Central Bank of Barbados, hosted a three-day Conference on the theme "Building Resilience to Financial Crises in the Caribbean: The Role of Crisis Management Policies, Metrics and Plans" in March 2016. This Conference brought together 74 stakeholders from national regulatory authorities, the regional regulatory associations and key international bodies (including the IMF, World Bank and the FSB) to discuss how the Caribbean region could best approach the design of institutional and operational frameworks to withstand financial sector and economy-wide disruptions. ${ }^{7}$ Drawing on new information presented at this conference, some ECCU members (Grenada and

\footnotetext{
7 Some key areas covered in the Conference include (a) Key Components of a Well-Designed Crisis Management Plan (b) Legal and Institutional Foundations for Crisis Management (c) Resolving Systemically Important Cross-Border Institutions: Resolution Plans, Tools and Financing Options (d) Simulating Financial Crisis Management Plans and (e) Developing Appropriate Financial Safety Nets.
} 
St. Vincent and the Grenadines) have approached CARTAC for technical assistance in preparing strengthening their national financial sector crisis preparedness plans and resolution frameworks.

In the area of financial reporting, CARTAC also worked with the ECCB to train staff on financial stability reporting, and to review and strengthen the first draft of the ECCU financial stability report.

With regard to financial sector supervision, eighteen of the 20 CARTAC member countries are now fully on board to implement the standardized approach to Basel I/ in the Caribbean. During FY16, the ECCU adapted the revised Basel reporting forms, and the following Basel Guidelines: (i) credit risk; (ii) operational risks; (iii) market risks; (iv) interest rate risk in the banking book; (v) supervisory review process (pillar II), and disclosure and transparency (pillar III);

During FY16, several of the single regulatory authorities in the ECCU have also requested TA to implement Basel II for the off-shore banking sector. To this end, CARTAC has provided TA to Antigua and Barbuda, St. Vincent and the Grenadines, St. Lucia, and St. Kitts and Nevis to review their supervisory frameworks to ensure adequacy to implement Basel II. Antigua and Barbuda have already submitted the revised reporting forms and guidelines to their off-shore banking industry for comments and feedback.

Antigua and Barbuda, Dominica, Montserrat, St. Lucia, St. Vincent and the Grenadines, and St. Kitts and Nevis were the recipients of TA aimed at: (i) building capacity/training in risk-based supervision (RBS); (ii) documenting and formalizing their approach to risk-based supervision, and (iii) developing risk profiles for credit unions, insurance companies, pension funds and securities firms. RBS guidelines were reviewed to include: (i) the assessment of risk profiles in financial institutions; (ii) assessment of inherent risks such as credit, market, operational, strategic and reputational risks; and (iii) assessment of corporate governance oversight and governance functions and supervisory interventions as well as assessing the impact of risks on the firm's capital, profitability and liquidity. The RBS framework for Antigua and Barbuda is now published on the authority's website, and the authorities have incorporated the RBS framework in their onsite reviews of banks and non-banks as well as the offsite surveillance and monitoring of financial institutions.

Three regional workshops were held during FY16 for the non-bank sectors (insurance, pensions and securities). Eighty eight persons, representing 18 of 20 CARTAC member countries, participated in the insurance workshop, while the pensions workshop had 69 participants, representing 17 of the 20 CARTAC member countries. The insurance workshop focused on several key issues including cross border supervision, capital adequacy, solvency and stress testing, and conduct and disclosure; while the pension workshop focused on crisis 
management, risk based supervision, managing investments and offsite surveillance. The securities training workshop was held April 20 - 22 in The Bahamas with approximately 40 participants. The workshop focused on capital markets development, payment and settlement systems, risk-based supervision, and crisis preparedness. Follow-up on earlier training provided indicated that the authorities have incorporated training content into their on-site review and offsite monitoring of licensed financial institutions.

During the year, CARTAC in collaboration with the Caribbean Association of Credit Union Supervisors (CACS) held its regional credit union training workshop under the theme "Strengthening Credit Union Supervision and Regulation in the Caribbean." Regional regulators were trained on credit risk/loan review, risk weighted capital adequacy requirements, risk assessment and stress testing, supervisory intervention and corporate governance. Key among the challenges facing regulators as discussed during the workshop were: (i) inordinate delays in promulgating regulations for credit union supervision in the ECCU; (ii) challenges in passing legislation in some countries; (iii) transition from self-regulated organizations (SROs) such as credit union leagues, to an independent regulator such as the central bank, and (iv) poor credit quality evidenced by high levels of non-performing loans.

TA was also provided to:

- The first CARTAC regional financial sector strategic meeting focused on: (a) regional financial sector supervision and stability issues, challenges and TA responses; (b) financial sector reform; (c) macro prudential surveillance; (d) crisis management; (e) Basel II/III implementation, and $(f)$ risk-based supervision.

- The financial stability and financial sector supervision programs jointly hosted the financial strategic workshop with 59 participants drawn from the banking, insurance, pension, credit union, and capital market regulatory authorities in 17 CARTAC member countries. The objectives of the financial stability aspect of the workshop were to: (a) provide training in the areas of macro-prudential policy formulation as well as macroprudential indicators and systemic risk measures; (b) identify weaknesses in the financial stability frameworks of member states; and (c) to gauge the on-going impact of the newly-established financial stability program.

During 2014-15 the CARTAC program on economic and financial statistics concentrated on improving external sector, national accounts and prices statistics in ECCU countries. Good progress is being made by a number of countries in expanding the range and improving the quality of their annual national accounts. Improved annual GDP by expenditure (GDP-E) estimates have been compiled using the commodity flow approach by Grenada (to be released by December 2016) and St. Vincent and the Grenadines (released in May 2016); New methodologies to be used to compile GDP-E estimates for St. Kitts and Nevis, have been developed. Comprehensive reviews of the national accounts concepts, sources and methods 
have been completed for Antigua and Barbuda, St. Kitts and Nevis, St. Lucia, and St. Vincent and the Grenadines. Supply and use table (SUT) frameworks have been developed for Antigua and Barbuda, St. Kitts and Nevis, St. Lucia, and St. Vincent and the Grenadines.

Increased efforts are being made by countries to develop or improve quarterly national accounts estimates. The capacity of the Central Statistics Office in Dominica to compile quarterly GDP-P estimates has been strengthened; and methodologies to compile quarterly GDP-P have been developed for St. Kitts and Nevis, St. Lucia, and St. Vincent and the Grenadines.

Progress is also being made by countries to improve prices statistics and inflation management. The new reweighted consumer price index (CPI) series for Montserrat was released in September 2015, consistent with international standards. In addition to improvements in the compilation methodology, a new Federal CPI is being compiled for St. Kitts and Nevis.

CARTAC is continuing to coordinate its activities with other TA providers in the region, including the Project for the Regional Advancement of Statistics in the Caribbean (PRASC) ${ }^{8}$ being delivered by Statistics Canada on improving data sources and compiling national accounts and prices statistics; the assistance provided by the ECCB in reviewing the annual national accounts of its member states; and the consultants engaged by the Caribbean Community Secretariat during 2015 and 2016 to provide broad level advice on implementing the 2008 System of National Accounts (2008 SNA).

External sector statistics (ESS) focus on supporting the improvement of methodologies and compilation practices to better measure external transactions (balance of payments - BOP) and positions (international investment position - IIP), through the adoption of good practices and the latest internationally accepted guidelines and standards.

ECCU's balance of payments (BOP) data has been reviewed and IIP data produced for first time. Preliminary aggregated 2014 BOP and IIP statistics following the BPM6 methodology were produced and released by the ECCB in coordination with national statistics offices for the eight ECCU members and the ECCU. The ECCB plans to publish comprehensive balance of payments and IIP data by the end of 2016. The estimates benefited from CARTAC TA to develop and administer new surveys, and train data compilers and survey respondents. CARTAC continue

\footnotetext{
${ }^{8}$ The PRASC is a Canadian Government funded initiative being implemented by Statistics Canada over 2015-2022. The participating states include Antigua and Barbuda, the Bahamas, Barbados, Belize, Dominica, Grenada, Guyana, Jamaica, Montserrat, St. Kitts and Nevis, St. Lucia, St. Vincent and the Grenadines, Suriname, and Trinidad and Tobago.
} 
assisting ECCU members in reviewing the recent revisions and identify balance of payments components that need further improvement.

Visitor and student expenditure data has improved in Antigua and Barbuda, St. Lucia, Dominica, and St. Kitts and Nevis. Estimates of visitor expenditure in the balance of payments for Antigua and Barbuda and St. Lucia are now based on the current survey of departing passengers. To better account for the transactions of nonresident students, over 450 students were also surveyed in Antigua and Barbuda in collaboration with the former office of the IMF's Resident Representative for the ECCU region. Dominica collected data on tuition fees received from nonresident students-one of the main components of exports of travel services for the country-through the implementation of a specific ESS survey form of offshore universities. In May 2016, St. Kitts and Nevis conducted visitor expenditure surveys, collecting required data to compile enhanced estimates for visitor expenditure in the balance of payments. 